\title{
Diversidad Vegetal Espontánea en Agroecosistemas Hortícolas de La Plata y su relación con diferentes estilos de Agricultura: Importancia para la sustentabilidad
}

\author{
Autor \\ Lic. Susana Andrea Stupino
}

Tesis para optar al Grado Académico de Doctor en Ciencias Naturales

Director: Dr. Jorge L. Frangi

Codirector: Ing. Agr. Santiago J. Sarandón 
En memoria de mis abuelas Luisa y Josefa 
A mis hijos Juan Cruz y Micaela A mi esposo Guillermo 


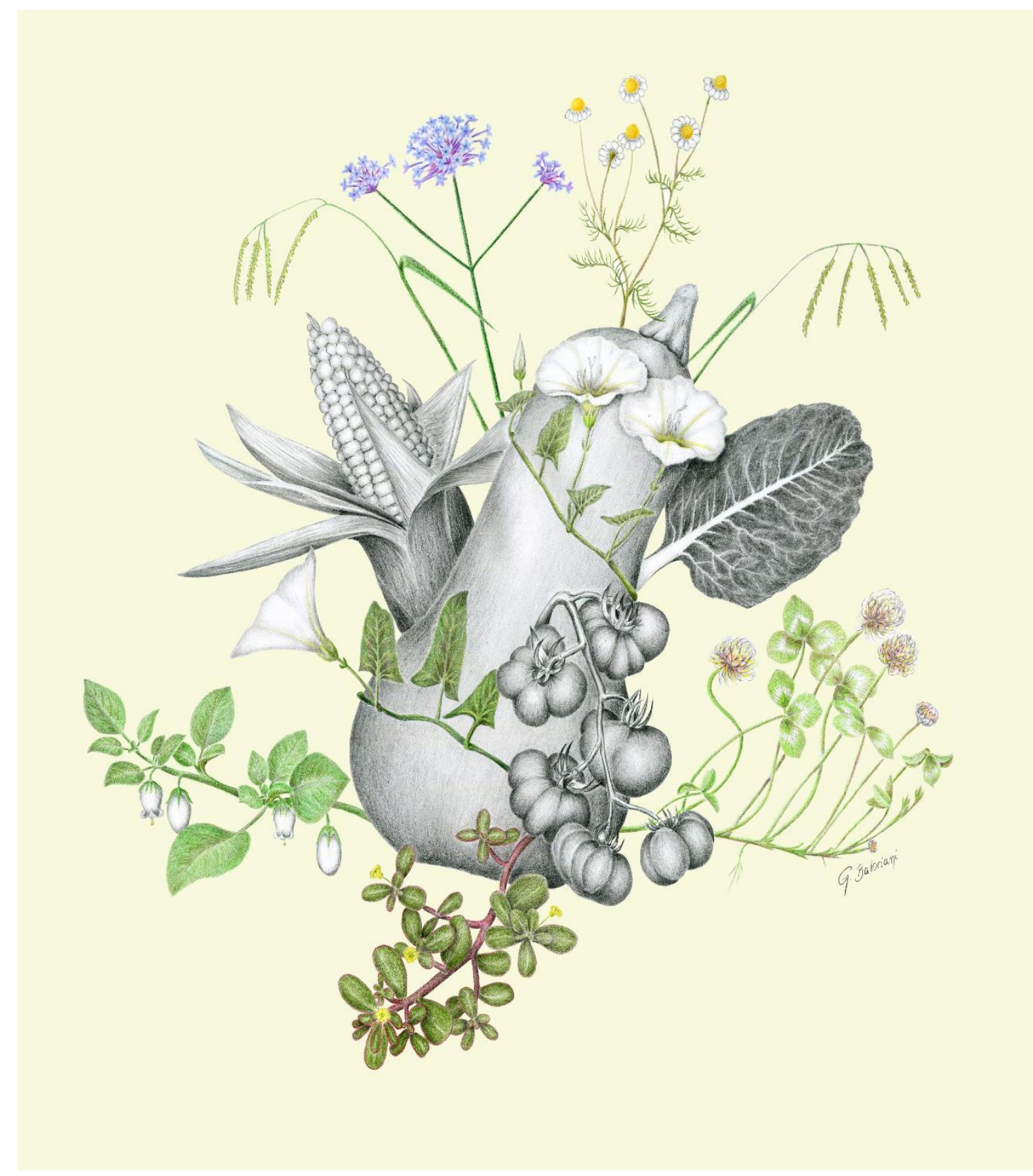

Composición artística de especies de plantas espontáneas asociadas a los cultivos hortícolas: realizada por el ilustrador científico Gabriel Baloriani con técnica mixta, lápices de colores policromos y lápices de grafito (septiembre de 2018). La imagen es una vista ampliada de la portada de la tesis.

Se destacan, en color y comenzando desde arriba y hacia la derecha, las especies Matricaria chamomilla (manzanilla), Paspalum dilatatum (pasto miel), Trifolium repens (trébol blanco), Portulaca oleracea (verdolaga), Salpichroa origanifolia (huevito de gallo) y Verbena bonariensis (verbena). En tonos grises se representan las siguientes especies de cultivo: en el centro Cucurbita mostacha (zapallo anco) y, comenzando desde abajo hacia la izquierda, Lycopersicon esculetum (tomate), Zea maiz (maíz) y Beta vulgaris var cicla (acelga). 


\section{AGRADECIMIENTOS}

Durante estos años de trabajo, son muchas las personas que han contribuido al avance y culminación de esta tesis, desde la formación académica, la amistad y el apoyo incondicional. Durante el camino recorrido he pasado por momentos laborales y personales felices y otros no tanto, donde las cosas de la vida parecían ponerse "cuesta arriba". Algunas veces me desanimé y otras veces aprendí a superarme. Por eso, esta tesis simboliza para mí más que un logro académico. Para aquellas lindas personas que me ayudaron a llegar a la meta va mi enorme agradecimiento y quiero mencionar especialmente:

A mi esposo Guillermo, porque su amor, su confianza en mí y su contención fueron fundamentales para finalizar la tesis. Por superar conmigo varias tormentas. Por acompañarme al campo y ocuparse de todo lo que implica la familia, especialmente en la etapa final de la tesis.

A mis queridos hijos Micaela y Juan Cruz, porque con sus abrazos, amor y alegría todo es más fácil.

A mi director Jorge Frangi, por haberme brindado un espacio de formación desde mi etapa como estudiante de la carrera de grado y por la confianza y el apoyo incondicional. Por el acompañamiento durante todos estos años y en el proceso de la tesis. Por trasmitirme su pasión por la Ecología y su amplia sabiduría, de esa que uno se queda admirado, y que además obliga a estar "atento al mensaje" para poder llevar a cabo ese pedido de un análisis nuevo, una idea, o cualquier tarea. Por creer en mí y apreciar mis logros. Por los consejos del trabajo y de la vida. Un maestro con mayúsculas.

A mi codirector Santiago Sarandón, por haber sido el generador de mi interés por la Agroecología desde las primeras charlas y haberme dado la posibilidad de ser docente en la materia. Por sus enseñanzas durante estos años y por trasmitirme su entusiasmo y empuje a hacer cosas nuevas. Por los aportes, reflexiones y consejos que fortalecieron este trabajo y mi formación profesional.

A los agricultores de las fincas visitadas, por su buena predisposición para realizar las entrevistas y trasmitirme sus conocimientos y experiencias. Por permitirme realizar el trabajo a "tranqueras abiertas". Un agradecimiento especial a Raúl y su familia y a Norma, con los que aprendí mucho de los cultivos y su manejo.

A todos los integrantes del LISEA, por el espacio de trabajo compartido durante estos años y con quienes fui creciendo en mi formación en la investigación, habiendo sido algunos de ellos docentes míos en materias y cursos de grado y posgrado. Especialmente quiero agradecer a Marcelo Arturi, por sus enseñanzas en el área de Ecología del Paisaje y por su colaboración en la elección de los métodos estadísticos y de muestreo, y por sus aportes en la interpretación de los resultados. Por su buena predisposición para ayudarme durante todo el período de trabajo. A Marcelo Barrera por sus aportes y ayuda en la elección e interpretación de los métodos de análisis de Ecología de Comunidades. A Mariana Dabadie por el tiempo compartido durante varios años, por las charlas de la vida y el cariño de siempre. A Carolina Pérez por incentivarme a finalizar esta etapa y sus cálidos consejos.

A mis compañeros de Agroecología, Margarita, Fernanda, Esteban, Mariana, Agustina Marilú, Claudia, María José y Carolina, de los cuales he aprendido que el trabajo en 
equipo e interdisciplinario siempre suma. Gracias por el apoyo, aprendizajes y por el tiempo compartido en estos años.

A Gabriel Baloriani, por la ilustración de la portada de la tesis y por su colaboración en el diseño.

Al personal del herbario del Museo de Ciencias Naturales (UNLP) por ayudarme en la corroboración de las especies identificadas.

A la Universidad Nacional de La Plata y al Consejo Nacional de Investigaciones Científicas y Técnicas (CONICET), instituciones que contribuyeron al desarrollo de parte de esta tesis durante las instancias como becaria.

A la Facultad de Ciencias Naturales y Museo por darme la posibilidad de aspirar al doctorado.

A mis padres Carlos y Estela, mis hermanos Silvia y Pablo, mis tíos, padrinos, primos y sobrinos, con quienes aprendo y comparto algo en común que identifica a la familia: la vida en el campo. A mis suegros Ester y Beto, por viajar expresamente a colaborar con la familia. A Gráfica Ressia por la impresión de la tesis.

A mis amigas-colegas Luciana, Ana Clara y Natalia, por la amistad que permanece desde los inicios de la carrera y por el apoyo, consejos y eternas charlas. Las quiero. Agradezco a Luciana por lo aprendido y compartido en instancia de becarias y por los consejos para organizar el manuscrito.

A mis amigas olavarrienses, Andrea, Silvina y Adriana, por acompañarme siempre y por los encuentros clásicos que son un cable a tierra. Gracias por estar.

A todos aquellos que, de diferente manera, me ayudaron a transitar este camino. Gracias. 


\section{RESUMEN}

La presente tesis tuvo como objetivo general caracterizar la composición y diversidad de plantas espontáneas, y la influencia que las distintas tipologías de manejo tienen sobre la misma en el Cinturón Hortícola de La Plata. Asimismo, se analiza la influencia del paisaje sobre la diversidad biológica en las fincas hortícolas locales.

Se relevaron las especies de plantas espontáneas y su cobertura en las parcelas de cultivo en 32 fincas hortícolas. Se entrevistó a los agricultores acerca de las prácticas de manejo que realizan y de la valoración personal que tienen sobre la vegetación espontánea. Se caracterizaron los tres tipos de manejo en la zona a partir de índices que describen el nivel de intensidad de uso de los insumos y recursos. Se calculó la riqueza y la diversidad, y se registraron los tipos funcionales de plantas, en distintas escalas espaciales: parcela de cultivo $(\alpha)$, entre parcelas diferentes $(\beta)$ y en el total del área cultivada o diversidad Gamma $(\gamma)$. A escala $\alpha$ se analizó la composición florística y su distribución en las parcelas de un mismo cultivo bajo diferente manejo. Se caracterizó la heterogeneidad del entorno del paisaje que rodea a las fincas a partir del relevamiento de unidades de hábitats cultivados y seminaturales. Se utilizaron diferentes métodos para establecer la relación entre la diversidad y las variables de manejo que influyen sobre las mismas.

El Cinturón Hortícola presentó una alta diversidad de especies, géneros, familias, tipos funcionales de plantas espontáneas y especies de valor utilitario. Entre los agricultores predominó la percepción negativa de la vegetación espontánea asociada al concepto de maleza y un escaso conocimiento de los valores de uso. Los beneficios reconocidos no son llevados a la práctica, teniendo mayor peso la valoración negativa, lo que influye en las decisiones de control. Esta percepción difirió entre los agricultores, siendo los orgánicos más tolerantes a la presencia de plantas.

Las fincas se distribuyeron conformando un gradiente de incremento de la intensidad de manejo que va de sistemas orgánicos a convencionales intensivos, con los convencionales de bajos insumos intermedios a los otros dos. Ese gradiente fue segmentado objetivamente en los tres tipos de manejo mediante un análisis multivariado que consideró un número significativo de variables socioeconómicas y de manejo de los cultivos. Las fincas orgánicas se caracterizaron por una mayor diversidad de cultivos en espacio y tiempo, sin uso de fertilizantes/herbicidas sintéticos, una mayor presencia de 
plantas espontáneas toleradas por el agricultor (umbral alto de malezas) y una tendencia a canales de comercialización diversificados. La mayor intensidad de manejo (propio de fincas convencionales intensivas) estuvo definida por el incremento el uso de herbicidas, fertilizantes sintéticos nitrogenados, el mayor uso del suelo, fincas de mayor superficie, ausencia de rotaciones y el bajo umbral de malezas (mayor intolerancia a las espontáneas). La modalidad de cultivo más intensiva correspondió al cultivo bajo cubierta (invernáculos). Aquellas fincas cuyos agricultores presentan similar situación socioeconómica, forma de producir, y lógica personal, se reconocen como un grupo dentro de las categorías establecidas. Sin embargo, otros factores como el acceso a los insumos, y al mercado influencian en las decisiones del agricultor.

Los sistemas orgánicos presentaron, respecto de los convencionales, una tendencia a una mayor riqueza, equitabilidad y diversidad en las distintas escalas de análisis $(\alpha, \beta, \gamma)$. Se destacaron por la presencia de nativas, perennes y especies raras o exclusivas. Esto se asoció con las características de sistemas menos disturbados.

A escala parcela de cultivo ( $\alpha$ ), el uso de fertilizantes, herbicidas y el bajo umbral de malezas (mayor intolerancia a la presencia de espontáneas) fueron las variables de manejo que tuvieron mayor efecto negativo sobre la diversidad Alfa. La diversidad Gamma y Beta tendieron a aumentar con la heterogeneidad de hábitats, lo que se explica por una mayor diversidad de cultivos y parcelas que incrementan la diversidad y recambio de especies entre parcelas cultivadas orgánicas. La heterogeneidad observada, se vincula con la forma de comercialización tendiente a canales de venta que requieren variedad de productos.

La complejidad del paisaje -dada por la proporción de hábitats seminaturales, cultivados y la diversidad de unidades espaciales-, no se relacionó con el incremento de la diversidad Gamma. La proporción de hábitats seminaturales y cultivados fue similar, sugiriendo un nivel intermedio de complejidad. Todos los tipos de manejo tuvieron entornos parecidos en cuanto al tipo, cobertura y riqueza de unidades del paisaje. Esto se explica porque las fincas orgánicas no se encuentran en una región particular como ocurre en otros países sino que están distribuidas inmersas en un paisaje diverso con predominio de áreas cultivadas convencionales.

En esta tesis se advirtió tanto la importancia de la heterogeneidad de hábitats, como el uso reducido de insumos (herbicidas y fertilizantes) y una valoración positiva de las plantas espontáneas como factores que determinan una mayor diversidad. La 
conservación de las especies podría lograrse a través de conversiones a sistemas agroecológicos, diversificados en su diseño. Para este proceso, es beneficioso aumentar la diversidad cultivada, el uso de prácticas alternativas a las químicas para manejar la vegetación espontánea, el uso eficiente de fertilizantes y la promoción de prácticas amigables con el medio ambiente. Las prácticas de cultivo, particularmente convencionales, tienden a empujar al ecosistema hacia nuevos estados. Por lo tanto, las opciones de gestión varían según el grado de cambio en el que se presentan los agroecosistemas y el reconocimiento de umbrales de intensidad de manejo que, de ser superados, es muy probable que comprometan la producción sustentable, o las respuestas sucesionales espontáneas y/o de manejo que ayuden a la rehabilitación o restauración del ecosistema.

Conservar la diversidad vegetal en los agroecosistemas sustentables debe ser un compromiso de los diferentes actores intervinientes en el manejo hortícola y en la gestión de políticas públicas relacionadas. Los resultados y conclusiones derivadas de esta tesis representan una contribución en este sentido. 


\section{SUMMARY}

The main objective of this thesis was to characterize the composition and diversity of spontaneous plants, and the influence that different types of management have on it in the Horticultural Belt of La Plata. Also, the influence of the landscape on the biological diversity in the local horticultural farms is analyzed.

The species of spontaneous plants and their coverage in cultivated plots located in 32 horticultural farms were surveyed. Farmers were interviewed about their management practices and their personal valuation of spontaneous vegetation. The three types of management in the area were characterized from indexes that describe the intensity level of inputs and resources use. The richness and diversity were calculated, and the functional types of plants were recorded, at different spatial scales: plot of crop ( $\alpha$ ), between different plots $(\beta)$ and in the total cultivated area or gamma diversity $(\gamma)$. At the $\alpha$ scale, the floristic composition and its distribution in the plots of the same crop under different management were analyzed. The heterogeneity of the landscape surrounding the farms was characterized by the survey of units of cultivated and semi-natural habitats. Different methods were used to establish the relationship between diversity and the management variables that influence them.

The Horticultural Belt presented a high diversity of species, genera, families, functional types of spontaneous plants and species of utilitarian value. Among the farmers, the negative perception of the spontaneous vegetation associated to the weed concept and a low knowledge of the values of use prevailed. The recognized benefits are not carried out in practice, with the negative aspects having a greater weight, which influences the control decisions. This perception differed among farmers, with organic producers being more tolerant of the presence of plants.

The farms were distributed along a gradient of increasing management intensity ranging from organic to intensive conventional systems, with conventional low-input farms intermediate to the other two. This gradient was objectively segmented in the three types of management by means of a multivariate analysis that considered a significant number of crop and socioeconomic management variables. The organic farms were characterized by a greater diversity of crops in space and time, without the use of synthetic fertilizers/herbicides, a greater presence of spontaneous plants tolerated by the farmer (high threshold of weeds) and a tendency to diversified marketing channels. The 
greater intensity of management (typical of intensive conventional farms) was defined by the increase in the use of herbicides, synthetic nitrogenous fertilizers, the greater use of land, farms with greater surface area, absence of crop rotations and a low threshold of weeds (greater intolerance to the spontaneous ones). The most intensive cultivation modality corresponded to the crop under cover (greenhouse). Those farms whose farmers have a similar socioeconomic situation, way of producing, and personal logic, are recognized as a group within the established categories. However, other factors such as access to inputs, and the market influence the decisions of the farmer.

The organic systems presented, with respect to the conventional ones, a tendency to greater richness, equitability and diversity in the different scales of analysis ( $\alpha, \beta, \gamma)$. They stood out for the presence of native, perennial and rare or exclusive species. This was associated with the characteristics of less disturbed systems.

At a plot scale ( $\alpha$ ), the use of fertilizers, herbicides and the low weed threshold (greater intolerance to the presence of spontaneous plants) were the management variables that had the greatest negative effect on Alpha diversity. Gamma and Beta diversity tended to increase with the heterogeneity of habitats, which is explained by a greater diversity of crops and plots that increase the diversity and exchange of species between organic cultivated plots. The observed heterogeneity is linked to the way of commercialization tending to sales channels that require a variety of products.

The complexity of the landscape, given by the proportion of semi-natural and cultivated habitats, and diversity of spatial units was not related to the increase of Gamma diversity. The proportion of semi-natural and cultivated habitats was similar, suggesting an intermediate level of complexity. All types of management had similar environments in terms of type, coverage and richness of landscape units. This is explained because organic farms are not found in a particular region as in other countries but are distributed immersed in a diverse landscape with a predominance of conventional cultivated areas.

In this thesis the importance of the heterogeneity of habitats, as well as the reduced use of inputs (herbicides and fertilizers) and a positive evaluation of spontaneous plants as factors that determine a greater diversity, were noticed. The conservation of the species could be achieved through conversions to agroecological systems, diversified in their design. For this process, it is beneficial to increase cultivated diversity, the employment of alternative (to chemical) practices to manage spontaneous vegetation, 
an efficient use of fertilizers, and the promotion of environmentally friendly practices. Cultivation practices, particularly conventional ones, tend to push the ecosystem towards new states. Therefore, management options vary according to the degree of change in which agroecosystems occur and the recognition of management intensity thresholds that, if exceeded, are likely to challenge sustainable production, or spontaneous successional responses and or of management that help the rehabilitation or restoration of the ecosystem.

Conserving plant diversity in sustainable agroecosystems should be a commitment of the different actors involved in the horticultural management and related public policies. The results and conclusions derived from this thesis represent a contribution in this regard. 
CAPÍTULO 1: IMPORTANCIA DEL TEMA, PRINCIPALES CONCEPTOS, ANTECEDENTES, OBJETIVOS E HIPÓTESIS

1. INTRODUCCIÓN 2

1.1 La importancia de la conservación de la biodiversidad en los agroecosistemas y su relación con la agricultura. 2

1.2 El origen de las plantas espontáneas y su importancia en la agricultura.............. 4

1.3 Escalas de análisis y factores determinantes de la biodiversidad.................... 7

1.4 Los estilos de agricultura convencionales y orgánicos. El rol del agricultor en la

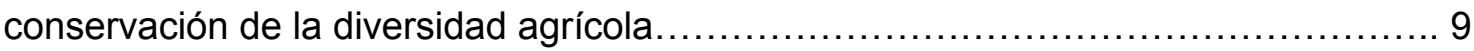

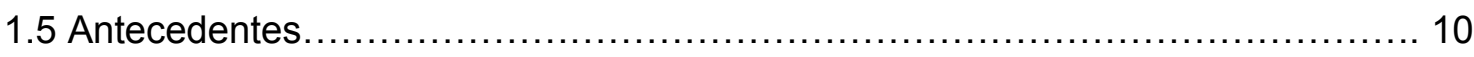

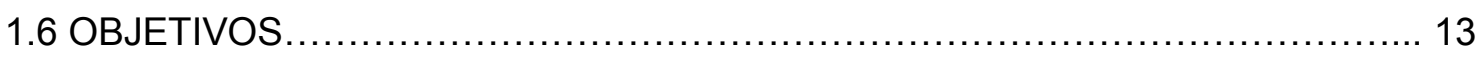

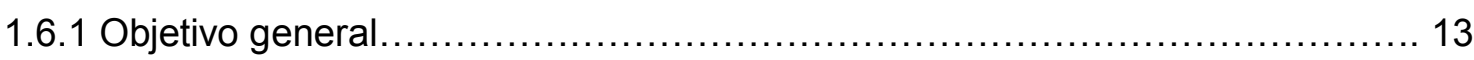

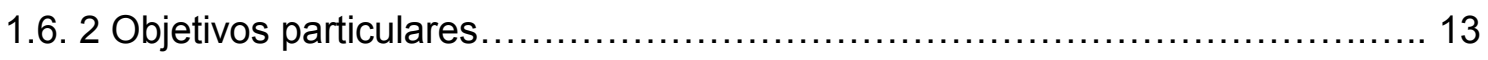

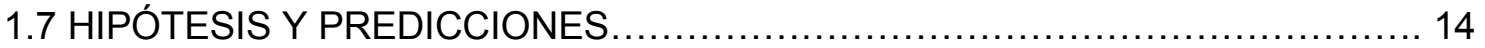

1.7.1 Caracterización de la biodiversidad vegetal en el área de estudio................. 14

1.7.2 Caracterización de los tipos de manejo del Cinturón Hortícola..................... 14

1.7.3 Influencia del manejo sobre la diversidad a escala parcela de cultivo $(\alpha) \ldots \ldots \ldots 14$

1.7.4 Influencia de la heterogeneidad de hábitats sobre la diversidad $\beta$ y $\gamma \ldots \ldots \ldots \ldots . . . .15$

1.7.5 Influencia del entorno a escala de finca................................................. 16

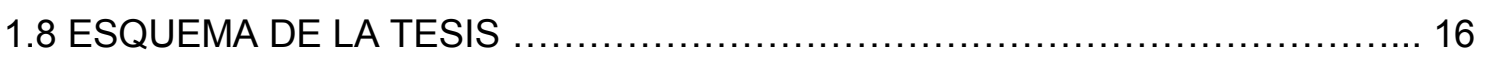

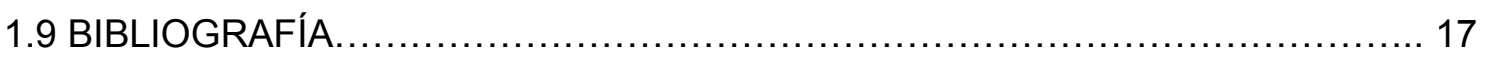

\section{CAPÍTULO 2: EL CINTURÓN HORTÍCOLA Y SU HISTORIA DE USO AGRÍCOLA}

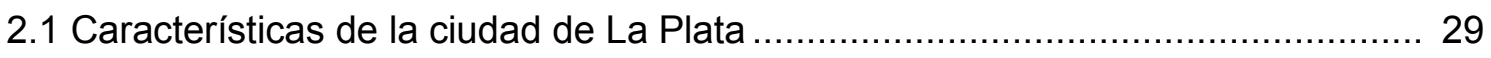

2.2 Conformación del cinturón y verde el origen de la horticultura........................ 30

2.3 Características de la región y las formas de manejo..................................33

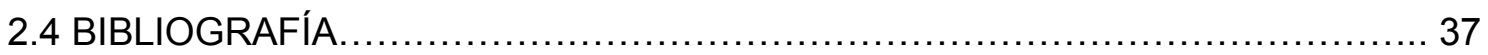

CAPÍTULO 3: CARACTERIZACIÓN DE LA DIVERSIDAD VEGETAL ESPONTÁNEA PRESENTE EN EL CINTURÓN HORTÍCOLA DE LA PLATA Y SU VALORACIÓN DESDE LA PERCEPCIÓN DE LOS AGRICULTORES

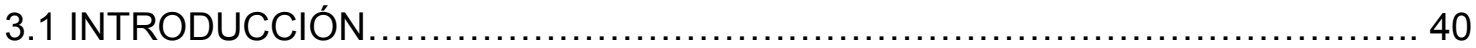

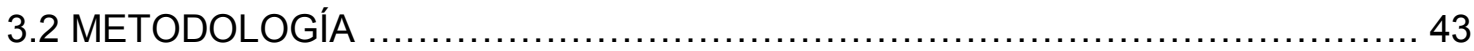

3.2.1 Selección de las fincas y realización de entrevistas................................4 43 
3.2.2 Muestreo de la vegetación espontánea................................... 44

3.2.3 Determinación de plantas y clasificación de grupos funcionales.................. 48

3.2.4 Evaluación de la percepción de los agricultores sobre las plantas espontáneas. 49

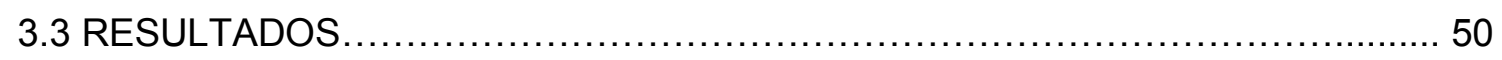

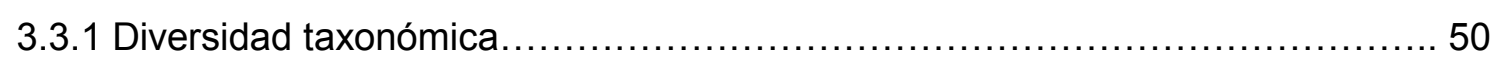

3.3.2 Importancia de las especies y estructura de grupos funcionales................. 55

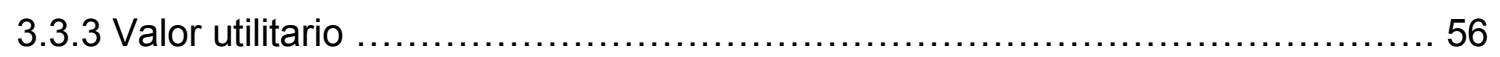

3.3.4 Percepción de los agricultores acerca de las espontáneas..................... 57

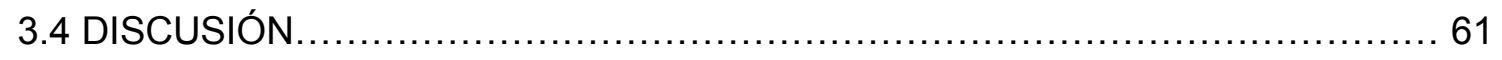

3.4.1 Caracterización de la biodiversidad presente en las fincas...................... 61

3.4.2 Percepción de los agricultores acerca de la vegetación espontánea..............63

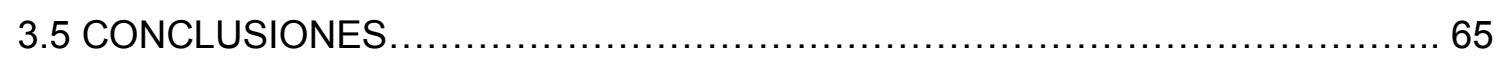

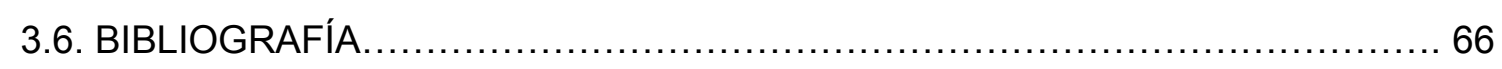

\section{CAPÍTULO 4: CARACTERIZACIÓN DE LOS TIPOS DE MANEJO DE LOS CULTIVOS} HORTÍCOLAS EN LOS ALREDEDORES DE LA PLATA

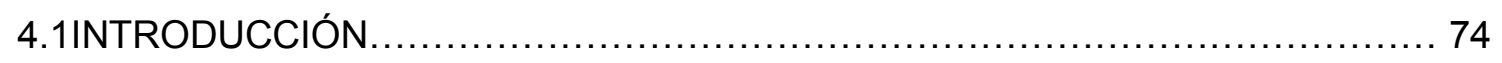

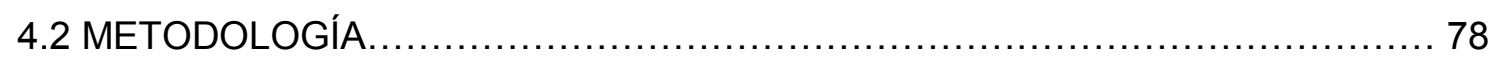

4.2.1 Selección de las fincas y realización de entrevistas............................ 78

4.2.2 Cuantificación de las variables relevadas.................................... 79

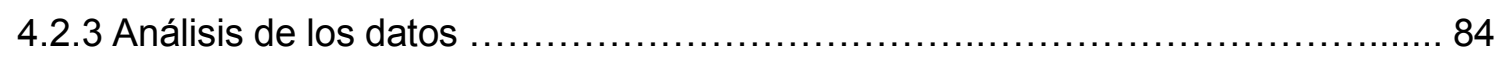

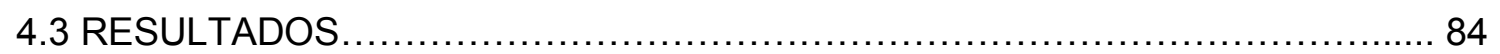

4.3.1 Características generales del manejo hortícola platense ........................ 84

4.3.2 Caracterización de las fincas a partir del método de ordenanción................. 89

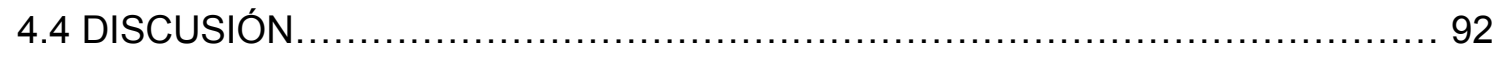

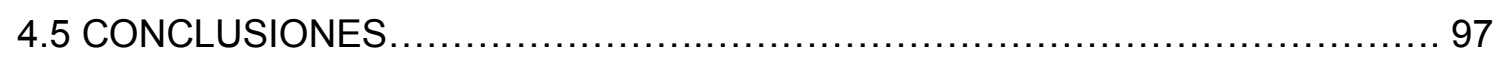

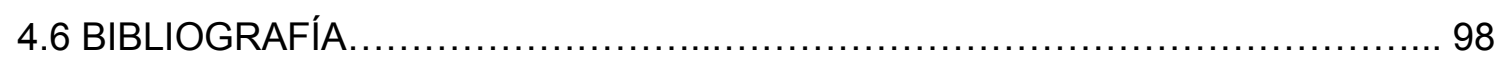

CAPÍTULO 5: ANALISIS DE LA DIVERSIDAD DE PLANTAS ESPONTÁNEAS EN PARCELAS CULTIVADAS BAJO DIFERENTE MANEJO. ESCALA ALFA

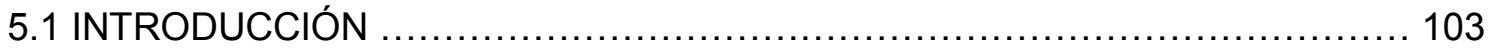

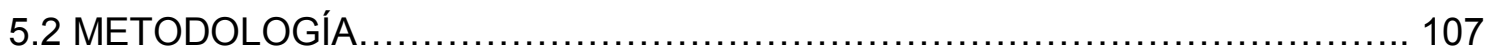

5.2 .1 Selección de las fincas................................................. 107

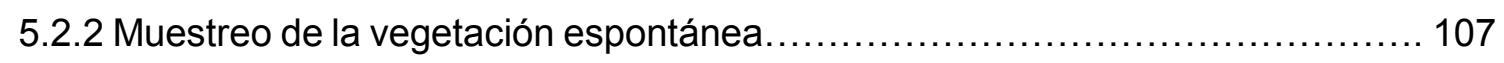




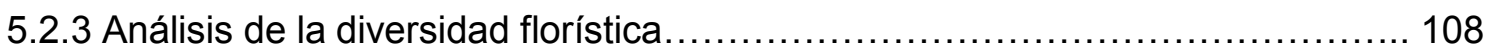

5.2.4 Análisis de la composición florística de los cultivos bajo diferente manejo...... 109

5.2.5 Análisis de la estructura de las tres categorías consideradas bajo el concepto

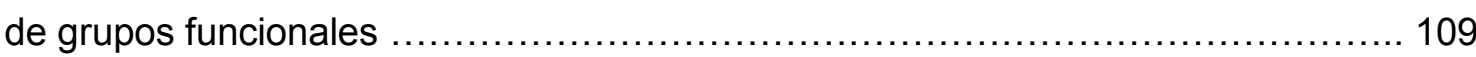

5.2.6 Diversidad Alfa en función de las prácticas de manejo........................ 110

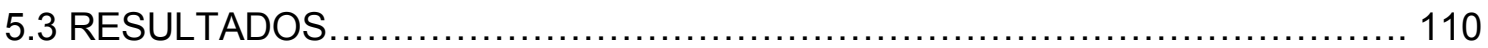

5.3.1 Diversidad y composición florística entre cultivos bajo diferente manejo......... 110

5.3.2 Análisis de la composición de especies según los grupos funcionales.......... 118

5.3.3 Diversidad Alfa en función de las prácticas de manejo......................... 121

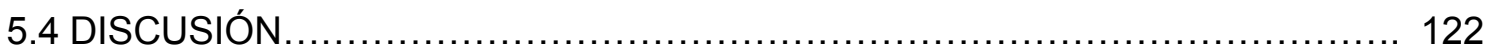

5.4.1 Diversidad dentro de las parcelas de cultivos bajo diferente manejo............ 122

5.4.2 Composición florística de los cultivos bajo diferente manejo................... 123

5.4.3 Composición de especies según los grupos funcionales ....................... 125

5.4.4 Diversidad Alfa en función de las prácticas de manejo ......................... 125

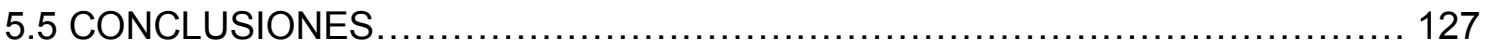

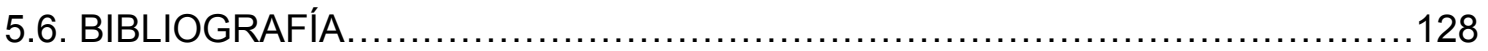

CAPÍTULO 6: ANÁLISIS DE LA RELACION ENTRE LA HETEROGENEIDAD DE HÁBITATS Y LA DIVERSIDAD DE PLANTAS ESPONTÁNEAS EN FINCAS BAJO DIFERENTE MANEJO. ESCALAS DE ANÁLISIS BETA, GAMMA Y PAISAJE.

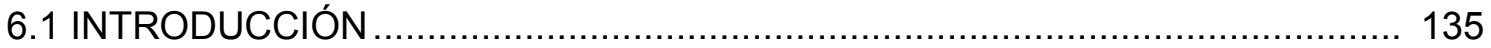

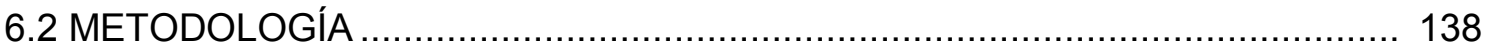

6.2.1 Relevamiento y cálculo de la diversidad Beta y Gamma a escala de finca...... 138

6.2.2 Análisis de correlación entre la diversidad vegetal y las variables que describen

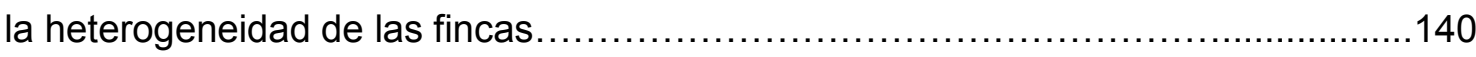

6.2.3 Localización de las fincas en el contexto del paisaje........................ 141

6.2.4 Definición del área de muestreo a nivel del paisaje.......................... 141

6.2.5 Clasificación y cuantificación de unidades del paisaje.......................... 142

6.2.6 Cálculo de índices de diversidad del paisaje................................... 143

6.2.7 Análisis de correlación entre la diversidad vegetal de las fincas y las variables que describen la heterogeneidad del paisaje ...................................... 144

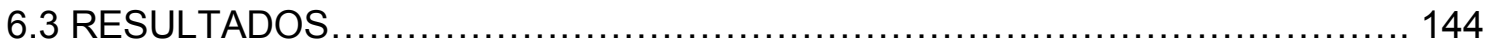

6.3.1 Relación entre la diversidad Beta y Gamma y la heterogeneidad de hábitats... 144

6.3.2 Caracterización del entorno del paisaje que rodea las fincas .................... 148

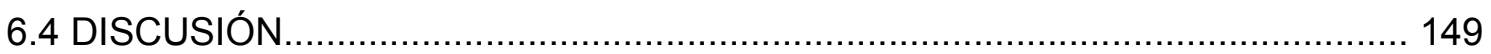

6.4.1 Relación entre la heterogeneidad y la diversidad Beta y Gamma............... 149 
6.4.2 Relación entre la diversidad y el entorno del paisaje ........................ 153

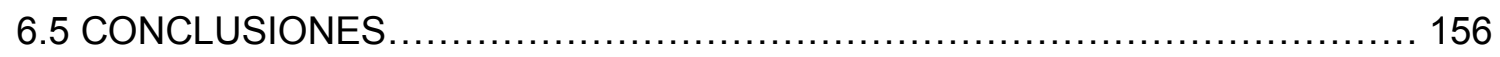

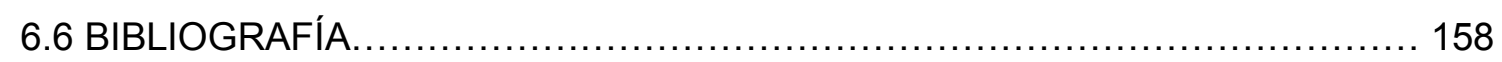

\section{CAPÍTULO 7: DISCUSIÓN GENERAL}

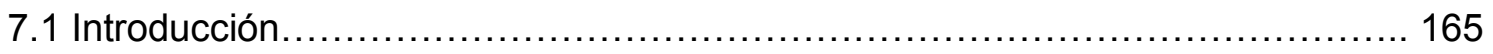

7.2 Biodiversidad presente en el Cinturón Hortícola Platense......................... 166

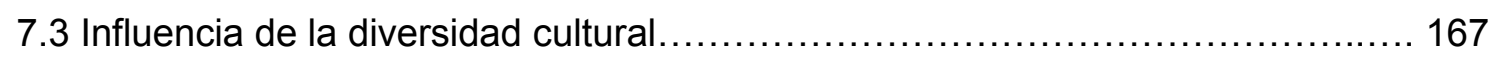

7.4 Los diferentes estilos de agricultura del Cinturón Hortícola....................... 168

7.5 Influencia del manejo a escala parcela de cultivo............................ 170

7.6 Escalas de diversidad Beta, Gamma y paisaje................................ 172

7.7 Posibilidades de conversión a sistemas de base agroecológica................... 175

7.8 Marco conceptual ecológico para comprender los cambios, estados y tendencia de los ecosistemas y factores que influyen sobre la diversidad en los ecosistemas hortícolas estudiados útiles para el diseño de sistemas sustentables................. 177

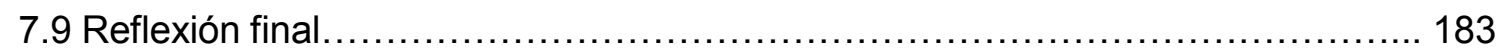

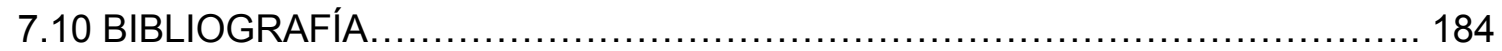




\section{CAPÍTULO 1}

IMPORTANCIA DEL TEMA, PRINCIPALES CONCEPTOS, ANTECEDENTES, OBJETIVOS E HIPÓTESIS

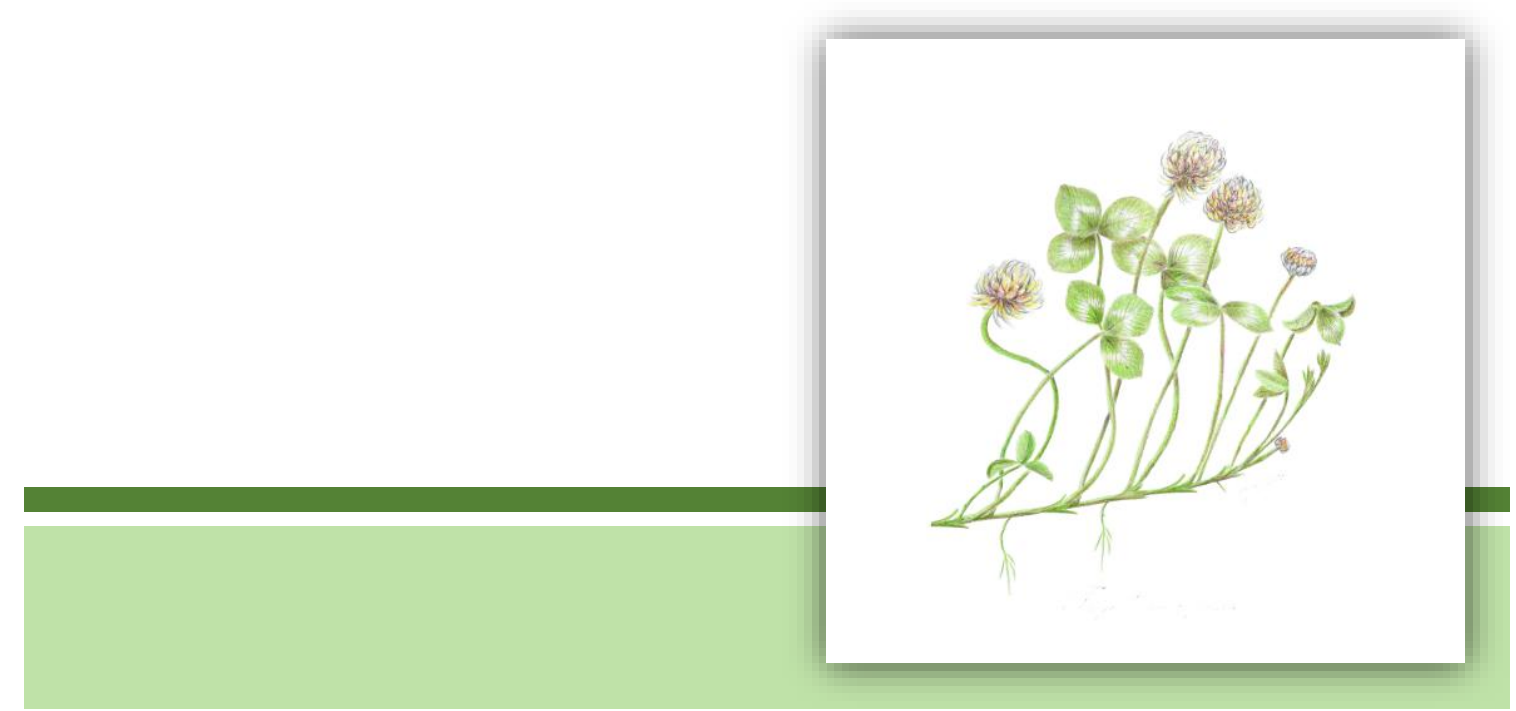




\section{CAPÍTULO 1}

Cualquier planta que insiste en crecer donde el agricultor quiere que crezca otra, es una maleza, pero según el buen criterio de la naturaleza está perfectamente en su lugar.

Edwin R. Spencer (1881-1964). Escritor, profesor de biología.

\section{IMPORTANCIA DEL TEMA, PRINCIPALES CONCEPTOS, ANTECEDENTES, OBJETIVOS E HIPÓTESIS}

\section{INTRODUCCIÓN}

\subsection{La importancia de la conservación de la biodiversidad en los agroecosistemas y su relación con la agricultura}

Unos de los desafíos actuales en la agricultura es lograr compatibilizar la producción con la conservación de los recursos naturales (UNEP, 1997; Tscharntke et al., 2005, 2012; Brussaard et al., 2010; Fisher et al., 2014). Las modificaciones ocurridas en los paisajes en las últimas décadas y la pérdida de especies, han llevado el problema de la conservación de la biodiversidad a tema de interés general (UNEP, 1997; Halffer et al., 1999; SCBD, 2008). Durante mucho tiempo, la preocupación por su conservación estuvo centrada en las áreas protegidas y otros ambientes naturales (Banks, 2004; Clergue et al., 2005; Fischer et al., 2014). Sin embargo, este compromiso no es ajeno al manejo de los agroecosistemas. Dado que la agricultura representa el uso de la tierra predominante en el mundo (ocupa alrededor del $40 \%$ de la superficie terrestre) y alberga una biodiversidad considerable (Pimentel et al., 1992; Halffer et al., 1999; Foley et al., 2005; FAO, 2013), es fundamental garantizar la conservación de la diversidad biológica en los sistemas agropecuarios (Pimentel et al., 1992; Matson et al., 1997; Banks, 2004; Clergue et al., 2005; Batáry et al., 2011).

La biodiversidad constituye la base de la vida en el planeta y de la sustentabilidad de los agroecosistemas. Proporciona los recursos genéticos y una variedad de servicios ecológicos que permiten reducir el uso de insumos externos en los sistemas productivos (Stupino et al., 2014; Garbach et al., 2014). Sin embargo, la agricultura es la principal actividad que afecta la biodiversidad (Altieri \& Nicholls, 1994; Banks, 2004; Kleijn et al., 2009; Jarvis et al., 2011).

Diversidad Vegetal Espontánea en Agroecosistemas Hortícolas de La Plata y su relación con diferentes estilos de Agricultura: Importancia 
La agricultura moderna deriva del modelo de la Revolución Verde, que surge a partir de 1960 con la idea de aumentar la producción de alimentos para satisfacer las demandas mundiales. Esto condujo, por un lado, a la expansión de la agricultura a través del reemplazo de ambientes naturales por agroecosistemas simplificados (Solbrig, 1999; Tscharntke et al., 2005; Gabriel et al., 2013). Otra tendencia impulsada por este modelo fue la intensificación productiva por unidad de superficie, es decir el aumento en el tiempo de utilización de la misma porción de tierra. Esto implica un incremento en el uso de los recursos y la aplicación de tecnologías como cultivares de alto rendimiento, fertilizantes y pesticidas químicos (Matson et al., 1997; Altieri \& Nicholls, 2000). Uno de los elementos clave de la intensificación es la simplificación y especialización en el proceso productivo con el objeto de lograr una mayor producción de alimentos y rentabilidad en el corto plazo (Gliessman, 2001; Swift et al., 2004). Esto involucra: 1) la reducción en el número de componentes planeados de la biodiversidad (cultivos, genes) (Matson et al., 1997; Vandermeer et al. ,1998; de la Fuente \& Suarez, 2008), 2) la reducción en la complejidad de labores o actividades realizadas por el agricultor a través de la mecanización, 3) el uso de insumos externos que reemplazan las interacciones bióticas y procesos internos, 4) la dependencia de la economía de mercado en la toma de decisiones, 5) la aparente reducción en la diversidad asociada a los cultivos (de la Fuente \& Suarez, 2008).

Una de las consecuencias de la intensificación agrícola ha sido la reducción de la heterogeneidad de hábitats (Benton et al., 2003; Foley et al., 2005; Tscharntke et al., 2005) y esto se ve reflejado en la baja diversidad que caracteriza a la agricultura actual, tanto a nivel finca como de paisaje (Altieri, 1999; Sarandón, 2002; SCDB, 2008). A escala de finca se observan pocas especies de cultivos, manejados a través de prácticas que favorecen la simplificación del agroecosistema (Evans, 1993; Matson et al., 1997; Gliessman, 2001; Benton et al., 2003; Swift et al., 2004). A su vez, la heterogeneidad en el paisaje depende del grado de diferenciación entre las fincas que lo integran. La economía de escala promueve la homogeneización de las mismas, a través de similares entradas de insumos y similares salidas de productos para el mercado. Esto determina agroecosistemas parecidos, con prácticas de manejo y controles de adversidades sincrónicos y estandarizados (Benton et al., 2003). Esta reducción de la diversidad entre fincas determina paisajes homogéneos (Altieri, 1999; Swift et al., 2004; Jarvis et al., 2011; FAO, 2013). 
Estos procesos señalados representan la principal causa de la disminución en la riqueza de especies de plantas y otros organismos e incluso la extinción de algunas especies espontáneas que dependen de los agroecosistemas (Wilson, 1993; Krebs et al., 1999; Sutcliffe \& Kay, 2000; van Elsen, 2000; Benton et al., 2003). Esta es una de las razones, junto con otros problemas ambientales, que han llevado a cuestionar ciertas prácticas agrícolas (Altieri, 1999; Tilman, 1999; Hole et al., 2005; Foley et al., 2005) y a la necesidad de promover sistemas tendientes a conservar la biodiversidad. Esto se logra bajo el paradigma de la agricultura sustentable, entendida como "aquella que mantiene en el tiempo un flujo de bienes y servicios que satisfagan las necesidades alimenticias, socioeconómicas y culturales de la población, dentro de los límites biofísicos que establece el correcto funcionamiento de los sistemas naturales (agroecosistemas) que lo soportan" (Sarandón et al., 2006; FAO, 2015). La tercera conferencia de las partes (COP 3 ) del convenio internacional sobre diversidad biológica, señala que la agricultura sustentable permite disminuir el impacto negativo sobre la diversidad biológica y unir los esfuerzos de conservación con los beneficios sociales y económicos (Sarandón, 2002). Además plantea la necesidad de evaluar el efecto de las prácticas agrícolas sobre los componentes de la biodiversidad y a promover prácticas alternativas y sistemas que garanticen su conservación (Altieri, 1999; Schmitzberger et al., 2005; Sarandón, 2002; Sarandón, 2009). Desde una perspectiva ecológica, la agricultura sustentable busca reducir el uso de insumos optimizando los procesos ecológicos que ocurren en el agroecosistema (Altieri \& Nicholls, 2000; Brussaard et al., 2010). Este objetivo se logra con el mantenimiento de la biodiversidad.

\subsection{El origen de las plantas espontáneas y su importancia en la agricultura}

De manera práctica, la diversidad biológica agrícola se puede subdividir en planeada y asociada. Los componentes de interés económico forman parte de la diversidad planeada, es decir, aquella que es incorporada al sistema por decisión del agricultor (Figura 1.1). La diversidad asociada comprende todos los organismos cuya presencia en el agroecosistema es mediada por la diversidad planeada (Vandermeer \& Perfecto, 1995; Swift et al., 2004; Jackson et al., 2007).

Diversidad Vegetal Espontánea en Agroecosistemas Hortícolas de La Plata y su relación con diferentes estilos de Agricultura: Importancia 


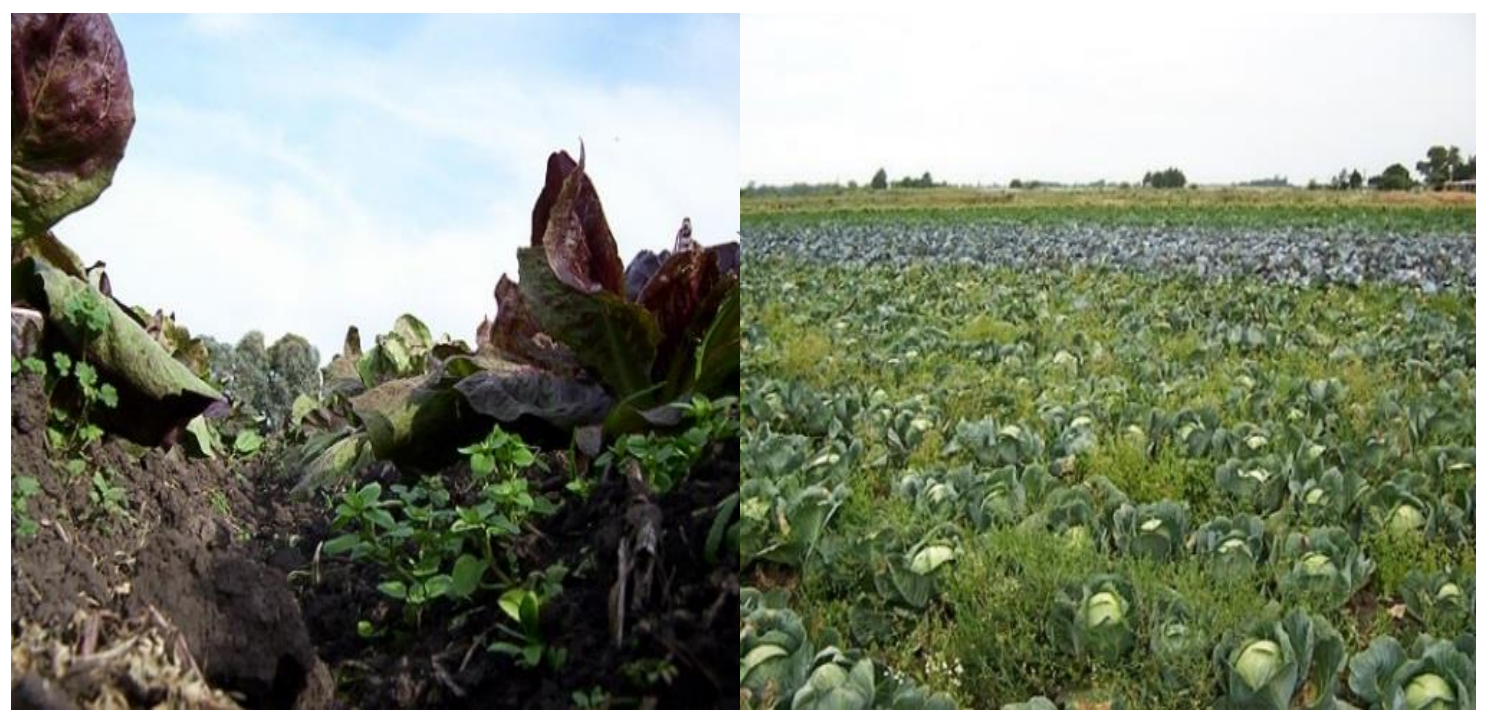

Figura 1.1. Componentes de la diversidad planeada (cultivo) y asociada (vegetación espontánea). Fotografía de la autora.

Las plantas que espontáneamente crecen junto a los cultivos forman parte de la diversidad asociada. Estas plantas reciben comúnmente el nombre de "malezas" o malas hierbas en países de habla castellana. Su origen etimológico proviene del latín malitia que significa malisia, expresión que denota el carácter perjudicial de las mismas (Pochettino, 2005). También se las denomina "yuyo“, que proviene del quechua "yuyu", hortaliza, término que en Argentina es utilizado con una acepción despectiva. Sin embargo, en otras regiones se refiere a las hierbas tiernas, comestibles 0 condimentarias (González et al., 2003). En este sentido, Rapoport et al. (2009) utiliza el término buenezas para las malezas comestibles, como paradoja que en algunos lugares se las utiliza como alimento y en otros lugares se las combate.

Existen varias definiciones de maleza, sin embargo, el concepto que prevalece está estrechamente vinculado a una consideración subjetiva y antropocéntrica que destaca el rechazo o daño que le genera al hombre (Radosevich et al., 2007). Comúnmente se define como maleza "una planta que crece fuera de lugar" (Radosevich et al., 2007)" o "plantas que llegan a ser perjudiciales o indeseables en determinado tiempo y lugar" (Marzocca, 1976).

El carácter perjudicial de las plantas espontáneas se refleja en dichos populares como "yerba mala nunca muere" o "sembrar cizaña". Esta última frase deriva de la parábola del trigo y la cizaña (planta silvestre similar al trigo y cuya harina es tóxica), “...Un hombre sembró buena semilla en su campo (trigo) y su enemigo en la noche sembró cizaña en medio del trigo..." (Mateo 13:24-30). Por otro lado, la presencia de 
plantas espontáneas en el campo muchas veces se asocia a haraganería, como en el proverbio "Pasé junto al campo del perezoso y junto a la viña del insensato, y todo eran cardos y ortigas que habían cubierto su haz (Marzocca, 1976)".

Tradicionalmente, las plantas espontáneas se han considerado negativas debido al impacto sobre los rendimientos de los cultivos (Altieri, 1997; Gliessman, 2001). Es por eso que, desde tiempos remotos hasta la actualidad, el control sobre estas plantas ha sido una práctica habitual en la que se han utilizado diferentes técnicas para su eliminación. Esta preocupación se ve reflejada en la relevancia que adquieren actualmente los herbicidas en la República Argentina, representando el $75 \%$ del volumen ( $\mathrm{Kg} / \mathrm{l})$ de productos fitosanitarios usados en la agricultura (CASAFE, 2012). No obstante, son pocas las especies que realmente causan severos problemas (Shepherd et al., 2003). De las 8000 especies vegetales consideradas malezas, solo 250 son consideradas problemáticas para la agricultura $(0,1 \%$ de la flora mundial) (Rodriguez Lagrega, 2006).

Por otro lado, las plantas asociadas a los cultivos pueden definirse según sus características biológicas. Según el rol ecológico, (en su sentido de especie ruderal) son especies adaptadas a hábitats disturbados o inestables, lo cual significa que son pioneras en las sucesiones secundarias (Harlan, 1992; Altieri, 1997; Grime, 1979). La agricultura constituye la mayor fuerza selectiva en la evolución de estas plantas espontáneas. El origen de las mismas puede ser múltiple: especies silvestres adaptadas a ambientes naturales disturbados que colonizan los agroecosistemas, especies cultivadas que escaparon o fueron abandonadas de la domesticación, híbridos de cultivos y plantas espontáneas, y coevolución con los cultivos a través de la selección y adaptación a disturbios recurrentes y disponibilidad de recursos concentrados (Harlan, 1976; di Castri \& Younes, 1996; Martinez-Ghersa et al., 2000). Por lo tanto, las especies espontáneas son un componente integral de los agroecosistemas e influencian la organización y funcionamiento de los mismos desde el origen de la agricultura. Cumplen una importante función en el agroecosistema que, muchas veces, es poco conocida o valorada (Marshall et al., 2002; 2003; Swift et al., 2004). Las especies espontáneas representan la base de la diversidad general, ya que constituyen la fuente de alimento y hábitat para organismos de otros niveles tróficos (Albrecht, 2003; Marshall et al., 2002; 2003). Proveen de hábitat a insectos benéficos que disminuyen las plagas en los cultivos (Altieri, 1988) y, de alimento a las aves granívoras e insectos polinizadores que las requieren como fuente de alimento directa (Marshall et al., 2003). Además, contribuyen a los diferentes servicios ecosistémicos de la biodiversidad como, el ciclado de 
nutrientes, la regulación del ciclo del agua, el control de plagas y enfermedades, la regulación del clima, la fotosíntesis y la detoxificación de químicos nocivos (Altieri \& Nicholls, 1999; Gliessman, 2001; Swift et al., 2004; de Bello et al., 2010; Garbach et al., 2014; Gaba et al., 2016). Las especies espontáneas constituyen la base de los recursos biológicos (genes) de las plantas agrícolas que son parte de la alimentación actual y que constituyen un potencial para el desarrollo en el futuro (Gliessman, 2001; SCBD, 2008). No solo se les atribuye un valor de uso para las actividades humanas (comestible, forrajero, ornamental o industrial), sino también se reconocen por sus diferentes representaciones en dialectos, usos imaginarios, simbólicos y medicinales en las poblaciones rurales (Gaba et al., 2016).

El mantenimiento de los servicios ecológicos y de la diversidad total del sistema está principalmente determinado por la naturaleza de las comunidades de plantas (Altieri \& Nicholls, 1999; Swift et al., 2004; Woodcock \& Pywell, 2010; Blake et al., 2011). Estos servicios permiten reducir el uso de insumos externos (Altieri \& Nicholls, 1994; Matson et al., 1997; Foley et al., 2005).

\subsection{Escalas de análisis y factores determinantes de la biodiversidad}

La diversidad puede ser analizada en distintos niveles de organización principales: genes, especies, y ecosistemas (di Castri \& Younes, 1996; Whittaker, 2001; Sarandón, 2009). Sin embargo, la mayoría de los análisis sobre su variación espacial refieren al número de especies que se encuentran en un área determinada (Rodriguez \& VasquezDominguez, 2003). Una forma de representar jerárquicamente la diversidad e incorporar la escala espacial, es a través de la caracterización en tres escalas: diversidad Alfa, Beta y Gamma (Whittaker, 1960; Magurran, 1988; Cornell \& Lawton, 1992; Whittaker et al., 2001; Pereyra y Moreno, 2013). En una escala local, Alfa ( $\alpha$ ) representa la diversidad dentro un de un hábitat o muestra (MacArthur, 1965; Whittaker, 1977; Magurran, 1988; Terradas, 2001). La diversidad Beta $(\beta)$, es la tasa de recambio de especies entre distintos hábitats y describe que tan diferente (o similar) es un rango de hábitats 0 muestras en términos de su composición específica. Por lo tanto, Alfa refiere a áreas homogéneas y Beta a la diferencia entre especies en áreas homogéneas que difieren en las condiciones ambientales (Sarr \& Huston, 2005; Tuomisto, 2010). La diversidad Gamma $(\gamma)$ es la riqueza de especies en un área determinada, incluyendo los hábitats que en ella se encuentran. Estos niveles definen aspectos diferentes de la diversidad y cuyos factores determinantes también difieren (Rodriguez \& Vasquez-Dominguez, 2003; Sarr \& Huston, 2005). La definición de las escalas varía según grupo de estudio y de los 
objetivos del trabajo (Shmida \& Wilson, 1985; Whittaker et al., 2001; Koleff \& Gaston, 2002).

El estudio de los factores determinantes de la diversidad de acuerdo con la escala espacial en la que intervienen, ha sido uno de los temas centrales de la ecología y de amplio debate (Hutchinson, 1953; Shmida \& Wilson, 1985; Hutson, 1994; Rosenzweig, 1995). El modo en que las especies se reúnen y coexisten en las comunidades depende de procesos de diversa duración temporal y distinto alcance espacial (Noss, 1990; Ricklefs \& Schluter, 1993; Terradas, 2001). Los factores determinantes actúan sobre el conjunto de especies a modo de filtros organizados jerárquicamente (Diamond, 1975). Este proceso de filtrado se concibe como la eliminación sucesiva de especies cuyos atributos funcionales no se ajustan a las condiciones ambientales impuestas por el filtro que opera a una determinada escala (Poggio, 2007). En la escala regional y de paisaje intervienen factores mayormente relacionados con la heterogeneidad y variabilidad ambiental como son las perturbaciones, patrones de uso del suelo y procesos de dispersión (Huston, 1994; Swift \& Anderson, 1994; Rosenzweig, 1995; Chase, 2003; Sarr et al., 2005). En una escala local, donde pueden situarse los sistemas modificados, los factores antrópicos son esenciales en la determinación de las comunidades de espontáneas (Andersson \& Milberg, 1998). El conjunto de especies espontáneas de una comunidad, en un ambiente agrícola en particular, puede acotarse a un sistema de producción o cultivo y es el resultado del filtrado de especies desde la flora regional. El filtrado se caracteriza por las limitaciones a la dispersión de las especies dentro de la región y, con mayor importancia a las restricciones del ambiente abiótico local como los disturbios, la disponibilidad de recursos y a las restricciones bióticas (Poggio, 2007, 2012).

La tierra es periódicamente disturbada a través de las distintas prácticas agrícolas orientadas a homogeneizar el ambiente para maximizar el rendimiento de los cultivos (Poggio, 2012). Dichas prácticas modifican los recursos disponibles (agua, fertilizantes), garantizan el establecimiento del cultivo (siembra) y controlan las adversidades. Este patrón cíclico, que es controlado por el manejo que realiza el agricultor, provee condiciones para el ensamblaje de las comunidades de plantas (Ghersa \& León 1999; Poggio, 2012; Petit et al., 2013). La fertilización, el método de control mecánico y/o químico y la rotación de cultivos son algunos de los factores que tiene influencia sobre la flora de espontáneas (Froud-Williams, 1987; Albrecht, 1995; Andersson \& Milber, 1998; Hÿvonen \& Salonen, 2002; Tang et al., 2013). Dado que el manejo representa un conjunto de estas variables que interactúan, se hace dificultoso poder evaluar el papel 
que desempeña cada factor en la determinación de la diversidad de especies. No obstante, resulta de interés poder establecer la importancia relativa de cada factor en sistemas bajo diferentes formas de manejo (Pysek \& Leps, 1991).

\subsection{Los estilos de agricultura convencionales y orgánicos. El rol del agricultor en la conservación de la diversidad agrícola}

Si bien se mencionaron anteriormente tendencias generales acerca del impacto de la agricultura moderna sobre la diversidad, es necesario señalar que existen diferentes estilos de agricultura, que pueden definirse, según van der Ploeg (1993), como distintas formas de "hacer" y "pensar". En los últimos años, la preocupación por las consecuencias ambientales de la agricultura moderna ha llevado al desarrollo de estilos de agricultura, que buscan compatibilizar niveles adecuados de producción y rentabilidad, con la conservación de los recursos, minimizando el impacto ambiental y buscando la calidad de los alimentos.

Los estilos de agricultura, que reúnen diferentes modalidades o tipos de manejo, pueden tener distinta influencia sobre la diversidad de especies espontáneas. De manera práctica, se pueden sintetizar en convencionales y orgánicos como los sistemas extremos. El término "convencional "es ampliamente aplicado a un rango de sistemas agrícolas modernos y su significado varía de acuerdo al objetivo de estudio (Hole et al., 2005). En ésta tesis se considera como convencional a aquel sistema hortícola caracterizado por el uso de productos químicos (fertilizantes, pesticidas y herbicidas) (Bengsston et al., 2005). Los sistemas de producción orgánicos o ecológicos están representados en la actualidad por un menor número de agricultores respecto de los convencionales. Estos sistemas existen en Argentina desde 1992, reconocidos oficialmente por el IASCAV (Resolución de la SAGyP 423/92). Las normas en que se basa son las del IFOAM (International Federation Organic Agriculture Movement, 2007). Según esta normativa "se entiende por orgánico, ecológico o biológico, en adelante orgánico, a todo sistema de producción sustentable en el tiempo que mediante el manejo racional de los recursos naturales, sin la utilización de productos de síntesis química, brinde alimentos sanos y abundantes, mantenga o incremente la fertilidad del suelo y la diversidad biológica y que, asimismo permita la identificación clara por parte de los consumidores, de las características señaladas a través de un sistema de certificación que las garantice". En estos sistemas se utilizan fertilizantes orgánicos (abonos), y se realiza el control de espontáneas de forma manual o mecánica, sin utilización de herbicidas.

Diversidad Vegetal Espontánea en Agroecosistemas Hortícolas de La Plata y su relación con diferentes estilos de Agricultura: Importancia 
Bajo similares condiciones de producción y en áreas comparables, las fincas no son necesariamente manejadas de la misma forma (Schmitzberger et al., 2005). En este sentido, la diversidad de espontáneas puede diferir a lo largo de los agroecosistemas, al estar asociadas a diferentes grados de disturbio que son generados por el tipo de labores realizadas (McLaughlin \& Mineau, 1995; Collins \& Qualset, 1999; de la Fuente et al., 1999; Suárez et al., 2001). Por ejemplo, el uso de herbicidas produce cambios en la composición de las comunidades de espontáneas (Ghersa \& Martínez-Ghersa, 2000; Marshall et al., 2002; Radosevich et al., 2007), disminuyendo la diversidad de especies (Moreby et al., 1994; Mc Laughlin \& Mineau, 1995), favoreciendo la presencia de especies anuales y monocotiledóneas (Mc Laughlin \& Mineau, 1995; Hyvonen et al., 2003; Shepherd et al., 2003). El uso de fertilizantes promueve el establecimiento de especies de mayor habilidad competitiva que se vuelven dominantes (Kleijn et al., 2016).

Dentro del efecto del manejo sobre la biodiversidad, el agricultor juega un papel fundamental, ya que decide qué organismos deben estar presentes (Swift, 2004) y cuáles deben controlarse (UNEP, 2000). El nivel de densidad tolerable o "umbral de daño" a partir del cual decide controlar la vegetación espontánea, está determinado por la percepción, conocimiento o creencias que el agricultor tenga acerca del rol que tienen las plantas espontáneas en su agroecosistema (funciones, posible daño) (Weibull et al., 2003; Roschewitz et al., 2005).

\subsection{Antecedentes}

El impacto de los estilos de agricultura sobre la biodiversidad vegetal ha sido analizado por diversos autores (McLaughlin \& Mineau, 1995; Swift et al., 2004; Schmitzberger et al., 2005). Cuando se comparan sistemas orgánicos y convencionales extensivos (monocultivos de cereales, por ejemplo), la tendencia encontrada es que la diversidad de especies es mayor en los sistemas bajo manejo orgánico (Krebs et al., 1999; van Elsen, 2000; Hyvonen et al., 2003; Roschewitz et al., 2005; Gabriel et al., 2010; Edesi et al., 2012; Henckel et al., 2015). Esto coincide con lo señalado en la revisión de Hole et al. (2005) y el meta-análisis de Bengtsson et al. (2005), Rahmann (2011), Winqvist et al. (2012) y Tuck et al. (2014). La riqueza de especies puede representar hasta un $30 \%$ más que en fincas convencionales y hasta un $70 \%$ en la escala de lote (Bengtsson et.al.; 2005; Bavec \& Bavec, 2015). Excepcionalmente se encontró una similar diversidad en ambas formas de manejo (Weibull et al., 2003).

Diversidad Vegetal Espontánea en Agroecosistemas Hortícolas de La Plata y su relación con diferentes estilos de Agricultura: Importancia 
La mayor diversidad encontrada en los sistemas orgánicos se asocia a un mayor número de especies raras o en peligro de extinción (Rydberg \& Milberg, 2000; Hole et.al., 2005; Roschewitz et al., 2005) especies perennes, nativas (van Elsen, 2000, Stupino et al., 2008) y un mayor número de dicotiledóneas (Moreby et al., 1994; Roschewitz et al., 2005; Armengot et al., 2012). Sin embargo, no se conoce bien cuáles son los atributos del manejo que permiten el mantenimiento de la biodiversidad (Henckel et al., 2015). Hole et al. (2005) señalan que los beneficios de la forma de producción orgánica estarían dados por una mayor calidad/cantidad de hábitats cultivados y no cultivados (parches) respecto de los convencionales, el uso reducido de insumos químicos y el manejo amigable ("sympathetic") de la vegetación espontánea dentro del área cultivada y los márgenes (Shepherd et al., 2003; Hole et al., 2005). Sin embargo, estas son opciones de manejo intrínsecas pero no exclusivas de la agricultura orgánica. Aunque la completa erradicación de espontáneas no es el objetivo de este estilo de agricultura (Lampkin, 2001), las adopciones de esta práctica por razones económicas, podría llevar a mantener una flora similar a la de algunos sistemas convencionales (Brooks et al., 1995; van Elsen, 2000). Krebs et al. (1999) señalan que los beneficios probablemente se relacionen a la heterogeneidad de hábitats y al bajo uso de insumos más que a cierta prescripción específica de la agricultura orgánica. A su vez, Benton et al. (2003) plantean que dicha heterogeneidad podría ser más importante que el no uso de químicos "per se". La variedad de cultivos determina una diversidad estructural y de hábitats que podría promover el recambio de especies entre cultivos (Beta) generando una mayor diversidad de espontáneas a nivel finca (Gamma) (Unwin et al., 1995; Palmer \& Maurer, 1997).

Por otro lado, la diversidad local de especies espontáneas puede estar determinada, en parte, por el paisaje circundante (Forman, 1995; Weibull \& Ostman, 2003; Roschewitz et al., 2005; Tscharntke et al., 2005; Henckel et al, 2015). Las especies acompañantes de los cultivos se adaptan a los ambientes disturbados a través de estrategias como la alta tasa reproductiva y de crecimiento. Sin embargo, la ocupación de un sitio disturbado puede depender en parte de propágulos que provienen de otros sitios circundantes (Terradas, 2001; Weibull \& Ostman, 2003; Roschewitz et al., 2005). En este sentido, la estructura y heterogeneidad del paisaje también contribuyen a la biodiversidad en el mosaico agrícola (Forman, 1995; Krebs et al., 1999; Burel \& Baudry, 2001; Benton et al., 2003; Baessler \& Klotz, 2006). Los paisajes más complejos o heterogéneos pueden contener un mayor número de especies que los paisajes simples, al presentar una mayor diversidad de hábitats naturales y cultivados 
alternativos que pueden actuar como fuente de propágulos para las fincas (Benton et al., 2003). Por lo tanto, la complejidad del paisaje puede mitigar la eliminación local de espontáneas a través del aporte de propágulos desde las fuentes externas de dispersión (Roschewitz et al., 2005; Tscharntke et al., 2005).

Pocos estudios, realizados en otras regiones, han evaluado la relación entre la riqueza local de especies espontáneas, el manejo y el contexto del paisaje (Balezentiene, 2011; Petit et al., 2013, 2016; Henckel et al., 2015). Algunos encontraron un efecto menor del contexto del paisaje en la composición y riqueza de especies (Weibul et al. 2003; Krauss et al., 2003). Roschewitz et al. (2005) en cambio, encontraron que tanto el manejo como la complejidad del paisaje (superficie de tierra cultivada) tienen influencia en la diversidad Gamma de espontáneas (Winqvist et al., 2012). La diversidad fue mayor en los sistemas orgánicos que en los convencionales y especialmente en paisajes simples con una alta proporción de áreas cultivadas (Bátary et al., 2011). En paisajes complejos ambos sistemas alcanzaron un nivel de diversidad similar, por lo que se concluye que el efecto de la complejidad del paisaje es mayor en los sistemas convencionales. Por lo tanto, si la heterogeneidad de hábitats a escala de finca favorece la presencia local de especies, el efecto de la complejidad del paisaje debería ser mayor en los sistemas hortícolas convencionales de baja heterogeneidad de hábitats (pocas especies cultivadas, grandes parcelas) y alto uso de insumos.

En el país, se ha evaluado la diversidad de las especies acompañantes de los cultivos para sistemas extensivos en distintas escalas espaciales (de la Fuente et al., 1999; Suárez et al., 2001; Poggio, 2005, 2007; Puricelli et al. 2012; Poggio et al., 2013). Sin embargo, para el área de estudio existen pocos antecedentes en los que se haya analizado la composición y diversidad de plantas espontáneas en sistemas hortícolas (Stupino et al., 2007, 2008; Paleologos et al., 2008; Marasas et al., 2011; Fernandez \& Marasas, 2015). Dado que los agroecosistemas pueden albergar una diversidad considerable, es esperable que el Cinturón Hortícola de La Plata presente una alta diversidad vegetal (Stupino et al., 2007; 2008). Por otro lado, los estudios en otras regiones se han realizado principalmente comparando sistemas de estructura similar, pero pocos han considerado sistemas convencionales altamente intensivos (Shepherd et al., 2003) o bien, no incluyen situaciones intermedias dentro de los estilos más contrastantes (Gardner \& Brown, 1998). Además pocos se refieren a sistemas hortícolas (Shepherd et al., 2003). La mayoría de los estudios diferencian de manera teórica los estilos sólo por el uso o no de insumos químicos sin tener en cuenta otras variables de manejo medibles que podrían tener impacto sobre la diversidad. Una de las cuestiones 
en éste sentido consiste en caracterizar el espectro o variantes de sistemas de manejo hortícola, y no sólo aquellos extremos estereotípicos contrastantes (orgánico y convencional), con los cuales se asocian los cambios en la composición y diversidad.

El impacto que los diferentes sistemas de producción mencionados tienen sobre la agrobiodiversidad debe así analizarse a través de su contribución al logro de una agricultura sustentable. En este sentido, la biodiversidad de estos sistemas puede ser esencial para permitir un manejo basado en un menor uso de insumos. Como los antecedentes lo señalan, no se conoce realmente qué aspectos del manejo son los que benefician el mantenimiento de una flora más diversa. Esto motiva el interés por conocer los estilos de agricultura que existen en el área de estudio y, los factores de manejo que afectan a la diversidad de especies espontáneas. La evaluación de los efectos que las diferentes prácticas agrícolas tienen sobre la agrobiodiversidad es un conocimiento imprescindible para alentar prácticas agrícolas más sostenibles y elaborar estrategias de extensión adecuada hacia los agricultores. Por otro lado, poco se conoce acerca de la composición y diversidad de plantas espontáneas en sistemas hortícolas. La caracterización de la flora a nivel zonal, permite conocer más acerca de la importancia de las especies y de su rol funcional asociado con los distintos componentes de la biodiversidad de la zona, para garantizan el mantenimiento de los servicios ecológicos que permiten el correcto funcionamiento del sistema en el marco indicado.

\subsection{OBJETIVOS}

\subsubsection{Objetivo General}

El objetivo general de esta tesis es caracterizar la diversidad y composición vegetal espontánea y analizar la influencia que las distintas modalidades de manejo tienen sobre la diversidad en la zona hortícola de La Plata. Asimismo se pretende reconocer la influencia del paisaje sobre la diversidad biológica en los distintos sistemas de horticultura empleados localmente.

\subsubsection{Objetivos particulares}

- Realizar un relevamiento y caracterización de diversidad y composición vegetal presente en el Cinturón Hortícola Platense. 
- Realizar un relevamiento y caracterización de las modalidades (combinación particular de prácticas) que tiene el manejo para la producción hortícola y de la vegetación espontánea asociada a los cultivos en las fincas.

- Identificar las principales variables de manejo que inciden sobre la diversidad, composición florística, y otras clasificaciones de plantas acompañantes de los cultivos a diferentes escalas.

- Caracterizar el paisaje que rodea a cada finca y evaluar si existe alguna contribución diferencial de los distintos paisajes a la biodiversidad de especies acompañantes de los cultivos de los distintos sistemas hortícolas.

\subsection{HIPÓTESIS Y PREDICCIONES}

\subsubsection{Caracterización de la biodiversidad vegetal en el área de estudio}

La heterogeneidad de hábitats afecta la diversidad de plantas espontáneas. En el Cinturón Hortícola Platense, debido principalmente a su complejidad de cultivos y manejos, ésto se manifiesta en una significativa diversidad de especies, géneros $y$ familias de espontáneas, grupos funcionales y especies de valor utilitario.

Independientemente del grado conocimiento que tenga el agricultor acerca de las especies acompañantes prevalece su percepción como malezas, reflejado en una dominante valoración de los aspectos negativos.

\subsubsection{Caracterización de los tipos de manejo del Cinturón Hortícola}

En la zona estudiada, las fincas suelen ser encuadradas en dos categorías principales de manejo: orgánico y convencional. No obstante, las diferentes lógicas de producción y de prácticas empleadas por los agricultores son dependientes de múltiples factores, lo que permite esperar que las fincas integren un gradiente de intensidad de manejo.

Dada la heterogeneidad que presenta la horticultura platense, es esperable que además del uso de productos químicos, existan otras variables que puedan resultar relevantes para caracterizar el manejo de la zona.

\subsubsection{Influencia del manejo sobre la diversidad a escala parcela de cultivo ( $\alpha)$}

Diversidad Vegetal Espontánea en Agroecosistemas Hortícolas de La Plata y su relación con diferentes estilos de Agricultura: Importancia 
Los componentes de la diversidad de las parcelas de tres cultivos (tomate, lechuga y maíz), conducidos a campo, dan resultados distintos de acuerdo con la diferencia en la intensidad del manejo (orgánico y convencional intensivo).

- Los cultivos orgánicos debido a su manejo menos intensivo, presentan una mayor riqueza, equitabilidad y diversidad que el que expresan los mismos cultivos bajo manejo convencional intensivo.

Las diferentes intensidades de manejo afectan de distinta manera la composición florística de especies acompañantes de los cultivos.

- Las parcelas con similar manejo tienen mayor semejanza florística entre sí que las parcelas de diferente manejo.

- Las parcelas convencionales son más parecidas entre sí en la composición de especies que las parcelas orgánicas.

La instalación de cultivos de ciclo corto implica conducir agroecosistemas que tienen características semejantes a aquellas de las etapas tempranas de la sucesión. La intensidad y duración de las técnicas aplicadas para causar o remover tensiones (stress) en cada tipo de manejo, afectan la composición estructural de tipos funcionales de plantas espontáneas.

La diversidad de plantas acompañantes se vincula con un complejo de variables, entre las que se hallan algunas asociadas con cada tipo de manejo, la historia de uso de la tierra, y la tolerancia del agricultor hacia las "malezas".

- La riqueza y diversidad de especies espontáneas disminuirán, principalmente con el aumento de uso de herbicidas y el menor umbral de daño considerado por el agricultor.

\subsubsection{Influencia de la heterogeneidad de hábitats sobre la diversidad $\beta$ y $\gamma$}

La heterogeneidad de hábitats favorece el aumento de la diversidad Beta y Gamma.

Diversidad Vegetal Espontánea en Agroecosistemas Hortícolas de La Plata y su relación con diferentes estilos de Agricultura: Importancia 
- El aumento del número de especies de cultivo y parcelas se corresponde con un aumento de la diversidad Beta (entre cultivos) y Gamma (riqueza total del área cultivada) de especies espontáneas.

- Esta mayor diversidad se traduce en un mayor número de especies espontáneas exclusivas, un mayor número de especies nativas, perennes y dicotiledóneas; esta diversidad es máxima en los cultivos orgánicos y disminuye hacia los cultivos convencionales intensivos.

\subsubsection{Influencia del entorno a escala de finca}

La complejidad del paisaje influencia la diversidad Gamma a escala de finca y su efecto varía según las particularidades de manejo.

- Existe correlación positiva entre el aumento de la complejidad del paisaje y la diversidad de especies espontáneas asociadas a los cultivos. Este efecto es mayor en las fincas convencionales de baja heterogeneidad de hábitats (pocos cultivos y parcelas).

\subsection{ESQUEMA DE LA TESIS}

El Capítulo 1 o introducción general provee al lector de un marco teórico relacionado al tema de trabajo de la tesis, antecedentes, objetivos e hipótesis. En el Capítulo 2 se presentan las características del área de estudio, una reseña histórica de la conformación del Cinturón Hortícola y las formas actuales de manejo. En el Capítulo 3 se describe y analiza la biodiversidad presente en los sistemas hortícolas del área de estudio y la percepción que los agricultores tienen acerca de la vegetación espontánea. En el Capítulo 4 se caracteriza el espectro de fincas del Cinturón Hortícola para establecer si existe un gradiente de intensidad de manejo que va de sistemas convencionales intensivos a orgánicos, con intermedios denominados de bajos insumos. Se presupone que otras variables además del uso o no de químicos definen el manejo hortícola platense. En los siguientes capítulos (Capítulos 5 y 6 ) se analiza la diversidad de espontáneas a diferentes escalas espaciales $(\alpha, \beta, \gamma)$ y se evalúa el efecto del manejo sobre la misma. Para ello, se tienen en cuenta las variables de manejo más relevantes identificadas en el Capítulo 4. Las distintas hipótesis consideradas en las diferentes escalas, indagan sobre la posibilidad de que los cultivos y fincas orgánicas presenten una mayor diversidad, composición florística y otras características respecto

Diversidad Vegetal Espontánea en Agroecosistemas Hortícolas de La Plata y su relación con diferentes estilos de Agricultura: Importancia 
de las convencionales. El Capítulo 5 se orienta a determinar si la diversidad a escala parcela de cultivo $(\alpha)$ disminuye con el aumento de la intensidad de las prácticas de manejo. Se plantea que el uso de herbicidas y la tolerancia del agricultor a las espontáneas podrían ser las variables más relevantes. Este capítulo analiza además la posibilidad de cambios composicionales entre manejos diferentes, esperando que sea más rica la composición florística en los orgánicos. En el Capítulo 6 se evalúa el efecto del manejo sobre la diversidad entre cultivos diferentes $(\beta)$ y en el área total cultivada de las fincas $(\gamma)$. Se busca establecer si la mayor heterogeneidad de hábitat determina una mayor diversidad de especies en estas escalas de análisis, que se traducirían en un mayor número de especies exclusivas, perennes, nativas y dicotiledóneas en los sistemas orgánicos. Asimismo se orienta a determinar el grado de influencia del paisaje sobre la diversidad de espontáneas de las fincas. Se evalúa si un aumento en la heterogeneidad de hábitats del entorno que rodea a las fincas se asocia con una mayor diversidad de espontáneas. En el Capítulo 7 o discusión general, los resultados obtenidos de los cuatro capítulos previos en la zona de estudio, se discuten en relación con la teoría ecológica aplicada a los agroecosistemas y el estado actual de conocimiento en el marco de la agricultura sustentable. Se destacan los aspectos novedosos, propuestas y alternativas para el diseño y manejo de agroecosistemas que garanticen la conservación de la biodiversidad.

\subsection{BIBLIOGRAFÍA}

Albrecht H. 1995. Changes in the arable weed flora of Germany during the last five decades. Simposio 9th EWRS, Budapest 1995. 1: 41-48.

Albrecht H. 2003. Suitability of arable weeds as indicator organisms to evaluate species conservation effects of management in agricultural ecosystems. Agriculture, Ecosystems and Environment 98: 201-211.

Altieri M. 1988. El impacto, usos y rol ecológico de las malezas en los agroecosistemas. En: Altieri MA \& Liebman MZ (Eds.). Weed management in Agroecosystems. Ecological Approaches. CRC Press, Boca Raton, Florida. 1-6 p.

Altieri M. 1997. Ecología y manejo de malezas. En: Altieri MA (Ed.). Agroecología, bases científicas para una agricultura sustentable. CLADES. 14: 391-418.

Altieri MA. 1999. The ecological role of biodiversity in agroecosystems. Agriculture, Ecosystems and Environment 74: 19-31.

Altieri M \& Nicholls Cl. 1994. Biodiversity and pest management in agroecosystems. Haworth Press, New York. 185 p.

Diversidad Vegetal Espontánea en Agroecosistemas Hortícolas de La Plata y su relación con diferentes estilos de Agricultura: Importancia 
Altieri MA \& Nicholls CA. 1999. Biodiversity, Ecosystem Function, and Insect Management in Agricultural Systems. En: Biodiversity in Agroecosystems WW Collins \& CO Qualset (Eds.), CRC Press LLC. 5: 69-84.

Altieri M \& Nicholls Cl. 2000. Agroecología. Teoría y práctica para una agricultura sustentable. Serie Textos Básicos para la Formación Ambiental. PNUMA. Red de Formación Ambiental para América Latina y el Caribe, México. 250 p.

Andersson TN \& Milberg P. 1998. Weed flora and the relative importance of site, crop, crop rotation, and nitrogen. Weed Science 46: 30-38.

Armengot L, Sans FX, Fischer C, Florhe A, José-Maria L, Tscharntke T \& Thiers C. 2012. The $\beta$ diversity of arable weed communities on organic and conventional cereal farms in two contrasting regions. Applied Vegetation Science 15: 571-579.

Baessler C \& Klotz S. 2006. Effects of changes in agricultural land-use on landscape structure and arable weed vegetation over the last 50 years. Agriculture, Ecosystems and Environment 11(1-4) 53-50.

Balezentiene L. 2011. Alpha diversity of differently managed agroecosystems assessed at a habitat scale. Polish Journal of Environment Studies: 1387-1394.

Banks J. 2004. Divided Culture: Integrating and Conservation Biology Agriculture Environmental Science, Interdisciplinary Arts and Sciences 2(10): 537-545. Disponible en: http://digitalcommons.tacoma.uw.edu/ias_pub/3.

Batáry P, Baldi A, Kleijn D \& Tscharntke T. 2011. Landscape-moderated biodiversity effect of agri-enviromental management: a meta-analysis. Proceeding of the Royal Society 278 : 1894-1902.

Bavec M \& Bavec F. 2015. Impact of organic farming and biodiversity. En: Blanco J \& Yueh-Hisin Lo \& Roy S (Eds). Biodiversity and ecosystems. Linking structure and function, Intech. 658 p.

Bengtsson J, Ahnstrom J \& Weibull AC. 2005. The effects of organic agriculture on biodiversity and abundance: a meta-analysis. Journal of Applied Ecology 42: 261269.

Benton TG, Vickery JA \& Wilson JD. 2003. Farmland biodiversity: is habitat heterogeneity the key? Trend in ecology and evolution 18(4): 182-188.

Blake RJ, Woodcock BA, Ramsay AJ, Pilgrim ES, Brown VK, Tallowin JR \& Potts SG 2011. Novel margin management to enhance Auchenorrhyncha biodiversity in intensive grasslands. Agriculture, Ecosystems and Environment 140: 506-513.

Brooks DJ Bater H. Jones \& PA Shah. 1995. Invertebrate and Weed Seed Food-sources for Birds in Organic and Conventional Farming Systems. BTO Informe $N^{0} 154$, BTO, Thetford.

Brussaard L, Caron P, Campbell B, Lipper L, Mainka S, Rabbinge R, Babin D \& Pullman M. 2010. Reconciling biodiversity conservation and food security: scientific challenges for a new agriculture. Current Opinion in Environmental Sustainability 2: 34-42. 
Burel F \& Baudry J. 2002. Ecología del Paisaje. Conceptos, métodos y aplicaciones. Ediciones Mundi prensa, Madrid. 353 p.

CASAFE. 2012. Guía de Productos Fitosanitarios para la Argentina. Cámara de sanidad agropecuaria y fertilizantes de la República Argentina, Buenos Aires.

Chase JM. 2003. Community assembly: when should history matter? Oecologia 136: 489-498.

Clergue B, Amiaud FP, Lasserre-Joulin F \& Plantureux S. 2005. Biodiversity: function and assessment in agricultural areas. A review. Agronomy for Sustainable Development 25: $1-15$.

Collins W \&. Qualset O.1999. Biodiversity in Agroecosystems CRC Press, Boca Raton, Florida. $334 \mathrm{p}$.

Cornell HV \& Lawton JH. 1992. Species interactions, local and regional processes, and limits to the richness of ecological communities: A theoretical perspective. Journal of Animal Ecology 61: 1-12.

de Bello F, Lavorel S, Díaz S, Harrington R, Cornelissen J, Bardgett R, Berg M, Cipriotti P, Feld C, Hering D, Martins da Silva P, Potts S, Sandin L, Sousa J, Storkey J, Wardle D \& Harrison P. 2010. Towards an assessment of multiple ecosystem processes and services via functional traits. Biodiversity \& Conservation 19: 2873-2893.

de la Fuente EB \& Suarez SA. 2008. Problemas ambientales asociados a la actividad humana: la agricultura. Ecología Austral. 18: 239-252.

de la Fuente EB, Suarez SA, Ghersa CM \& León RJC. 1999. Soybean weed comunities: relationships whit cultural history and crop yield. Agronomy Journal 91: 234-241.

Diamond JM. 1975. Assembly of species communities. Ecology and evolution of communities. En: Cody ML \& Diamond JM (Eds.). Harvard University Press, Cambridge, USA. 342-444 p.

di Castri F \& Younes T. 1996. Biodiversity, science and development: towards a new partnership. Wallingford, CAB International. $646 \mathrm{p}$.

Edesi L, Jarvan M, Adamson A, Lauringson E \& Kuht J. 2012. Weed species diversity and community composition in conventional and organic farming: a five-year experiment. Zemdirbyste 99: 339-346. Disponible en: http://www.vkm.no/dav/dda3cb51f7.pdf

Evans LT. 1993. Crop Evolution, Adaptation and Yield Evolution. Cambridge University Press, Cambridge. 500 p.

FAO. 2013. Stadistical yearbook 2013. World Food and Agriculture, FAO. Roma. 289 pp. Disponible en http://www.fao.org/docrep/018/i3107e/i3107e00.htm. Último acceso: diciembre de 2013.

FAO. 2015. Construyendo una visión común para la agricultura y alimentación sostenibles. Informe de la Organización de las Naciones Unidas para la Alimentación y la Agricultura. $5 p$.

Diversidad Vegetal Espontánea en Agroecosistemas Hortícolas de La Plata y su relación con diferentes estilos de Agricultura: Importancia 
Fernández, V \& Marasas M. 2015. Análisis comparativo de la riqueza de especies y familias botánicas en sistemas de producción hortícola familiar del Cordón Hortícola de La Plata (CHLP), Provincia de Buenos Aires, Argentina. Su importancia para la transición agroecológica. Revista de la Facultad de Agronomía 114(1): 15-29.

Fischer J, Abson DJ, Butsic V, Chappell MJ, Ekroos J, Hanspach, J \& Wehrden H. 2014. Land sparing versus land sharing: moving forward. Conservation Letters 7(3): 149157.

Foley JA, DeFries R, Asner GP, Barford C, Bonan G, Carpenter R, Chapin FS, Coe MT, Daily GC, Gibbs HK, Helkowski JH, Holloway T, Howard EA, Kucharik CJ, Monfreda C, Patz JA, Prentice IC, Ramankutty N \& Zinder PK. 2005. Global Consequences of Land Use. Science 309: 570-574.

Forman RTT. 1995. Land Mosaics. The Ecology of Landscapes and Regions. Cambridge University Press, Cambridge, UK. 632 p.

Froud-Willams RJ. 1987. Changes in weed flora with different tillage and agronomic management systems. En: Altieri M \& Liebman M (Eds.) Weed management in agro ecosystems: ecological approaches. CRC Press, Inc, Florida. 213-236 p.

Gaba S, Reboud X \& Fried G. 2016. Agroecology and conservation of weed diversity in agricultural lands. Botany Letters 163(4): 351-354.

Gabriel D, Sait SM, Hodgson JA, Schmutz U, Kunin WE \& Benton TG. 2010. Scale matters: the impact of organic farming on biodiversity at different spatial scales. Ecology Letters 13(7): 858-869.

Gabriel D, Sait SM, Kunin WE \& Benton TG. 2013. Food production vs. biodiversity: Comparing organic and conventional agriculture. Journal of Applied Ecology 50: 355364.

Garbach K, Milder JC, Montenegro M, Karp DS \& DeClerck FAJ. 2014. Biodiversity and Ecosystem Services in Agroecosystems. Encyclopedia of Agriculture and Food Systems 2: 21-40.

Gardner SM \& Brown RW. 1998. Review of the Comparative Effects of Organic Farming on Biodiversity. MAFF, London.

Ghersa CM \& León RJC. 1999. Successional changes in agroecosystems of the Rolling Pampa. En: Walker LR (Ed.) Ecosystems of the World 16: Ecosystems of disturbed ground. Elsevier 20: 487-502.

Ghersa CM \& Martinez-Ghersa MA. 2000. Ecological correlates of weed seed size and persistence in the soil under different tilling systems: implications for weed management. Field Crops Research 67: 141-148.

Gliessman S. 2001. Agroecología. Processos ecológicos em agricultura sustentável. Segunda Edición. Editora da Universidade Río Grande do Sul, Brasil. 652 pp.

González AD, Janke R \& Rapoport E. 2003. Valor nutricional de las malezas comestibles. Ciencia Hoy 13(76): 40-47.

Grime JP. 1979. Plant strategies and vegetation process. Chichester, New York, John Wiley. 222 p. 
Halffer G, Morello J, Matteucci DS \& Solbrig OT. 1999. La biodiversidad y el uso de la tierra. En: Mateucci SD, Solbrig OT, Morello J, Halffter G (Eds.) Biodiversidad y uso de la tierra. Conceptos y ejemplos de Latinoamérica. Eudeba, Universidad de Buenos Aires.17-27 pp.

Harlan JR 1976. Las plantas y los animales que alimentan al hombre. En: Investigación y Ciencia, edición en español de Scientific American n²: 65-74p.

Harlan JR. 1992. Crops and Man (2da. Ed.). American Society of Agronomy and Crop Science Society of America, Madison, Wisconsin, USA. 284 p.

Henckel L, Borger L, Meiss, Gaba S \& Bretagnolle V. 2015. Organic field sustain weed metacommunity in farmland landscapes. Proceeding of the Royal Society B: 282: 20150002.

Hole DG, Perkins AJ, Wilson JD, Alexander IH, Grice PV \& Evans AD. 2005. Does organic farming benefit biodiversity? Biological Conservation 122: 113-130.

Huston MA. 1994. Biological Diversity: The Coexistence of Species on Changing Landscape. Cambridge University Press, Cambridge. 681 p.

Hutchison GE. 1953. The concept of pattern in ecology. Proceedings of the Academy the Natural Science 105:1-12.

Hyvönen T \& Salonen J. 2002. Weed species diversity and community composition in cropping practices at two intensity levels - a six-year experiment. Plant Ecology 159: 73-81.

Hyvönen T, Ketoja E, Salonen J, Jalli H \& Tiainen J. 2003. Weed species diversity and community composition in organic and conventional cropping of spring cereals. Agriculture, Ecosystems and Environment 97: 131-149.

IFOAM. 2007. Normas de IFOAM para la produccción y el procesamiento orgánicos. Disponible en https://www.ifoam.bio/en/our-library.

Jackson LE, Pascual U \& Hodgkin T. 2007. Utilizing and conserving agrobiodiversity in agricultural landscapes. Agriculture, Ecosystems and Environment 121: 196-210.

Jarvis DI, Padoch C \& Cooper HD. 2011. Manejo de la biodiversidad en los Ecosistemas Agrícolas. Biodiversity Internacional, Roma. 503 p.

Kleijn D, Kohler F, Báldi A, Batáry P, Concepción ED, Clough Y, Díaz M, Gabriel D, Holzchuh A, Knop E, Kovács A, Marshall EJP, Tscharnke T \& Verhults J. 2009. On the relationship between farmland biodiversity and land-use intensity in Europe. Proceedings of the Royal Society B: 276: 903-909.

Koleff P \& Gaston KJ. 2002. The relationships between local and regional species richness and spatial turnover. Global Ecology and Biogeography 11: 363-375.

Krauss J, Steffan-Dewenter I \& Tscharntke T. 2003. How does landscape context contribute to effects of habitat fragmentation on diversity and population density of butterflies? Journal of Biogeography 30: 889-900.

Krebs JR, Wilson JD, Bradbury RB \& Siriwardena GM. 1999. The second silent spring? Nature 400: 611-612.

Diversidad Vegetal Espontánea en Agroecosistemas Hortícolas de La Plata y su relación con diferentes estilos de Agricultura: Importancia 
Lampkin N. 2001. Certified and policy supported organic and in conversion land area in Europe 1985-2001. Disponible en: http://www.organic.aber.ac.uk/stats.html. Ultimo acceso: diciembre de 2006.

MacArthur RH. 1965. Patterns of species diversity. Biological Review 40: 510-533.

Magurran AE. 1988. Ecological Diversity and Its Measurement. Pinceton University Press, New Jersey. $179 \mathrm{p}$.

Marasas M, Fernandez V, Baloriani G, Cap G, Larrosa C \& Rouaux J. 2011. Estudio de la Agrobiodiversidad en Sistemas de Producción Hortícola Familiar. Buenos Aires. Argentina. Cuadernos de Agroecologia 6(2): 1-4.

Marshall EJP, Brown VK, Boatman ND, Lutman PJW \& Squire GR. 2002. The impact of herbicides on weed abundance and biodiversity. DEFRA, Informe PN0940.

Marshall EJP, Brown VK, Boatman ND \& Lutman PJW. 2003. The role of weeds in supporting biological diversity within crop fields. Weed Research 43: 77-89.

Martinez-Ghersa MA, Ghersa CM \& Satorre EH. 2000. Coevolution of agricultural systems and their weed companions: implications for research. Field Crop Research 67: 181-190.

Marzocca A. 1976. Manual de malezas, 4ta edición actualizada por OJ Mársico y O. Hemisferio Sur SA, Buenos Aires. 564 p.

Matson PA, Parton WJ, Power AG \& Swif MJ. 1997. Agricultural intensification and ecosystem properties. Science 277: 504-509.

McLaughlin A \& Mineau P. 1995. The impact of agricultural practices on biodiversity. Agriculture, Ecosystems \& Environment 55: 201-212.

Moreby SJ, Aebischer NJ, Southway SE \& Sotherton NW. 1994. A comparison of the flora and the arthropod fauna of organically and conventionally grown winter wheat in southern England. Annals Applied Biology 125: 13-27.

Noss RF. 1990. Indicators for Monitoring Biodiversity: A Hierarchical Approach. Conservation Biology 4: 355-364.

Paleologos MF, CC Flores, SJ Sarandón, SA Stupino \& MM Bonicatto. 2008. Abundancia y diversidad de la entomofauna asociada a ambientes seminaturales en fincas horticolas de La Plata, Buenos Aires, Argentina. Revista Brasileira de Agroecología 3(1): 28-40.

Palmer MW \& Maurer T. 1997. Does diversity beget diversity?. A case study of crops and weed: Journal of Vegetation Science 8: 235-240.

Pereyra, LC \& Moreno CE. 2013. Divide y vencerás: revisión de métodos para la partición de la diversidad regional de especies en sus componentes alfa y beta Revista Chilena de Historia Natural 86: 231-239.

Petit S, Alignier A, Colbach N, Joannon A, Coeur D \& Thenail C. 2013. Weed dispersal by farming at various spatial scales. A review. Agronomy for Sustainable Development 33(1): 205-217.

Diversidad Vegetal Espontánea en Agroecosistemas Hortícolas de La Plata y su relación con diferentes estilos de Agricultura: Importancia 
Petit S, Gaba S, Grison AN, Meiss H, Simmoneau B, Jolain N \& Bretagnolle V. 2016. Landscape scale management affects weed richness but now weed abundance in whinter wheat fields. Agriculture, Ecosystems, and Environment 223: 41-47.

Pimentel D, Stachow U, Takacs DA, Brubaker HW, Dumas AR, Meaney JJ, O'Neil JAS, Onsi DE \& Corzilius DB. 1992. Conserving biological diversity in agricultural/forestry systems. BioScience 42: 354-362.

Pochettino L. 2005. Congreso Argentino de Inmigración. Verduras en Europa, yuyos en América. Prácticas y conocimientos sobre malezas comestibles. Actas Congreso Argentino de inmigración, IV Congreso de Historia de los pueblos de la provincia de Santa Fe. 24p.

Poggio S. 2005. Structure of weed communities occurring in monoculture and intercropping of field pea and barley. Agriculture, Ecosystems and Environment 19:4858.

Poggio S. 2007. Relaciones entre la diversidad de especies vegetales y la heterogenidad espacial del paisaje agrícola pampeano. Tesis de Doctorado en Ciencias Agropecuarias. Facultad de Agronomía, Universidad de Buenos Aires, Argentina.125 p.

Poggio S. 2012. Cambios florísticos en comunidades de malezas: un marco conceptual basado en reglas de ensamblaje. Ecología Austral 22: 150- 158.

Poggio SL, Chaneton EJ \& Ghersa CM. 2013. The arable plant diversity of intensively managed farmland: Effects of field position and crop type at local and landscape scales. Agriculture, Ecosystems \& Environment 166: 55-64.

Puricelli E, Faccini D, Nisenshon L \& Tuesca D. 2012. Weed cover, frequency and diversity in field plots and edges in the soybean central region of Argentina. Agricultural Science 3(5): 631-639.

Pysek P \& Leps J. 1991. Response of a weed community to nitrogen fertilization: a multivariate analysis. Journal of Vegetation Science 2: 237-244.

Radosevich SR, Holt J \& Ghersa CM. 2007. Ecology of weeds and invasive plants. Relationship to agriculture and natural resources management. 3ra edition. John Wiley \& Sons: Nueva York. 454 p.

Rahmamn G. 2011. Biodiversity and Organic farming: What do we Know?. Agriculture and Forestry Research 61(3): 189-208.

Rapoport EH, Marzocca A \& Drausal BS. 2009. Malezas comestibles del Cono Sur y otras partes del planeta. Ediciones Instituto Nacional de Tecnología Agropecuaria, Bariloche, Argentina. 216 p.

Ricklefs RE \& Schluter. 1993. Species diversity in ecological communities. University of Chicago Press: Chicago. 575 p.

Rodríguez P \& Vázquez-Domínguez E. 2003. Escalas y diversidad de especies. En: Morrone JJ \& Llorente J (Eds.). Una perspectiva latinoamericana de la biogeografía. Facultad de Ciencias, UNAM, México. 109-114.

Diversidad Vegetal Espontánea en Agroecosistemas Hortícolas de La Plata y su relación con diferentes estilos de Agricultura: Importancia 
Rodríguez Lagreca J. 2006. Las malezas y el agroecosistema. Unidad de Malezas, Departamento de Protección Vegetal, Facultad de Agronomía, Universidad de la República Oriental del Uruguay. 30 p.

Roschewitz I, Gabriel D, Tscharntke T \& Thies C. 2005. The effects of landscape complexity on arable weed arable diversity in organic and conventional farming. Journal of Applied Ecology 42(5): 873-882.

Rosenzweig ML. 1995. Species diversity in space and time. Cambridge: Cambridge University Press. 436 p.

Rydberg N \& Milberg P. 2000. A survey of weeds in organic farming in Sweden. Biological Agriculture \& Horticulture 18: 175-185.

Sarandón SJ. 2002. El agroecosistema: un sistema natural modificado. Similitudes y diferencias entre ecosistemas naturales y agroecosistemas. En: Sarandón SJ (Ed.). Agroecología. El camino hacia una agricultura sustentable. Ediciones Científicas Americanas, La Plata. 6: 119-134.

Sarandón SJ, Zuluaga MS, Cieza R, Gómez C, Janjetic L \& Negrete E. 2006. Evaluación de la sustentabilidad de sistemas agrícolas de fincas en Misiones, Argentina, mediante el uso de indicadores. Revista Agroecología 1: 19-28.

Sarandón SJ. 2009. Biodiversidad, agrobiodiversidad y agricultura sustentable: Análisis del Convenio sobre Diversidad Biológica. En: Tomas Leon Siccard, Miguel A. Altieri (Eds.). Vertientes del pensamiento agroecológico: fundamentos y aplicaciones., IDEAS 21, Sociedad Científica Latinoamericana de Agroecología (SOCLA), Universidad Nacional de Colombia, Opciones Graficas Editores, Bogotá, Colombia, 4: 105-130.

Sarr DA, Hibbs DE \& Huston MA. 2005. A Hierarchical Perspective of Plant Diversity. The Quarterly Review of Biology 80(2): 187-212.

SCDB. 2008. Secretaría del Convenio sobre la Diversidad Biológica. La biodiversidad y la agricultura. Salvaguardando la biodiversidad y asegurando alimentación para el mundo, Montreal. 56 pp. Disponible en: https://www.cbd.int/doc/bioday/2008/ibd2008-booklet-es.pdf.

Schmitzberger I, Wrbka T, Steurer B, Aschenbrenner, G, Peterseil J \& Zechmeister H. 2005. How farming styles influence biodiversity maintenance in Austrian agricultural landscapes. Agriculture, Ecosystems and Environment 108: 274-290.

Shepherd M, Pearce B, Cormack B, Phillipps L, Cuttle S, Bhogal A, Costigan P \& Unwin R. 2003. An assessment of the environmental impacts of organic farming. Una revisión para Defra-funded project OF0405. ADAS Consultora Ltd. Mansfield. 80 p. Disponible en: http://orgprints.org/6784/2/OF0405_909_TRP.pdf.

Shmida A \& Wilson MV. 1985. Biological determinants of species diversity. Journal of Biogeography 12: 1-20.

Solbrig O. 1999. Observaciones sobre biodiversidad y desarrollo agrícola. En: Mateucci SD, Solbrig OT, Morello J \& Halffter G (Eds.) Biodiversidad y uso de la tierra. Conceptos y ejemplos de Latinoamérica, Eudeba, Buenos Aires. 2: 29- 40.

Diversidad Vegetal Espontánea en Agroecosistemas Hortícolas de La Plata y su relación con diferentes estilos de Agricultura: Importancia para la sustentabilidad 
Stupino SA, AC Ferreira, JL Frangi y SJ Sarandón. 2007. Agrobiodiversidad vegetal en sistemas hortícolas orgánicos y convencionales (La Plata, Buenos Aires, Argentina). Revista Brasileira de Agroecología, Asociación Brasilera de Agroecología, Con referato. ISSN 1980-9735. Edición especial: Resúmenes del II Congreso Brasileiro de Agroecología, 22 al 25 de Noviembre de 2004. Vol 2(1): 339-342.

Stupino SA, Frangi JL, Sarandón SJ, Arturi MF \& Ferreira AC. 2008. Plant diversity in two farms under organic and conventional management in La Plata, Argentina. A case study. Revista Brasilera de Agroecología, Asociación Brasilera de Agroecología 3(3): 24-35.

Stupino SA, lermanó MJ, Gargoloff NA \& Bonicatto MM. 2014. La biodiversidad en los agroecosistemas. En: Sarandón J \& Flores C (Eds.). Agroecología: Bases teóricas para el diseño y manejo de agroecosistemas sustentables. Colección de libros de cátedra, Secretaria de Asuntos Académicos-Edulp, UNLP.

Suárez SA, de la Fuente EB, Ghersa CM \& RJC. León. 2001. Weed community as an indicator of summer crop yield and site quality. Agronomy Journal 93: 524-530.

Sutcliffe OL \& Kay QON. 2000. Changes in the arable flora of central southern England since the 1960s. Biological Conservation 93:1-8.

Swift MJ \& Anderson JM.1994. Biodiversity and ecosystem function in agricultural systems. En: Schulze ED \& Mooney HA (Eds.). Biodiversity and Ecosystem Function. SpringerVerlag, Berlin. 15-42 pp.

Swift MJ, MN Izac \& van Noordwijk M. 2004. Biodiversity and ecosystem services in agricultural landscapes—are we asking the right questions?. Agriculture, Ecosystems and Environment 104: 113-134.

Tang L, Wan K, Chuanpeng C \& Chen F. 2013. Effect of fertilization patterns on the assemblage of weed communities in an upland winter wheat field. Journal of Plant Ecology 7(1): 39-50.

Terradas J. 2001. De la ecofisiología de las plantas a la dinámica de comunidades y paisajes. Omega, Barcelona. 760 p.

Tilman D.1999. Global environmental impacts of agricultural expansion: The need for sustainable and efficient practices. Proceedings of the National Academy of Sciences of the United States of America 96:5995-6000.

Tscharntke T, Kleijn AM, Kruess A, Steffan- Dewenter I \& Thies C. 2005. Landscape perspectives on agricultural intensification and biodiversity-ecosystem service management. Ecology Letters 8: 857-874.

Tscharntke T, Clough Y, Wanger T, Jackson L, Motzke I, Perfecto I, Vandermeer J \& Whitbread A. 2012. Global food security, biodiversity conservation and the future of agricultural intensification. Biological Conservation 151(1): 53-59.

Tuck SL, Winqvist C, Mota F, Ahnström J, Turnbull LA \& Bengtsson J. 2014. Land-use intensity and the effects of organic farming on biodiversity: a hierarchical metaanalysis. Journal of Applied Ecology 51: 746-755. 
Tuomisto H. 2010. A diversity of beta diversities: straightening up a concept gone awry. Part 1. Defining beta diversity as a function of alpha and gamma diversity. Ecography 33: 2-22.

UNEP. 1997. The Biodiversity Agenda. Decisions from the third Meeting of the Conference of the Parties to the Convention on Biological Diversity. 2da. ed., Buenos Aires, Argentina, Noviembre de 1996. 116 p.

UNEP. 2000. The Biodiversity Agenda. Decisiones adoptadas por la conferencia de las partes en el convenio sobre la diversidad biológica en su quinta reunión. Apéndice Nairobi, 15-26 de Mayo de 2000.

Unwin R, Bell B, Shepherd M, Webb J, Keatinge R \& Bailey S. 1995. The Effect of Organic Farming Systems on Aspects of the Environment - A review prepared for Agricultural Resources Policy Division of the Ministry of Agriculture, Fisheries and Food, MAFF, London.114 p.

Vandermeer J \& Perfecto I. 1995. Breakfast of biodiversity: the truth about rainforest destruction. Food First Books, Oakland. 185 p.

Vandermeer J, van Noordwijk M, Anderson J, Ong C \& Perfecto I. 1998. Global change and multi-species agroecosystems: concepts and issues. Agriculture, Ecosystems \& Environment 67(1): 1-22.

van Elsen T. 2000. Species diversity as a task for organic agriculture in Europe. Agriculture, Ecosystems and Environment 77: 101-109.

van der Ploeg JD. 1993. Rural sociology and the new agrarian question: A perspective from The Netherlands. Sociologia Ruralis 33(2): 240-260.

Weibull AC \& Ostman O. 2003. Species composition in agroecosystems: the effect of landscape habitat Basic and Applied Ecology 4: 349-361.

Weibull AC, Ostman O \& Granqvist A. 2003. Species richness in agroecosystems: the effect of landscape, habitat and farm management. Biodiversity and Conservation 12: 1335-1355.

Wilson PJ. 1993. Conserving Britain's cornfield flowers. En: Proceedings 1993 Brighton Crop Protection Conference-weed, Brighton, UK.22-25 de noviembre de 1993, 411$416 \mathrm{p}$.

Winqvist C, Ahnström J, Bengtsson J. 2012. Effects of organic farming on biodiversity and ecosystems services: taking landscape complexity into account. Annals of the New York Academy of Sciences 1249: 191-203.

Whittaker RH. 1960. Vegetation of the Siskiyou Mountains. Oregon and California. Ecological Monographs 30: 279-338.

Whittaker RH. 1977. Evolution of species diversity in land communities. Evolutionary Biology 10: 1-67.

Whittaker RJ, Willis KJ \& Field R. 2001. Scale and species richness: Towards a general, hierarchical theory of species diversity. Journal of Biogeography 28: 453-470. 
Woodcock BA \& Pywell RF. 2010. Effects of vegetation structure and floristic diversity on detritivore, herbivore and predatory invertebrates within calcareous grasslands. Biodiversity \& Conservation 19: 81-95. 


\section{CAPÍTULO 2}

\section{EL CINTURÓN HORTÍCOLA Y SU HISTORIA DE USO AGRÍCOLA}

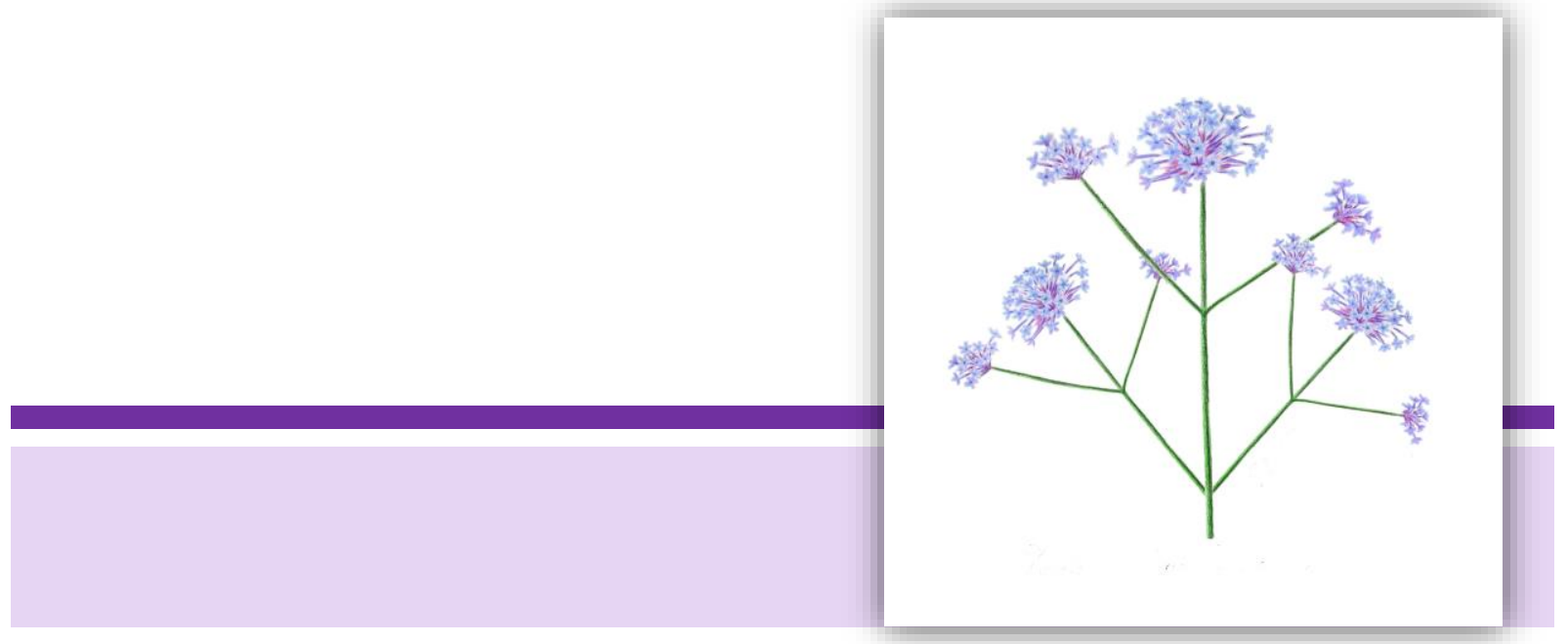




\section{CAPÍTULO 2}

Con los pies en la tierra y la mirada en el cielo. Juan B. Terán (1880-1938). Abogado e historiador

\section{EL CINTURÓN HORTÍCOLA Y SU HISTORIA DE USO AGRÍCOLA}

\subsection{Características de la ciudad de La Plata}

El partido de La Plata se ubica en el noreste del territorio bonaerense y limita al este con Berisso y Ensenada, al sudeste con Magdalena, al sur con Brandsen, al sudoeste con San Vicente y al norte con Berazategui y Florencio Varela (Figura 2.1).

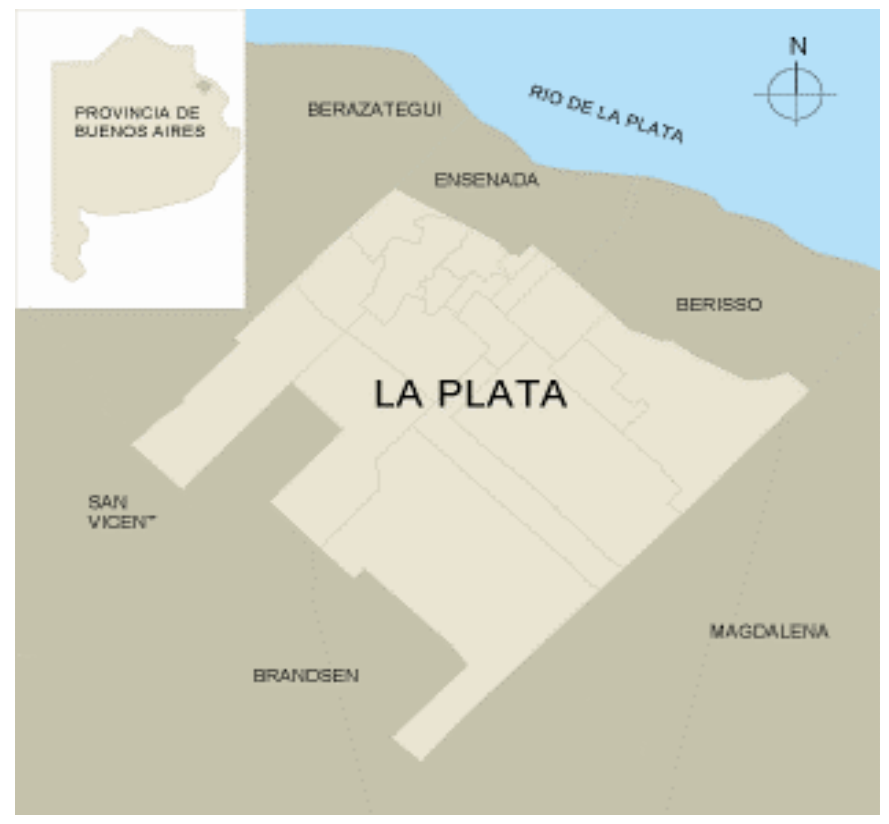

Figura 2.1. Ubicación geográfica del partido de La Plata y localidades vecinas. Fuente: www.estadistica.laplata.gov.ar.

Diversidad Vegetal Espontánea en Agroecosistemas Hortícolas de La Plata y su relación con diferentes estilos de Agricultura: Importancia 
Ocupa una superficie de 194.000 ha, sobre un relieve de llanura con ondulaciones leves. Los suelos son típicamente Argiudoles, caracterizados por un horizonte subsuperficial de acumulación de arcillas, y aptos para las actividades agrícolas. En esta zona la temperatura anual media es de $16.2^{\circ} \mathrm{C}$, el promedio de lluvias de $1096 \mathrm{~mm}$. Por su cercanía al río de la Plata la humedad tiende a ser abundante, siendo la media anual de 77,6 \% (Hurtado et al., 2006).

\subsection{Conformación del cinturón verde y el origen de la horticultura}

La horticultura es una de las actividades agrícolas más importante de la provincia de Buenos Aires y está especialmente desarrollada en el área periurbana del municipio de La Plata ( $34^{\circ} 8 \mathrm{~S} 57^{\circ} 54 \mathrm{~W}$ ). Los suelos donde se desarrolla la actividad son Argiudoles verticos y Hapludertes típicos, caracterizados por una alta fertilidad química, alto nivel de materia orgánica y su pH es ligeramente ácido (pH 5,5) (Hurtado et al., 2006; Cuellas, 2017). Los suelos son aptos para la agricultura, aunque condicionados por la permeabilidad moderada a baja y la elevada plasticidad, debido a que poseen un elevado contenido de arcillas expansibles (entre $32-40 \%$ en superficie) que se incrementan en profundidad (Hurtado et al., 2006). Los sedimentos son de origen continental compuestos de material loéssico eólico y fluvial.

Las producciones hortícolas difieren del resto de las producciones pampeanas en que son intensivas en el uso de los factores de la producción (tierra, trabajo y capital), se desarrollan en pequeñas áreas, son más diversificadas y se localizan generalmente en un ámbito periurbano (Archenti et al., 1993; Barsky, 2005). El periurbano es un territorio de "borde" que se desarrolla en el contorno de las ciudades y está en permanente transformación (Barsky, 2005). Forma parte de un paisaje muy heterogéneo de uso productivo, recreativo, residencial y de servicios. Dentro de esta área puede distinguirse el denominado "cinturón verde" que representa al espacio periurbano conformado por una trama de quintas o huertas familiares y otras de características más empresariales que rodean a las grandes ciudades y cuya producción se destina especialmente a verduras de hoja y hortalizas de estación (Benencia et al., 1992; Benencia, 1994; Barsky, 2005; Ringuelet, 2009). Por lo tanto, la agricultura periurbana constituye aquella agricultura intensiva y diversificada que se efectúa alrededor de las ciudades (Barsky, 2014).

Diversidad Vegetal Espontánea en Agroecosistemas Hortícolas de La Plata y su relación con diferentes estilos de Agricultura: Importancia 
Desde la creación de la ciudad de La Plata, a fines del siglo XIX, la región creció rápidamente a partir de actividades administrativas y luego, ya en el siglo $X X$ con el desarrollo de la industria frigorífica y la instalación de petroquímicas. Así se fue creando el "Gran La Plata". Con la ampliación de la ciudad se desarrolló un entorno agrícola ganadero (Ringuelet, 2009). Las pequeñas quintas alrededor de las ciudades fueron la primera manifestación de la horticultura en la Argentina durante los inicios del siglo XIX (Barsky, 2005; Garcia 2010). Este tipo de producción se localizaba en un grupo desarticulado de zonas cercanas al centro urbano, constituyendo islas que luego se multiplicaron hasta constituir el "cinturón verde" (Barsky, 2005; Ringuelet, 2009). La particularidad de la historia de la actividad hortícola reside en la presencia del inmigrante como sujeto social preponderante (Garcia, 2012). Desde el siglo pasado hasta la segunda posguerra se asentaron a través de corrientes migratorias agricultores que aportaron una buena cantidad de los cultivos que se consumían en la región. Los primeros en ocupar los bordes de los espacios urbanizados fueron los Italianos, españoles y en menor medida portugueses (Benencia et al., 2009b; Le Gall \& Garcia, 2010). Posteriormente se dan inmigraciones desde el interior del país y desde de Bolivia (Garat et al., 2009). La producción hortícola se realizaba a campo, con una tecnología muy rudimentaria y en unas 5 hectáreas de superficie (Le Gall \& Garcia, 2010). Los agricultores eran familiares, la mayoría eran propietarios de la tierra, con baja inversión en capital, sin ninguna mejora técnica y alta incidencia del empleo de mano de obra familiar (Benencia, 1994). La tecnología dominante era el arado "mancera" tirado a caballo, deshierbe manual, abonos orgánicos, el riego gravitacional, y los cuidados fitosanitarios por medio de preparados naturales. La producción se basaba en una alta diversidad de cultivos (Garat et al., 2009) que se comercializaban en los mercados mayoristas a través de consignatarios. Hacia la década del 70', comienza a gestarse un periodo de "modernización" con la introducción de maquinaria (1965), el uso de agroquímicos y la introducción de semillas hibridas que posteriormente se integran a un paquete tecnológico hacia los 80' (Simonatto 2000; Lemmi, 2011). La capitalización de un segmento de los agricultor hace que surja un tipo de agricultor y de quinta de mayores dimensiones (20 y 30ha). Hacia la década de los 90', surge una de las transformaciones tecnológicas más importantes que estructura la organización social y productiva de las quintas: el cultivo bajo cubierta (invernáculo) (Benencia et al., 1992; Cieza, 2004; Benencia et al., 2009b; Le Gall \& Garcia, 2010). Dicha tecnología se caracteriza por estructuras de madera recubiertas con polietileno que buscan controlar el ambiente,

Diversidad Vegetal Espontánea en Agroecosistemas Hortícolas de La Plata y su relación con diferentes estilos de Agricultura: Importancia 
permitiendo entonces, una mayor productividad, emplear cultivos fuera de estación, y una diferenciación en la calidad y precio. Esta forma de producir requiere la adopción de tecnologías complementarias al invernáculo como, el uso de semillas mejoradas, riego por goteo y fertirrigación, bromuro de metilo (gas de acción plaguicida) (Benencia et al., 2009b) (Figura 2.2). Esto se complementa con una reducción paulatina de las variedades locales o reemplazo por otras más rentables o con mayores rendimientos (Garat et al., 2009). Como resultado de los cambios de las últimas décadas, el Cinturón Hortícola presenta actualmente una gran heterogeneidad en las formas y lógica de producción, tipos de agricultores, grado de capitalización y canales de comercialización (Hang, 2010; Cieza, 2015).
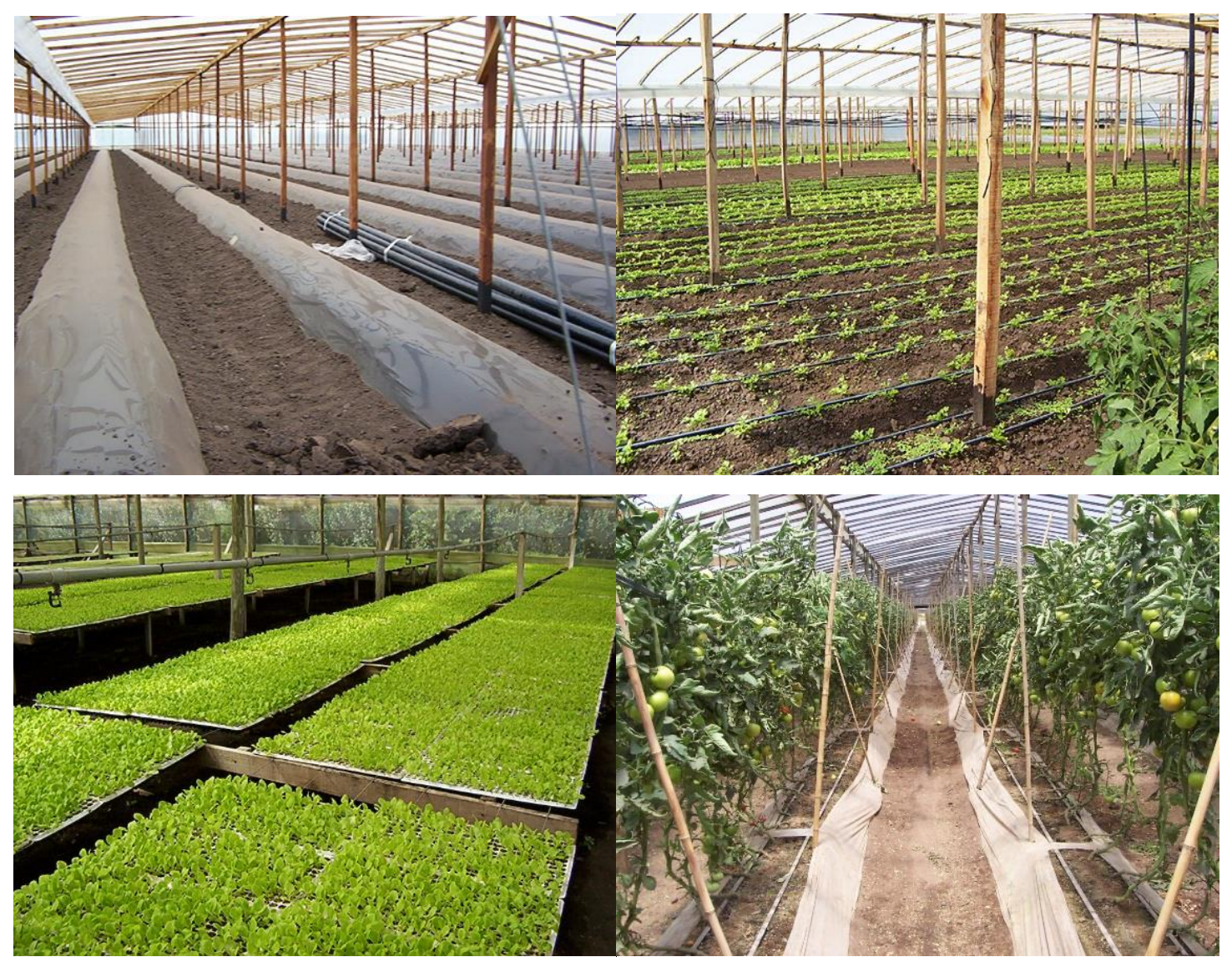

Figura 2.2. Tecnologías complementarias al invernáculo: mangueras para riego por goteo y fertirriego, plantineras con variedades mejoradas y nylon para la aplicación de bromuro de metilo. Fuente: Fotografías de la autora. 


\subsection{Características de la región y las formas de manejo}

El cinturón verde es una franja de territorio de 5 a $12 \mathrm{Km}$ de ancho y 36.580 ha de extensión que representa el $40 \%$ del territorio municipal, un tercio del cual es de carácter productivo, con actividades hortícola y florícola (Bozzano, 2002) (Figura 2.3). Constituye la región más importante del Cinturón Verde Bonaerense, área que rodea a la ciudad de Buenos Aires y alrededores y de la Provincia de Buenos Aires (García, 2012). Según datos obtenidos a partir del Censo Horti-Florícola de Buenos Aires 2005, se concentran en La Plata el $49,2 \%$ de las explotaciones del Cinturón Verde y el $25 \%$ de la Provincia. En esta región la producción se caracteriza básicamente por el cultivo intensivo de productos para el consumo en fresco de la población urbana (Benencia, 1994; Benencia \& Souza Casadhino, 2009a), generando una producción anual de 75.000 Toneladas (Garat et al., 2009).

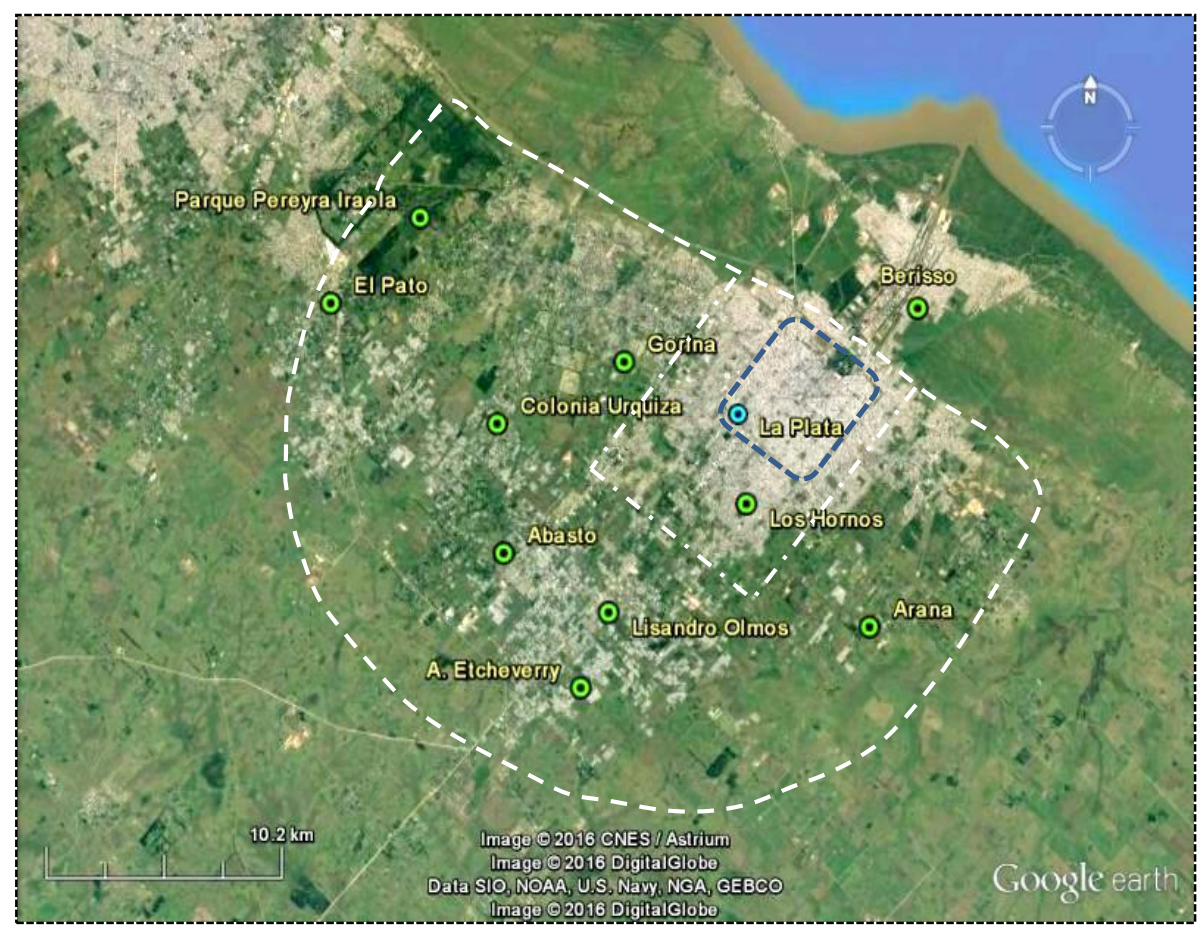

Figura 2.3. Cinturón verde de la ciudad de la Plata. Representa el área del periurbano delimitada por las líneas punteadas blancas. Los círculos indican localidades de referencia donde se localizan las áreas de muestreo (con excepción de Berisso). Fuente: Elaborado por la autora a partir de imagen de Google Earth (2016).

Diversidad Vegetal Espontánea en Agroecosistemas Hortícolas de La Plata y su relación con diferentes estilos de Agricultura: Importancia 
El cinturón cuenta con 738 explotaciones en una superficie un total de 2608 ha, (lo que representa el $41 \%$ de la superficie hortícola del Cinturón Verde Bonaerense, de las cuales 1843 ha se realizan a campo (al aire libre) y 765 ha en invernáculo (bajo cubierta) (CHFBA, 2005; Le Gall \& Garcia, 2010). Las fincas poseen dimensiones pequeñas y medianas (7 ha en promedio) y el manejo se realiza a campo, en invernáculo (bajo cubierta), o mixto (Benencia, 1994). La superficie de los cultivos varían de un surco a 1 ha. En promedio, el invernáculo representa más de una hectárea por explotación (CHFBA, 2005).

Los cultivos bajo cubierta que presentan la mayor superficie cultivada de La Plata son, en orden de importancia: lechuga, tomate, espinaca, pimiento, apio, acelga. Considerando la superficie total (invernáculo más campo) los principales cultivos son lechuga, tomate, acelga, espinaca, choclo y pimiento (CHFBA, 2005) (Figura 2.4 y 2.5).
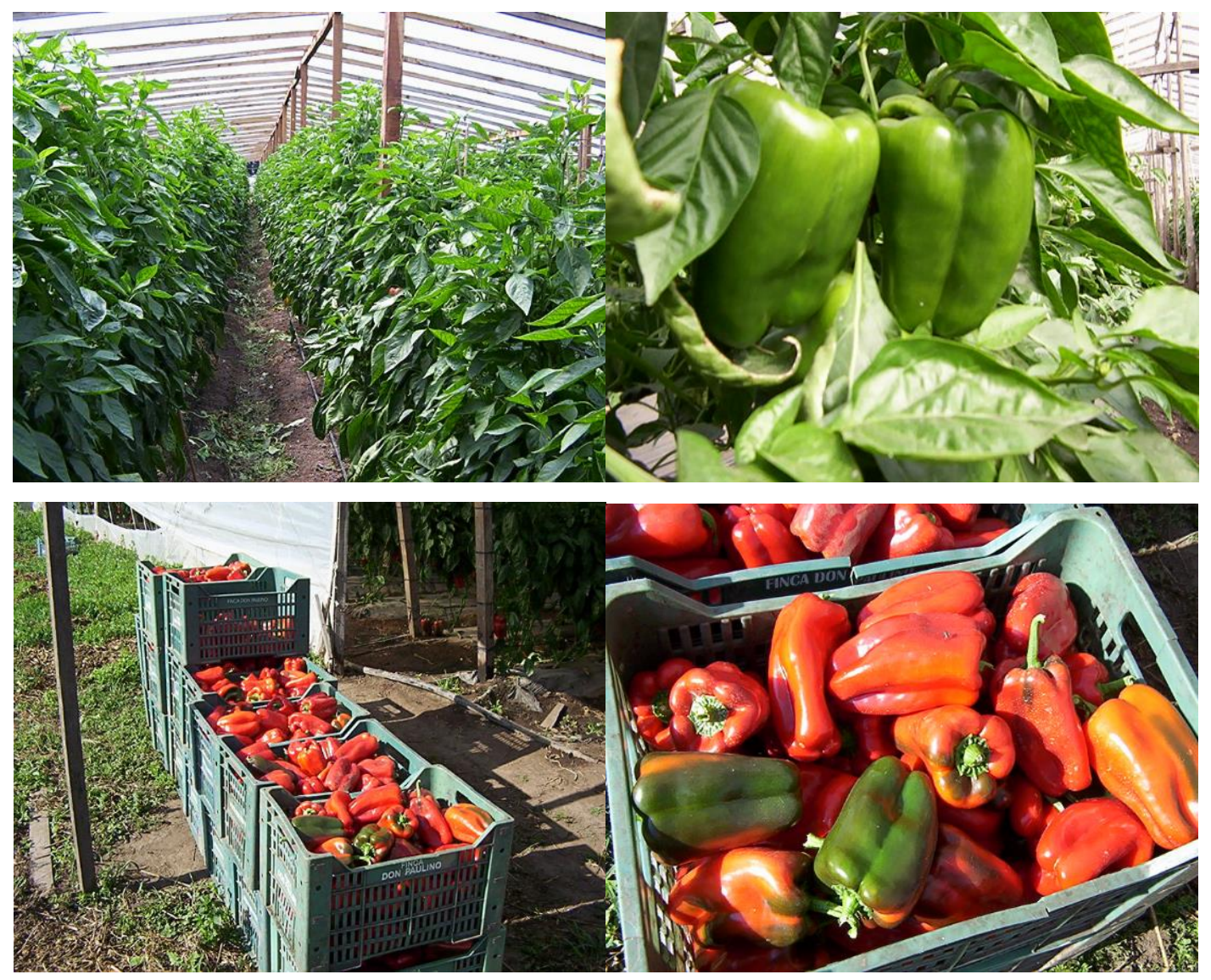

Figura 2.4. Producción del pimiento en invernáculo. 


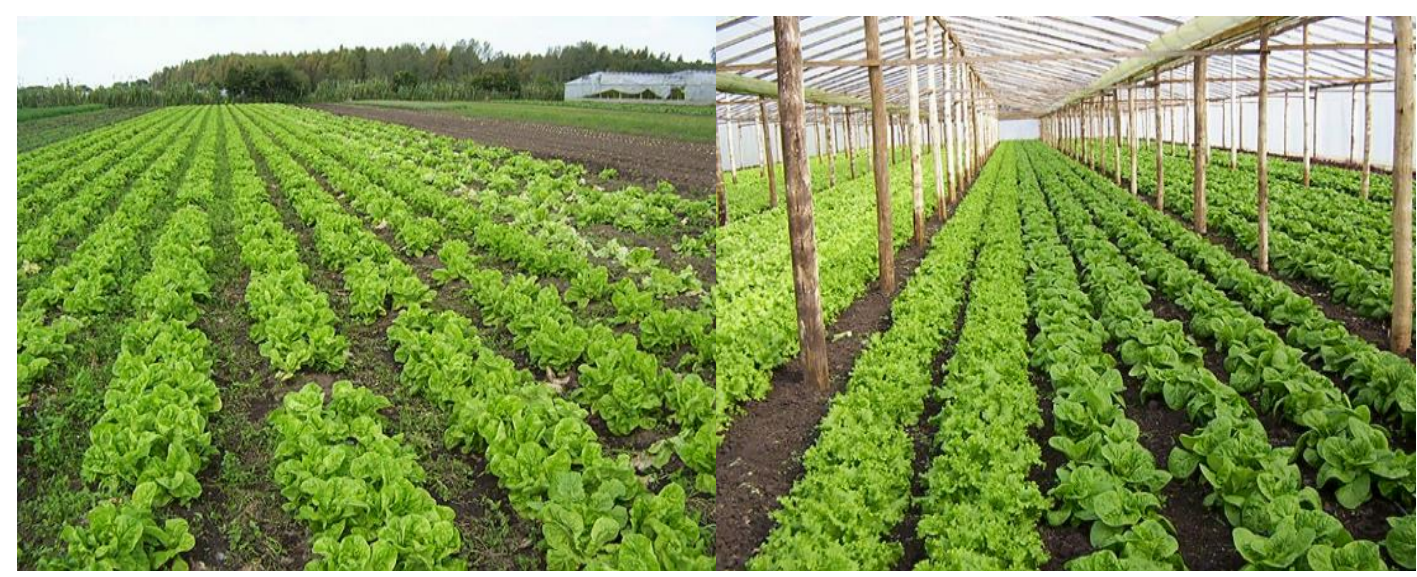

Figura 2.5. Producción de lechuga a campo (izquierda) y en invernáculo (derecha).

En la zona pueden reconocerse tres formas de manejo que "a priori" pueden definirse, de manera general como: 1- Convencional Intensivo: fincas altamente tecnificadas, muchas veces con una amplia superficie ocupada por pocos cultivos de gran valor comercial, un uso intensivo de la tierra y un alto uso insumos químicos (fertilizantes y herbicidas). (Figura 2.6), 2- Convencional de Bajos Insumos: baja tecnificación, fincas de menor superficie, un uso intensivo de la tierra y limitado uso de insumos químicos, 3Orgánicos: fincas caracterizadas por la ausencia de productos químicos y el control manual de la vegetación espontánea.

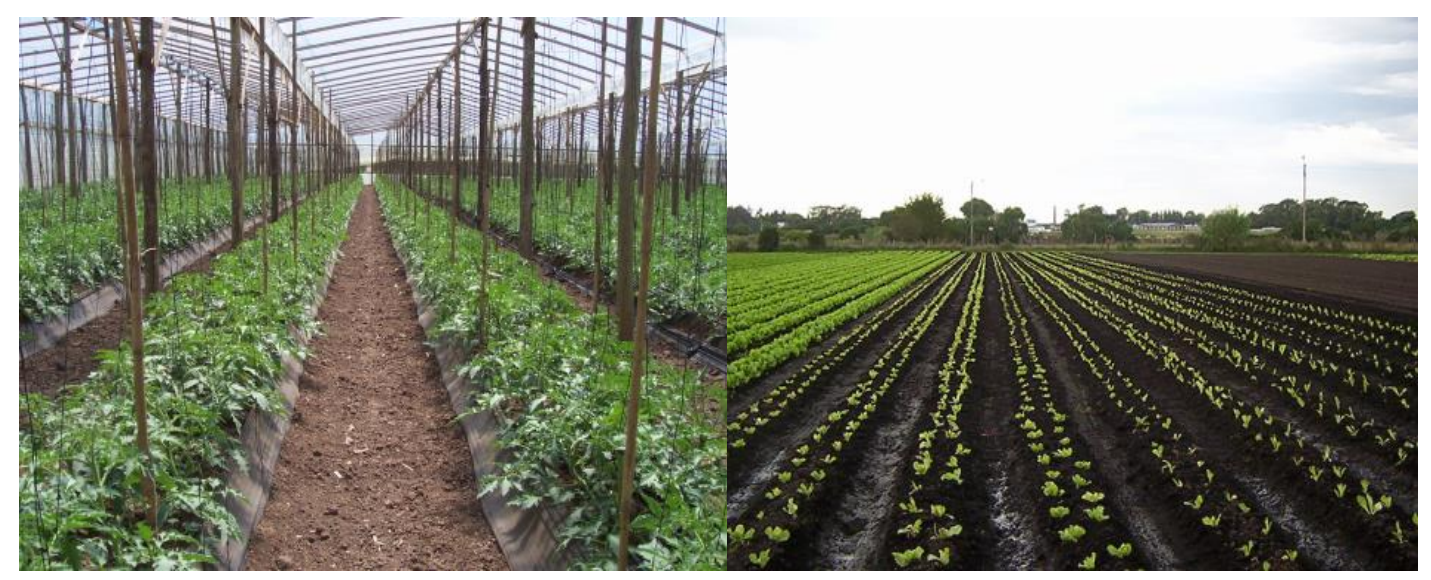

a)

Diversidad Vegetal Espontánea en Agroecosistemas Hortícolas de La Plata y su relación con diferentes estilos de Agricultura: Importancia para la sustentabilidad 


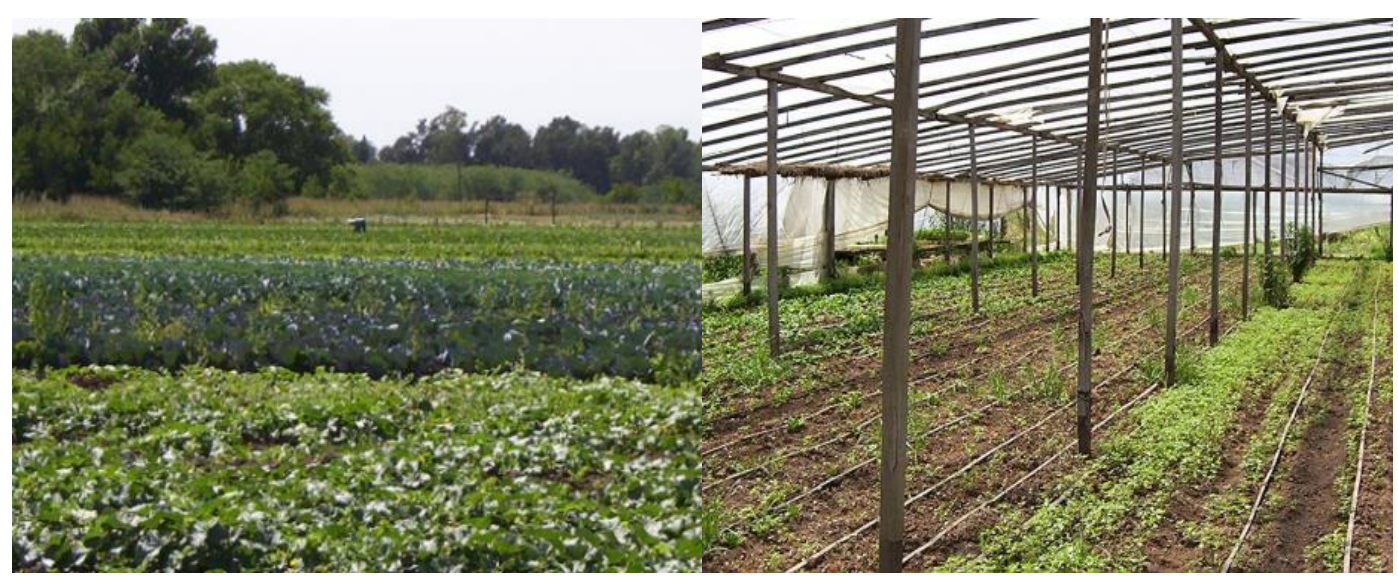

b)

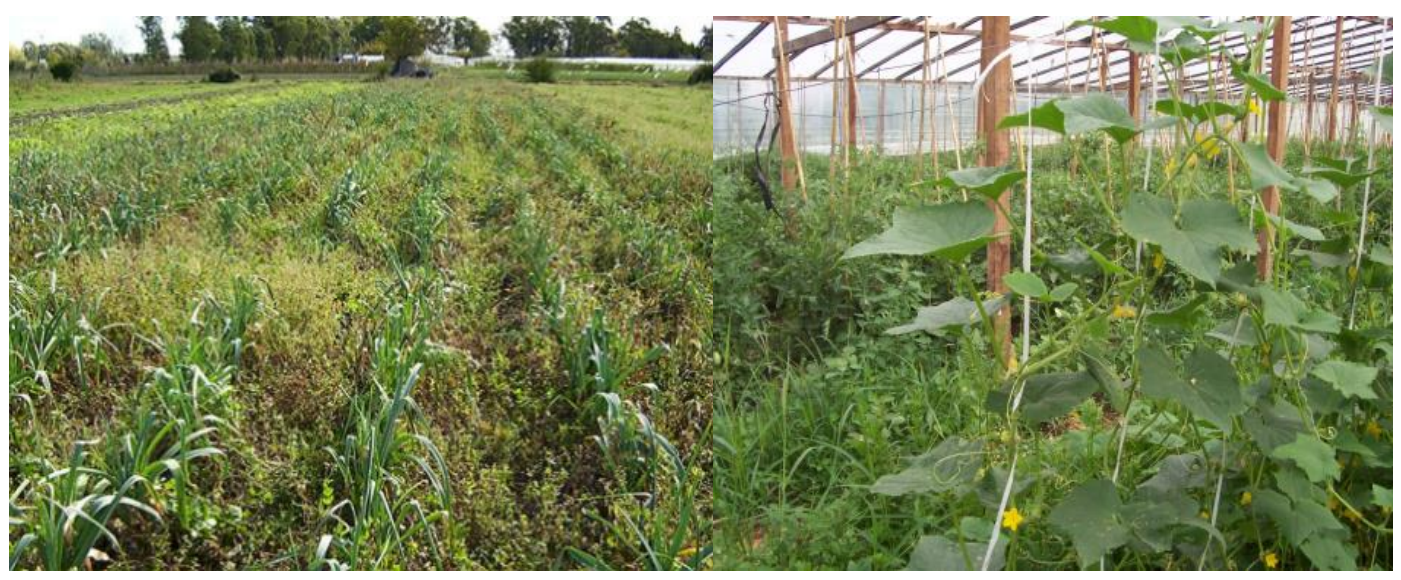

C)

Figura 2.6. Fincas de producción en invernáculo (izquierda) y a campo (derecha) bajo diferente manejo: (a) convencional intensivo, (b) convencional de bajos insumos y (c) orgánico.

Estos estilos de agricultura difieren en sus características según las distintas prácticas utilizadas como, los fertilizantes, el tipo riego, el uso de herbicidas, el empleo de rotaciones, etc. Estas prácticas van acompañadas de una lógica particular de cada agricultor para entender y llevar a cabo la producción. La caracterización de estos estilos de agricultura en base al nivel de intensidad de manejo se analiza en el Capítulo 4 de la tesis. 


\subsection{BIBLIOGRAFÍA}

Archenti A, Ringuelet R \& Salva MC. 1993. Los procesos de diferenciación de los productores hortícolas de La Plata. Etnia No 38/39.

Barsky A. 2005. El periurbano productivo, un espacio en constante transformación. Introducción al estado del debate, con referencias al caso de Buenos Aires. Scripta Nova cripta Nova, Revista Electrónica de Geografía y Ciencias Sociales 9(194): 36. Disponible en: http://www.ub.es/geocrit/sn/sn-194-36.htm.

Barsky A. 2014. Gestionando la diversidad del territorio periurbano desde la complejidad de las instituciones estatales. Implementación de políticas públicas para el sostenimiento de la agricultura en los bordes de la Región Metropolitana de Buenos Aires (2000-2013) (Tesis Doctoral), Universidad Autónoma de Barcelona, España. Disponible en: http://ddd.uab.cat/record/118629.

Benencia R, Cattáneo CA \& Fernández R. 1992. Consecuencias de un proceso de adopción tecnológica reciente: cultivos hortícolas bajo invernáculo en el cinturón verde de Buenos Aires. Centro de Estudios e Investigaciones Laborales CEIL, Buenos Aires. Documento de Trabajo No 31.

Benencia R. 1994. La horticultura Bonaerense: lógicas productivas y cambios en el mercado de trabajo. Desarrollo Económico 34(133): 53-73.

Benencia R \& Souza Casadinho J. 2009a. Introducción. En: Benencia R, Quaranta Q \& Souza Casadinho J (Coord.). Cinturón Hortícola de la Ciudad de Buenos Aires. Cambios sociales y productivos. Ediciones Ciccus, Buenos Aires. 1: 11-35.

Benencia R, Quaranta G \& Tassara C. 2009b. Estructura social agraria, producción y tecnología en el cinturón hortícola de la ciudad de Buenos Aires. En: Benencia R, Quaranta Q \& Souza Casadinho J (Coord.). Cinturón hortícola de la Ciudad de Buenos Aires. Cambios sociales y productivos. Ediciones Ciccus, Buenos Aires. 2: 39-70.

Bozzano H. 2002. El cinturón verde platense: sistema de objetos, sistemas de acciones. Foro CIVEBA Cinturón Verde Metropolitano Buenos Aires. Jornadas AADER de Argentina y el Mercosur, Facultad de Agronomía, UNLP.

Censo Hortiflorícola de Buenos Aires 2005 (CHFBA'05). 2006. Ministerio de Asuntos Agrarios y Ministerio de Economía de la Provincia de Buenos Aires. $115 \mathrm{p}$.

Cieza R. 2004. Asesoramiento Profesional y manejo de nuevas tecnologías en unidades de producción hortícolas del Gran La Plata, Argentina. Scientia agraria 5(1-2): 79-85.

Cieza R, Ferrraris G, Seibane C, Larrañiaga G \& Medicino L. 2015. Aportes a la caracterización de la agricultura familiar en el partido de La Plata. Revista de la Facultad de Agronomía 114(1): 129-142.

Cuellas MV. 2017. Horticultura periurbana. Análisis de la fertilidad de los suelos en invernaderos. Chilean Journal of Agricultural and Animal Sciences 33(2): 163-173.

Diversidad Vegetal Espontánea en Agroecosistemas Hortícolas de La Plata y su relación con diferentes estilos de Agricultura: Importancia 
Garat JJ, Ahumada A, Otero J, Terminiello L, Bello G \& Ciampagna ML. 2009. Las hortalizas típicas locales en el cinturón verde de La Plata: su localización, preservación y valorización. Horticultura Argentina 28(66): 32-39.

Garcia M. 2010. Inicios, consolidación y diferenciación de la horticultura platense. En Svetliza de Nemirovky A (Coord.), Globalización y agricultura periurbana en la Argentina. Escenarios, recorridos y problemas. Maestría en Estudios Sociales Agrarios, FLACSO, Buenos Aires, Serie Monografias 1.

Garcia M. 2012. Análisis de las transformaciones de la estructura agraria hortícola platense en los últimos 20 años. El rol de los horticultores bolivianos. Tesis doctoral. La Plata, Facultad de Ciencias Agrarias y Forestales (UNLP).

Hang G, Kebat C, Bravo M, Larrañaga G, Seibane C, Ferraris G, Otaño M \& Blanco V. 2010. Identificación de Sistemas de Producción Hortícola en el Partido de La Plata, Provincia de Bs. As. Revista Bioagro 22(1): 81-86.

Hurtado MA, Giménez JE, Cabral MG, Silva MD, Martinez, OR, Camilión, MC Sanchez CA, Muntz DE, Gebhard J, Forte L, Boff L Crincoli A \& Lucesoli H. 2006. Análisis ambiental del partido de La Plata. Aportes al ordenamiento territorial 124 p. Disponible en: http://sedici.unlp.edu.ar/handle/10915/27046.

Le Gall J \& García M. 2010. Reestructuraciones de las periferias hortícolas de Buenos Aires y modelos espaciales ¿Un archipiélago verde?. EchoGéo 5(11): 1-15. Disponible en: http://echogeo.revues.org/index11539.html. Ultimo acceso: agosto de 2015.

Lemmi S. 2011. Las clases sociales en la horticultura platense. Ejercicio de teorización, historización y análisis empírico. Mundo Agrario 12(23):1-38. Disponible en: http://www.mundoagrario.unlp.edu.ar/article/view/v12n23a16/198.

Ringuelet R. 2009. La complejidad de un campo social periurbano centrado en las zonas rurales de La Plata. Mundo Agrario 9(7):1-25. Disponible en: http://www.redalyc.org/articulo.oa?id=84511268007.

Simonatto S. 2000. Cambio tecnológico en el sector hortícola de La Plata. Período 198595. En Ringuelet $\mathrm{R}$ (Coord.). Espacio tecnológico, población y reproducción social en el sector hortícola de La Plata. FHCE (UNLP) Serie Estudios e Investigación n³9. 


\section{CAPÍTULO 3}

CARACTERIZACIÓN DE LA DIVERSIDAD VEGETAL ESPONTÁNEA PRESENTE EN EL CINTURÓN HORTÍCOLA DE LA PLATA Y SU VALORACIÓN DESDE LA PERCEPCIÓN DE LOS AGRICULTORES

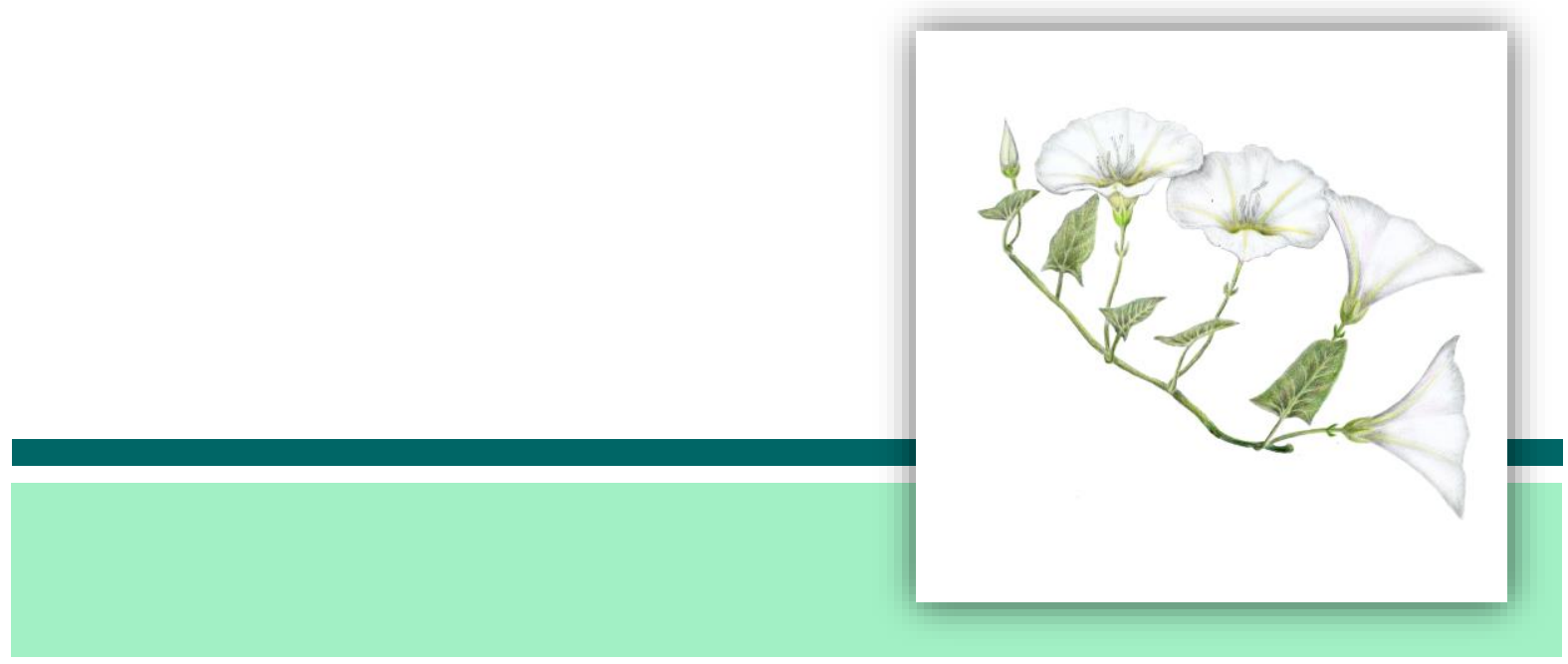




\section{CAPÍTULO 3}

Nosotros desde ya las detestamos, pero algo tienen que tener. La tierra que no da maleza no sirve tampoco para verdura.

Luis Pauletich. Agricultor entrevistado.

\section{CARACTERIZACIÓN DE LA DIVERSIDAD VEGETAL ESPONTÁNEA PRESENTE EN EL CINTURÓN HORTÍCOLA DE LA PLATA Y SU VALORACIÓN DESDE LA PERCEPCIÓN DE LOS AGRICULTORES}

\subsection{INTRODUCCIÓN}

La agricultura sustentable tiene como objetivo desarrollar una producción que sea económicamente viable, socialmente aceptable, suficientemente productiva, que conserve la base de recursos naturales y preserve la integridad del ambiente (Sarandón \& Sarandón, 1993). En este sentido, uno de los desafíos actuales de la agricultura es lograr compatibilizar la producción con el manejo sostenible de la biodiversidad (UNEP, 1997; Tscharntke et al., 2005, 2012; Brussaard et al., 2010; Fisher et al., 2014). Dado que la agricultura es la actividad humana predominante en el mundo, una parte importante de la biodiversidad está presente en los agroecosistemas (Pimentel et al., 1992; Halffer et al., 1999; Foley et al., 2005; FAO, 2013). Durante mucho tiempo, la preocupación por su conservación estuvo centrada en los ecosistemas naturales (Banks, 2004; Clergue et al., 2005; Fischer et al., 2014), siendo más difícil conceptualizarla dentro del contexto de la agricultura (Soini \& Aakkula, 2007; Bátary et al., 2011).

Uno de los componentes fundamentales de la biodiversidad son las plantas que crecen de manera espontánea junto a los cultivos, ya que proveen de servicios ecológicos y recursos biológicos de las plantas de interés agropecuario (Altieri, 1999; Altieri \& Nicholls, 1999; Gliessman, 2001; Swift et al., 2004, de Bello et al., 2010). Representan la base de la diversidad general porque constituyen la fuente de alimento y hábitat para organismos de otros niveles tróficos (Albrecht, 2003; Marshall et al., 2002; 2003). Además, intervienen en

Diversidad Vegetal Espontánea en Agroecosistemas Hortícolas de La Plata y su relación con diferentes estilos de Agricultura: Importancia 
servicios ecosistémicos como, el ciclado de nutrientes, el control de la erosión, la fotosíntesis y la regulación del ciclo del agua, entre otros (Swift et al., 2004; de Bello et al., 2010; Garbach et al., 2014; Gaba et al., 2016). Tradicionalmente, estas plantas han recibido el nombre de "malezas" (del latín malitia que significa malisia) considerándolas organismos indeseables que deben ser eliminados o controlados debido al impacto sobre los rendimientos de los cultivos. Sin embargo, desde el punto de vista ecológico, son especies adaptadas a disturbios recurrentes y disponibilidad de recursos concentrados, lo cual explica su presencia en los agroecosistemas. Los ciclos de cultivo son controlados por el agricultor a través del manejo que realiza, generándose condiciones para el ensamblaje de las comunidades de plantas (Ghersa \& León 1999; Poggio, 2012; Petit et al., 2013). Por lo tanto, las áreas cultivadas presentan una flora particular que puede diferenciarse de la de los ambientes naturales o seminaturales (Harlan, 1976; di Castri \& Youngès; 1996; Martínez-Ghersa et al., 2000), y cuya importancia muchas veces no se ha tenido en cuenta.

El incremento de la diversidad de espontáneas suele asociarse, en parte, con el aumento de la heterogeneidad de hábitats (Benton et al. 2003; Hole et al., 2005; Stupino et al., 2008; Stupino et al. 2014). La heterogeneidad total es el resultado de condiciones abióticas y bióticas originales, y los cambios directos e indirectos resultantes de la intervención del hombre para la instalación y manejo de los cultivos. Este concepto de heterogeneidad ambiental es el que constituye el marco de referencia que se aplica en la tesis, a partir de la cual se hacen los análisis particulares de cada capítulo.

En el país, y en otras regiones del mundo, los estudios sobre las especies acompañantes de los cultivos, se han hecho principalmente en sistemas extensivos (por ejemplo, cultivos de trigo, soja y maíz), que presentan baja diversidad cultivada y de estructura y manejo similar (de la Fuente et al., 1999; Suárez et al., 2001; Poggio et al., 2005; Scursoni et al., 2014). Sin embargo, no se ha tenido en cuenta los sistemas hortícolas (Shepherd et al., 2003). La horticultura difiere del resto de las producciones pampeanas en que se desarrolla en pequeñas áreas, es más diversificada y se localiza generalmente en un ámbito periurbano (Archenti et al., 1993; Barsky, 2005).

En el Cinturón Hortícola Platense las fincas poseen dimensiones pequeñas a medianas (7 ha en promedio). Se producen alrededor de 30 especies cultivadas, en parcelas que no superan la hectárea. Además, se desarrollan diferentes estilos de agricultura, que pueden 
sintetizar en convencionales y orgánicos, diferenciados comúnmente por el uso o no de herbicidas, insecticidas y fertilizantes sintéticos (Ahnström, 2009).

Dada esta heterogeneidad dentro del contexto del periurbano platense, es esperable que el área de estudio presente una importante riqueza de especies, géneros y familias de plantas espontáneas y otras características asociadas (morfología, ciclo de vida, origen y valor utilitario).

La presencia de las plantas espontáneas puede relacionarse, además, con la percepción (entendida como la visión y conocimiento) que tiene el agricultor sobre la biodiversidad. Cada agricultor tiene una experiencia directa con la biodiversidad durante su práctica diaria, por lo tanto, entre los distintos agricultores puede variar la visión que tengan sobre la misma (Fisher \& Young, 2007; Soini \& Aakkula, 2007; Kelemen et al., 2013). Algunos autores encontraron que los agricultores solo valoran y reconocen los organismos que están fuera de la finca. En cambio, dentro de la misma, consideran que las plantas espontáneas son perjudiciales y que es una buena práctica mantener el campo "limpio" de vegetación (Herzon \& Mikk, 2007; Soini \& Akkula, 2007).

Una de las preocupaciones que atraviesa la agricultura convencional es el alto uso de plaguicidas. La agricultura sustentable propone el reemplazo de insumos externos por procesos ecológicos que provee la biodiversidad. En este sentido, el conocimiento que tenga el agricultor influye en el manejo de la biodiversidad y su impacto, ya que el valor que le atribuye determina la acción de controlar o no la vegetación espontánea (Radosevich \& Ghersa, 2007; Wilson et al., 2008; Kelemen et al., 2013). Sin embargo, pocos estudios en otras regiones han analizado la percepción que los agricultores tienen acerca de la vegetación espontánea (Wilson et al., 2008; Herzon et al.; 2009; Stilma et al.; 2009; Kelemer et al., 2013). En diversos trabajos se encontró una variedad de conceptos expresados por los agricultores mayormente relacionados con una valoración negativa de las espontáneas (Mace et al., 2007; Wilson et al., 2008). En la zona de La Plata, es esperable que prevalezca el concepto tradicional de maleza, que se vería reflejado en el predominio de una percepción de las especies espontáneas como algo perjudicial o negativo por parte de los agricultores. Una valoración negativa influye en la permanencia y/o abundancia de especies, a través de una mayor presión de control sobre las mismas.

Diversidad Vegetal Espontánea en Agroecosistemas Hortícolas de La Plata y su relación con diferentes estilos de Agricultura: Importancia 
La caracterización de la flora del Cinturón Hortícola de La Plata, permitirá conocer la riqueza taxonómica de la zona y sus características asociadas. Esto podría establecer el rol funcional de las plantas espontáneas vinculado a otros componentes de la cadena trófica. A su vez, el análisis de la valoración de la vegetación espontánea según la apreciación de los agricultores puede ser indicadora de la posibilidad de permanencia de dichas especies en los agroecosistemas. Estos aspectos contribuyen a entender como potenciar el mantenimiento de la biodiversidad para garantizar los servicios ecológicos que provee en el diseño de sistemas sustentables.

El objetivo de este capítulo es caracterizar la diversidad y composición vegetal espontánea presente en el Cinturón Hortícola Platense y evaluar la percepción que los agricultores tienen acerca de la misma. Para ello, se ponen a prueba las siguientes hipótesis.

Hipótesis 1:

La heterogeneidad de hábitats afecta la diversidad de plantas espontáneas. En el Cinturón Hortícola Platense, debido principalmente a su complejidad de cultivos y manejos, ésto se manifiesta en una significativa diversidad de especies, géneros y familias de espontáneas, grupos funcionales y especies de valor utilitario.

Hipótesis 2:

Independientemente del grado conocimiento que tenga el agricultor acerca de las especies acompañantes prevalece su percepción como malezas, reflejado en una dominante valoración de los aspectos negativos.

\subsection{METODOLOGÍA}

\subsubsection{Selección de las fincas y realización de entrevistas}

Se seleccionaron 32 fincas de producción hortícola comprendidos dentro de la región periurbana del Gran La Plata. Las mismas se situaron en las localidades de Pereyra, Lisandro Olmos, Los Hornos, Arana, El Pato, Colonia Urquiza, Etcheverry y Gorina, de manera de abarcar diferentes zonas del Cinturón Hortícola Platense (Figura 3.1).

Diversidad Vegetal Espontánea en Agroecosistemas Hortícolas de La Plata y su relación con diferentes estilos de Agricultura: Importancia 
En dos casos, la misma finca presentaba sectores manejados por distintos agricultores, con lo cual se consideró individualmente como finca cada área manejada por una sola persona. La selección y localización de las fincas se realizó a través de la consulta a profesionales de la Ciencias Agrarias (UNLP), técnicos y agricultores. Se visitaron las fincas y, sobre la base de las características de las mismas, se seleccionó un grupo abarcativo de situaciones de manejo convencionales y orgánicos. Los sistemas seleccionados comprendieron formas de cultivo en invernáculo (bajo cubierta), a campo (aire libre), o ambas formas de manejo (mixtos).

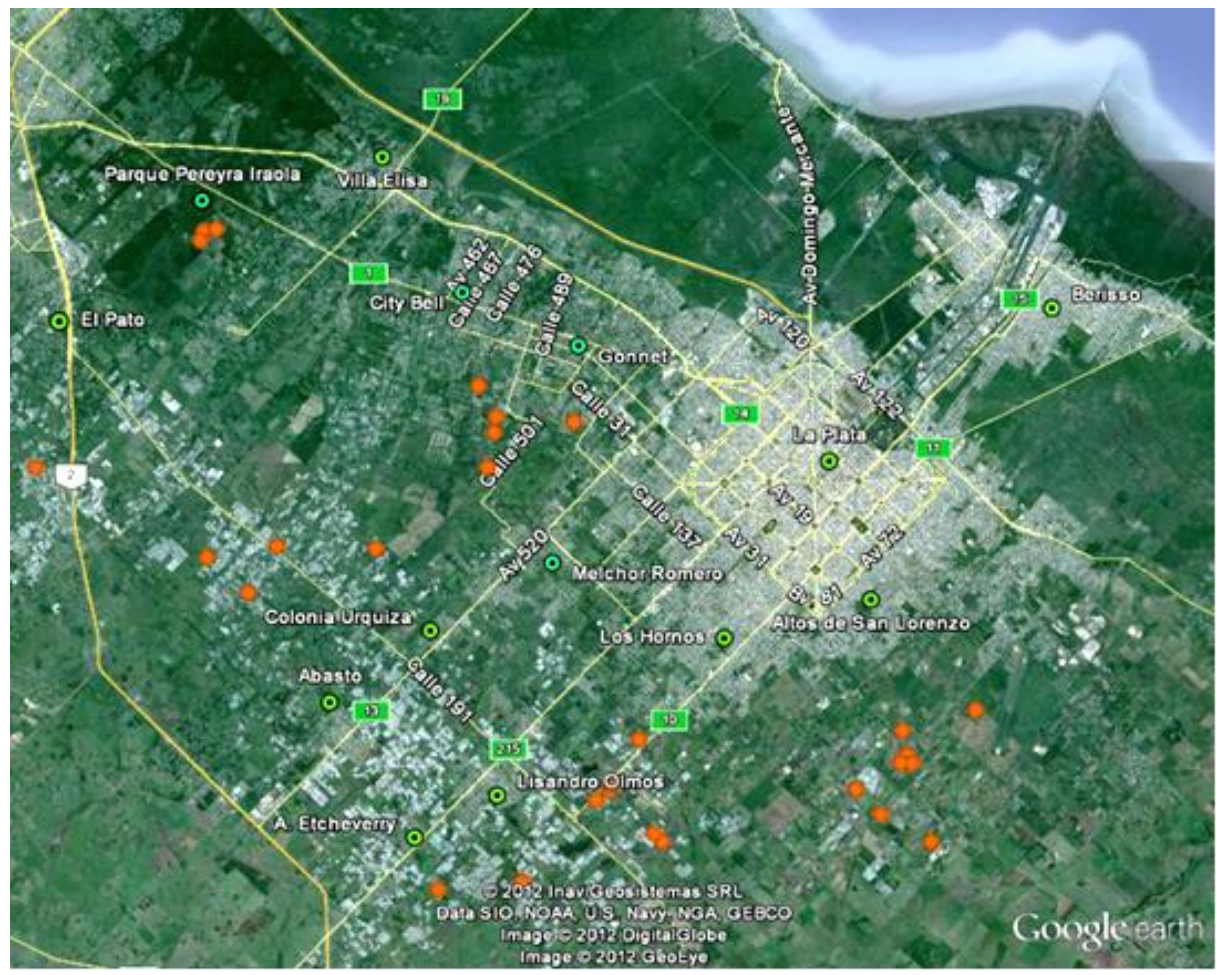

Figura 3.1. Distribución de las unidades de muestreo en el Cinturón Hortícola. Los círculos rojos indican las localizaciones geográficas de las fincas relevadas, los cuadrados verdes indican rutas provinciales y, los puntos verdes y negros las localidades citadas en el texto. Fuente: imagen de Google Earth (2017). Modificado.

\subsubsection{Muestreo de la vegetación espontánea}

El muestreo se realizó dentro de la superficie cultivada de cada establecimiento. En cada finca se recorrió el área y se identificaron dos tipos de unidades espaciales de acuerdo 
al criterio de uso de la tierra y/o el cultivo dominante: 1) Parcelas cultivadas: unidades homogéneas definidas por la presencia de uno o más cultivos dominantes. En este último caso el agricultor los considera como una unidad porque tienen un manejo o ciclo de vida similar (Figura 3.2), 2) Parcelas no cultivadas: parcelas dominadas por vegetación espontánea que pueden contener o no residuos de cultivos en post-cosecha.

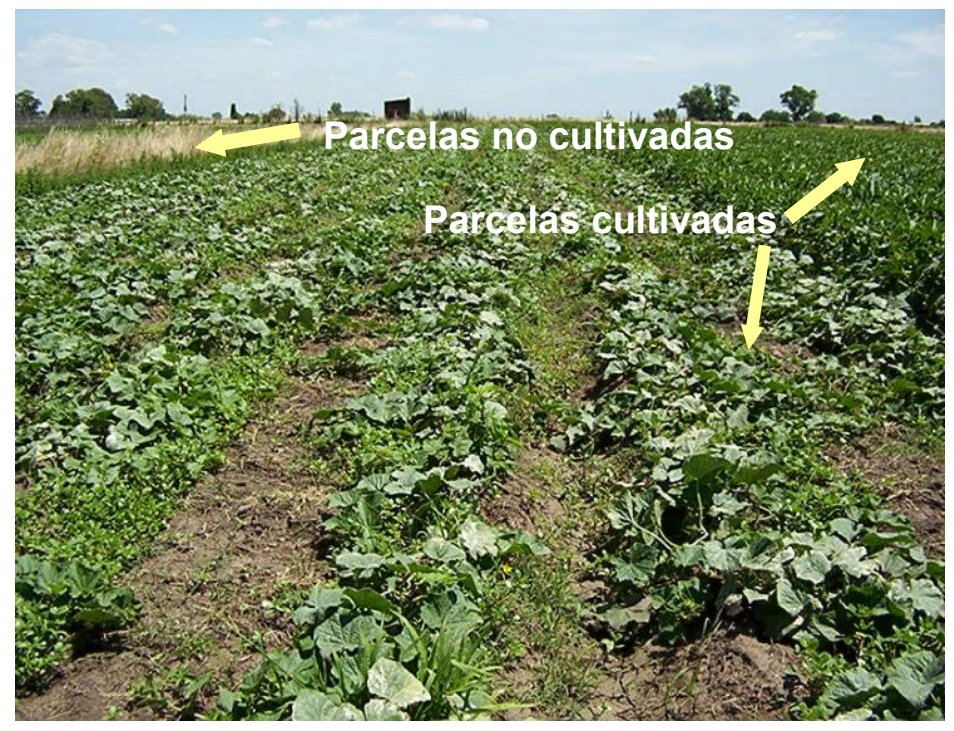

Figura 3.2. Unidades espaciales identificadas: parcelas cultivadas y no cultivadas.

El muestreo se realizó sólo en las parcelas cultivadas. Para establecer la superficie adecuada de cada unidad de muestreo, se realizaron muestreos preliminares en dichas unidades de vegetación utilizando el método del área mínima (Mueller-Dombois \& Ellenberg, 1974). Este método se basa en ir duplicando el área muestreada en un sitio homogéneo (un determinado cultivo), e ir registrando, para cada superficie sucesiva relevada, el número de especies acumulada hasta que no se registren nuevas especies (Mateucci \& Colma, 1982). Habiendo considerado diversos cultivos, el área mínima mayor obtenida no superó los $16 \mathrm{~m}^{2}$ (Stupino et al., 2008). Considerando esto, se utilizó en todas las fincas una unidad de muestreo (quadrat) estándar de $25 \mathrm{~m}^{2}$. El tamaño empleado fue superior a lo obtenido en los muestreos preliminares para que el área de cada unidad sea lo suficientemente grande como para detectar diferencias en la riqueza de plantas entre distintos sistemas de manejo (Manhoudt et al., 2005). Establecer un área de muestreo estándar permitió, además, que la composición de especies acompañantes en distintos tipos de cultivos pueda compararse sin estar afectada por las dimensiones de la unidad de muestreo. 
El muestreo se realizó durante el período más favorable para el crecimiento de las especies acompañantes (fin de primavera e inicio del verano) y en condiciones de buen desarrollo del cultivo. Se instaló, en cada parcela de cultivo, una línea transecta de $25 \mathrm{~m}$ que constituye el eje mayor de una unidad de muestreo rectangular de $25 \mathrm{~m}^{2}$ (medio metro a cada lado de la línea por $25 \mathrm{~m}$ de largo) cuya área es adecuada para el muestreo de espontáneas (Figura 3.3) (Mueller-Dombois \& Ellenberg, 1974). En el caso de los cultivos cuya superficie total en la finca no superó la hectárea, el muestreo se realizó en la parcela mayor de cada cultivo que cumplía con la condición de buen desarrollo del cultivo (descartando el estado de plántula o en senescencia) en una unidad de muestreo por cultivo diferente. Para el caso en que se superó dicha superficie, se tomó un número adicional de unidades muestrales que fuese proporcional a la superficie total del área cultivada a razón de una unidad muestral por hectárea. Incrementar el número de unidades de muestreo en función de la superficie de cada parche de cada cultivo, permite comparar la riqueza a nivel de cultivo y de finca habiendo efectuado una ponderación del efecto de área de la parcela de cultivo y la finca. En cada unidad se registraron las especies presentes.

Se realizó un esquema de la distribución de las parcelas y cultivos de la finca. Además, se registraron las especies cultivadas y el número de variedades por cultivo (Figura 3.4). 


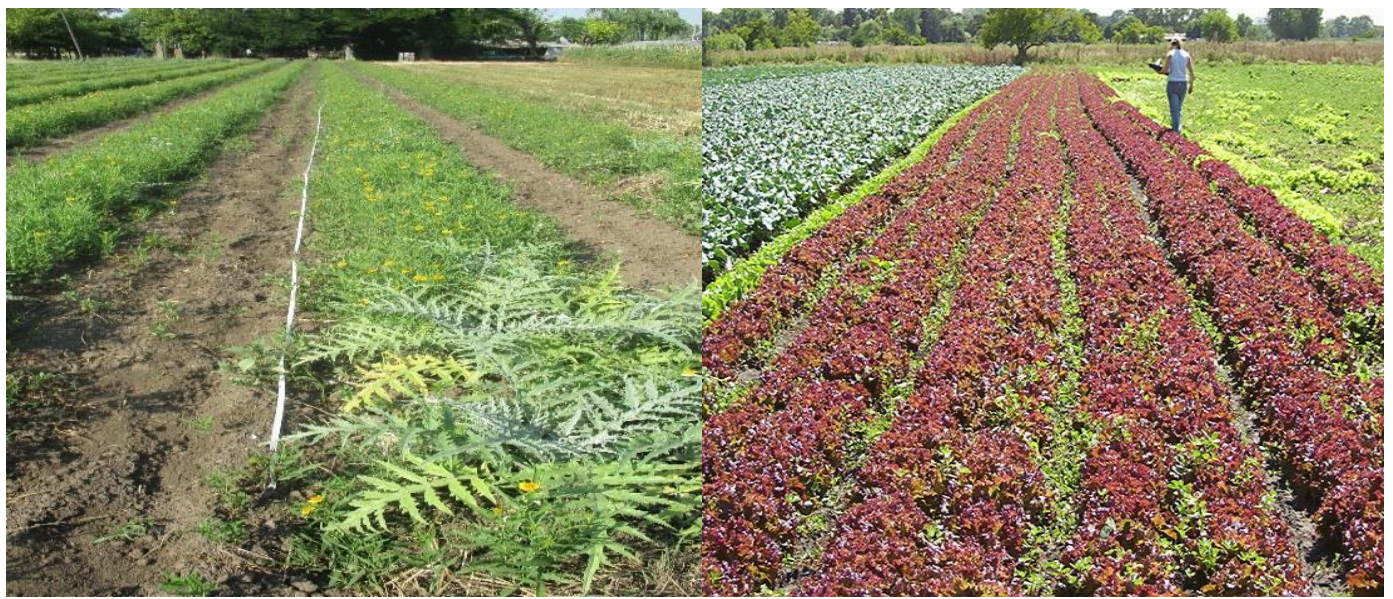

a)

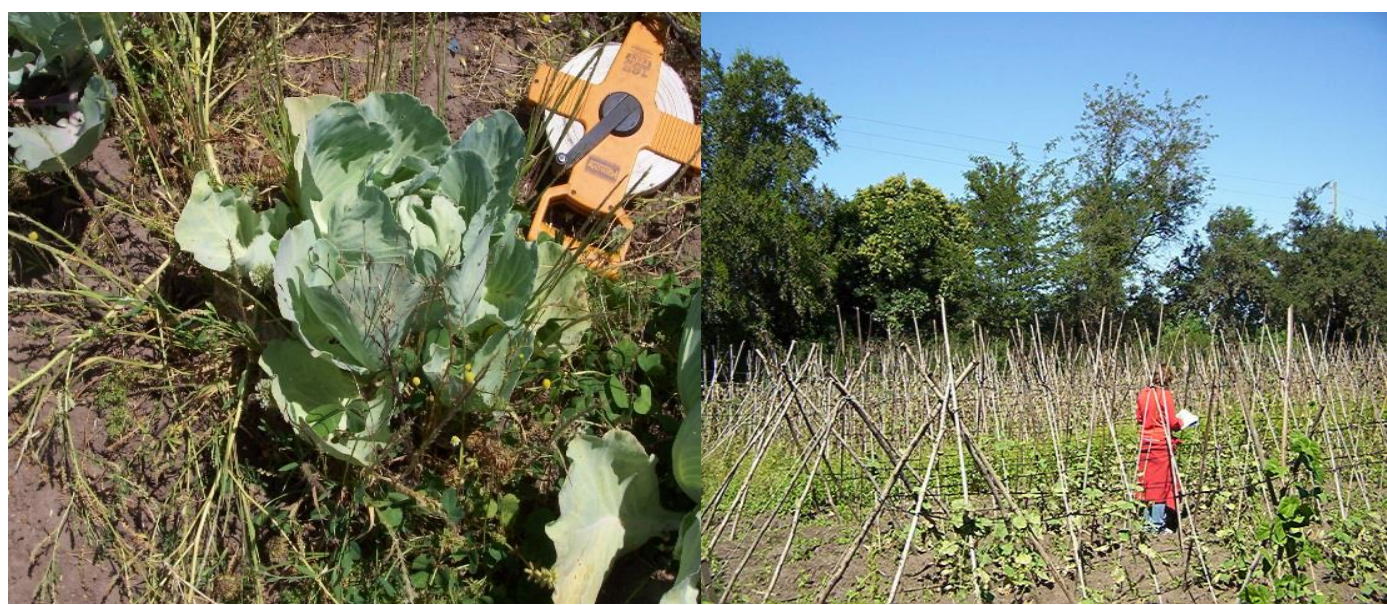

b)

Figura 3.3. a) Línea de transecta de $25 \mathrm{~m}$, definida a partir de una cinta métrica ubicada a lo largo del cultivo para establecer la unidad de muestreo $\left(25 \mathrm{~m}^{2}\right)$. b) Etapas del muestreo. Fuente: fotografías de la autora. 


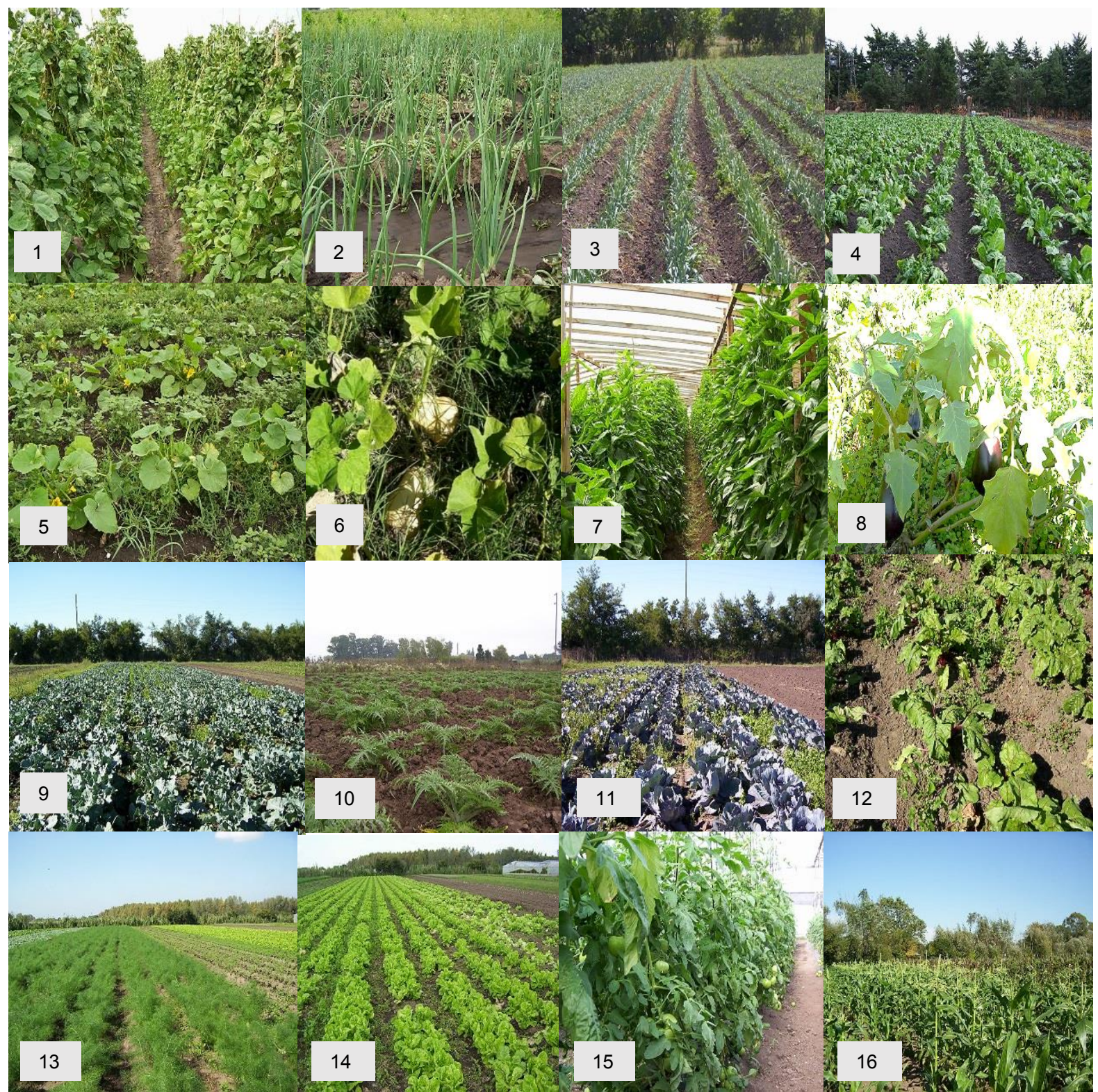

Figura 3.4. Fotografías de algunos cultivos relevados: (1) chaucha, (2) verdeo, (3) puerro, (4) acelga, (5) zapallito de tronco, (6) zapallo anco, (7) morrón, (8) berenjena, (9) brócoli, (10) alcaucil (11) repollo, (12) remolacha, (13) hinojo, (14) lechuga, (15) tomate, (16) maíz. Fuente: fotografías de la autora.

\subsubsection{Determinación de plantas y clasificación de grupos funcionales}

En base a la consulta bibliográfica (Cabrera, 1963-70; Marzocca, 1976; Cabrera \& Zardini, 1978; Molina 1998, 1999), el empleo de lupa binocular, consulta personal y el apoyo del 
material de referencia del Herbario del Museo de Ciencias Naturales de La Plata se determinaron los ejemplares coleccionados. La nomenclatura se actualizó de acuerdo al Catálogo on-line de Plantas del Instituto de Botánica Darwinion (última revisión en noviembre de 2012). Para establecer la importancia de las especies, se calculó el valor de constancia de cada una, es decir, la proporción en que cada especie está presente en el conjunto total de las fincas.

Se clasificaron y cuantificaron las especies en grupos funcionales según el ciclo de vida, morfología y origen ${ }^{1}$. De acuerdo con el ciclo de vida se consideraron las especies anuales, bianuales y perennes. Para contabilizar cada categoría se consideró el mayor período de vida en que las especies presentan más de un ciclo (por ejemplo, una especie anual-perenne se contabilizó como perenne). La morfología se dividió en dicotiledóneas y monocotiledóneas (familias Poaceae y Cyperaceae). De acuerdo con el origen se clasificaron en exóticas, nativas y cosmopolitas. Se registró el valor utilitario de las mismas (medicinal, comestible, etc.) a partir de la consulta de diferentes fuentes bibliográficas (Marzocca 1976, 1997; CONABIO 2006-2012; Rapoport et al., 2009; Hurrell et al., 2009; Hernández \& Arambarri, 2011).

${ }^{1}$ En esta tesis se utiliza la expresión "grupos funcionales", que según algunos autores que han trabajado en temas afines (de La Fuente et al. (2006) y Poggio (2007) reúne las características asociadas de las especies estudiadas según el origen, ciclo de vida y morfología.

Se ha seguido ese criterio con el fin de facilitar las comparaciones bibliográficas, pero sin perder el tratamiento particular de cada componente. No obstante, se reconocen algunos problemas de la mencionada composición del término "grupos funcionales"; por ejemplo, claramente, el lugar de origen de las especies no es una propiedad funcional.

\subsubsection{Evaluación de la percepción de los agricultores sobre las plantas espontáneas}

A partir de entrevistas previas con los agricultores se elaboró una encuesta focalizada (Ander-Egg, 1985), que consiste en armar una lista de preguntas que indican los puntos sobre los que debe basar la entrevista, pero no señala ni el orden ni el volumen de las respuestas, que quedan a libertad del entrevistado. Se entrevistó a los agricultores varones con el soporte de un grabador y se tomó nota en una encuesta en papel. Se relevaron aspectos vinculados a la valoración de las especies espontáneas por los agricultores. Como aspectos positivos de la vegetación espontánea se consideraron: el aporte de materia orgánica y de nutrientes; el alberge de insectos benéficos; la protección de los cultivos contra el sol o las heladas; la protección del suelo contra pérdida de agua y erosión. Los

Diversidad Vegetal Espontánea en Agroecosistemas Hortícolas de La Plata y su relación con diferentes estilos de Agricultura: Importancia 
aspectos negativos considerados fueron: la dificultad en la práctica de labranza y/o la cosecha; la quita de nutrientes al cultivo y albergue de plagas. Dentro de cada aspecto, positivo o negativo de la vegetación espontánea, se registró si lo consideraban importante; lo conocían; no opinaban o desconocían; o no lo consideraban un beneficio, respectivamente. Se registró además si a los agricultores "les molestaban" las espontáneas y el posible valor de uso que le otorgaban (medicinal, comestible, etc.).

\subsection{RESULTADOS}

\subsubsection{Diversidad taxonómica}

Se determinaron 152 especies, 120 géneros y 40 familias de espontáneas; estos números serían asimismo subestimaciones ya que un $16 \%$ del material no pudo ser determinado (Tabla 3.1). La determinación de los restantes ejemplares se dificultó debido al elevado número de plántulas en general y a la presencia de gramíneas y compuestas que no presentaron estructuras reproductivas. Las familias que estuvieron más representadas en orden de importancia fueron: Asteraceae $(17,2 \%)$, Poaceae $(11,2 \%)$ y Solanaceae (8,6\%). Las siguientes 5 familias fueron Brassicaceae, Fabaceae, Apiaceae, Amaranthaceae y Malvaceae, que representaron, sumadas, el 26,5\% del total de especies.

Se relevaron en total de 40 especies de cultivos, algunas de las cuales se encontraron también de manera espontánea en las parcelas: acelga (Beta vulgaris var cicla $\mathrm{L}$ ), achicoria (Cichorium intybus L.), ají (Capsicum annum L.), ajo (Allium sativum L.), albahaca (Ocinum basilicum L.), alcaucil (Cynara cardunculus L. var. Scolymus), apio (Apium graveolens L.), arveja (Pisum sativum L.), batata (Ipomoea batatas (L.) Poir), berenjena (Solanum melongena L.), brócoli (Brassica oleracea L. var. botrytis D.C.), cebolla (Allium cepa L.), chaucha (Phaseolus vulgaris L.), ciboulette (Allium schoenoprasum L.), coliflor (Brassica oleracea L. var. botrytis D.C.), espinaca (Spinacia oleracea L.), frutilla ( Fragaria vesca L. ), haba (Vicia faba ), hinojo (Foeniculum vulgare Mill.), lechuga (Lactuca sativa L.), maíz (Zea maiz L.), melón (Cucumis melo L.), nabo (Brassica napus L.), orégano (Origanum vulgare L.), papa (Solanum tuberusum L.), pepino (Cucumis sativus L.), perejil (Petroselinum crispum (Mill.)Nym.), poroto (Vigna sinensis (L.)), puerro (Allium porrum L.), rabanito ( Rapanus satinus L.), radicheta (Cichorium intybus L.), remolacha (Beta vulgaris L.var rapacea), repollito de Bruselas (Brasicca oleracea L var. Gemmifera Zank.), repollo

Diversidad Vegetal Espontánea en Agroecosistemas Hortícolas de La Plata y su relación con diferentes estilos de Agricultura: Importancia 
(Brassica oleracea), rúcula (Eruca sativa Mill.), tomate (Lycopersicon esculetum Mill.), verdeos (Allium cepa L.), zanahoria (Daucus carota L.), zapallito (Cucurbita maxima var. zapallito (Carr.) y zapallo anco (Cucurbita mostacha Duch.).

Tabla 3.1. Especies, géneros y familias determinadas en el Cinturón Hortícola y sus características funcionales según origen, Or (E: exótica, N: nativa, C: cosmopolita), ciclo de vida, Cv (A: anuales, B: biennal, P: perenne); morfología, Mo (M: monocotiledóneas, D: dicotiledóneas) y valor utilitario, Val (For: forrajera, Med: medicinal, Com: comestible, Orn: ornamental, Ces: césped, Mad: maderero). Se indica además la Constancia de las especies (Co), que representa, la proporción de fincas en la que una especie está presente (\%).

\begin{tabular}{|c|c|c|c|c|c|c|c|}
\hline Nombre científico & Nombre vulgar & Familia & Or & Cv & Mo & Val & Co \\
\hline Acicarpha procumbens Less. & & Calyceraceae & $\mathrm{N}$ & $\mathrm{P}$ & D & For & 3 \\
\hline Acicarpha tribuloides Juss. & Cardo torito, roseta & Calyceraceae & N & A & $\mathrm{D}$ & Med & 9 \\
\hline Adiantum raddiatum C. Presl & Culandrillo & Pteridaceae & $\mathrm{N}$ & $\mathrm{P}$ & $\left({ }^{*}\right)$ & Med & 3 \\
\hline Allium fistulosum $\mathrm{L}$. & Verdeo & Liliaceae & E & $\mathrm{P}$ & $\mathrm{D}$ & Com & 3 \\
\hline Allium porrum $\mathrm{L}$. & Puerro & Liliaceae & $\mathrm{E}$ & $\mathrm{P}$ & $\mathrm{D}$ & Com-Med-Orn & 3 \\
\hline $\begin{array}{l}\text { Alternanthera philoxeroides (Mart.) } \\
\text { Griseb. }\end{array}$ & Lagunilla & Poaceae & N & B-P & M & Med & 53 \\
\hline $\begin{array}{l}\text { Amaranthus crispus (Lesp. \& } \\
\text { Thévenau) A. Terracc. }\end{array}$ & Lengua de vaca & Amaranthaceae & N & A & $\mathrm{D}$ & Med & 3 \\
\hline Amaranthus hybridus L. hybridus & Yuyo colorado & Amaranthaceae & N & A & $\mathrm{D}$ & For & 87 \\
\hline Amaranthus lividus $\mathrm{L}$. & Ataco & Amaranthaceae & N & A & $\mathrm{D}$ & Com & 47 \\
\hline Amaranthus viridis $\mathrm{L}$. & Bledo & Amaranthaceae & $\mathrm{N}$ & A-P & D & Com-For-Med & 9 \\
\hline Ambrosia tenuifolia Spreng & $\begin{array}{l}\text { Altamisa, ajenjo de } \\
\text { campo }\end{array}$ & Asteraceae & $\mathrm{E}$ & $P$ & $\mathrm{D}$ & Med & 9 \\
\hline Ammi majus L. & $\begin{array}{l}\text { Apio cimarrón, cicuta } \\
\text { negra }\end{array}$ & Apiaceae & E & A & D & Med & 28 \\
\hline Anagallis arvensis $\mathrm{L}$. & $\begin{array}{l}\text { Anagalis, no me } \\
\text { olvides }\end{array}$ & Primulaceae & $\mathrm{E}$ & A & $\mathrm{D}$ & For-Med-Orn & 19 \\
\hline Anoda cristata (L.) Schlecht & Malva, oreja de gato & Malvaceae & $\mathrm{N}$ & A & $\mathrm{D}$ & $\begin{array}{l}\text { Com- For-Me- } \\
\text { Orn }\end{array}$ & 34 \\
\hline Anthemis cotula L. & Manzanilla cimarrona & Asteraceae & $\mathrm{E}$ & A & $\mathrm{D}$ & Med & 6 \\
\hline Arctium minus (Hill) Bernh & Vardana & Asteraceae & E & B & $\mathrm{D}$ & Med & 3 \\
\hline Artemisia verlotiorum Lamotte & Ajenjo & Asteraceae & $\mathrm{E}$ & $\mathrm{P}$ & $\mathrm{D}$ & Med & 3 \\
\hline Baccharis trimera (Less.) DC. & Carqueja & Asteraceae & N & $\mathrm{P}$ & $\mathrm{D}$ & Med & 6 \\
\hline Beta vulgaris $\mathrm{L}$. & Remolacha & Chenopodiaceae & E & B & $\mathrm{D}$ & Com-Med & 3 \\
\hline Beta vulgaris $\mathrm{L}$. var cicla $\mathrm{L}$. & Acelga & Chenopodiaceae & $\mathrm{E}$ & $\mathrm{P}$ & D & Com & 9 \\
\hline Bidens subalternans DC. & Amor seco & Asteraceae & $\mathrm{N}$ & A & D & Med & 3 \\
\hline Bowlesia incana Ruiz \& Pav. & Perejilillo & Apiaceae & N & A & $\mathrm{D}$ & & 21 \\
\hline $\begin{array}{l}\text { Brassica oleracea L. var Capitata } \\
\text { L. }\end{array}$ & Repollo & Brassicaceae & E & $A-B$ & $\mathrm{D}$ & Com & 3 \\
\hline Brassica rapa L. & Nabo & Brassicaceae & $\mathrm{E}$ & A-B & D & Com & 6 \\
\hline
\end{tabular}


Tabla 3.1. (Continúa).

\begin{tabular}{|c|c|c|c|c|c|c|c|}
\hline Nombre científico & Nombre vulgar & Familia & Or & Cv & Mo & Val & Co \\
\hline Bromus catharticus Vahl & $\begin{array}{l}\text { Cebadilla criolla, } \\
\text { cebadilla }\end{array}$ & Poaceae & $\mathrm{N}$ & B-P & M & For-Med & 12 \\
\hline Capsella bursa-pastori (L.) Medik & Bolsa de pastor & Brassicaceae & $E$ & $A-B$ & $\mathrm{D}$ & For-Med & 62 \\
\hline Capsicum annum L. & Pimiento & Solanaceae & $\mathrm{E}$ & A & $\mathrm{D}$ & Com-Med & 3 \\
\hline Cardamine bonariensis Pers. & Berro silvestre & Brassicaceae & $\mathrm{N}$ & $\mathrm{P}$ & $\mathrm{D}$ & Com & 9 \\
\hline Carduus acanthoides L. & $\begin{array}{l}\text { Cardo negro, cardo } \\
\text { chileno }\end{array}$ & Asteraceae & $\mathrm{E}$ & A & $\mathrm{D}$ & Med & 44 \\
\hline Casuarina cunninghamiana Miq. & Casuarina & Casuarinaceae & $\mathrm{E}$ & $\mathrm{P}$ & $\mathrm{D}$ & Med-Orn & 6 \\
\hline $\begin{array}{l}\text { Centaurium pulchellum (Sw.) } \\
\text { Druce }\end{array}$ & Yuyo amargón & Gentianaceae & $\mathrm{E}$ & $A$ & $\mathrm{D}$ & Med & 3 \\
\hline Chenopodium album $\mathrm{L}$. & Quinua blanca & Chenopodiaceae & $E$ & $A$ & $\mathrm{D}$ & Com-For-Med & 69 \\
\hline Chenopodium ambrosioides $\mathrm{L}$. & Paico & Chenopodiaceae & $\mathrm{N}$ & $\mathrm{P}$ & $\mathrm{D}$ & Com-Med & 3 \\
\hline Cichorium intybus $\mathrm{L}$. & Radicheta & Asteraceae & $E$ & $A-B$ & $\mathrm{D}$ & Com-Med & 40 \\
\hline Cirsium vulgare (Savi) Ten. & Cardo negro & Asteraceae & $\mathrm{E}$ & $A-B$ & $\mathrm{D}$ & Com-Med-Mel & 25 \\
\hline Conium maculatum $\mathrm{L}$. & Cicuta & Apiaceae & $E$ & $A-B$ & $\mathrm{D}$ & Med & 12 \\
\hline Convolvulus arvensis $\mathrm{L}$. & Campanilla correhuela & Convolvulaceae & $\mathrm{E}$ & $P$ & $\mathrm{D}$ & Med & 69 \\
\hline Conyza bonariensis (L.) Cronquist & $\begin{array}{l}\text { Rama negra, yerba } \\
\text { carnicera }\end{array}$ & Asteraceae & $\mathrm{N}$ & $A$ & $\mathrm{D}$ & Med & 34 \\
\hline Coriandrum sativum $\mathrm{L}$. & Coriandro & Apiaceae & $E$ & $A$ & $\mathrm{D}$ & Med-Com & 3 \\
\hline $\begin{array}{l}\text { Cucurbita maxima Duchesne ssp. } \\
\text { Andreana }\end{array}$ & Zapallito amargo & Cucurbitaceae & $\mathrm{N}$ & A & $\mathrm{D}$ & Com-Med & 19 \\
\hline Cuscuta indecora Choisy & Cuscuta & Convolvulaceae & $\mathrm{E}$ & A & $\mathrm{D}$ & & 3 \\
\hline $\begin{array}{l}\text { Cyclospermum leptophyllum } \\
\text { (Pers.) Sprague }\end{array}$ & $\begin{array}{l}\text { Apio silvestre, } \\
\text { culandrillo }\end{array}$ & Apiaceae & $\mathrm{N}$ & $A$ & $\mathrm{D}$ & Med-For & 31 \\
\hline Cynodon dactylon L (Pers.) & $\begin{array}{l}\text { Gramón, pata de } \\
\text { perdiz }\end{array}$ & Poaceae & $\mathrm{N}$ & $A$ & M & For-Ces-Med & 75 \\
\hline Cyperus sp. & & Cyperaceae & & & M & & 22 \\
\hline Cyperus rotundus L. & Cebollín & Cyperaceae & $\mathrm{N}$ & $\mathrm{P}$ & $\mathrm{D}$ & Com-Med & 31 \\
\hline Cyperus surinamensis Rottb. & Coyolillo & Cyperaceae & $\mathrm{N}$ & A-P & M & & 3 \\
\hline Datura ferox $\mathrm{L}$. & Chamico & Solanaceae & $\mathrm{N}$ & $A$ & $\mathrm{D}$ & Med & 25 \\
\hline Daucus carota L. & Zanahoria & Apiaceae & $E$ & $A-B$ & $\mathrm{D}$ & Com & 3 \\
\hline $\begin{array}{l}\text { Deyeuxia viridiflavescens (Poir.) } \\
\text { Kunth }\end{array}$ & Pasto penacho blanco & Poaceae & $\mathrm{N}$ & $\mathrm{P}$ & $\mathrm{D}$ & For & 3 \\
\hline $\begin{array}{l}\text { Dichondra microcalyx (Hallier f.). } \\
\text { Fabris }\end{array}$ & Oreja de ratón & Convolvulaceae & $\mathrm{N}$ & $\mathrm{P}$ & $\mathrm{D}$ & Ces-Med & 44 \\
\hline Digitaria sanguinalis (L.) Scop. & $\begin{array}{l}\text { Pata de gallina, pasto } \\
\text { cuaresma }\end{array}$ & Poaceae & $E$ & $A$ & M & For & 53 \\
\hline Dipsacus fullonum $\mathrm{L}$. & Cardo de cardar & Dipsacaceae & $E$ & $\mathrm{~B}$ & $\mathrm{D}$ & Med & 3 \\
\hline Distichlis spicata (L.) Greene & Pasto salado & Poaceae & $\mathrm{N}$ & $P$ & M & For & 3 \\
\hline Echinochloa colona (L.) Link & $\begin{array}{l}\text { Pasto colorado, } \\
\text { arrocillo }\end{array}$ & Poaceae & $\mathrm{C}$ & A & M & For & 56 \\
\hline $\begin{array}{l}\text { Echinochloa crus-galli (L.) P. } \\
\text { Beauv }\end{array}$ & $\begin{array}{l}\text { Capín arroz, pata de } \\
\text { gallo }\end{array}$ & Poaceae & $E$ & A & M & For & 84 \\
\hline $\begin{array}{l}\text { Echinochloa crus-pavonis (Kunth) } \\
\text { Schult. }\end{array}$ & $\begin{array}{l}\text { Chacrilla, arroz de } \\
\text { gallo }\end{array}$ & Poaceae & $E$ & A & $\mathrm{D}$ & Med & 19 \\
\hline Echium plantagineum L. & $\begin{array}{l}\text { Borraja chimarrona, } \\
\text { flor morada }\end{array}$ & Boraginaceae & $E$ & $A-B$ & $\mathrm{D}$ & For-Med & 12 \\
\hline $\begin{array}{l}\text { Eragrostis mexicana (Hornem) } \\
\text { Link ssp. Virescens }\end{array}$ & Paja voladora & Poaceae & $N$ & A & M & For & 53 \\
\hline
\end{tabular}


Tabla 3.1. (Continúa).

\begin{tabular}{|c|c|c|c|c|c|c|c|}
\hline Nombre científico & Nombre vulgar & Familia & Or & Cv & Mo & Val & Co \\
\hline Eruca vesicaria (L.) Cav. & Rúcula & Brassicaceae & $E$ & $A$ & $\mathrm{D}$ & Com-Med & 6 \\
\hline Eucaliptus sp. & Eucalipto & Myrtaceae & E & $P$ & $\mathrm{D}$ & Med & 9 \\
\hline Euphorbia maculata L. & $\begin{array}{l}\text { Hierba de la } \\
\text { golondrina }\end{array}$ & Euphorbiaceae & E & $P$ & $\mathrm{D}$ & Med & 3 \\
\hline Euphorbia serpens var microphylla & $\begin{array}{l}\text { Yerba meona, } \\
\text { lechetrés }\end{array}$ & Euphorbiaceae & $\mathrm{N}$ & $P$ & $\mathrm{D}$ & Com-Med & 22 \\
\hline Foeniculum vulgare Mill. & Hinojo & Apiaceae & $\mathrm{E}$ & $P$ & $\mathrm{D}$ & For-Med-Orn & 6 \\
\hline Fraxinus excelsior L. & Fresno & Oleaceae & E & $\mathrm{P}$ & $\mathrm{D}$ & Mad-Med & 3 \\
\hline Fumaria officinalis $\mathrm{L}$. & Flor de pajarito & Fumariaceae & E & A & $\mathrm{D}$ & Med & 3 \\
\hline Galinsoga parviflora Cav. & Albahaca silvestre & Asteraceae & $\mathrm{N}$ & A & $\mathrm{D}$ & For-Med & 94 \\
\hline $\begin{array}{l}\text { Gamochaeta platensis (Cabrera) } \\
\text { Cabrera }\end{array}$ & & Asteraceae & N & B-P & $\mathrm{D}$ & Med & 19 \\
\hline Geranium molle L. & Alfilerillo & Geraniaceae & $\mathrm{E}$ & A & $\mathrm{D}$ & Med & 9 \\
\hline Geranium rotundifolium L. & $\begin{array}{l}\text { Geranio de hoja } \\
\text { redonda }\end{array}$ & Geraniaceae & E & A & D & For & 3 \\
\hline Gleditsia triacanthos $\mathrm{L}$. & Acacia negra & Fabaceae & E & $P$ & D & & 3 \\
\hline Gomphrena celosioides Mart. & Peludilla & Amaranthaceae & $\mathrm{N}$ & $P$ & $\mathrm{D}$ & Med & 6 \\
\hline Grindelia pulchella Dunal & Melosilla- florcita & Asteraceae & $\mathrm{N}$ & $P$ & $\mathrm{D}$ & Med & 3 \\
\hline Hypochaeris radicata L. & $\begin{array}{l}\text { Roseta, achicoria de } \\
\text { monte }\end{array}$ & Asteraceae & E & $\mathrm{P}$ & D & Med-Com & 1 \\
\hline Jaborosa runcinata Lam. & Flor de sapo & Solanaceae & $\mathrm{N}$ & $P$ & $\mathrm{D}$ & & 19 \\
\hline Lactuca sativa $\mathrm{L}$. & Lechuga & Asteraceae & E & $A-B$ & $\mathrm{D}$ & Com-Med & 15 \\
\hline Lamium amplexicaule $\mathrm{L}$. & $\begin{array}{l}\text { Ortiga mansa, falsa } \\
\text { ortiga }\end{array}$ & Lamiaceae & E & $A-B$ & $\mathrm{D}$ & Com-Med-Me & 62 \\
\hline Lepidium didymum $\mathrm{L}$. & Mastuerzo & Brassicaceae & $\mathrm{N}$ & $A-B$ & $\mathrm{D}$ & Com-Med & 87 \\
\hline $\begin{array}{l}\text { Leptochloa chloridiformis (Hack.) } \\
\text { Parodi }\end{array}$ & Rhodes criollo & Poaceae & $\mathrm{N}$ & $P$ & M & & 56 \\
\hline Lolium multiflorum Lam. & Raigrás criollo & Poaceae & $\mathrm{E}$ & A & M & For-Ces & 50 \\
\hline Lonicera japonica Thunb & Madreselva & Caprifoliaceae & $\mathrm{E}$ & $P$ & $\mathrm{D}$ & Med-Orn & 3 \\
\hline Lotus glaber Mill. & Trébol pata de pájaro & Fabaceae & E & $P$ & $\mathrm{D}$ & For & 28 \\
\hline Lycopersicum esculentum Mill. & Tomate & Solanaceae & $\mathrm{N}$ & A & $\mathrm{D}$ & Com & 18 \\
\hline Lythrum hyssopifolia L. & Hierba del toro & Lythraceae & E & A & $\mathrm{D}$ & Med & 19 \\
\hline Malva neglecta Wallr. & Malva & Malvaceae & E & $P$ & $\mathrm{D}$ & Med-Com & 3 \\
\hline Malva parviflora L. & Malva & Malvaceae & E & $P$ & $\mathrm{D}$ & Med & 3 \\
\hline Matricaria chamomilla L. & Manzanilla & Asteraceae & E & A & $\mathrm{D}$ & Med & 69 \\
\hline Medicago lupulina L. & Trébol, lupulina & Fabaceae & E & $A-B$ & $\mathrm{D}$ & For-Med & 12 \\
\hline Medicago polymorpha L. & Trébol de carretilla & Fabaceae & E & A & $\mathrm{D}$ & For-Med & 6 \\
\hline Melilotus indicus (L.) All. & Trébol de olor & Fabaceae & E & A & $\mathrm{D}$ & For & 6 \\
\hline Melilotus officinalis (L.) Lam. & Trébol de olor amarillo & Fabaceae & E & $A-B$ & $\mathrm{D}$ & For-Med-Mel & 3 \\
\hline Mentha pulegium $L$. & Poleo & Lamiaceae & E & $P$ & $\mathrm{D}$ & Med & 3 \\
\hline Mentha suaveolens Ehrt. & Menta, mastranzo & Lamiaceae & E & $P$ & $\mathrm{D}$ & Com-Med & 3 \\
\hline Modiola caroliniana (L.) G. Don & Sanalotodo & Malvaceae & $\mathrm{N}$ & $\mathrm{P}$ & $\mathrm{D}$ & Med & 6 \\
\hline
\end{tabular}

Diversidad Vegetal Espontánea en Agroecosistemas Hortícolas de La Plata y su relación con diferentes estilos de Agricultura: Importancia para la sustentabilidad 
Tabla 3.1. (Continúa).

\begin{tabular}{|c|c|c|c|c|c|c|c|}
\hline Nombre científico & Nombre vulgar & Familia & Or & Cv & Mo & Val & Co \\
\hline Morus Alba L. & Mora blanca & Moraceace & $\mathrm{E}$ & $\mathrm{P}$ & D & Com-Med-Orn & 12 \\
\hline Nicotiana longiflora Cav. & Flor de sapo & Solanaceae & $\mathrm{N}$ & $\mathrm{P}$ & D & Med & 6 \\
\hline Origanum vulgare L. & Orégano & Lamiaceae & E & $P$ & $\mathrm{D}$ & Com-Med & 3 \\
\hline Oxalis articulata Savigny & Vinagrillo rosado, & Oxalidaceae & $\mathrm{N}$ & $P$ & $\mathrm{D}$ & Com-Med & 3 \\
\hline Oxalis corniculata L. & Vinagrillo & Oxalidaceae & C & $P$ & $\mathrm{D}$ & Com-Med & 15 \\
\hline Oxalis sp.1 & & Oxalidaceae & & & $\mathrm{D}$ & & 3 \\
\hline Oxalis sp.2 & & Oxalidaceae & & & D & & 3 \\
\hline $\begin{array}{l}\text { Oxypetalum solanoides Hook. \& } \\
\text { Arn. }\end{array}$ & Plumerillo & Apocynaceae & N & $P$ & $\mathrm{D}$ & & 3 \\
\hline Panicum bergii Arechav. & Paja voladora & Poaceae & $\mathrm{N}$ & $\mathrm{P}$ & M & & 16 \\
\hline Paspalum dilatatum Poir. & Pasto miel & Poaceae & $\mathrm{N}$ & $P$ & M & For & 12 \\
\hline Parthenium hysterophorus L. & Escoba amarga & Asteraceae & $\mathrm{N}$ & A & D & Med & 2 \\
\hline $\begin{array}{l}\text { Petroselinum crispum (Mill.) A.W. } \\
\text { Hill }\end{array}$ & Perejil & Apiaceae & E & B & D & Com-Med & 3 \\
\hline Phaseolus vulgaris $\mathrm{L}$. & Poroto & Fabaceae & N & $P$ & $\mathrm{D}$ & Com & 3 \\
\hline Phyla canescens (Kunth) Greene & Hierba de mosquito & Verbenaceae & N & $P$ & D & For & 6 \\
\hline Physalis viscosa L. & Camanbú & Solanaceae & $\mathrm{N}$ & $P$ & $\mathrm{D}$ & Med-For & 6 \\
\hline Picris echioides L. & Pega-pega & Asteraceae & $\mathrm{N}$ & $P$ & $\mathrm{D}$ & Med-For & 6 \\
\hline Plantago lanceolata L. & Plantago, falso llantén & Plantaginaceae & $\mathrm{E}$ & $P$ & $\mathrm{D}$ & Com-Med-Orn & 12 \\
\hline Pluchea sagittalis (Lam.) Cabrera & $\begin{array}{l}\text { Lucera, yerba del } \\
\text { lucero }\end{array}$ & Asteraceae & $\mathrm{N}$ & $\mathrm{P}$ & D & Med & 3 \\
\hline Poa annua L. & Pastito de invierno & Poaceae & $\mathrm{E}$ & A & D & For & 50 \\
\hline Polygonum aviculare $\mathrm{L}$. & $\begin{array}{l}\text { Sanguinaria, cien } \\
\text { nudo }\end{array}$ & Polygonaceae & E & $A-B$ & D & Med & 53 \\
\hline Polygonum persicaria L. & Lagunilla & Polygonaceae & E & A & $\mathrm{D}$ & Com & 9 \\
\hline Portulaca oleracea L. & Verdolaga & Portulacaceae & C & A & $\mathrm{D}$ & Com-Med & 90 \\
\hline Potentilla vesca (L.) Scop. & Frutilla & Rosaceae & E & $P$ & D & Com & 6 \\
\hline Raphanus sativus L. & Nabón, rabanito & Brassicaceae & E & $A-B$ & $\mathrm{D}$ & Med & 6 \\
\hline Rapistrum rugosum (L.) All. & Mostacilla & Brassicaceae & E & A & $\mathrm{D}$ & Com & 3 \\
\hline $\begin{array}{l}\text { Rorippa bonariensis (Poir.) } \\
\text { Macloskie }\end{array}$ & $\begin{array}{l}\text { Mastuerzo de los } \\
\text { sapos }\end{array}$ & Brassicaceae & $\mathrm{N}$ & $A-B$ & $\mathrm{D}$ & Mad-Meli-Orn & 3 \\
\hline Rumex crispus L. & Lengua de vaca & Polygonaceae & E & $P$ & $\mathrm{D}$ & Com-For-Med & 69 \\
\hline Salpichroa origanifolia (Lam.) Baill. & Huevito de gallo & Solanaceae & $\mathrm{N}$ & $P$ & $\mathrm{D}$ & Com-Med-Mel & 12 \\
\hline Senecio vulgaris $\mathrm{L}$. & Senecio común & Asteraceae & $\mathrm{E}$ & A & $\mathrm{D}$ & Med & 56 \\
\hline Setaria parviflora (Poir.) Kerguélen & $\begin{array}{l}\text { Cola de zorro, } \\
\text { plumerillo }\end{array}$ & Poaceae & E & A & $\mathrm{D}$ & For & 9 \\
\hline Setaria verticillata (L.) P. Beauv. & Pega-pega & Poaceae & $\mathrm{E}$ & A & D & For & 12 \\
\hline Sida cordifolia L. & $\begin{array}{l}\text { Escobilla, escoba } \\
\text { negra }\end{array}$ & Malvaceae & $\mathrm{N}$ & $P$ & $\mathrm{D}$ & Med & 12 \\
\hline Sida rhombifolia L. & Afata & Malvaceae & $\mathrm{N}$ & A-P & D & Med & 9 \\
\hline Silene gallica $\mathrm{L}$. & Calabacilla & Caryophyllaceae & E & A & D & & 3 \\
\hline
\end{tabular}

Diversidad Vegetal Espontánea en Agroecosistemas Hortícolas de La Plata y su relación con diferentes estilos de Agricultura: Importancia para la sustentabilidad 
Tabla 3.1. (Continúa).

\begin{tabular}{|c|c|c|c|c|c|c|c|}
\hline Nombre científico & Nombre vulgar & Familia & Or & Cv & Mo & Val & Co \\
\hline Solanum chacoense Bitter & $\begin{array}{l}\text { Pegajosa, solanum } \\
\text { pegajoso }\end{array}$ & Solanaceae & $\mathrm{N}$ & $P$ & $\mathrm{D}$ & Com & 3 \\
\hline Solanum chenopodioides Lam. & $\begin{array}{l}\text { Yerba mora, yerba } \\
\text { buena }\end{array}$ & Solanaceae & $\mathrm{N}$ & A & D & For & 12 \\
\hline Solanum melongena $\mathrm{L}$ & Berenjena & Solanaceae & $\mathrm{E}$ & A-P & D & Com-Med & 3 \\
\hline $\begin{array}{l}\text { Solanum pygmaeum Cav. var } \\
\text { pygmaeum. }\end{array}$ & & Solanaceae & $\mathrm{N}$ & $\mathrm{P}$ & $\mathrm{D}$ & & 6 \\
\hline Solanum sarrachoides Sendtn & Pegajosa & Solanaceae & $\mathrm{N}$ & A & D & & 3 \\
\hline Solanum sisymbriifolium Lam. & $\begin{array}{l}\text { Espina colorada, tutía, } \\
\text { revienta caballo }\end{array}$ & Solanaceae & $\mathrm{N}$ & $\mathrm{P}$ & $\mathrm{D}$ & For & 6 \\
\hline Sonchus oleraceus L. & Cerraja & Asteraceae & $\mathrm{E}$ & A & D & Com-For-Med & 97 \\
\hline Spergula villosa Pers. & & Caryophyllaceae & $\mathrm{N}$ & $\mathrm{P}$ & D & Med & 3 \\
\hline Stachys arvensis (L.) L. & $\begin{array}{l}\text { Ortiga mansa, Hierba } \\
\text { de gato }\end{array}$ & Lamiaceae & $\mathrm{E}$ & A & D & Med & 25 \\
\hline $\begin{array}{l}\text { Stellaria media var. media (L.) } \\
\text { Cirillo }\end{array}$ & Capiquí & Caryophyllaceae & E & A & D & Med- Com & 66 \\
\hline Tagetes erecta $\mathrm{L}$. & Copete & Asteraceae & $\mathrm{E}$ & A & D & Med-Orn & 3 \\
\hline $\begin{array}{l}\text { Taraxacum officinale G. Weber ex } \\
\text { F.H. Wigg. }\end{array}$ & Diente de león & Asteraceae & $\mathrm{E}$ & $\mathrm{P}$ & D & Med-Com & 37 \\
\hline Thlaspi arvense L. & Carraspique & Brassicaceae & $\mathrm{E}$ & A & D & Med & 3 \\
\hline Trifolium pratense L. & Trébol rojo & Fabaceae & $\mathrm{E}$ & $P$ & D & For-Mel & 12 \\
\hline Trifolium repens $\mathrm{L}$. & Trébol blanco & Fabaceae & $\mathrm{E}$ & $\mathrm{P}$ & D & For-Mel-Ces & 60 \\
\hline $\begin{array}{l}\text { Urochloa platyphylla (Munro ex C. } \\
\text { Wright) R.D. Webster }\end{array}$ & Pasto bandera & Poaceae & $\mathrm{N}$ & A & M & & 3 \\
\hline Urtica urens $\mathrm{L}$. & Ortiga & Urticaceae & $\mathrm{E}$ & A & D & Com-Med & 84 \\
\hline $\begin{array}{l}\text { Verbena bonariensis L. var } \\
\text { bonariensis }\end{array}$ & Verbena & Verbenaceae & $\mathrm{N}$ & $A-B$ & $\mathrm{D}$ & Mel-Orn & 3 \\
\hline Verbena litoralis Kunth var litoralis & Verbena de litoral & Verbenaceae & $\mathrm{N}$ & $\mathrm{P}$ & D & Med & 3 \\
\hline $\begin{array}{l}\text { Verbesina encelioides (Cav.) } \\
\text { Benth. \& Hook. f. ex A. Gray }\end{array}$ & Girasolcito & Verbenaceae & $\mathrm{N}$ & A & $\mathrm{D}$ & Med & 3 \\
\hline $\begin{array}{l}\text { Veronica peregrina L. ssp. } \\
\text { Xalapensis (Kunth) Pennell }\end{array}$ & $\begin{array}{l}\text { Verónica, } \\
\text { Canchalagua }\end{array}$ & Plantaginaceae & $\mathrm{E}$ & A & D & Com-Med & 72 \\
\hline Veronica persica L. & $\begin{array}{l}\text { Verónica, } \\
\text { Canchalagua }\end{array}$ & Plantaginaceae & $\mathrm{E}$ & A & D & Orn & 16 \\
\hline Vicia sativa L. & Vicia & Fabaceae & $\mathrm{E}$ & A & D & For & 16 \\
\hline $\begin{array}{l}\text { Wedelia glauca (Ortega) O. Hoffm. } \\
\text { ex Hicken }\end{array}$ & Sunchillo & Asteraceae & N & $\mathrm{P}$ & $\mathrm{D}$ & & 12 \\
\hline $\begin{array}{l}\text { Xanthium ambrosioides Hook. \& } \\
\text { Arn. }\end{array}$ & Abrojo chico, abrojillo & Asteraceae & $\mathrm{N}$ & A & $\mathrm{D}$ & Med & 1 \\
\hline Xanthium cavanillesii Schouw & Abrojo, abrojo grande & Asteraceae & $\mathrm{N}$ & A & D & Com-Med & 1 \\
\hline
\end{tabular}

$\left({ }^{*}\right)$ Pteridophyta

\subsubsection{Importancia de las especies y estructura de grupos funcionales}

Las especies de mayor constancia (> 70\%) fueron, en orden de importancia decreciente, Sonchus oleraceus, Galinsoga parviflora, Portulaca oleracea, Lepidium didymum, Urtica urens, Echinochloa crus-galli, Cynodon dactylon, Verónica peregrina 
(Tabla 3.1). Entre 60 y $70 \%$ se encontraron Rumex crispus, Chenopodium album, Matricaria recutita, Convolvulus arvensis, Rumex crispus, Stellaria media var. Media, Lamium amplexicaule, Capsella bursa-pastori . La mayoría de estas especies fueron anuales y dicotiledóneas.

Algunas especies resultaron de importancia en el control de plagas en la zona. Por ejemplo, Matricaria chamomilla, Ammi majus y Trifolium repens, se asocian con la presencia de una alta diversidad enemigos naturales de trips (Saini \& Polack, 2002).

La proporción de exóticas superó a las especies nativas en un 10,4\% (Tabla 3.2), sin embargo, estas últimas estuvieron bien representadas (44\%). La cantidad de especies anuales y perennes fue similar. Las especies bienales y perennes sumadas representaron un $55 \%$ de abundancia relativa. La abundancia relativa de dicotiledóneas superó ampliamente a las monocotiledóneas.

Tabla 3.2. Origen, ciclo de vida y morfología de plantas espontáneas en el Cinturón Hortícola de La Plata.

\begin{tabular}{|c|c|c|}
\hline & Categoría & Abundancia relativa (\%) \\
\hline \multirow[t]{3}{*}{ Origen } & Nativas & 44,4 \\
\hline & Exóticas & 54,8 \\
\hline & Cosmopolitas & 0,7 \\
\hline \multirow[t]{3}{*}{ Ciclo de vida } & Anuales & 41,9 \\
\hline & Bienales & 13,5 \\
\hline & Perennes & 44,6 \\
\hline \multirow[t]{2}{*}{ Morfología } & Dicotiledóneas & 90,5 \\
\hline & Monocotiledóneas & 9,3 \\
\hline
\end{tabular}

\subsubsection{Valor utilitario}

Las especies encontradas presentaron un significativo valor utilitario. El $89 \%$ de las especies presentó al menos un valor utilitario. Los valores de uso principales fueron el medicinal, comestible, forrajero, ornamental, melifero y maderero. El valor de uso medicinal $(72,9 \%)$ y el comestible $(36,4 \%)$, fueron los más destacados (Figura 3.5$)$.

Diversidad Vegetal Espontánea en Agroecosistemas Hortícolas de La Plata y su relación con diferentes estilos de Agricultura: Importancia 


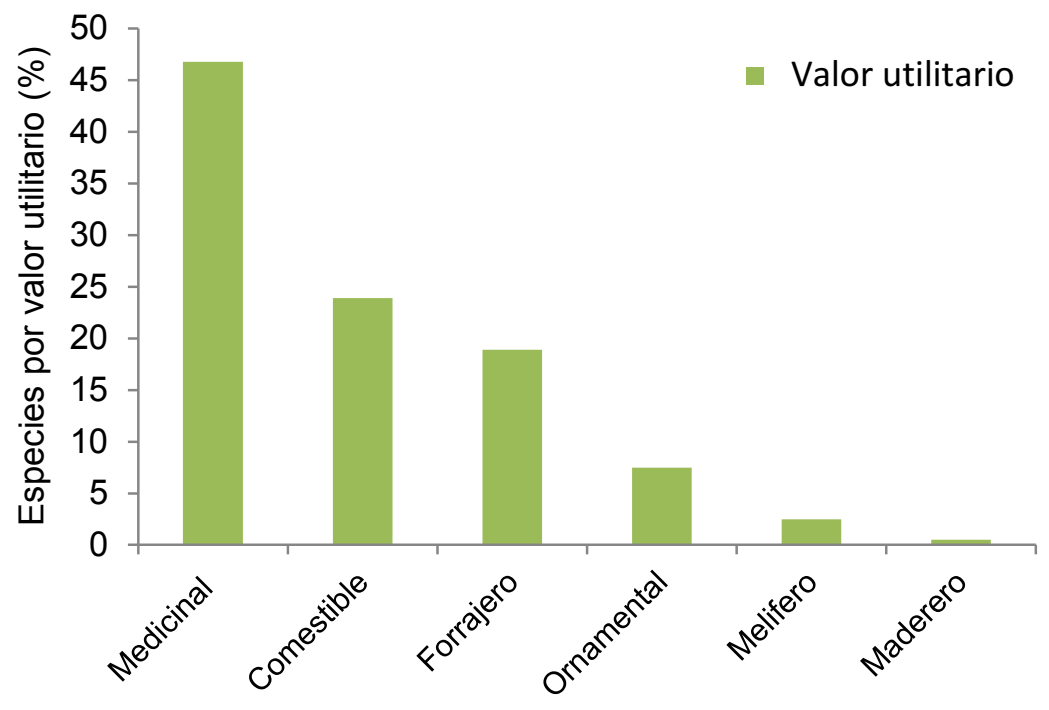

Figura 3.5. Proporción de especies espontáneas por valor utilitario.

\subsubsection{Percepción de los agricultores acerca de las espontáneas}

Se observó una diversidad de opiniones de los agricultores sobre la vegetación espontánea. Los aspectos más importantes percibidos como positivos fueron, el aporte de materia orgánica al suelo (35\%) y el refugio de insectos benéficos (21\%) (Figura 3.6). Algunos agricultores, además, identificaron los siguientes aspectos acerca de la vegetación espontánea que no fueron considerados previamente en la entrevista: protegen el cultivo contra el sol, mejoran la estructura al suelo, proveen alimento alternativo para las plagas. En general, se observó una significativa falta de conocimiento de los atributos de la vegetación espontánea, como se observa en las barras verdes de mayor altura o falta de aprovechamiento de esos beneficios (barras rojas). 


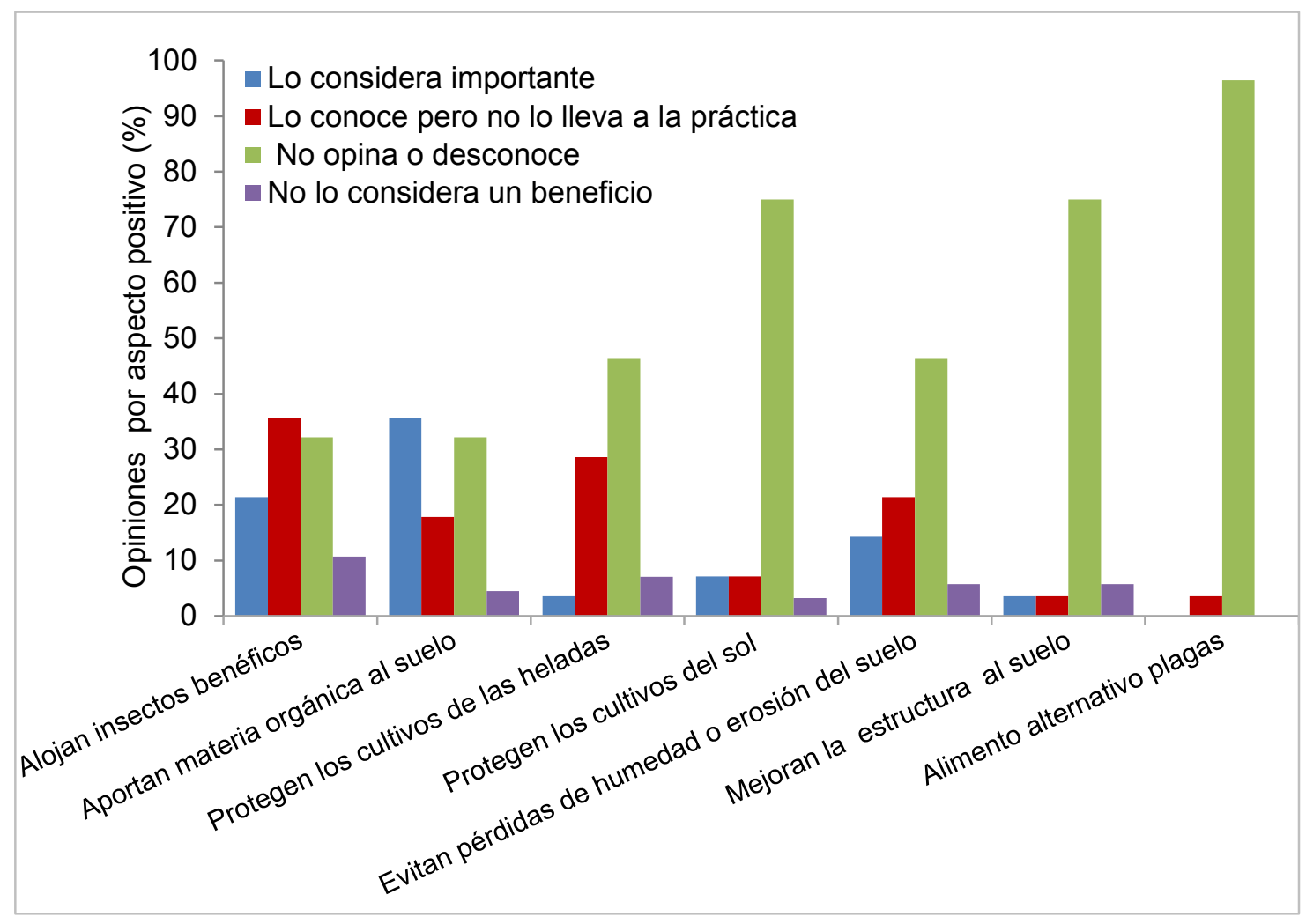

Figura 3.6. Dentro de cada aspecto positivo de la vegetación espontánea, las barras indican el porcentaje de agricultores que: consideran importante el aspecto señalado; lo conocen; no opinan o desconocen; no lo consideran un beneficio, respectivamente.

Los principales aspectos negativos destacados por los agricultores acerca de la presencia de vegetación espontánea fueron, que le quitan los nutrientes a los cultivos (54\%), que albergan plagas de los cultivos (43\%) y que molestan en las prácticas de labranza (29\%) (Figura 3.7). Los agricultores además indicaron que implican un costo económico, le quitan el agua al cultivo, le quitan "espacio" y "ventilación" al cultivo, son de crecimiento rápido. Estos aspectos no fueron considerados previamente en la entrevista. 


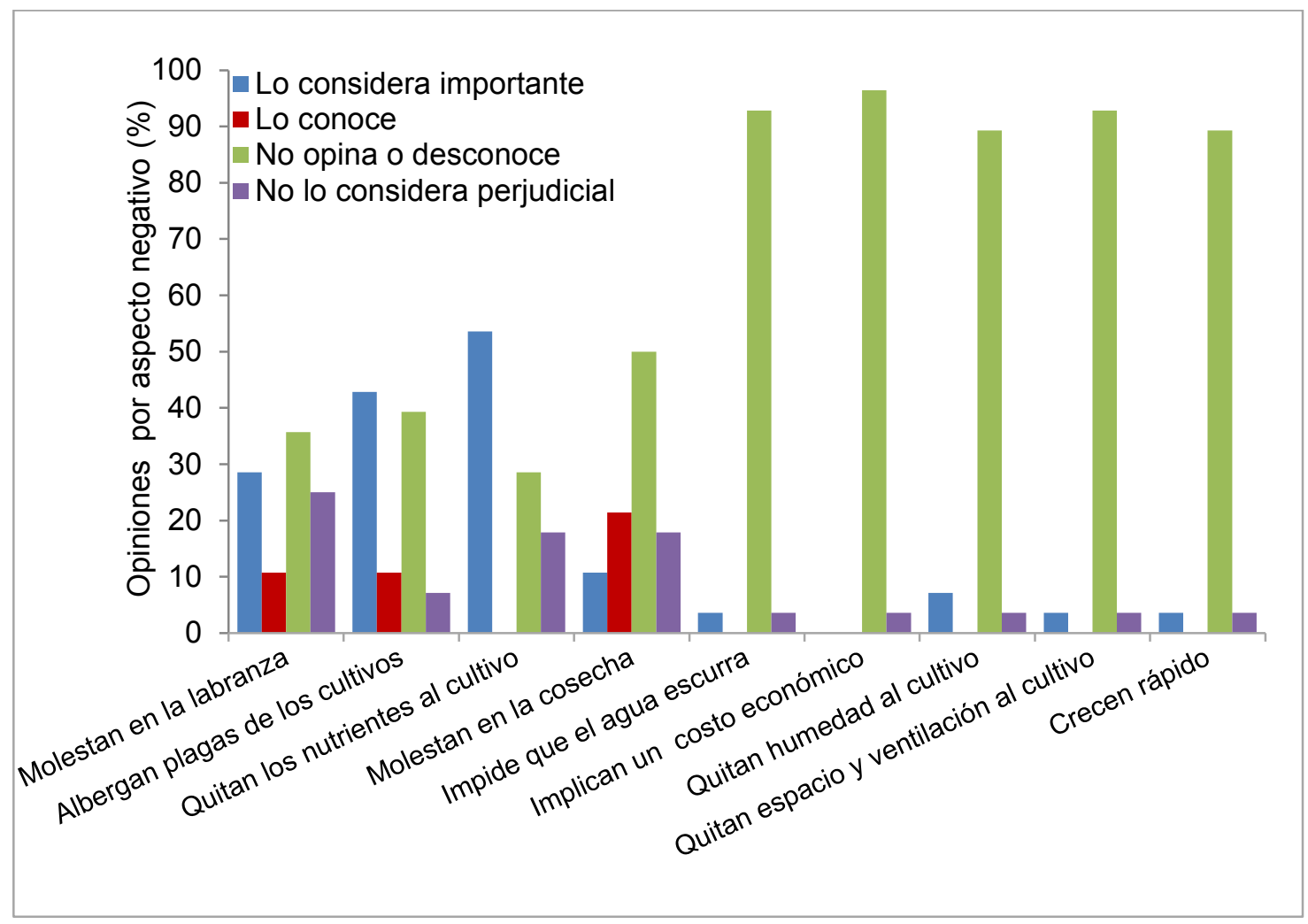

Figura 3.7. Dentro de cada aspecto negativo de la vegetación espontánea, las barras indican el porcentaje de agricultores que: consideran importante el aspecto señalado; lo conocen; no opinan o desconocen o no lo consideran un aspecto perjudicial, respectivamente.

Para un mismo aspecto, planteado como beneficio o no, la importancia que se le otorgó al perjuicio de la vegetación espontánea fue superior que el beneficio. Tal es el caso del aporte de materia orgánica al suelo y de la quita de nutrientes al cultivo (Figura 3.6 y 3.7). Lo mismo ocurrió entre el albergue de plagas en la vegetación y el beneficio de ser hábitat de enemigos naturales.

Durante la entrevista surgieron diferentes opiniones acerca de los aspectos analizados (Tabla 3.3). Los resultados muestran una mayor tendencia a considerar a la vegetación espontánea como perjudicial. Esta percepción se centró en cuestiones económicas ante la posibilidad de afectar el rendimiento del cultivo, tal como el posible ataque de plagas y la competencia por distintos recursos (Figura 3.7). Además, se observaron cuestiones estéticas asociadas a la necesidad de mantener limpia la tierra de plantas no cultivadas (Tabla 3.3). 
Tabla 3.3. Frases más destacadas mencionadas textualmente por los agricultores agrupados según las consideraciones positivas y negativas que tuvieron sobre la vegetación espontánea.

\section{Consideraciones negativas}

-"Nosotros desde ya las detestamos, pero algo tienen que tener. La tierra que no da maleza no sirve tampoco para verdura".

-"No me gusta por una cuestión de higiene visual".

-"Es mejor tener limpia la tierra, queda más prolijo".

-"Nosotros sembramos los cultivos, a la maleza la siembra el diablo".

-"La maleza es un gasto nomás".

-"Vos las plantaciones, igual que una casa, más limpias las tenés hay más posibilidades de todo".

-"Alojan a los malos también. Prefiero que no estén los benéficos pero tampoco estén las malezas".

-"Cuando se crece la verdolaga (Portulaca oleracea) no muere, aunque la cortamos vuelve a aparecer, me molesta en que continua su crecimiento".

-"Son contraproducentes por donde la mires. El nutriente en vez que lo consuma la verdura lo tiene que compartir con la maleza".

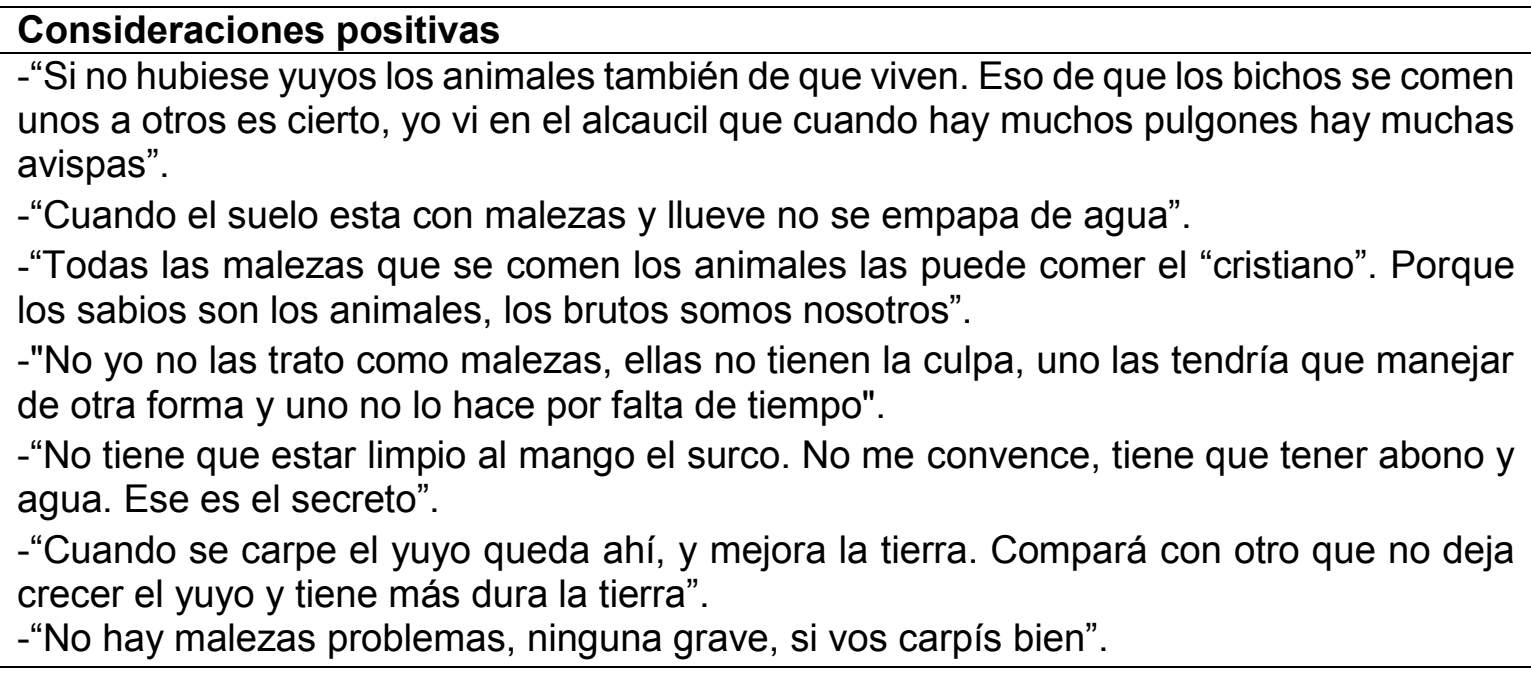

El 57\% de los agricultores respondió que les "molesta" la vegetación espontánea y el $32 \%$ que les "molesta" relativamente. Estos últimos indicaron que solo les molestan algunas especies o que no les molestan, mientras estén controladas. El 11\% afirmó estar más preocupados por las plagas. El nivel de "molestia" se reflejó en diversas opiniones como:

"Lo que me preocupa a mí son los dos bichos más bravos que tenemos que son la flor blanca, la enredadera y el cebollín";

Diversidad Vegetal Espontánea en Agroecosistemas Hortícolas de La Plata y su relación con diferentes estilos de Agricultura: Importancia 
"No es que me preocupan llevan tiempo, son una realidad, lo que pasa es que la tierra siempre la moves";

"A veces me preocupan cuando ya la van ganando al cultivo, nada más";

"Por ahora un problema porque la tierra siempre está llena de semillas que crecen";

"Es un drama, siempre aparecen y cuando llueve más";

"No las dejo criar por eso no llegan a molestar",

"No, con el bromuro está limpito, lo único que nace es trébol";

"Todas me molestan".

"Los invernáculos tiene que estar cero de malezas. El insecto tiene que estar lo más lejos posible, sino da el zarpazo".

"No tiene que estar limpio al mango el surco, tiene que tener abono y agua. Ese es el secreto".

El $75 \%$ de los agricultores no reconoció algún valor de uso de las plantas. Algunos manifestaron usar manzanilla y menta para el té, malva, cerraja para alimentar animales, preparados de ortiga como insecticida y para rellenar el suelo en el invernáculo. En un caso, había gente que visitaba la quinta para llevar verdolaga como alimento, y carqueja para el hígado. En relación a esto el agricultor expresó "eso no sé si llamarlo maleza". Además algunos manifestaron utilizar, en tiempos anteriores, yuyo colorado y verdolaga como alimento y, plantas para tapar el zapallo contra las heladas. En un caso se utilizaba quínoa para las lastimaduras.

\subsection{DISCUSIÓN}

\subsubsection{Caracterización de la biodiversidad presente en las fincas}

Los resultados confirman que los sistemas hortícolas de La Plata albergan una importante riqueza florística, que se sustenta en el significativo número encontrado de especies, géneros y familias de plantas espontáneas y con una importante proporción de especies con valor utilitario. La riqueza de especies en los sistemas hortícolas analizados (152 especies en 178 ha) resulta elevada si se compara con cultivos de grano de amplias superficies dominadas por pocos cultivos. En la Pampa Ondulada, de La Fuente et al. (1999) reportaron 60 especies en un área total de 6000 ha, y Poggio et al. (2004) encontraron 96 especies en una superficie de 2000 ha. En el sur de la provincia de Buenos

Diversidad Vegetal Espontánea en Agroecosistemas Hortícolas de La Plata y su relación con diferentes estilos de Agricultura: Importancia 
Aires, Scursoni et al. (2014), encontraron 114 especies de espontáneas en un área relevada de 22100 ha (un total de 372 campos con un promedio de 70 ha relevadas en cada uno).

Las especies exóticas, anuales y dicotiledóneas son características de sistemas de alta intensidad y frecuente disturbio, pioneras en sucesiones secundarias (Zanin et al., 1997, Suárez et al. 2001; Urban, 2010). En los sistemas de cultivos las invasiones de exóticas se vuelven comunes al alterar la comunidad de plantas nativas de los pastizales originales (Ghersa \& León 1999; Martínez- Ghersa et al., 2000; Guglielmini et al., 2007). Las especies exóticas relevadas superaron a las nativas, aunque en baja proporción (10,4 \%). En cambio, en otros trabajos fueron superiores las diferencias en el porcentaje de exóticas, tanto en cultivos hortícolas, como en sistemas extensivos de monocultivo (entre un 70 a $80 \%$ de exóticas) (Poggio, 2004; Cerazo \& Conticello, 2008). En cuanto a la morfología, las dicotiledóneas superaron ampliamente a las monocotiledóneas (90\%), coincidiendo con lo encontrado en otros trabajos (Poggio, 2004; de la Fuente et al, 2006; Cerazo \& Conticello, 2008). La presencia de dicotiledóneas se atribuye a que se adaptan mejor a niveles bajos de luz en cultivos con alta productividad respecto de las monocotiledóneas (Suarez et al., 2001).

Las especies anuales se asocian con sistemas donde el suelo es arado y/o con un sistema de manejo intensivo, con uso de herbicidas de amplio espectro (Ghersa \& Martinez Ghersa, 2000; Suarez et. al., 2001). Por lo contrario, la presencia de especies de ciclo de vida largo son características de ambientes semi disturbados o de disturbios intermedios (Grime, 1979). Por ejemplo, en agroecosistemas pampeanos sin remoción del suelo, predominaron las especies perennes (Suárez et al., 2006). En el área de estudio, la proporción de especies anuales no presentó diferencias con las perennes, siendo importante el número de especies de ciclo de vida largo, como sugiere el total de bienalesperennes. En cambio, en el trabajo de Cerazo \& Conticello (2008) en cultivos hortícolas, las presencia de anuales fue muy superior. Poggio (2005) encontró un mayor número de anuales tanto en monocultivos, como intercultivos combinando las mismas especies, sin embargo, las perennes se destacaron en sistemas de intercultivos. Las menores diferencias encontradas entre la proporción de exóticas y nativas y, anuales y perennes, sugieren que las fincas estudiadas difieren en los niveles de disturbio determinando una flora compuesta de especies propias de etapas pioneras y especies de etapas algo más avanzadas en la sucesión ecológica.

Diversidad Vegetal Espontánea en Agroecosistemas Hortícolas de La Plata y su relación con diferentes estilos de Agricultura: Importancia 
Los aspectos discutidos anteriormente sugieren que la tendencia a una homogenización del ambiente por las prácticas culturales y una baja diversidad cultivada puede comprometer la diversidad de especies espontáneas y sus funciones asociadas que son importantes para sostener organismos de otros niveles tróficos (de la Fuente et al., 2006). Por el contrario, el incremento de la diversidad de espontáneas se asocia con el incremento de la heterogeneidad de hábitats y la disminución de la intensidad de la agricultura (Benton et al., 2003; Hole et al., 2005, Stupino et al., 2008; Stupino et al., 2014). En el área estudiada, existirían diferentes niveles de disturbio, representados por fincas bajo diferente manejo que influye de manera particular sobre la diversidad y composición florística a nivel zonal. Los estilos de agricultura que implican un disturbio intermedio y que mantienen una significativa diversidad cultivada, podrían contribuir a una mayor conservación de la diversidad de plantas espontáneas, mantener una flora de especies nativas y de ciclo de vida largo.

\subsubsection{Percepción de los agricultores acerca de la vegetación espontánea}

Además de lo señalado anteriormente, la presencia de plantas espontáneas en las fincas podría depender, en parte, de la percepción que los agricultores tienen acerca de las mismas. Los resultados encontrados muestran que en los agricultores de la zona de La Plata existe una dominante valoración de las especies espontáneas como algo perjudicial para los cultivos, validando la segunda hipótesis. En esta valoración negativa predominó el criterio económico de eliminarlas por afectar el rendimiento de los cultivos. Además, se identificó la preocupación por la interferencia en las labores y el criterio estético de mantener el campo "limpio" porque queda más prolijo. El campo libre de espontáneas tiene un sentido de habilidad y dedicación del agricultor que debe ser visto por la comunidad (Soini \& Akkula, 2007) y que, por el contrario, la falta de tiempo en esa tarea representa una incomodidad manifiesta, algo así como "tener la casa ordenada cuando llega una persona de visita".

En cuanto a las opiniones dadas por los agricultores acerca de si les molestan las malezas, se detectaron dos categorías cualitativas, que dichas plantas les "molestan" en niveles significativos o en niveles menores, "molestia intermedia". Esto, sugiere que existen diferencias entre los agricultores en la tolerancia a las malezas, coincidiendo con otro trabajo (Ahnström, 2009). En niveles bajos de tolerancia ("bajo umbral de malezas") la decisión de control de las espontáneas puede ser más relevante.

Diversidad Vegetal Espontánea en Agroecosistemas Hortícolas de La Plata y su relación con diferentes estilos de Agricultura: Importancia 
Los aspectos indicados, confirman el predominio del concepto tradicional de maleza como, "plantas que llegan a ser perjudiciales o indeseables en determinado tiempo y lugar" (Marzocca, 1976). Si bien, se reconocieron aspectos positivos, los agricultores presentaron escaso conocimiento o falta de puesta en práctica de los mismos (barras verdes y rojas, respectivamente, de la figura 3.6). Los aspectos identificados se centraron en el atributo de las plantas de incorporar materia orgánica y alojar insectos benéficos.

Se encontró una alta proporción de especies espontáneas con valor utilitario, aunque pocos agricultores manifestaron su utilización con distintos fines como, medicinal, alimento propio o de los animales y de utilidad en las prácticas de manejo. Algunos de esos usos fueron realizados en años anteriores y ya no se tenían en cuenta.

Los resultados muestran un contraste entre el discurso (lo que se dice) y la práctica (lo que se hace), debido a la ausencia de estrategias de manejo implementadas para potenciar aquellos aspectos positivos de la vegetación espontánea que fueron reconocidos por los agricultores. Esto sugiere que los servicios ecológicos podrían ser visualizados como beneficios indirectos y de difícil aplicación en el corto plazo, priorizándose el criterio de reducir las variables que afectan directamente el rendimiento de los cultivos. Esto coincide con Kelemen et al. (2013) quienes observaron que los agricultores reconocen ciertos beneficios de la biodiversidad que asocia directamente a la actividad productiva (por ejemplo, como alimento para los animales), pero al mismo tiempo identifican que generan costos y causan pérdida de los cultivos. Cuando la percepción negativa adquiere un mayor peso influye en la decisión de control (Mace et al., 2007; Wilson et al., 2008).

La valoración de las especies espontáneas como algo perjudicial y sin valor de uso constituye un importante riesgo para la biodiversidad, aunque no siempre se traduce en su eliminación, debido al costo que representa (Ahnström, 2009). Si los costos disminuyeran la biodiversidad podría verse afectada por las prácticas que tienden a disminuir su presencia y/o abundancia.

En este capítulo se analizó el conjunto de fincas relevadas. Sin embargo, la contribución de un agroecosistema a la conservación de la biodiversidad podría variar según el estilo de agricultura que se realice y la lógica del agricultor. En estudios previos se encontraron diferencias en la biodiversidad entre fincas orgánicas y convencionales siendo

Diversidad Vegetal Espontánea en Agroecosistemas Hortícolas de La Plata y su relación con diferentes estilos de Agricultura: Importancia 
mayor en las orgánicas (van Elsen, 2000; Hyvonen et al., 2003; Bengtsson et al., 2005; Hole et al., 2005; Stupino et al., 2008; Gabriel et al., 2010; Rahmann, 2011; Henckel et al., 2015). Los agricultores que producen de forma orgánica podrían diferir en la conceptualización de la misma respecto de los convencionales (Soini \& Akkula, 2007; Kelemen et al., 2013; Kross et al., 2017). Por lo tanto, se requiere un análisis comparativo entre los distintos estilos de agricultura de la zona, que permita establecer posibles diferencias en la diversidad, composición florística y grupos funcionales asociados. Estos aspectos comparados serán analizados en los siguientes capítulos.

\subsection{CONCLUSIONES}

Los resultados de este capítulo muestran, que el Cinturón Hortícola de La Plata alberga una importante riqueza florística. Esto se corresponde con el alto número encontrado de especies, géneros y familias de plantas espontáneas y con una importante proporción de especies con valor utilitario. Esta riqueza resultó superior a otras formas de producción hortícola y extensiva, probablemente por la heterogeneidad que presenta el Cinturón Hortícola Platense en cuanto a la diversidad cultivada, y diferentes formas de manejo.

Los resultados presentados confirman que en el área de estudio, prevalece el concepto tradicional de maleza, que se ve reflejado en una dominante valoración de las especies espontáneas como algo perjudicial o negativo por parte de los agricultores. No obstante, esta percepción presentó diferencias entre los agricultores en cuanto al grado de preocupación o molestia generada por dicha vegetación. El reconocimiento de los beneficios, servicios ecológicos y valores de uso de la vegetación espontánea no se trasladó en prácticas concretas para potenciarlos o hubo poco conocimiento de los mismos. El predominio de la percepción negativa de la vegetación espontánea podría poner en riesgo la biodiversidad especialmente cuando los insumos se vuelven menos costosos y de más fácil acceso.

La contribución a la conservación de la biodiversidad vegetal de cada finca puede variar según el estilo de agricultura que se realice y la lógica del agricultor. Las fincas estudiadas podrían presentar diferencias en el nivel de disturbio, determinando la presencia de especies con distintos grupos funcionales, en proporciones indicativas de estadios

Diversidad Vegetal Espontánea en Agroecosistemas Hortícolas de La Plata y su relación con diferentes estilos de Agricultura: Importancia 
sucesionales diferentes. Aquellos estilos de agricultura que impliquen un disturbio menor pueden contribuir en mayor medida a mantener la biodiversidad general. Esto se podría corresponder con un agricultor menos preocupado por las especies espontáneas.

Las posibles diferencias en la diversidad entre sistemas bajo diferente manejo se analizan en los siguientes capítulos. En el Capítulo 4 se caracterizan los estilos de agricultura presentes en el Cinturón Hortícola según el manejo de los cultivos. En los siguientes capítulos se analiza cuál es la diversidad de espontáneas presente a diferentes escalas espaciales y, cómo influyen las distintas formas de manejo encontradas sobre la misma.

\subsection{BIBLIOGRAFÍA}

Ahnström, J. 2009. Farmland biodiversity - in the hands and minds of farmers. Effects of landscape structure, management and the farmer's interest in nature. Thesis Swedish University of Agricultural Sciences, Uppsala.116 p.

Albrecht H. 2003. Suitability of arable weeds as indicator organisms to evaluate species conservation effects of management in agricultural ecosystems. Agriculture, Ecosystems and Environment 98: 201-211.

Altieri MA. 1999. The ecological role of biodiversity in agroecosystems. Agriculture, Ecosystems and Environment 74: 19-31.

Altieri MA \& Nicholls CA. 1999. Biodiversity, Ecosystem Function, and Insect Management in Agricultural Systems. En: Biodiversity in Agroecosystems WW Collins \& CO Qualset (Eds.), CRC Press LLC. 5: 69-84.

Ander-Egg E. 1985. Metodología del trabajo social. Editorial El Ateneo, México. 244 p.

Archenti A, Ringuelet R \& Salva MC. 1993. Los procesos de diferenciación de los productores hortícolas de La Plata. Etnia $N^{\circ} 38 / 39$.

Banks J. 2004. Divided Culture: Integrating and Conservation Biology Agriculture Environmental Science, Interdisciplinary Arts and Sciences 2(10): 537-545. Disponible en: http://digitalcommons.tacoma.uw.edu/ias_pub/3.

Barsky A. 2005. El periurbano productivo, un espacio en constante transformación. Introducción al estado del debate, con referencias al caso de Buenos Aires. Scripta Nova cripta Nova, Revista Electrónica de Geografía y Ciencias Sociales 9(194): 36. Disponible en: http://www.ub.es/geocrit/sn/sn-194-36.htm. 
Bátary P, Báldi A, Kleijn D \& Tscharntke T. 2011. Landscape-moderated biodiversity effects of agri-environmental management: a meta-analysis. Proceedings of de Royal Society B 278: 1894-1902.

Bengtsson J, Ahnstrom J \& Weibull AC. 2005. The effects of organic agriculture on biodiversity and abundance: a meta-analysis. Journal of Applied Ecology 42: 261-269.

Benton TG, Vickery JA \& Wilson JD. 2003. Farmland biodiversity: is habitat heterogeneity the key? Trend in ecology and evolution 18(4): 182-188.

Brussaard L, Caron P, Campbell B, Lipper L, Mainka S, Rabbinge R, Babin D \& Pullman M. 2010. Reconciling biodiversity conservation and food security: scientific challenges for a new agriculture. Current Opinion in Environmental Sustainability 2: 34-42.

Cabrera AL.1963-1970. Flora de la Provincia de Buenos Aires. Colección científica del INTA, Buenos Aires. Tomos I al V.

Cabrera AL \& Zardini EM.1978. Manual de la flora de los alrededores de Buenos Aires. Editorial ACME. 755 p.

Cerazo MB \& Conticello M. 2008. Comunidad de malezas en cultivos hortícolas de la ciudad de Neuquén (Argentina). Boletín de la Sociedad Argentina de Botánica 43(1-2): 121-135.

Clergue B, Amiaud FP, Lasserre-Joulin F \& Plantureux S. 2005. Biodiversity: function and assessment in agricultural areas. A review. Agronomy for Sustainable Development 25: $1-15$.

Comisión Nacional para el Conocimiento y Uso de la Biodiversidad (CONABIO). 2006-2012. Sitio de malezas de México. Herbario Virtual de CONABIO. Disponible en: http://www.conabio.gob.mx/otros/cgi-bin/herbario.cgi.

de Bello F, Lavorel S, Díaz S, Harrington R, Cornelissen J, Bardgett R, Berg M, Cipriotti P, Feld C, Hering D, Martins da Silva P, Potts S, Sandin L, Sousa J, Storkey J, Wardle D \& Harrison P. 2010. Towards an assessment of multiple ecosystem processes and services via functional traits. Biodiversity \& Conservation 19: 2873-2893.

de la Fuente EB, Suarez SA, Ghersa CM \& León RJC. 1999. Soybean weed comunities: relationships whit cultural history and crop yield. Agronomy Journal 91: 234-241.

de la Fuente EB, Suarez SA \& Ghersa CM. 2006. Soybean weed community composition and richness between 1995 and 2003 in the Rolling Pampas (Argentina). Agriculture, Ecosystems and Environment 115: 229-236.

di Castri F \& Younés T. 1996. Biodiversity, science and development: towards a new partnership. Wallingford, CAB International. $646 \mathrm{pp}$.

FAO. 2013. Stadistical yearbook 2013. World Food and Agriculture, FAO. Roma. 289 pp. Disponible en http://www.fao.org/docrep/018/i3107e/i3107e00.htm. Último acceso: diciembre de 2013. 
Fischer A \& Young JC. 2007. Understanding mental constructs of biodiversity: implications for biodiversity management and conservation. Biological Conservation: 136(2): 271-282.

Fischer J, Abson, DJ, Butsic V, Chappell MJ, Ekroos J, Hanspach, J \& Wehrden H. 2014. Land sparing versus land sharing: moving forward. Conservation Letters 7(3): 149-157.

Foley JA, DeFries R, Asner GP, Barford C, Bonan G, Carpenter R, Chapin FS, Coe MT, Daily GC, Gibbs HK, Helkowski JH, Holloway T, Howard EA, Kucharik CJ, Monfreda C, Patz JA, Prentice IC, Ramankutty N \& Zinder PK. 2005. Global Consequences of Land Use. Science 309: 570-574.

Gaba S, Reboud X \& Fried G. 2016. Agroecology and conservation of weed diversity in agricultural lands. Botany Letters 163(4): 351-354.

Gabriel D, Sait SM, Hodgson JA, Schmutz U, Kunin WE \& Benton TG. 2010. Scale matters: the impact of organic farming on biodiversity at different spatial scales. Ecology Letters 13(7): 858-869.

Garbach K, Milder JC, Montenegro M, Karp DS \& DeClerck FAJ. 2014. Biodiversity and Ecosystem Services in Agroecosystems. Encyclopedia of Agriculture and Food Systems 2: $21-40$.

Ghersa CM \& León RJC. 1999. Successional changes in agroecosystems of the Rolling Pampa. En: Walker LR (Ed.) Ecosystems of the World 16: Ecosystems of disturbed ground. Elsevier 20: 487-502.

Ghersa CM \& Martinez-Ghersa MA. 2000. Ecological correlates of weed seed size and persistence in the soil under different tilling systems: implications for weed management. Field Crops Research 67: 141-148.

Gliessman S. 2001. Agroecología. Processos ecológicos em agricultura sustentável. Segunda Edición. Editora da Universidade Río Grande do Sul, Brasil. 652 p.

Grime JP. 1979. Plant Strategies and Vegetation Processes. New York: John Wiley \& Sons. $222 \mathrm{p}$.

Guglielmini A, Ghersa C \& Satorre E. 2007. Coevolution of domesticated crops and associated weeds. Ecologia Austral 17: 167-178.

Halffer G, Morello J, Matteucci DS \& Solbrig OT. 1999. La biodiversidad y el uso de la tierra. En: Mateucci SD, Solbrig OT, Morello J, Halffter G (Eds.) Biodiversidad y uso de la tierra. Conceptos y ejemplos de Latinoamérica. Eudeba, Universidad de Buenos Aires.17-27 p.

Harlan JR. 1976. Las plantas y los animales que alimentan al hombre. En: Investigación y Ciencia, edición en español de Scientific American 65-74.

Henckel L, Borger L, Meiss, Gaba S \& Bretagnolle V. 2015. Organic field sustain weed metacommunity in farmland landscapes. Proceeding of de Royal Society B: 282: 20150002. 
Hernández MP \& Arambarri AN. 2011. Recursos fitoterapeuticos y comportamiento poblacional en dos sectores de la Ribera Rioplatense de Berisso, Buenos Aires, Argentina. BonPlandia 20(2): 137-148.

Herzon I \& Mikk M. 2007. Farmer's perceptions of biodiversity and their willingness to enhance it through agri-environment schemes: a comparative study from Stonia and Finland: Journal for Nature Conservation 15: 10-25.

Hole DG, Perkins AJ, Wilson JD, Alexander IH, Grice PV \& Evans AD. 2005. Does organic farming benefit biodiversity?. Biological Conservation 122: 113-130.

Kelemen E, Nguyen G, Gomerio T, Kovács E, Choisis JP, Choisis N, Paoletti M, Podmaniczky L, Rischawy J, Sarthou JP, Herzog F, Dennis P \& Bálaz K. 2013. Farmers' perceptions of biodiversity: lessons from a discourse-based deliberative valuation study Land Use Policy 35: 318-328.

Kross SM, Ingram KP, Long RF \& Niles MT. 2017. Farmer Perceptions and Behaviors Related to Wildlife and On-Farm Conservation Actions. Conservation Letters: 11(1): 1-9.

Hurrell JA, Delucchi G, Correa MN, Sánchez MI, Roitman G, Costantino FB, Ulibarri EA, Guaglianone ER \& Tur NM. 2009. Flora rioplatense: sistemática, ecología y etnobotánica de las plantas vasculares rioplatenses. Parte 3: Monocotiledóneas. Editorial LOLA, Buenos Aires. Vol. 4. 424 p.

Hyvonen T, Ketoja E, Salonen J, Jalli H \& Tiainen J. 2003. Weed species diversity and community composition in organic and conventional cropping of spring cereals. Agriculture, Ecosystems and Environment 97: 131-149.

Mace K, Munier-Jolain N \& Quere L. 2007. Time scales as a factor in decision-making by French farmers on weed management in annual crops. Agriculture Systems 93: 115-142.

Manhoudt AGE, Udo de Haes HA \& de Snoo GR. 2005. An indicator of plant species richness of semi-natural habitats and crops on arable farms. Agriculture, Ecosystems and Environment 109: 166-174.

Marshall EJP, Brown VK, Boatman ND, Lutman PJW \& Squire GR. 2002. The impact of herbicides on weed abundance and biodiversity. DEFRA, Informe PN0940.

Marshall EJP, Brown VK, Boatman ND \& Lutman PJW. 2003. The role of weeds in supporting biological diversity within crop fields. Weed Research 43: 77-89.

Martinez-Ghersa MA, Ghersa CM, Benech-Arnold R, Mac Donough R \& Sanchez R. 2000. Adaptative traits regulating dormancy and germination of invasive species. Plant species Biology 15: 127-137.

Marzocca A. 1976. Manual de malezas, 4ta edición actualizada por OJ Mársico y O. del Puerto. Hemisferio Sur SA, Buenos Aires. 564 p.

Marzocca A.1997. Vademécum de malezas medicinales de la Argentina indígena y exótica.: Orientación Gráfica Editora, Buenos Aires 363 p. 
Matteucci, SD \& Colma A. 1982. Metodología para el estudio de la vegetación. Monografía $\mathrm{n}^{0} 22$, Serie de biología de la OEA.

Molina AR (1998-1999) Malezas. Gráfica Corma, Buenos Aires. Vol. 1 y 2.

Mueller-Dombois D \& H Ellenberg.1974. Aims and Methods of Vegetation Ecology, Wiley, Nueva York, N Y. 547 p.

Petit S, Alignier A, Colbach N, Joannon A, Coeur D \& Thenail C. 2013. Weed dispersal by farming at various spatial scales. A review. Agronomy for Sustainable Development 33(1): 205-217.

Pimentel D, Stachow U, Takacs DA, Brubaker HW, Dumas AR, Meaney JJ, O'Neil JAS, Onsi DE \& Corzilius DB. 1992. Conserving biological diversity in agricultural/forestry systems. BioScience 42: 354-362.

Poggio S. 2004. Structure of weed communities occurring in monoculture and intercropping of field pea and barley. Agriculture, Ecosystems and Environment 19: 48-58.

Poggio S. 2005. Structure of weed communities occurring in monoculture and intercropping. Agriculture, Ecosystems, and Environment 19(1-2):48-58.

Poggio S. 2007. Relaciones entre la diversidad de especies vegetales y la heterogenidad espacial del paisaje agrícola pampeano. Tesis de Doctorado en Ciencias Agropecuarias. Facultad de Agronomía, Universidad de Buenos Aires, Argentina.125 p.

Poggio S. 2012. Cambios florísticos en comunidades de malezas: un marco conceptual basado en reglas de ensamblaje. Ecología Austral 22: 150- 158.

Poggio SL, Satorre EH \& de la Fuente EB. 2004. Structure of weed communities occurring in pea and wheat crops in the Rolling Pampa (Argentina). Agriculture, Ecosystem \& Environment 103: 225-235.

Radosevich SR, Holt J \& Ghersa CM. 2007. Ecology of weeds and invasive plants. Relationship to agriculture and natural resources management. 3ra edition. John Wiley \& Sons: Nueva York. 454 p.

Rahmamn G. 2011. Biodiversity and Organic farming: What do we know? Agriculture and Forestry Research 61(3): 189-208.

Rapoport EH, Marzocca A \& Drausal BS. 2009. Malezas comestibles del Cono Sur y otras partes del planeta. Ediciones Instituto Nacional de Tecnología Agropecuaria, Bariloche, Argentina. $216 \mathrm{p}$.

Saini E, Polack A. 2002. Enemigos naturales de los Trips sobre flores de malezas. Revista de Investigaciones Agroepecuarias 29(1): 117- 123.

Sarandón SJ \& Sarandón R. 1993. Un enfoque ecológico para una agricultura sustentable En: Goin F y C Goñi (Eds.) Bases para una política ambiental de la R. Argentina, Sección III, 19:279-286, HC Diputados de la Pcia de Buenos Aires. 
Scursoni J, Gigón R, Martín AN, Vigna M, Leguizamón ES, Istilart C \& López R. 2014. Changes in Weed Communities of Spring Wheat Crops of Buenos Aires Province of Argentina. Weed Science 62(1): 51-62.

Shepherd M, Pearce B, Cormack B, Phillipps L, Cuttle S, Bhogal A, Costigan P \& Unwin R. 2003. An assessment of the environmental impacts of organic farming. Una revision para Defra-funded project OF0405. ADAS Consultora Ltd. Mansfield. 80 pp. Disponible en: http://orgprints.org/6784/2/OF0405_909_TRP.pdf.

Soini K \& Akkula J. 2007. Framing the biodiversity of agricultural landscape: the essence of local conceptions and construction. Land Use Policy 24: 311-321.

Stilma ESC, Smit AB, Geerling-Eiff FA, Struik PC, Vosman B \& Korevaar H. 2009. Perception of biodiversity in arable production systems in the Netherlands. NJASWageningen Journal of Life Science 56: 391-404.

Stupino SA, Frangi JL, Sarandón SJ, Arturi MF \& Ferreira AC. 2008. Plant diversity in two farms under organic and conventional management in La Plata, Argentina. A case study. Revista Brasilera de Agroecología 3(3): 24-35.

Stupino SA, lermanó MJ, Gargoloff NA \& Bonicatto MM. 2014. La biodiversidad en los agroecosistemas. En: "Agroecología: Bases teóricas para el diseño y manejo de agroecosistemas sustentables", J. Sarandón \& C. Flores (Eds.). Colección de libros de cátedra, Secretaria de Asuntos Académicos-Edulp, UNLP. ISBN 978-950-34-1107-0.

Suárez SA, de la Fuente EB, Ghersa CM \& RJC León. 2001. Weed community as an indicator of summer crop yield and site quality. Agronomy Journal 93: 524-530.

Swift MJ, Izac MN \& van Noordwijk M. 2004. Biodiversity and ecosystem services in agricultural landscapes-are we asking the right questions? Agriculture, Ecosystems and Environment 104: 113-134.

Tscharntke T, Kleijn AM, Kruess A, Steffan- Dewenter I \& Thies C. 2005. Landscape perspectives on agricultural intensification and biodiversity-ecosystem service management. Ecology Letters 8: 857-874.

Tscharntke T, Clough Y, Wanger T, Jackson L, Motzke I, Perfecto I, Vandermeer J \& Whitbread A. 2012. Global food security, biodiversity conservation and the future of agricultural intensification. Biological Conservation 151(1): 53-59.

UNEP.1997. The Biodiversity Agenda. Decisions from the third Meeting of the Conference of the Parties to the Convention on Biological Diversity. 2da. ed., Buenos Aires, Argentina, Noviembre de 1996. $116 \mathrm{p}$.

Urban S. 2010. Synanthropic vegetation: pattern of varius disturbances on life history traits. Acta Botanica Croata 69(2): 215-227.

van Elsen T. 2000. Species diversity as a task for organic agriculture in Europe. Agriculture, Ecosystems and Environment 77: 101-109. 
Wilson R, Tucker M, Hooker HH, Lejeune JT \& Doohan D. 2008. Perceptions and bilief about weed management: perpectives of Ohio Grain and produce farmers. Weed Technology 22(2): 339-350.

Zanin G, Otto S, Riello L \& Borin M. 1997. Ecological interpretation of weed flora dynamics under different tillage systems. Agriculture, Ecosystems and Environment 66: 177-188. 


\section{CAPÍTULO 4}

\section{CARACTERIZACIÓN DE LOS TIPOS DE MANEJO DE LOS}

CULTIVOS HORTÍCOLAS EN LOS ALREDEDORES DE LA PLATA

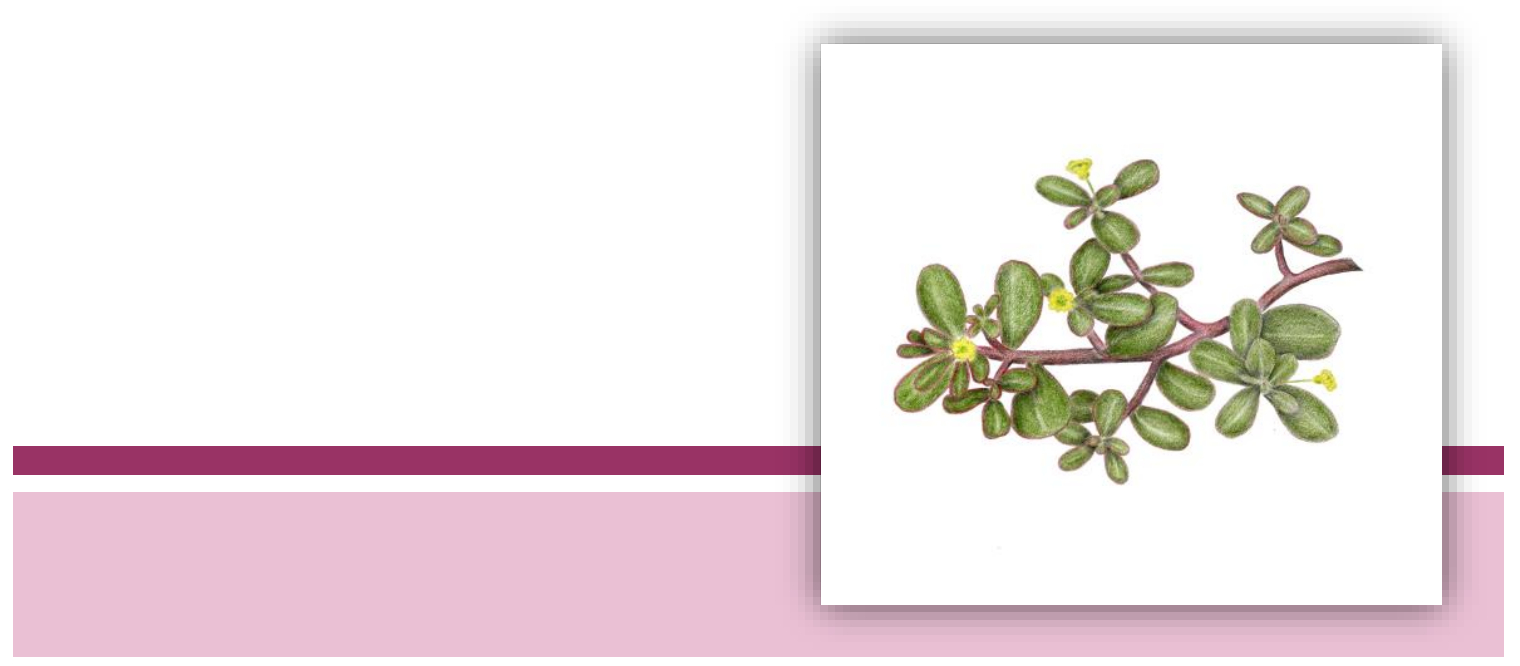




\section{CAPÍTULO 4}

Por lo menos una vez en la vida vas a necesitar un médico, un abogado, un arquitecto, pero todos los días, tres veces al día, vas a necesitar un agricultor.

Brenda Schoepp. Agricultora, escritora.

\section{CARACTERIZACIÓN DE LOS TIPOS DE MANEJO DE LOS CULTIVOS HORTÍCOLAS EN LOS ALREDEDORES DE LA PLATA}

\subsection{INTRODUCCIÓN}

El proceso de intensificación de la agricultura moderna refiere al incremento en el tiempo de utilización de una misma porción de tierra, con el objetivo de incrementar los rendimientos de los cultivos. Esto va asociado a un alto uso de insumos y a la especialización en el proceso productivo, con la consecuente simplificación de los agroecosistemas (Matson et al., 1997; de la Fuente \& Suárez, 2008). Estos procesos se asocian con la disminución de la diversidad de plantas espontáneas acompañantes de los cultivos. La pérdida de especies determina la pérdida de servicios ecológicos, que deben ser sustituidos por insumos externos para mantener los agroecosistemas funcionales (Altieri, 1999; Swift et al., 2004).

Sin embargo, los agroecosistemas no son manejados de la misma forma. Existen diferentes estilos de agricultura, entendidos como distintas formas de "hacer" $y$ "pensar" (Van der Ploeg, 1993). En el marco de una agricultura sustentable, se busca identificar las prácticas agrícolas que permitan mantener la biodiversidad en los agroecosistemas (Altieri, 1999; Sarandón, 2002; 2009; Schmitzberger et al., 2005). Por lo tanto, es necesario conocer y caracterizar adecuadamente los distintos estilos de agricultura para poder identificar las variables de manejo que podrían afectar o favorecer la diversidad.

Un alto impacto sobre la diversidad supone una alta intensidad de manejo. La intensidad de manejo representa, en esta tesis, un conjunto de prácticas de manejo cuantificables que describen la utilización de los insumos, de los recursos y el grado de simplificación de las fincas. Expresa el nivel de intensificación o grado de 
"artificialización" del agroecosistema estudiado, que tiene un posible efecto sobre diversidad de especies espontáneas.

El Cinturón Hortícola de La Plata, se caracteriza por el cultivo intensivo de productos para el consumo en fresco de la población urbana, bajo la modalidad de producción a campo (aire libre), invernáculo (bajo cubierta), o mixto (Benencia, 1994, 2009a).

En la últimas décadas, se han producido cambios en la región vinculados a fluctuaciones económicas y reconversiones tecnológicas constituyendo el cultivo protegido el símbolo del progreso técnico del período (Cieza, 2004; Barsky, 2005; García, 2010). Las tecnologías incorporadas tales como, la mecanización, variedades mejoradas, agroquímicos, y el riego localizado, han incrementado los rendimientos. Sin embargo, han tenido repercusiones tanto en el ambiente como en la forma de producción y comercialización. Actualmente se reconoce que el alto uso de agroquímicos y fertilizantes constituye uno de los problemas socio-ambientales más importantes de la horticultura bonaerense en general (Benencia \& Souza Casadinho, 1993; Bocero, 2002; Souza Casadinho \& Bocero, 2008; Alconada et al., 2011). En este sentido, se destaca la utilización de productos de amplio espectro como los herbicidas totales o, los "esterilizadores", de amplio efecto como plaguicidas que convierten al suelo en un sustrato inerte (MBTOC, 1994; Fields \& White, 2002). La composición de la producción ha sufrido variaciones pasando de una mayor diversificación a una especialización relativa en pocos cultivos sin rotaciones (Benencia, 1994; García, 2010).

Actualmente el Cinturón Hortícola presenta distintos estilos de agricultura. En virtud de esta complejidad suelen emplearse clasificaciones sobre la base de distintos criterios y objetivos. Una clasificación refiere a agricultores, fincas o manejos denominados convencionales y orgánicos. El término convencional indica a los sistemas "modernos" asociados con la Revolución Verde y su significado varía de acuerdo al objetivo de estudio (Hole et al., 2005). En esta tesis se considera como manejo convencional al caracterizado principalmente por el uso de productos químicos como fertilizantes, pesticidas y herbicidas (Bengsston et al., 2005). Pueden reconocerse "a priori" dos modalidades convencionales: 1- Convencional Intensivo (Cl), fincas altamente tecnificadas, a veces con una amplia superficie (hasta más de 20 ha), ocupada por pocos cultivos, un uso intensivo de la tierra y un alto uso de insumos químicos; 2Convencional de Bajos Insumos (CBI), con baja tecnificación, fincas de menor superficie

Diversidad Vegetal Espontánea en Agroecosistemas Hortícolas de La Plata y su relación con diferentes estilos de Agricultura: Importancia 
(generalmente inferior a 10 hectáreas), el uso de la tierra es intensivo y es limitado el uso de insumos químicos. Estas divisiones se ajustan a la clasificación de Benencia (1994) en productores empresariales y familiares medios, respectivamente.

Por otro lado, se observa un número menor pero creciente de fincas orgánicas (ORG) que buscan, generalmente, compatibilizar una producción de alimentos sanos minimizando el impacto sobre el ambiente. Las fincas oscilan entre 0,5 y 10 ha de superficie (Casadinho et al., 2009). Estos sistemas existen en Argentina desde 1992, reconocidos oficialmente por el IASCAV (Resolución de la SAGyP 423/92). Las normas en que se basan son las del IFOAM (International Federation Organic Agriculture Movement, 2007). Según esta normativa "se entiende por orgánico, a todo sistema de producción sustentable en el tiempo que mediante el manejo racional de los recursos naturales, sin la utilización de productos de síntesis química, brinde alimentos sanos y abundantes, mantenga o incremente la fertilidad del suelo y la diversidad biológica...". En esta tesis se considerarán agricultores, fincas o manejo orgánico a aquellos que cumplen con la norma del IFOAM indicada en el párrafo anterior. Ellos incluyen agricultores y fincas que han certificado su actividad a través de empresas habilitadas tal como lo exige la Resolución de la SAGyP 423/92, como también a aquellos que no han cumplido las instancias formales de certificación, y que venden sus productos a través de una relación de confianza con el consumidor o, bien los comercializan por otras vías sin identificarlos como orgánicos.

En otras regiones, como Europa o EEUU, la mayoría de los estudios comparativos de la diversidad clasifican las fincas en dos estilos contrastantes convencionales y orgánicos, diferenciados sólo por el uso o no de químicos. Sin embargo, no se consideran otras variables de manejo que podrían definir mejor las categorías (Bengtsson et. al., 2005). Dada la complejidad que presenta la horticultura platense, es esperable que además del uso de productos químicos, otras variables puedan resultar relevantes para caracterizar el manejo de la zona.

Diferentes autores han caracterizado las fincas hortícolas convencionales de la zona de acuerdo a sus características tecnológico-comerciales y de organización social del trabajo (Archenti et al., 1993; Cieza, 2004; Hang et al., 2010). A su vez, los autores han agrupado a los agricultores en la categoría empresarial o familiar (Benencia, 1994). En los distintos casos, las variables consideradas relacionadas con los insumos y

Diversidad Vegetal Espontánea en Agroecosistemas Hortícolas de La Plata y su relación con diferentes estilos de Agricultura: Importancia 
tecnologías se utilizaron como indicadoras del grado de tecnificación. Sin embargo, los estilos de agricultura mencionados ( $\mathrm{Cl}, \mathrm{CB}$ y ORG) no se han clasificado en función de variables que expresen el grado de intensidad de manejo. Si bien pueden establecerse categorías relativamente discretas de los distintos tipos de manejo ( $\mathrm{Cl}, \mathrm{BI}, \mathrm{ORG})$, las diferentes lógicas de producción y de prácticas empleadas por los agricultores permiten suponer que dichas categorías representan un gradiente de intensidad de manejo que va de los sistemas convencionales intensivos a los orgánicos. La caracterización del manejo hortícola permitirá establecer relaciones entre las fincas y las variables consideradas, determinando cuales son las diferencias y similitudes entre los grupos establecidos en cuanto a las variables de manejo más relevantes. Esto significa un aporte al conocimiento de la relación entre el manejo de los cultivos de la región con la diversidad de plantas espontáneas. Esta relación se analiza en los capítulos siguientes.

Dado los antecedentes, en este capitulo se apunta a caracterizar el manejo hortícola considerando criterios asociados a la superficie de las fincas, historia de uso de la tierra, partición y destino actual de los distintos cultivos, tipos de labores e insumos utilizados, partiendo de la preclasificación general citada. Dada la heterogeneidad que a priori se observa en el sector hortícola de la zona de estudio, podría plantearse que existe un gradiente de intensidad de manejo, donde los tipos orgánicos y convencionales intensivos representan los extremos.

El objetivo de este trabajo es formalizar la caracterización del manejo hortícola platense sobre la base de variables partiendo de una preclasificación en tres tipos (convencionales intensivos, bajos insumos y orgánicos) de fincas de la zona. Para ello se ponen a prueba las siguientes hipótesis:

Hipótesis 1:

En la zona estudiada, las fincas suelen ser encuadradas en dos categorías principales de manejo: orgánico y convencional. No obstante, las diferentes lógicas de producción y de prácticas empleadas por los agricultores son dependientes de múltiples factores, lo que permite esperar que las fincas integren un gradiente de intensidad de manejo.

Hipótesis 2:

Diversidad Vegetal Espontánea en Agroecosistemas Hortícolas de La Plata y su relación con diferentes estilos de Agricultura: Importancia 
Dada la heterogeneidad que presenta la horticultura platense, es esperable que además del uso de productos químicos, existan otras variables que puedan resultar relevantes para caracterizar el manejo de la zona.

\subsection{METODOLOGÍA}

\subsubsection{Selección de las fincas y realización de entrevistas}

Como se describió en el Capítulo 3, se seleccionaron 32 establecimientos de producción hortícola, comprendidos dentro de la región periurbana del Gran La Plata. Los mismos se situaron en las localidades de Pereyra, Lisandro Olmos, Los Hornos, Arana, El Pato, Colonia Urquiza, Etcheverry y Gorina (Figura 4.1). Las fincas visitadas presentaron diferentes situaciones de manejo calificadas "a priori" como convencionales intensivos $(\mathrm{Cl})$, convencionales de bajos insumos (CBI), y orgánicos (ORG), y comprendieron formas de cultivo bajo cubierta (invernáculo), a campo, o ambas formas de manejo (mixtos).

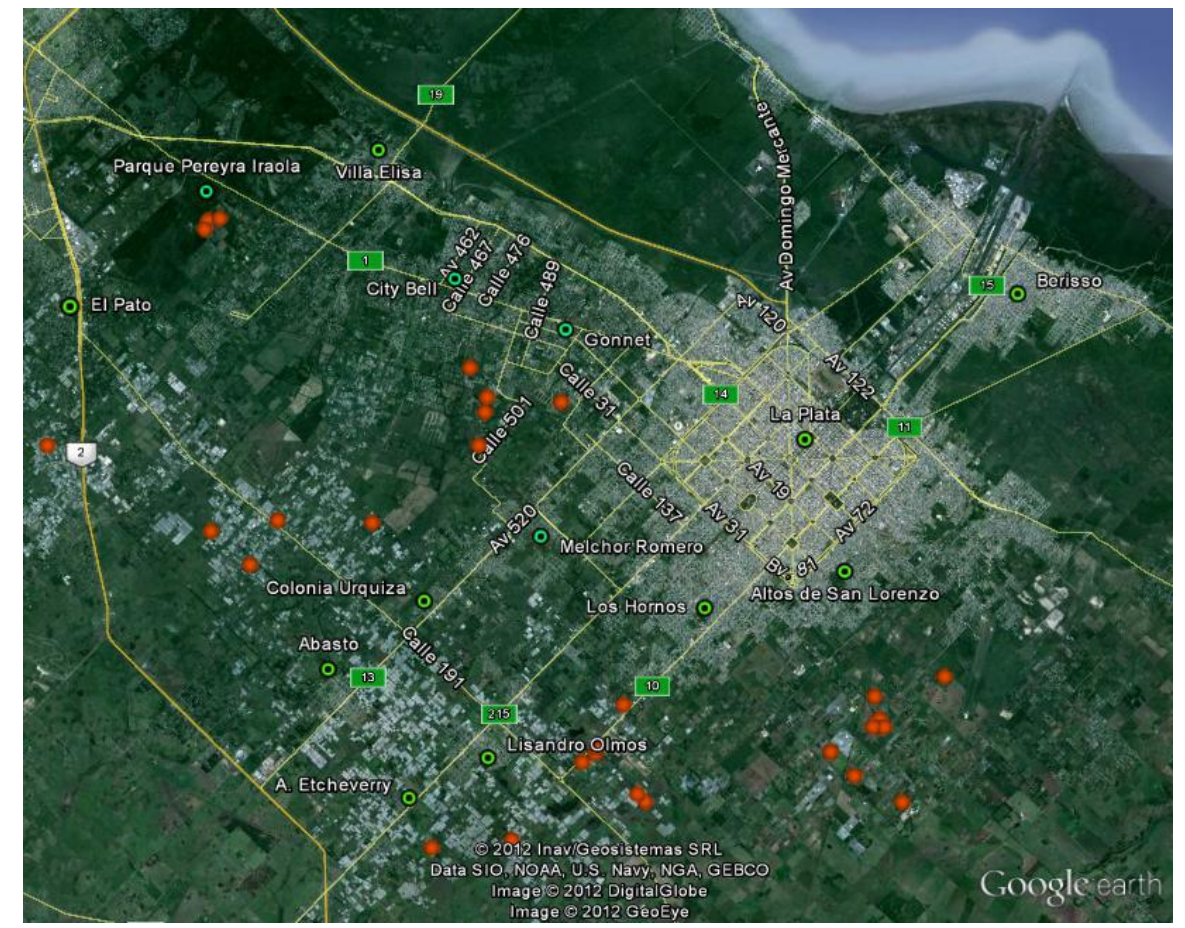

Figura 4.1. Distribución de las unidades de muestreo en el Cinturón Hortícola. Los círculos rojos indican las localizaciones geográficas de las fincas relevadas, los cuadrados verdes indican rutas provinciales y, los puntos verdes y negros las localidades citadas en el texto. Fuente: imagen de Google Hearh 2017. Modificado.

Diversidad Vegetal Espontánea en Agroecosistemas Hortícolas de La Plata y su relación con diferentes estilos de Agricultura: Importancia 
La encuesta social descripta en el Capítulo 3 consistió además en un conjunto de preguntas vinculadas con el presente capítulo. En este caso, se indagó acerca del manejo de los cultivos: laboreo del suelo, años en la producción, diversidad cultivada (especies de cultivo, policultivos), rotaciones, insumos e implementos utilizados para el control de la vegetación espontánea, fertilizantes y sistema de riego empleado que se detalló antes. En algunos casos, esta tarea requirió una segunda visita a las fincas.

\subsubsection{Cuantificación de las variables relevadas}

A partir de la información obtenida en las entrevistas, se cuantificaron las variables de manejo mediante índices que describen dichas variables, teniendo en cuenta la naturaleza distinta de las mismas (cuantitativas o cualitativas). Para la mayoría de las variables se construyeron previamente escalas ordinales. Las variables del manejo se seleccionaron considerando el posible efecto de las mismas sobre las plantas espontáneas. Por lo tanto, salvo los esterilizadores del suelo, no fueron incluidos en la entrevista los plaguicidas dirigidos a combatir otros organismos (insecticidas, fungicidas).

Los índices estuvieron integrados por una o dos componentes. Por ejemplo, el índice intensidad de laboreo comprendió el "tipo de herramientas utilizadas" junto con el "número de pasadas". Los valores resultantes de los índices expresaron el nivel de intensificación de acuerdo con el manejo característico en la horticultura platense (intensidad de manejo). En cada índice, el valor más alto representó el mayor grado de artificialidad o simplificación del sistema, el cual se corresponde con uso más intensivo del suelo y de los insumos (herbicidas, esterilizadores, fertilizantes), con una tendencia a la especialización en pocos cultivos, la ausencia de rotaciones y a una menor tolerancia a la presencia de espontáneas por parte del productor, respectivamente.

Descripción de los Índices:

En relación con la superficie de la finca, se cuantificaron dos variables: superficie cultivada total a campo y superficie cultivada total a invernáculo. Ambas variables se calcularon en cantidad de hectáreas cultivadas. Las mayores superficies totales y bajo invernáculo suelen relacionarse en la zona con agricultores tecnificados que tienen mayor acceso a los insumos, como herbicidas. Esto se relaciona indirectamente con el efecto sobre las espontáneas.

Diversidad Vegetal Espontánea en Agroecosistemas Hortícolas de La Plata y su relación con diferentes estilos de Agricultura: Importancia 
-Diversidad cultivada: se cuantificaron las variables riqueza total de cultivos ( $\mathrm{N}^{\circ}$ total de especies cultivadas al momento del muestreo). Las mismas variables se calcularon por hectárea cultivada.

-Historia de Uso de la finca: número de años que el actual agricultor tiene la finca bajo cultivo.

-Fertilizantes orgánicos: cantidad de Nitrógeno agregado en abonos (cama de pollo, cama de caballo, compost) por hectárea por año ( $\mathrm{Kg} \mathrm{N}$ ha- ${ }^{1}$ año- $\left.{ }^{-1}\right)$. Sólo se consideró este nutriente por su importancia en el desarrollo de las plantas, por ser el de uso más difundido en los agricultores. Asimismo, es el nutriente que se encuentra en mayor cantidad en los estiércoles (Salazar Sosa et al., 2003).

Para el cálculo se tuvo en cuenta el porcentaje de humedad del tipo de abono y el contenido porcentual de $\mathrm{N}$ total por peso seco de abono a partir de diferentes fuentes bibliográficas (Pascuali, 1980; Kupper, 2003; Del Pino et al., 2008; Oliveros et al., 2012).

-Fertilizantes químicos: se calculó la cantidad de Nitrógeno por hectárea por año (Kg N. ha- ${ }^{1}$.año- ${ }^{1}$ ) en fertilizantes de origen sintético de aplicación en el suelo. El fertilizante nitrogenado es el más incorporado en la agricultura convencional y puede modificar las relaciones de competencia con el cultivo y la vegetación espontánea (Tang et al., 2013).

-Esterilizadores: se consideró el uso de los desinfectantes del suelo o "esterilizadores" Bromuro de metilo o líquidos alternativos como el Metam sodio (de marca comercial Vapam); el primero, de uso más común en invernáculo. Se los diferenció de los herbicidas comunes debido a su amplio espectro de acción (MBTOC, 1994; Fields \& White, 2002). Se estableció un valor de 0 a aquella finca donde no se utilizaron esterilizadores y 1 si se emplearon esterilizadores.

-Rotaciones: representa el recambio de especies cultivadas en el tiempo. Se consideró la frecuencia de esta práctica, asignando en una escala ordinal el valor de 1 a la rotación frecuente de cultivos (cambio de cultivos consecutivos en la misma parcela), 2 con rotación esporádica de cultivos (rota los cultivos de manera irregular e infrecuente) y 3 sin rotación de los cultivos.

Diversidad Vegetal Espontánea en Agroecosistemas Hortícolas de La Plata y su relación con diferentes estilos de Agricultura: Importancia 
-Umbral de Malezas: expresa el nivel de malezas máximo o cobertura de plantas espontáneas tolerada por el agricultor, antes de que proceda a su control. Dicho término se homologó con el concepto de umbral de daño económico (Stern et al., 1959), de modo que un umbral de malezas alto significa una mayor tolerancia del agricultor a la presencia de dicha vegetación.

Se observó la densidad o cobertura de plantas espontáneas durante la recorrida de la finca. Dado que al momento de la observación, las distintas parcelas presentaban cultivos en distinta etapa de crecimiento y manejo, se determinó la cobertura de espontáneas observando parcelas que se encontraban desmalezadas y sin desmalezar. Esta información se contrastó con la percepción del agricultor detectada en la entrevista. Dado que el valor más alto del índice representa una mayor presión sobre las espontáneas, el valor máximo utilizado (3), significa una mayor intolerancia a las malezas o menor umbral. De esta forma se asigna el valor de 1 al nivel de malezas abundante (umbral alto), 2 a la condición de nivel escaso, únicamente en las etapas tempranas de desarrollo del cultivo y, 3 a la escasa presencia de malezas (bajo umbral de malezas).

-Riego: se consideró la utilización o no de algún método de riego suplementario, teniendo en cuenta la eficiencia en la aplicación del mismo y el grado de aprovechamiento del agua por las plantas. Se consideró que un sistema de riego eficiente es aquel donde el agua que se destina al cultivo es aprovechada en un porcentaje superior al 70\% (Tapia Contreras \& Osorio Ulloa, 1999). Esta condición se da en los sistemas donde el agua se conduce por tuberías, y se realiza en forma localizada sobre el cultivo como el riego por goteo, dejando menos disponibilidad para las plantas espontáneas. Un sistema menos eficiente, por el contrario, determina una mayor influencia sobre el ambiente y una mayor disponibilidad agua para las plantas espontáneas, como el riego por surco y aspersión. En base a esto, se estableció una escala ordinal donde el valor de 0 es la condición de secano, 1 el riego por goteo y, 2 el riego por surco o aspersión.

-Intensidad de uso del suelo: se refiere al grado de utilización de la misma porción de tierra en el tiempo. Mediante una escala se evaluó si el productor deja o no parcelas en descanso y cuál es el periodo en meses en que el suelo no es trabajado. A través de una escala ordinal, se asignó el valor de 0 si el suelo descansa más de 6 meses, 1 para

Diversidad Vegetal Espontánea en Agroecosistemas Hortícolas de La Plata y su relación con diferentes estilos de Agricultura: Importancia 
un descanso entre 3 y 6 meses, 2 hasta 3 meses de descanso y 3 si no se realiza descanso.

-Comercialización: se incluyó como variable de manejo debido que el sector productivo constituye el primer eslabón en el sistema de producción-comercialización (Bocchicchio y Cattáneo, 2009) y el agricultor es quien decide que productos emplear de acuerdo a sus posibilidades de comercialización. La variedad de cultivos seleccionada podría tener influencia sobre la presencia de especies espontáneas en las fincas. Se consideraron las formas de comercialización mayorista y minorista, esta última dividida en 2 categorías. En base a esto, se construyó una escala donde se asignó un valor de $1 \mathrm{si}$ se comporta como comerciante minorista vendiendo directamente al consumidor (ferias, venta domiciliaria), 2 si el agricultor vende a un minorista (consignatario, supermercado, verdulería) y, 3 si vende a un mercado mayorista. Dado que en una misma finca se podía presentar más de una modalidad de venta, se consideró como destino comercial principal aquella categoría que representa el $80 \%$ del volumen de producción de la finca.

-Control químico de malezas: indica el grado de mortandad o agresividad del herbicida sobre la vegetación espontánea. Se construyó una escala que expresó el tipo de herbicida en cuanto a la selectividad y forma de aplicación, de acuerdo a la guía de productos fitosanitarios de la Cámara de Sanidad Agropecuaria y fertilizantes CASAFE (2002). Las formas totales y sistémicas son las que tienen un mayor efecto de mortalidad sobre las espontáneas. Herbicida total significa que matan tanto a plantas dicotiledóneas como a monocotiledóneas, y sistémico refiere a que tiene efecto sobre todas las partes de la planta (raíz, tallo y hojas). Todos los herbicidas selectivos y sistémicos resultaron ser exclusivamente residuales, por lo que se descartó la residualidad como otra categoría a considerar. Además los agricultores manifestaron en las entrevistas aplicar los productos según marbete, por lo tanto, las dosis no fueron consideradas en el índice. Se le asignó el valor de 0 a la no aplicación de herbicidas, 1 al uso de herbicidas selectivos, 2 a los totales y de contacto y, 3 a los totales y sistémicos. Por otro lado, se cuantificó el número de aplicaciones por lo que el índice resultante fue: 
-Control químico de malezas $=\Sigma$ (categoría del herbicida * el número de aplicaciones por ciclo del cultivo), donde la categoría del herbicida es el valor asignado al tipo de herbicida en la escala ordinal.

En esta fórmula los herbicidas aplicados abarcan toda la superficie considerada (a campo o invernáculo). En algunos casos herbicidas diferentes se aplican en distintos cultivos o grupos por tipo morfológico (cultivos de raíz u hoja) que implican una partición de la superficie total. Por lo tanto, para no sobrestimar el valor del indicador obtenido, se lo dividió por el número total de herbicidas diferentes donde cada producto se aplica exclusivamente en uno o más cultivos o tipos morfológicos:

Control químico de malezas $=\Sigma$ (Categoría del herbicida * el número de aplicaciones por ciclo de cultivo) / número de herbicidas diferentes.

-Control físico de malezas: se consideraron las maquinarias que se utilizan para desmalezar como las que además se utilizan para el laboreo del suelo y, el número de pasadas. También se incluyeron las herramientas manuales y el número de carpidas. Se tuvo en cuenta el grado de control en cuanto la superficie abarcada (lomo, surco, ambos). Se construyó una escala en la que se asignó el valor 1 al uso de herramientas como escardillo, carpidor y/o rastra de dientes, 2 al uso de azada y/o zapín y, 3 a la aporcadora y/o rastra de discos. El índice resultante fue:

Control físico de malezas $=\Sigma$ (Categoría de la herramienta * el número de pasadas). Donde la categoría es el valor obtenido en la escala ordinal de las herramientas.

-Intensidad de laboreo del suelo: para establecer la intensidad de laboreo se consideró la maquinaria utilizada en la preparación primaria del suelo (arada) y en la preparación de la cama de siembra (labranza secundaria) y, el número de pasadas del implemento. Se construyó una escala a partir de la cual se la asignó el valor de 0 al uso de cultivador, vibrocultivador o rastra de dientes, 1 al uso de cincel, cultivador, arado de disco, o rastra de disco y 2 , arado de reja y rotovacter. Por lo tanto, el índice resultó ser:

Intensidad de laboreo del suelo $=\sum$ (Categoría de la herramienta * el número de pasadas).

Diversidad Vegetal Espontánea en Agroecosistemas Hortícolas de La Plata y su relación con diferentes estilos de Agricultura: Importancia para la sustentabilidad 


\subsubsection{Análisis de los datos}

Se ordenaron las fincas en función de las variables de manejo. Para ello se utilizaron los valores obtenidos de los índices descriptos anteriormente. Como el manejo a campo suele presentar diferencias respecto del invernáculo, se analizaron grupos de variables separadamente para ambas modalidades. El ordenamiento se realizó mediante un análisis de Componentes Principales con el empleo del programa MVSP 3.1 (Digby \& Kempton, 1987). Es un método multivariado de síntesis de información, que consiste en reducir la dimensión de un conjunto de variables observadas (originales) posiblemente correlacionadas a un número más pequeño (componentes principales), que son combinaciones lineares de las variables de partida, no correlacionadas entre sí y que explican la mayor variabilidad total. Busca la proyección según la cual los datos quedan mejor representados en términos de mínimos cuadrados. Cuanto mayor sea su varianza mayor es la cantidad de información que lleva incorporada dicha componente. Con la reducción de la dimensión se simplifica la dispersión de las observaciones poniéndose en evidencia posibles agrupamientos y además, permite detectar las principales variables responsables de la dispersión y analizar relaciones entre ellas.

Atendiendo a las diferencias entre variables y rango de valores de los índices, la matriz de datos fue estandarizada a media 0 y varianza 1.

Se analizaron los motivos por los cuales los agricultores deciden cultivar determinado grupo de especies. Para cada opción manifestada por el conjunto de agricultores, se calculó el porcentaje de aquellos que expresaron dicha opción, pudiendo el mismo agricultor señalar más de una.

\subsection{RESULTADOS}

\subsubsection{Características generales del manejo hortícola platense}

Las fincas tuvieron en promedio una superficie total de 9,96 ha $( \pm 11)$ y una superficie cultivada de 6,8 ha $( \pm 8,4)$. La superficie promedio cultivada a campo fue de 5,5 ha con un rango de variación entre 0,8 y 38 ha. La superficie promedio cultivada bajo cubierta fue de 2,4 ha variado entre 0,05 y 10 ha.

Diversidad Vegetal Espontánea en Agroecosistemas Hortícolas de La Plata y su relación con diferentes estilos de Agricultura: Importancia 
La riqueza de cultivos encontrada al momento del muestreo varió entre 2 y 29 especies de cultivo, con un máximo de 10 cultivos por hectárea. Los principales motivos que los agricultores manifestaron acerca de la elección de sus cultivos, fueron: porque son los de mayor rentabilidad (38\%); porque los demanda el canal de comercialización al que está vinculado el agricultor (34\%); por su facilidad de manejo $(31 \%)$ y porque son los que saben cultivar (20\%).

Superficie a campo (Tabla 4.1). Las herramientas utilizadas para el laboreo del suelo fueron, en distinta combinación y formas de manejo, las de alto y medio impacto como el arado de reja, rastra de discos y cincel. El uso de rotovacter (de alto impacto) fue más generalizado en los cultivos de manejos intensivos. En todos los manejos, los fertilizantes químicos más difundidos fueron la urea y el nitrato de amonio y, como abono orgánico, el estiércol de gallina. Además, el uso de compost fue exclusivo de los cultivos orgánicos. El uso de riego por surco predominó en todos los tipos de manejo. Para el manejo de malezas se utilizaron azada, zapín y escardillo, destacándose en los orgánicos el uso de zapín y el desmalezado a mano (modalidades de control más selectivas). Los herbicidas más utilizados en los cultivos convencionales intensivos fueron el metribuzin (de marca comercial Sencorex), selectivo de amplio espectro y sistémico y, el herbicida paraquat (Gramoxone), total y de contacto. En las fincas de bajos insumos, del total de herbicidas empleados, se utilizaron cerca de la mitad de productos comerciales ( 5 vs 9 ) y en menor número de agricultores en comparación con los intensivos.

Superficie de invernáculo (Tabla 4.2). El laboreo del suelo fue diverso en cuanto al tipo de herramientas utilizadas en los distintos manejos, destacándose el uso de cincel en cultivos orgánicos y el uso, además, de rotovacter en los intensivos. El uso de fertilizantes químicos fue más frecuente en los cultivos intensivos, principalmente nitrato de potasio y el uso de los abonos orgánicos fue más frecuente en los otros manejos. En el invernáculo, dominó el riego por goteo. El 84 \% de las fincas intensivas utilizó un control químico de espontáneas con esterilizadores (Bromuro de Metilo o Vapam) y, en menor medida, con herbicidas totales (Gramoxone). Los agricultores de bajos insumos utilizaron en invernáculo solamente herbicidas. En el manejo de malezas las herramientas más utilizadas fueron las mismas que en el manejo a campo.

Diversidad Vegetal Espontánea en Agroecosistemas Hortícolas de La Plata y su relación con diferentes estilos de Agricultura: Importancia 
Tabla 4.1. Descripción del principal manejo realizado a campo en las fincas hortícolas del Gran La Plata. Los valores indican el porcentaje de agricultores por manejo (columnas) que utilizan cada insumo o herramienta (filas). En la mayoría de los casos utilizan más de un insumo o herramienta por rubro.

\begin{tabular}{|c|c|c|c|}
\hline & Intensivo & Bajos Insumos & Orgánico \\
\hline \multicolumn{4}{|l|}{ Laboreo del suelo } \\
\hline Arado de reja & 36 & 78 & 50 \\
\hline Arado de disco & 29 & 67 & 67 \\
\hline Cincel & 43 & 22 & 50 \\
\hline Rotovacter & 93 & 22 & 33 \\
\hline Rastra de disco & 14 & 11 & 17 \\
\hline Vibrocultivador & 21 & 0 & 50 \\
\hline Rastra de dientes & 14 & 11 & 0 \\
\hline \multicolumn{4}{|l|}{ Fertilizantes } \\
\hline \multicolumn{4}{|l|}{ Químicos } \\
\hline Urea & 43 & 44 & 0 \\
\hline Nitrato de Potasio & 7 & 0 & 0 \\
\hline Nitrato de Amonio & 36 & 22 & 0 \\
\hline Complejo NPK & 21 & 22 & 0 \\
\hline Fosfato diamónico & 14 & 11 & 0 \\
\hline \multicolumn{4}{|l|}{ Abonos } \\
\hline Cama de pollo & 29 & 0 & 0 \\
\hline Cama de caballo & 21 & 0 & 0 \\
\hline Estiércol de conejo & 0 & 11 & 0 \\
\hline Estiércol de gallina & 57 & 56 & 50 \\
\hline Compost & 0 & 0 & 17 \\
\hline \multicolumn{4}{|l|}{ Riego } \\
\hline Surco & 93 & 56 & 67 \\
\hline Aspersión & 7 & 44 & 17 \\
\hline Goteo & 0 & 0 & 17 \\
\hline \multicolumn{4}{|l|}{ Manejo de malezas } \\
\hline \multicolumn{4}{|l|}{ Físico } \\
\hline Desmalezadora & 14 & 0 & 0 \\
\hline Aporcadora & 0 & 33 & 0 \\
\hline Arado de reja & 14 & 33 & 0 \\
\hline Rastra de disco & 7 & 0 & 0 \\
\hline Escardillo & 64 & 56 & 33 \\
\hline Azada & 57 & 56 & 50 \\
\hline Zapin & 57 & 33 & 83 \\
\hline Arrancada a mano & 0 & 0 & 17 \\
\hline Herbicidas & & & \\
\hline
\end{tabular}




\section{Capítulo 4}

\begin{tabular}{lccc} 
Paraquat & 42 & 11 & 0 \\
Gesagard & 21 & 0 & 0 \\
Trifuralina & 14 & 0 & 0 \\
Metribuzin & 43 & 11 & 0 \\
S-metacloro & 29 & 11 & 0 \\
Atrazina & 7 & 0 & 0 \\
Glifosato & 21 & 22 & 0 \\
Lenacil & 7 & 0 & 0 \\
Prometrina & 7 & 11 & 0 \\
\hline
\end{tabular}


Tabla 4.2. Descripción del principal manejo realizado bajo cubierta en fincas hortícolas del Gran La Plata. Los valores indican el porcentaje de productores por manejo (columnas) que utilizan un insumo o herramienta (filas). En la mayoría de los casos utilizan más de un insumo o herramienta por rubro.

\begin{tabular}{|c|c|c|c|}
\hline & Intensivo & Bajos Insumos & Orgánico \\
\hline \multicolumn{4}{|l|}{ Laboreo del suelo } \\
\hline Arado de reja & 0 & 50 & 0 \\
\hline Arado de disco & 8 & 50 & 25 \\
\hline Cincel & 85 & 0 & 100 \\
\hline Rotovacter & 92 & 0 & 50 \\
\hline Rastra de disco & 15 & 0 & 50 \\
\hline Vibrocultivador & 15 & 0 & 25 \\
\hline \multicolumn{4}{|l|}{ Fertilizantes } \\
\hline \multicolumn{4}{|l|}{ Químicos } \\
\hline Urea & 15 & 0 & 0 \\
\hline Nitrato de potasio & 38 & 0 & 0 \\
\hline Ácido nítrico & 15 & 0 & 0 \\
\hline Nitrato de amonio & 8 & 0 & 0 \\
\hline Complejo NPK & 0 & 50 & 0 \\
\hline \multicolumn{4}{|l|}{ Orgánicos } \\
\hline Cama de pollo & 23 & 50 & 50 \\
\hline Cama de caballo & 23 & 50 & 0 \\
\hline Estiércol de gallina & 23 & 50 & 25 \\
\hline Compost & 0 & 0 & 25 \\
\hline \multicolumn{4}{|l|}{ Riego } \\
\hline Surco & 8 & 50 & 0 \\
\hline Goteo & 92 & 50 & 100 \\
\hline \multicolumn{4}{|l|}{ Manejo de malezas } \\
\hline \multicolumn{4}{|l|}{ Físico } \\
\hline Escardillo & 8 & 0 & 0 \\
\hline Azada & 15 & 50 & 25 \\
\hline Zapín & 23 & 100 & 100 \\
\hline Arrancada a mano & 0 & 0 & 25 \\
\hline \multicolumn{4}{|l|}{ Herbicidas } \\
\hline Gramoxone & 23 & 0 & 0 \\
\hline Glifosato & 8 & 100 & 0 \\
\hline S-metacloro & 0 & 100 & 0 \\
\hline \multicolumn{4}{|l|}{ Esterilizadores } \\
\hline Vapam & 23 & 0 & 0 \\
\hline Bromuro de metilo & 61 & 0 & 0 \\
\hline
\end{tabular}

Diversidad Vegetal Espontánea en Agroecosistemas Hortícolas de La Plata y su relación con diferentes estilos de Agricultura: Importancia 


\subsubsection{Caracterización de las fincas a partir del método de ordenación}

En el análisis de la distribución de las fincas en función de variables de manejo, las tres primeras Componentes Principales (CP) sumadas explicaron cerca del $60 \%$ de la variabilidad (Tabla 4.3). Las componentes CP1 y CP2 explicaron sumadas el $46 \%$ de la variación. En la Figura 4.2 se observan los resultados en las primeras dos componentes. En el lado positivo de la primer componente $(\mathrm{CP} 1+)$, las variables que más se asociaron (flechas más cercanas al eje $x$ positivo) fueron: el menor umbral de malezas o cobertura de espontáneas tolerada por el agricultor, la mayor tendencia a la ausencia de rotaciones, la mayor intensidad en el uso del suelo, el empleo de los esterilizadores, la mayor historia de uso y el incremento en la cantidad utilizada de fertilizantes químicos, y la tendencia a la comercializar los productos en un mercado mayorista. En el sentido negativo, opuesto a la anterior (CP1-), se observó un incremento en el número (riqueza) de cultivos. En CP2 las variables que más se asociaron al eje se orientaron en el sentido negativo (CP2-) y fueron el mayor desmalezado químico, la aplicación de un riego más intensivo y el incremento en la superficie cultivada. En C3 las variables que más se asociaron al eje fueron el incremento del desmalezado físico y el aumento de la fertilización orgánica, de sentido opuesto entre sí (Tabla 4.3).

La comercialización fue significativa para los tres ejes, sin embargo, se la consideró más asociada a la $\mathrm{C} 1$, dada la proporción que explica en relación a la variabilidad total del eje. A su vez, se asoció negativamente a la diversidad cultivada. 
Tabla 4.3. Resultados del análisis en componentes principales (ACP) para los tres primeros ejes (CP). El autovalor (eigenvalue) es el valor propio de cada componente principal, y justo debajo la proporción de varianza explicada por cada una de ellos y la varianza explicada acumulada. Los datos de varianza explicada permiten establecer cuántos componentes principales se van a utilizar en el análisis. Los números en negrita representan los valores de correlación significativos de las variables (peso de las variables), para cada componente.

\begin{tabular}{lccc}
\hline & CP1 & CP2 & CP3 \\
\hline Autovalor & 3.987 & 2.448 & 1.512 \\
Porcentaje & 28.482 & 17.485 & 10.801 \\
Porcentaje acumulado & 28.482 & 45.967 & 56.768 \\
& \multicolumn{2}{c}{ Peso de las variables } & \\
Umbral de malezas & $\mathbf{0 . 3 8 5}$ & -0.084 & -0.081 \\
Ausencia de rotaciones & $\mathbf{0 . 3 8 2}$ & 0.052 & 0.125 \\
Intensidad de uso del suelo & $\mathbf{0 . 3 0 0}$ & 0.142 & -0.180 \\
Esterilizadores & $\mathbf{0 . 3 6 3}$ & 0.264 & 0.096 \\
Historia de uso & $\mathbf{0 . 2 9 6}$ & -0.226 & -0.102 \\
Fertilización química & $\mathbf{0 . 2 7 8}$ & $\mathbf{- 0 . 2 9 0}$ & -0.254 \\
Comercialización & $\mathbf{0 . 2 7 4}$ & -0.294 & 0.287 \\
Riqueza cultivada & $\mathbf{0 . 3 5 8}$ & 0.037 & -0.213 \\
Desmalezado químico & 0.133 & $-\mathbf{0 . 4 5 5}$ & -0.104 \\
Riego & -0.259 & $\mathbf{- 0 . 4 2 3}$ & 0.287 \\
Superficie cultivada & -0.072 & $\mathbf{- 0 . 4 0 3}$ & -0.044 \\
Intensidad de laboreo & 0.020 & -0.233 & 0.298 \\
Desmalezado físico & -0.150 & -0.212 & $\mathbf{0 . 5 5 1}$ \\
Fertilización orgánica & -0.056 & -0.172 & $\mathbf{- 0 . 5 7 3}$ \\
& & & \\
\hline
\end{tabular}

Las fincas se distribuyeron principalmente a lo largo de la CP1 de acuerdo a un gradiente de intensidad de manejo (Figura 4.2) 


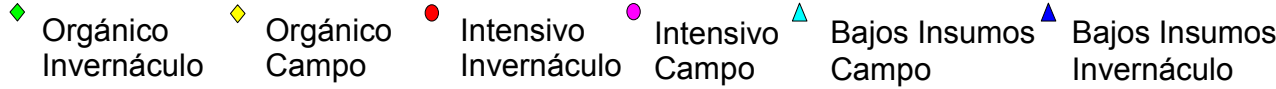

CP $2(+)$

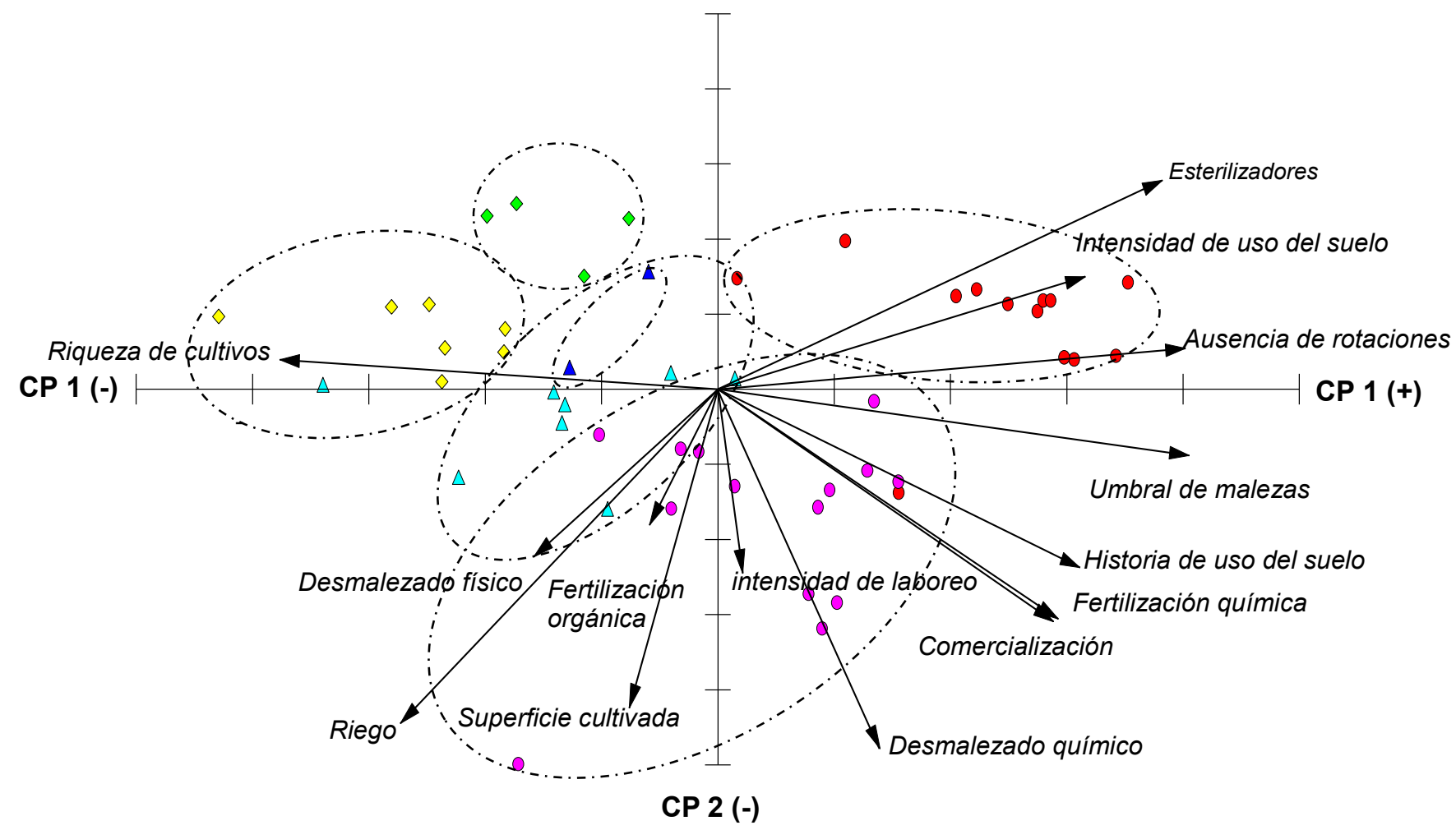

Figura 4.2. Ordenamiento en componentes principales (ACP) en 2 dimensiones (CP1 y CP2) de las fincas hortícolas de la Plata en función de las variables de manejo más importantes. Los símbolos geométricos similares representan fincas con el mismo tipo de manejo; las elipses delimitan las fincas con similar tipo de manejo. Las flechas (vectores) representan cada variable y su longitud el grado de importancia.

En el plano CP1 (+)_CP2 (+ -) se observó un grupo de fincas convencionales intensivas que se caracterizaron por presentar la mayor antigüedad en la producción (entre los 30 y 50 años) y el cultivo de pocas especies de hortalizas y verduras sumado a un uso intensivo de la tierra. Estas fincas presentaron además, un alto uso de insumos químicos, ya sean herbicidas, y/o fertilizantes (en promedio $631 \mathrm{KgN}$.ha- ${ }^{1}$.año- ${ }^{-1}$ de abonos orgánicos; $243 \mathrm{KgN}$.ha- ${ }^{1}$.año- ${ }^{1}$ de fertilizantes sintéticos, con un valor máximo de $450 \mathrm{KgN}$.ha- ${ }^{1}$.año- ${ }^{1}$ ). El manejo $\mathrm{Cl}$ bajo invernáculo se diferenció del $\mathrm{Cl}$ a campo por una mayor tendencia al monocultivo continuo (es decir sin rotaciones y sin incluir períodos de descanso del suelo), por el control de espontáneas fundamentalmente por 
esterilizadores y por el riego por goteo. En cambio, el Cl a campo se caracterizó tener una mayor superficie cultivada, por el uso de riego por aspersión o surco y por la mayor aplicación de herbicidas.

En el plano CP1 (-)_CP2 (+) se observó que las fincas orgánicas se caracterizaron por una mayor diversidad de cultivos espacial y temporalmente, sin uso de fertilizantes/herbicidas sintéticos, una mayor presencia de plantas espontáneas toleradas por el agricultor y con tendencia a canales de comercialización diversificados El uso de fertilizantes orgánicos fue, en promedio, de $670 \mathrm{KgN}$. ha- ${ }^{-1}$.año- ${ }^{-1}$. Se observó, además, que las fincas ORG comparadas con las intensivas, presentaron una mayor similitud entre el manejo a campo y en invernáculo, con algo más de especies cultivadas en el campo. Las fincas de bajos insumos tuvieron una mayor dispersión, presentando características intermedias con los otros grupos (posicionadas en el medio del gradiente). La diversidad cultivada encontrada en las mismas las vinculó con las fincas orgánicas, y se destacaron por un uso intermedio de insumos. En relación a esto último, se observó en este grupo que el uso de fertilizantes orgánicos (200 KgN .ha- ${ }^{-}$.año- $\left.{ }^{1}\right)$ y sintéticos (160 KgN .ha- ${ }^{1}$.año- ${ }^{-}$) fue menor que los intensivos, y predominó el control mecánico de espontáneas por sobre el químico.

En general, las fincas se distribuyeron a lo largo del gradiente conformando grupos relativamente definidos de similares características. La forma de manejo del grupo al que perteneció cada finca como resultado de la ordenación, coincidió con la categoría asignada "a priori" a cada finca en el momento de la selección de las mismas. No obstante, un número mayor de variables cuantificadas explicaron las categorías.

\subsection{DISCUSIÓN}

Los resultados confirman la existencia de un gradiente de intensidad de manejo en las fincas del Cinturón Hortícola de La Plata. Dicho gradiente permite diferenciar, como grupos relativamente definidos, a aquellos que representan los extremos del espectro analizado: convencionales intensivos y orgánicos.

El alto número de variables significativas encontradas sugieren la obtención de una caracterización más precisa de las fincas por el tipo de manejo y forma de producción (campo e invernáculo) (Bengtsson, 2005). Esto indica que no es solo el uso de químicos 
lo que diferencia a los manejos convencionales intensivos de los orgánicos, confirmando la segunda hipótesis.

Las superficies promedio y máximas cultivadas a campo e invernáculo resultaron cercanas a los valores encontrados en un estudio del Cinturón Hortícola $(n=100)$ (Hang, et al. 2010). Los resultados muestran que las fincas seleccionadas fueron representativas de la zona, en lo que respecta a la superficie productiva utilizada y el manejo realizado.

Como se observó en CP1 $(+)$, las fincas convencionales intensivas se caracterizaron por un uso más intensivo de la tierra y una menor diversidad cultivada. Esta simplificación del agroecosistema se asoció al alto uso de insumos químicos, principalmente fertilizantes sintéticos y herbicidas de amplio espectro. Considerando este último aspecto, se observó el uso de productos químicos como los esterilizadores del suelo (Bromuro de metilo) o el uso de herbicidas totales (Gramoxone). A su vez, la baja cobertura de espontáneas tolerada caracterizó a estos agricultores, lo que sugiere una estrecha relación entre la baja tolerancia del agricultor a las malezas y un control químico más intensivo.

Las características señaladas para el manejo convencional intensivo, coincidieron con la tipología de agricultores empresariales de acuerdo a la clasificación de Benencia et al. (2009a): agricultores capitalizados que hacen un uso intensivo del recurso, llegando a utilizar más de dos veces en el año la misma superficie lo que se logra con la aplicación constante de agroquímicos y maquinaria adecuada. La mayor antigüedad en el tipo de producción registrada (entre los 30 y 50 años de producción) se debió a que los agricultores siguieron una tradición familiar, manejando las fincas que antiguamente eran manejadas por sus antecesores y de los cuales aprendieron la actividad.

La tendencia a producir pocas especies cultivadas, una de las amenazas de la biodiversidad, tendría una causa principal asociada al destino de la comercialización que fue principalmente mayorista, sistema que requiere mayores volúmenes de producción por cultivo. 
Las diferencias encontradas entre el manejo convencional en el invernáculo respecto del campo coinciden con otros autores (Hang et al., 2010) e indican que el manejo del invernáculo convencional representa la forma más extrema de intensidad y especialización en el gradiente hortícola. Esto se corresponde con el objetivo de la producción bajo cubierta que es lograr una mayor productividad evitando las inclemencias climáticas. Al "mejorar" las condiciones para el cultivo, también se vuelven más favorables las condiciones ambientales para el desarrollo de otros organismos, por lo que se requiere un mayor control sobre las adversidades. Estos aspectos conducen a la especialización en el tipo de producción a uno o pocos cultivos más rentables, con el empleo de tecnologías asociadas (riego por goteo, equipo para aplicación de bromuros, ferti-irrigación, control de la atmósfera interior, etc.) (Benencia et al., 1992; Benencia et al., 2009a) acordes con una utilización de los recursos y un control de adversidades más intensivos. Por lo tanto, el cultivo bajo cubierta implica la adopción de un paquete tecnológico cerrado. La repetición del mismo cultivo en el tiempo y sin descanso del suelo se relacionó con los costos de producción que implica esta tecnología, dado que se requiere obtener una mayor productividad para que sea rentable (Benencia et al., 1992). A su vez, la tendencia al monocultivo continuo estaría en relación con la comercialización, donde el agricultor empresarial puede tener posibilidades de dominar las instancias principales del mercado, a través de la obtención de un puesto en el mercado mayorista, o la búsqueda de estrategias que lo diferencien económicamente, como es la obtención de grandes volúmenes de un producto de alta calidad (Garcia \& Hang, 2007). Esto coincide con los resultados de Hang et al. (2010) quienes definieron a los agricultores medios-grandes con predominio de producción bajo cubierta como altamente capitalizados, donde la vía de comercialización son los mercados concentradores.

El manejo convencional intensivo a campo presentó una mayor variabilidad que el manejo en invernáculo, destacándose fincas de mayor superficie cultivada, en concordancia con la mayor superficie promedio observada en el campo respecto del invernáculo. Además, se caracterizó por el control efectivo de malezas por medio de herbicidas totales y el uso de riego por surco.

Las fincas orgánicas constituyeron otro grupo definido dentro del gradiente y, a diferencia de las Intensivas, presentaron una mayor diversidad y rotación de cultivos, períodos de descanso del suelo, sin utilización de productos químicos sintéticos

Diversidad Vegetal Espontánea en Agroecosistemas Hortícolas de La Plata y su relación con diferentes estilos de Agricultura: Importancia 
(herbicidas y/o fertilizantes). Un rasgo destacable fue la mayor tolerancia de los agricultores orgánicos a la presencia de vegetación espontánea (umbral alto de malezas), en contraposición con convencionales intensivos. Esto se correspondió, en parte, con las formas de control no químicas y más selectivas sobre algunas especies, tal como se observó en el predominio del uso de zapin y el desmalezado a mano.

La mayor diversidad de cultivos podría atribuirse a que el agricultor tiene, muchas veces, una lógica que busca diversificar el sistema para favorecer procesos ecológicos como la regulación biótica y el ciclado de nutrientes. No obstante, esto no es necesariamente una regla que explica su mayor riqueza de cultivos, ya que el agricultor podría ser un empresario de monocultivo orgánico, tal como sucede en otras regiones del mundo, o no estar temporalmente sujeto a una certificadora. La práctica de rotación difundida en estos agricultores, cuando la producción está certificada, se vincula con la exigencia de rotación programada de los cultivos establecida en las normativas orgánicas (IFOAM, 2007), lo cual contribuye a explicar el cultivo de varias especies. Sin embargo, en la zona de La Plata y alrededores, la mayor diversificación de las fincas orgánicas sugiere que puede estar influenciada, en parte, por el destino de sus productos. Al no existir un mercado propio mayorista orientado a los productos orgánicos, el agricultor debe orientarse hacia canales de comercialización de carácter más diverso (canales cortos de venta) lo que determinaría la necesidad de una mayor oferta de productos para satisfacer la demanda (Benencia et al., 2004; Stupino et al., 2008; Souza Casadinho et al., 2009). En este sentido, los agricultores utilizaron la venta directa al consumidor, a través de venta a domicilio o feria, minorista a través de supermercados, restaurantes o intermediarios. La venta directa mediante un reparto a domicilio, se reconoce como la forma más difundida entre los agricultores orgánicos bonaerenses. En esta modalidad el agricultor entrega en forma directa al consumidor una caja con 7 a 10 productos de hortalizas (Souza Casadhino et al., 2009). Esto implica que debe sostener una variedad de hortalizas acorde a los consumidores realizando una adecuada planificación de la siembra a fin de satisfacer la demanda. Más aún, como surge de las encuestas, algunos agricultores orgánicos con mayor nivel de organización suelen canalizar, adicionalmente, otros productos orgánicos (sean de origen animal o vegetal, primarios o elaborados) procedentes de otras regiones, que ellos no producen; estos productos son obtenidos a través de canjes mediante la intervención de organizaciones intermedias. Este mecanismo destaca la capacidad de respuesta del agricultor y la influencia de la demanda procedente de sus canales de comercialización.

Diversidad Vegetal Espontánea en Agroecosistemas Hortícolas de La Plata y su relación con diferentes estilos de Agricultura: Importancia 
Las fincas de bajos insumos constituyeron un subgrupo heterogéneo y variado donde las fincas fueron cercanas a las orgánicas en una mayor diversidad de cultivos o, a las convencionales intensivas por el uso selectivo de algún insumo (fertilizantes o herbicidas) o por la implementación de técnicas tradicionales como el riego por surco o el desmalezado físico. La situación socio-económica y las vías de comercialización del agricultor influirían en el manejo, acercándolo a uno u otro tipo principal. Este grupo de agricultores coincide con la clasificación de Benencia et al. (1994) en agricultores familiares medios, que buscan estrategias de resistencia para mantenerse en el mercado dado que las superficies son menores y no están capitalizados. Por lo tanto, deben volcarse a los cultivos de tipo intensivo en el uso de la tierra pero también en mano de obra. Asimismo, es más bajo el número de agricultores que acceden al invernáculo (Benencia et al., 2009b). De acuerdo a la situación económica y al precio de los productos en el mercado, realizan un uso selectivo de los insumos optando por el uso de herbicidas o el desmalezado físico o por abonos químicos u orgánicos (más costosos) disminuyendo a veces la frecuencia de aplicación (Benencia et al; 1992, Benencia et al., 2009b). En relación con esto, estos agricultores, se destacaron por un menor uso de fertilizantes nitrogenados de distinto origen y la opción principal del manejo mecánico o manual de las espontáneas en desmedro del uso de herbicidas (lo se observó en el menor espectro de productos utilizados y la baja frecuencia de uso en el campo y, la ausencia de esterilizadores en invernáculo). Además, los agricultores manifestaron poseer sólo uno o dos cultivos de mayor valor comercial en el que invierten más tiempo e insumos en su cuidado y la utilización esporádica de abonos. En cuanto a la comercialización, van cambiando sus alternativas comerciales y además de llevar su producción a centros regionales, incorporan la venta a diversos comercios minoristas como supermercados y verdulerías (Benencia et al., 2009c).

La fertilización orgánica fue común a todos los manejos, lo cual se explica a que, en la horticultura, es utilizada como abono de base para la mejora de la estructura del suelo. La relación opuesta entre la fertilización orgánica y el desmalezado físico podría deberse a que los métodos mecánicos pueden ser elegidos a contraposición de las prácticas más costosas como el uso de fertilizantes y herbicidas, tal como ocurre con los agricultores de bajos insumos. En los orgánicos no debe descartarse cierto efecto de un mayor umbral de tolerancia a otras especies, que implica una menor intervención con fines de control; en tanto en los agricultores de bajos insumos, razones económicas

Diversidad Vegetal Espontánea en Agroecosistemas Hortícolas de La Plata y su relación con diferentes estilos de Agricultura: Importancia 
suelen ser la causa de una reducción del control de malezas sea esto mediante la aplicación química o laboreo humano.

Los elementos de juicio principales que los horticultores de La Plata han tenido en cuenta para justificar la elección de los cultivos, independientemente del tipo de horticultura que practican, se relacionaron con aspectos comerciales como la mayor rentabilidad del cultivo seleccionado y las vías elegidas de venta de sus productos.

De lo señalado, se deduce que el gradiente representó un nivel de intensidad dado por el incremento en el uso de insumos y de herramientas y la disminución de la diversidad cultivada; en tanto que el cultivo de más especies se asoció con destinos de consumo más diversificados (Stupino et al., 2007, 2008). Aquellos agricultores que tendieron a un mercado mayorista, presentaron menos cultivos.

El gradiente de intensidad de manejo refleja la heterogeneidad del Cinturón Hortícola. Cada agricultor tiene diferente forma de "hacer y pensar", que los hace diferentes entre sí, pero su agroecosistema se asemeja a aquellos de similar contexto socioeconómico-productivo y a su lógica personal (cosmovisión) que permite reunirlos en grupos relativamente definidos de tipos de producción pero que en la realidad son segmentos arbitrarios de un gradiente de intensidad de manejo.

\subsection{CONCLUSIONES}

Los resultados presentados en este capítulo mostraron que el Cinturón Hortícola Platense presentó tres formas de manejo principales que se reúnen dentro de un gradiente de intensidad de manejo: convencional intensivo, convencional de bajos insumos u orgánico. La metodología utilizada permitió determinar que las diferencias encontradas entre estas categorías respondieron a un conjunto de variables de manejo además del uso o no de químicos.

A partir de los resultados se propone que las decisiones de manejo de cada agricultor determinan la particularidad de cada finca. Aquellas fincas cuyos agricultores presentan similar situación socioeconómica, forma de producir, y lógica personal, se reconocen como un grupo dentro de las categorías establecidas. Sin embargo, otros factores como el acceso a los insumos, y al mercado influencian en las decisiones del 
agricultor. Por lo tanto, el Cinturón Hortícola representaría un gradiente de intensidad de manejo dinámico.

La tendencia actual es hacia la simplificación de los agroecosistemas y la especialización relativa en pocos cultivos e intensivos en insumos, tal como se observó en los sistemas convencionales intensivos. Sin embargo, en el Cinturón Hortícola Platense se mantienen sistemas menos intensivos como los orgánicos y los de bajos insumos, más diversificados, que se diferencian del modelo predominante.

En el próximo capítulo se analiza en qué medida el manejo determina la diversidad de espontáneas a escala parcela de cultivo.

\subsection{BIBLIOGRAFÍA}

Alconada M, Cuellas M, Poncetta P, Barragán S, Inda E \& Mitidieri A. 2011. Fertirrigación en cultivo de tomate protegido: Nutrición nitrogenada. Efectos en el suelo y en la producción. Horticultura Argentina: 30 (72) Mayo-Agosto.

Altieri MA. 1999. The ecological role of biodiversity in agroecosystems. Agriculture, Ecosystems and Environment 74: 19-31.

Ander EE. 1985. Metodología del trabajo social. Ed. El Ateneo, México.

Archenti A, Ringuelet R \& Salva MC. 1993. Los procesos de diferenciación de los productores hortícolas de La Plata. Etnia, n³8/39.

Barsky A. 2005. El periurbano productivo, un espacio en constante transformación. Introducción al estado del debate, con referencias al caso de Buenos Aires. Scripta Nova cripta Nova, Revista Electrónica de Geografía y Ciencias Sociales 9(194): 36. Disponible en: http://www.ub.es/geocrit/sn/sn-194-36.htm.

Benencia R \& Souza Casadinho J. 1993. Alimentos y salud: uso y abuso de pesticidas en la horticultura bonaerense. Realidad Económica 114-115: 29-53.

Benencia R. 1994. La horticultura Bonaerense: lógicas productivas y cambios en el mercado de trabajo. Desarrollo Económico 34(133): 53-73.

Benencia R \& Souza Casadinho J. 2009a. Introducción. En: Benencia R, Quaranta Q \& Souza Casadinho J (coord.), Cinturón hortícola de la Ciudad de Buenos Aires. Cambios sociales y productivos. Ediciones Ciccus, Buenos Aires. Cap 1: 11-35.

Benencia R, Quaranta G, \& Tassara C. 2009b. Estructura social agraria, producción y tecnología en el cinturón hortícola de la ciudad de Buenos Aires. En: Benencia R, Quaranta Q \& Souza Casadinho J (coord.), Cinturón hortícola de la Ciudad de Buenos

Diversidad Vegetal Espontánea en Agroecosistemas Hortícolas de La Plata y su relación con diferentes estilos de Agricultura: Importancia 
Aires. Cambios sociales y productivos. Ediciones Ciccus, Buenos Aires. Cap 2: 3970.

Benencia R, Cattáneo CA \& Fernández R. 1992. Consecuencias de un proceso de adopción tecnológica reciente: cultivos hortícolas bajo invernáculo en el cinturón verde de Buenos Aires. Centro de Estudios e Investigaciones Laborales CEIL. Documento de Trabajo No. 31, Buenos Aires.

Benencia R \& Souza Casadinho J. 2009c. Estrategias de productores resistentes en la horticultura de Buenos Aires en Cinturón Hortícola de la Ciudad de Buenos Aires. En: Benencia R, Quaranta Q \& Souza Casadinho J (Coord.), Cinturón hortícola de la Ciudad de Buenos Aires. Cambios sociales y productivos. Ediciones Ciccus, Buenos Aires. Cap 3: 71-84.

Bengtsson J, Ahnstrom J \& Weibull AC .2005. The effects of organic agriculture on biodiversity and abundance: a meta-analysis. Journal of Applied Ecology 42: 261269.

Bocchicchio AM \& Cattáneo CA. 2009. Comercialización, regulaciones y mercados frutihortícolas en el área metropolitana de Buenos Aires. En: Benencia R, Quaranta Q \& Souza Casadinho J (Coord.), Cinturón hortícola de la Ciudad de Buenos Aires Cambios sociales y productivos. Ediciones Ciccus, Buenos Aires. Cap 7: 155-194.

Bocero SL. 2002. Cultivos protegidos y problemas ambientales: un estudio de la horticultura marplatense en la década del noventa. Tesis de Maestría, Facultad Latinoamericana de Ciencias Sociales, Universidad Nacional de Mar del Plata.

Casadinho JS, Druán P. \& Moya M. 2009. Estudio de la producción orgánica en el Área Hortícola bonaerense. En: Benencia R, Quaranta Q \& Souza Casadinho J (Coord.), Cinturón hortícola de la Ciudad de Buenos Aires: Cambios sociales y productivos. Ediciones Ciccus, Buenos Aires. Cap 11: 275-292.

CASAFE. 2002. Guía de Productos Fitosanitarios para la Argentina. Cámara de sanidad agropecuaria y fertilizantes de la República Argentina, Buenos Aires, Argentina.

Cieza R. 2004. Asesoramiento Profesional y manejo de nuevas tecnologías en unidades de producción hortícolas del Gran La Plata, Argentina. Scientia Agraria 5(1-2): 79-85.

Fields PG \& White NDG. 2002. Alternatives to methyl bromide treatments for storedproduct and quarantine insects. Annual Review of Entomology 47: 331-359

De la Fuente EB \& Suárez S. 2008. Problemas ambientales asociados a la actividad humana: la agricultura. Ecología Austral 18: 239-252.

Digby PGN \& Kempton RA.1987. Multivariate analysis of ecological communities. Chapman \& Hall. $203 p$

Del Pino A, Repetto C, Mori C \& Perdomo C. 2008. Patrones de descomposición de estiércoles en el suelo. Terra Latinoamericana 26: 43-52.

García M \& Hang G. 2007. Impacto de la devaluación de principios de 2002 en el Cinturón Hortícola Platense. Estrategias tecnológicas adoptadas, sus resultados y

Diversidad Vegetal Espontánea en Agroecosistemas Hortícolas de La Plata y su relación con diferentes estilos de Agricultura: Importancia 
consecuencias. Centro de Estudios Histórico Rurales, Universidad Nacional de La Plata, Mundo Agrario, $8 \mathrm{n}^{\circ} 15$.

García M. 2010. Inicios, consolidación y diferenciación de la horticultura platense. En Svetliza de Nemirovky A (Coord), Globalización y agricultura periurbana en la Argentina. Escenarios, recorridos y problemas. Maestría en Estudios Sociales Agrarios, FLACSO, Buenos Aires, Serie Monografías 1.

Hang G, Kebat C, Bravo M, Larrañaga G, Seibane C, Ferraris G, Otaño M \& Blanco V. 2010. Identificación de Sistemas de Producción Hortícola en el Partido de La Plata, Provincia de Bs. As. Revista Bioagro 22(1): 81-86.

Hole DG, AJ Perkins, JD Wilson, IH Alexander, PV Grice \& Evans AD. 2005. Does organic farming benefit biodiversity? Biological Conservation 122:113-130.

IFOAM. 2007. Normas de IFOAM para la produccción y el procesamiento orgánicos. Disponible en https://www.ifoam.bio/en/our-library.

Kuepper G. 2003. Manures for organic crop production. Fundamentals of Sustainable Agriculture. Appropriate Technology Transfer for Rural Areas (ATTRA), USA. Disponible en http://attra.ncat.org/attra-pub/PDF/manures.pdf.

Matson PA, Parton WJ, Power AG \& Swift MJ.1997. Agricultural intensification and Ecosystems properties. Science 277: 504-509.

MBTOC (Methyl Bromide Technical Options Committee), 1994. Report of the methyl bromide technical options committee. Montreal protocol on substances that deplete the ozone layer. UNEP, Kenya: 304 p.

Oliveros Y, de Vasilio B \& Farfan C. 2012. Efecto del nivel de amonio ambiental sobre el comportamiento de los pollos de engorde. Revista Producción y negocio. Artículo técnico Venezuela: Disponible en: http://www.produccionynegocio.com/edicion_27/Amonio.htm.

Pascuali J. 1980. en: "El reciclaje de materias orgánicas en la agricultura de América". Boletín de Suelos de la FAO 51: 161-167.

Salazar Sosa E, Fortiz Hernández M, Vásquez Alarcón A, \& Vásquez Vásquez C. 2003. Abonos orgánicos y plasticultura. Gómez Palacio, México, Facultad de Agricultura y Zootecnia de la UJED, Sociedad Mexicana de la Ciencia del Suelo, COC y TED 2003. 233 pp. Disponible en http://www.smcs.org.mx/pdf/libros/abonos_org.pdf

Sarandón SJ. 2002. El agroecosistema: un sistema natural modificado. Similitudes y diferencias entre ecosistemas naturales y agroecosistemas. En: Sarandón SJ (Ed.). Agroecología. El camino hacia una agricultura sustentable. Ediciones Científicas Americanas, La Plata. 6: 119-134.

Sarandón SJ. 2009. Biodiversidad, agrobiodiversidad y agricultura sustentable: Análisis del Convenio sobre Diversidad Biológica. En: Tomas Leon Siccard, Miguel A. Altieri (Eds.). Vertientes del pensamiento agroecológico: fundamentos y aplicaciones., IDEAS 21, Sociedad Científica Latinoamericana de Agroecología (SOCLA), Universidad Nacional de Colombia, Opciones Graficas Editores, Bogotá, Colombia, 4: 105-130.

Diversidad Vegetal Espontánea en Agroecosistemas Hortícolas de La Plata y su relación con diferentes estilos de Agricultura: Importancia 
Schmitzberger I, Wrbka T, Steurer B, Aschenbrenner, G, Peterseil J \& Zechmeister H. 2005. How farming styles influence biodiversity maintenance in Austrian agricultural landscapes. Agriculture, Ecosystems and Environment 108: 274-290.

Stern VM, Smith RE, van Den Bosch R \& Kenneth SH. 1959. The integrated control concept. Hilgardia 20 (2):81.

Souza Casadinho J \& Bocero S. 2008. Agrotóxicos: Condiciones de utilización en la horticultura de la Provincia de Buenos Aires (Argentina), Revista Iberoamericana de Economía Ecológica 9: 87-101.

Souza Casadinho J. 2009. "Estudio de la producción orgánica en el Área hortícola Bonaerense". En: Benencia R, Quaranta Q \& Souza Casadinho J (Coord.), Cinturón hortícola de la Ciudad de Buenos Aires. Cambios sociales y productivos. Ediciones Ciccus, Buenos Aires. 32-45.

Stupino SA, Ferreira AC, Frangi JL \& Sarandón SJ. 2007. Agrobiodiversidad vegetal en sistemas hortícolas orgánicos y convencionales (La Plata, Buenos Aires, Argentina). Revista Brasileira de Agroecologia, Asociación Brasilera de Agroecología, Edición especial: Resúmenes del II Congreso Brasileiro de Agroecología. Noviembre de 2004. 2(1): 339-342.

Stupino SA, Frangi JL, Sarandón SJ, Arturi MF \& Ferreira AC. 2008. Plant diversity in two farms under organic and conventional management in La Plata, Argentina. A case study. Revista Brasilera de Agroecología 3(3): 24-35.

Swift MJ, MN Izac \& van Noordwijk M. 2004. Biodiversity and ecosystem services in agricultural landscapes-are we asking the right questions?. Agriculture, Ecosystems and Environment 104: 113-134.

Tang L, Wan K, Chuanpeng C \& Chen F. 2013. Effect of fertilization patterns on the assemblage of weed communities in an upland winter wheat field. Journal of Plant Ecology 7(1): 39-50.

Tapia Contreras F, Osorio Ulloa F. 1999. Conceptos sobre diseño y manejo de riego presurizado, Cartilla técnica. INIA, Intihuasi. $12 \mathrm{p}$.

Van der Ploeg JD 1993. Rural sociology and the new agrarian question. Sociologia Ruralis 33(2): 240-260.

Diversidad Vegetal Espontánea en Agroecosistemas Hortícolas de La Plata y su relación con diferentes estilos de Agricultura: Importancia 


\section{CAPÍTULO 5}

ANÁLISIS DE LA DIVERSIDAD DE PLANTAS ESPONTÁNEAS EN PARCELAS CULTIVADAS BAJO DIFERENTE MANEJO. ESCALA ALFA 


\title{
CAPÍTULO 5
}

\author{
Mira profundamente dentro de la naturaleza $y$ \\ entonces comprenderás todo mejor
}

Albert Einstein (1879-1975). Físico

\section{ANÁLISIS DE LA DIVERSIDAD DE PLANTAS ESPONTÁNEAS EN PARCELAS CULTIVADAS BAJO DIFERENTE MANEJO. ESCALA ALFA}

\subsection{INTRODUCCIÓN}

Una de las consecuencias del proceso de intensificación de la agricultura, ha sido la disminución en la diversidad de especies de plantas que crecen de manera espontánea junto a los cultivos (Sutcliffe \& Kay, 2000; van Elsen, 2000; Benton et al., 2003). Estas especies tienen un valor de conservación y, además, proveen de una variedad de servicios ecológicos a los agroecosistemas (Altieri, 1999; Benton et al., 2003, Marshall et al. 2003; Swift et al., 2004; Bárberi et al., 2010). Desde la mirada de la agricultura sustentable se propone diseñar sistemas de producción que contengan y se beneficien de la biodiversidad, para potencian los procesos naturales que permiten disminuir el uso de insumos externos (Fisher et al., 2014).

Los factores que determinan la diversidad actúan sobre el conjunto regional de especies, a modo de filtros ecológicos organizados jerárquicamente en función de la escala espacial (Diamond, 1975; Terradas, 2001). En una escala local, donde pueden situarse los sistemas modificados, los factores antrópicos son esenciales en la determinación de las comunidades de plantas espontáneas (Andersson \& Milberg, 1998; Matthew et al., 2010). Las técnicas de manejo utilizadas mantienen a los agroecosistemas con características semejantes a etapas iniciales de la sucesión ecológica. Las prácticas agrícolas aumentan la disponibilidad de recursos, aseguran el establecimiento del cultivo, y eliminan o previenen la incidencia de las poblaciones que reducen los rendimientos (Poggio, 2012). En este proceso, los agricultores intervienen con sus decisiones de manejo en la estructura de las comunidades bióticas que acompañan a los cultivos. Sin embargo, el manejo no es uniforme, con lo cual la diversidad puede variar de acuerdo con su intensidad. La intensidad de manejo es entendida como un conjunto de prácticas que describen la forma de utilización de los

Diversidad Vegetal Espontánea en Agroecosistemas Hortícolas de La Plata y su relación con diferentes estilos de Agricultura: Importancia 
recursos e insumos y que expresan el grado de simplificación o "artificialización" del agroecosistema (Capítulo 4). Resulta el factor más importante en determinar la riqueza y la composición florística de espontáneas (Fried et al. 2008; Pal et al., 2013). En este sentido, los diferentes estilos de agricultura pueden tener distinta influencia sobre la diversidad ya que difieren en el tipo e intensidad de los filtros que determinan el ensamblaje de comunidades (Mattehw et al., 2010). Por lo tanto, la diversidad, la composición florística y funcional, pueden asociarse a diferentes grados de disturbio que son generados por las prácticas de manejo (McLaughlin \& Mineau, 1995; Collins \& Qualset, 1999; de la Fuente et al., 1999; Ghorbanali et al., 2011).

Se ha encontrado que los sistemas bajo manejo orgánico tienden a tener una mayor riqueza y diversidad de especies que los convencionales (Krebs et al., 1999; van Elsen, 2000; Hyvonen et al., 2003; Roschewitz et al. 2005; Gabriel et al., 2010; Edesi et al., 2012; Henckel et al., 2015). En estos sistemas se destaca además, la presencia de especies de ciclo de vida largo (bienales, perennes), nativas y raras (van Elsen, 2000; Stupino et al., 2008; Travlos et al., 2018). Sin embargo, no se conoce bien cuáles son las variables de manejo que permiten el mantenimiento de la biodiversidad vegetal (Gabriel et al., 2010). Una de las explicaciones por la que se atribuyen una mayor riqueza y diversidad de especies a los sistemas orgánicos es el uso reducido de insumos y una mayor tolerancia a la presencia de vegetación espontánea (Hole, et al. 2005). Algunos autores establecen que la disponibilidad limitada y el menor uso de nitrógeno, la aplicación de control mecánico, las rotaciones de cultivos y una diversidad de cultivos más elevada, originan condiciones más favorables para muchas especies de plantas silvestres (Laird \& Schamp, 2006; Kleijn et al.,2009; Matthew et al., 2010; Travlos et al., 2018).

Estudios previos encontraron que las plantas son el grupo de organismos que obtiene mayores beneficios del manejo orgánico, probablemente por la restricción del uso de herbicidas (Roschewitz et al. 2005; Rundlöf et al., 2010; Tuck et al., 2014). El uso de herbicidas produce cambios en la composición de especies espontáneas disminuyendo la diversidad (Ghersa \& Martínez-Ghersa, 2000; Marshall et al., 2002). Por ejemplo, reduce la abundancia de especies de hoja ancha susceptibles y favorece la presencia de pastos (Hyvonen \& Salonen, 2002; Shepherd et al., 2003). La declinación de especies, en general, se vincula a que muchas son sensibles a un amplio rango de herbicidas (Mc Laughlin \& Mineau, 1995; Ghersa \& Martínez- Ghersa, 2000). 
Existen varias escalas espaciales en las que se puede analizar la diversidad y, teniendo en cuenta sus componentes de riqueza y equitabilidad (como variable opuesta a la dominancia). Una de las escalas es la denominada diversidad Alfa, que representa la diversidad dentro de un hábitat o muestra (MacArthur, 1965; Whitaker 1977; Magurran, 1988; Terradas, 2001). En este capítulo se considera la parcela de cultivo como unidad de análisis de la diversidad Alfa.

Los estudios sobre agricultura orgánica se han realizado principalmente en regiones de Europa y Norte América y existen datos limitados en otras áreas del mundo (Bátary et al., 2011; Tuck et al., 2014). En nuestro país, la diversidad de la vegetación asociada a los cultivos se ha evaluado en sistemas extensivos (de la Fuente et al., 1999; Suárez et al., 2001; Poggio et al., 2005, Roschewitz et al., 2005). Sin embargo, existen pocos antecedentes en los que se haya analizado el efecto del manejo sobre la diversidad y composición de plantas espontáneas en fincas hortícolas (Stupino et al., 2008; Tuck et al., 2014), y en particular, comparando cultivos similares bajo diferente manejo.

Hay procesos ecológicos que sólo ocurren en el nivel de parcela de cultivo, como por ejemplo la competencia y la captación de recursos. Por lo tanto, determinar el efecto del manejo sobre la diversidad de espontáneas en los cultivos hortícolas (nivel Alfa), contribuye a establecer qué prácticas promueven el mantenimiento de la biodiversidad de interés agronómico en las fincas.

Los resultados presentados en el Capítulo 4 mostraron que en el Cinturón Hortícola de La Plata existen tres formas de manejo principales que se reúnen dentro de un gradiente de intensidad de manejo: orgánico, convencional de bajos insumos y convencional intensivo. Las diferencias encontradas entre estas categorías respondieron a un conjunto de variables de manejo. Los sistemas convencionales intensivos representaron la condición de mayor intensificación y especialización del gradiente. Esto se debió al cultivo de pocas especies repetidas en el tiempo con incorporación de grandes cantidades de insumos químicos, principalmente herbicidas y fertilizantes. Por el contrario, los sistemas orgánicos, menos intensivos, se caracterizaron por la ausencia de productos de síntesis química. Ambos sistemas se diferenciaron, además, en el nivel de espontáneas tolerado por el agricultor (umbral de malezas), siendo más alto en los orgánicos, lo que implica una menor intervención con 
fines de control. El umbral de malezas y el desmalezado químico fueron las variables de manejo de mayor magnitud que explicaron el gradiente en forma significativa.

Teniendo en cuenta que el manejo hortícola convencional se caracteriza, principalmente, por el uso de herbicidas y la baja tolerancia a la presencia de espontáneas, se puede hipotetizar que un menor número de especies será capaz de establecerse en los cultivos convencionales. Por el contrario, en sistemas menos perturbados se pueden desarrollar comunidades de espontáneas más diversas (Laird \& Schamp, 2006). Dado los antecedentes, es esperable que la diversidad y composición florística de las espontáneas sea menor hacia el extremo del gradiente donde aumenta la intensidad de las prácticas de manejo, es decir hacia los sistemas convencionales intensivos. Las variables que mejor explican esta relación son el uso de herbicidas y el nivel de malezas tolerado por el agricultor (umbral).

El objetivo de este capítulo es identificar, del conjunto de variables que definen las formas de manejo hortícola platense, aquellas que influyen de manera más relevante sobre la diversidad de plantas a escala parcela de cultivo (Alfa). Para ello, se ponen a prueba las siguientes hipótesis:

Hipótesis 1:

Los componentes de la diversidad de las parcelas de tres cultivos (tomate, lechuga y maíz), conducidos a campo, dan resultados distintos de acuerdo con la diferencia en la intensidad del manejo (orgánico y convencional intensivo).

- Los cultivos orgánicos debido a su manejo menos intensivo, presentan una mayor riqueza, equitabilidad y diversidad que el que expresan los mismos cultivos bajo manejo convencional intensivo.

Hipótesis 2:

Las diferentes intensidades de manejo afectan de distinta manera la composición florística de especies acompañantes de los cultivos.

- Las parcelas con similar manejo tienen mayor semejanza florística entre sí que las parcelas de diferente manejo. 
- Las parcelas convencionales son más parecidas entre sí en la composición de especies que las parcelas orgánicas.

Hipótesis 3:

La instalación de cultivos de ciclo corto implica conducir agroecosistemas que tienen características semejantes a aquellas de las etapas tempranas de la sucesión. La intensidad y duración de las técnicas aplicadas para causar o remover tensiones (stress) en cada tipo de manejo, afectan la composición estructural de grupos funcionales de plantas espontáneas.

Hipótesis 4:

La diversidad de plantas acompañantes se vincula con un complejo de variables, entre las que se hallan algunas asociadas con cada tipo de manejo, la historia de uso de la tierra, y la tolerancia del agricultor hacia las "malezas".

- La riqueza y diversidad de especies espontáneas disminuirán, principalmente con el aumento de uso de herbicidas y el menor umbral de daño considerado por el agricultor.

\subsection{METODOLOGÍA}

\subsubsection{Selección de las fincas}

La metodología de selección y localización de los establecimientos de producción hortícola fue descripta en el Capítulo 3.

\subsubsection{Muestreo de la vegetación espontánea}

El muestro de la vegetación se realizó en las parcelas cultivadas, utilizando un área de $25 \mathrm{~m}^{2}$ establecida a partir de una línea transecta de $25 \mathrm{~m}$ ubicada a lo largo de la parcela cultivada y contemplando $0,5 \mathrm{~m}$ a cada lado. La unidad de muestreo se ubicó en al menos una parcela de los distintos cultivos presentes en la finca, incrementándose el número de unidades en función de la superficie total de cada cultivo, a razón de una unidad muestral por hectárea. Los criterios de selección de la unidad de muestro, el 
método utilizado y la determinación de ejemplares colectados fueron explicados en mayor detalle en el Capítulo 3.

En cada unidad de muestreo, se registraron las especies presentes y se utilizó una escala combinada de cobertura-abundancia de Braun-Blanquet (Matteucci \& Colma, 1982) para estimar la importancia de las especies, necesaria para el cálculo posterior de índices de diversidad específica. Los valores de la escala son:

r: uno o pocos individuos.

+: menos del $5 \%$ de cobertura y ocasional.

1: abundantes pero con cobertura muy baja, o menos abundantes pero con mayor cobertura pero siempre menor al $5 \%$.

2: muy abundante y menos del $5 \%$ de cobertura; o menos abundante y de 5 a $25 \%$ de cobertura.

3: 25 a $50 \%$ de cobertura, independientemente del número de individuos.

4: 50 a $75 \%$ de cobertura, independientemente del número de individuos.

3: 75 a $100 \%$ de cobertura, independientemente del número de individuos.

\subsubsection{Análisis de la diversidad florística}

Para calcular la diversidad Alfa se consideraron los cultivos de mayor superficie total implantada en el Cinturón Hortícola de la Plata (CHFBA, 2006) y que fueron comunes a la mayoría de las fincas relevadas. Con este criterio se seleccionaron los cultivos de, tomate, lechuga y maíz realizados bajo manejo a campo (aire libre) e invernáculo (bajo cubierta).

Se utilizó como índice de diversidad Alfa, la riqueza de especies a partir de la lista total de especies presente en las unidades de muestreo de cada cultivo. En el caso de los cultivos que presentaron repeticiones en una misma finca (es decir, la especie cultivada se censó en más de una parcela), se seleccionó la unidad de muestreo (parcela) con mayor número de especies espontáneas para obtener un valor de riqueza de especies por cultivo (Alfa) en cada finca. Se calculó, además, el índice de diversidad de Shannon de estas parcelas para cada cultivo, $\mathrm{H}=-\Sigma \mathrm{pi}$ * $\mathrm{In} \mathrm{pi}$, donde pi es la abundancia proporcional de la especie i (Magurran, 1988). Para estimar la abundancia de las especies se convirtió el valor asignado de la escala combinada de Braun Blanquet en el valor porcentual medio del rango representado; para las clases $r$ y + se usaron los valores 0.5 y 1 respectivamente. Para el cálculo del índice de Shannon se utilizó el

Diversidad Vegetal Espontánea en Agroecosistemas Hortícolas de La Plata y su relación con diferentes estilos de Agricultura: Importancia 
programa MVSP 3.1, que indica además sus dos componentes: la riqueza y equitabilidad de las especies. En éste caso, especies por unidad de muestreo (parcela).

En cada cultivo, se calculó la riqueza, equitabilidad y el índice de diversidad de Shannon promedios entre los manejos más contrastantes, orgánico y convencional intensivo. Para ello, se utilizaron sólo los cultivos manejados a campo debido a que los cultivos de invernáculo eran menos representativos en el número de muestras.

\subsubsection{Análisis de la composición florística de los cultivos bajo diferente manejo}

Para establecer si existen diferencias en la composición florística entre parcelas de un mismo cultivo bajo distinto manejo, se analizó el grado de similitud en la composición de especies entre las muestras de los tres cultivos anteriormente seleccionados. Para ello, se realizó un análisis de conglomerados (Cluster), que consiste en agrupar un conjunto de observaciones en grupos o Cluster basado en el criterio de distancia o similitud. Para ello se utilizó el método de Varianza Mínima o método de Ward, utilizando el índice de Distancia Euclideana, en el programa Statistica 7.0. Cuando mayor es el valor del este índice, mayor es la distancia entre dos muestras o su disimilitud.

Para obtener información más detallada acerca de la composición florística y la distribución de especies en los cultivos de diferente manejo se aplicó el método Twispan (análisis de especies indicadoras de doble vía) (Hill, 1979) con el programa PCORD 6.0. Es un método de clasificación jerárquico, politético y divisivo, que divide comunidades y sitios simultáneamente en grupos sobre la base de la información de todas las especies. Se basa en la identificación de especies denominadas "diferenciales", que son aquellas que presentan preferencias ecológicas y su presencia está vinculada a diferentes grupos de censos. El resultado del método se aproxima a la tabla fitosociológica de BraunBlanquet (Matteucci \& Colma, 1982). Es una tabla de doble entrada (censos y especies) donde se pueden distinguir unidades de vegetación e inferir relaciones entre las comunidades y los factores ambientales (Roig, 1973). En este caso, mediante este análisis se trata de establecer relaciones entre los grupos de especies y su distribución en los cultivos bajo distinto manejo.

\subsubsection{Análisis de la estructura de las tres categorías consideradas bajo el concepto de grupos funcionales}

Diversidad Vegetal Espontánea en Agroecosistemas Hortícolas de La Plata y su relación con diferentes estilos de Agricultura: Importancia 
En cada cultivo bajo manejo a campo orgánico y convencional intensivo, se cuantificó la proporción de especies según, la morfología (monocotiledóneas, dicotiledóneas), el ciclo de vida (anuales, bienales y perennes) y el origen (exóticas y nativas). Se analizó, además, cómo se distribuyen las especies según el ciclo de vida en las plantas nativas y exóticas.

\subsubsection{Diversidad Alfa en función de las prácticas de manejo}

Se realizó una correlación entre las principales variables que definen el manejo hortícola platense, observadas en el Capitulo 4 (Tabla 3) y la riqueza y diversidad de especies espontáneas. Para ello, se seleccionaron aquellas variables significativas que podían tener influencia sobre la diversidad a escala parcela de cultivo: uso de esterilizadores del suelo, historia de uso, desmalezado químico, desmalezado físico, aplicación de fertilizantes químicos nitrogenados, ausencia de rotaciones, intensidad de uso del suelo, riego y umbral de malezas. Asimismo se exploraron otras variables que no fueron significativas como, el control físico de malezas y la fertilización orgánica. Se utilizó el coeficiente de correlación de Spearman con el programa Statistica 7.0, el cual resulta apropiado para analizar variables ordinales. El índice oscila entre -1 y +1 , indicando asociaciones negativas o positivas respectivamente. El valor numérico indica la magnitud de la correlación. Así, un valor cercano a 1 significa una buena correlación y cercano a 0 una correlación mínima o nula entre dos variables analizadas.

Para analizar la tendencia entre la riqueza y el gradiente de manejo obtenido en el Capítulo 4, se consideraron las parcelas con modalidades a campo e invernáculo juntas $(n=71)$. A su vez, se analizaron separadamente ambas modalidades, para tener más precisión en el grado de correlación entre la riqueza y las variables más características o exclusivas de un tipo de manejo (como el uso de esterilizadores que es propio del cultivo en invernáculo).

\subsection{RESULTADOS}

\subsubsection{Diversidad y composición florística entre cultivos bajo diferente manejo}

Los valores más altos de riqueza promedio estuvieron comprendidos entre 15 a 19 especies. El maíz orgánico tuvo el mayor número de especies (19 sp.). La lechuga bajo 
manejo convencional fue el cultivo que tuvo una riqueza significativamente menor ( $9 \mathrm{sp}$.) respecto del resto de los otros cultivos y manejos (Figura 5.1).

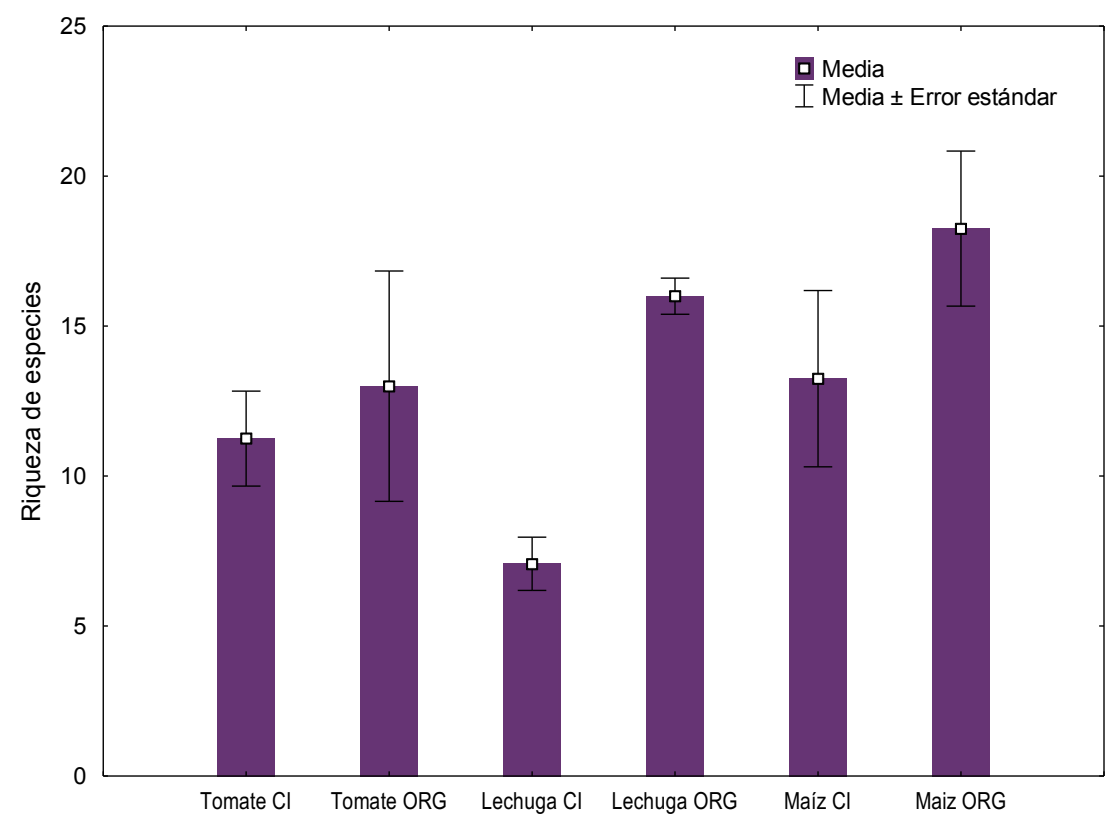

Figura 5.1. Riqueza promedio de especies en cultivos de tomate, lechuga y maíz, orgánicos (ORG) y convencionales intensivos $(\mathrm{Cl})$.

Los valores más altos de equitabilidad estuvieron comprendidos entre 0,75 y 0,81 (Figura 5.2). Al igual que con la riqueza, la lechuga convencional tuvo el menor valor de equitabilidad $(E=0,58)$, y mostró diferencias notorias entre manejos, siendo menos equitativo (más dominancia de especies) el cultivo convencional intensivo.

Los valores medios más altos del índice de Shannon estuvieron comprendidos entre 3 y 3,4. La lechuga convencional fue el cultivo que difirió en forma significativa de los demás especies bajo diferente manejo y tuvo la menor diversidad $(H=1,9)$. El maíz convencional y el tomate orgánico fueron los cultivos más variables en el índice respecto de los mismos cultivos bajo el manejo en comparación (Figura 5.3). 


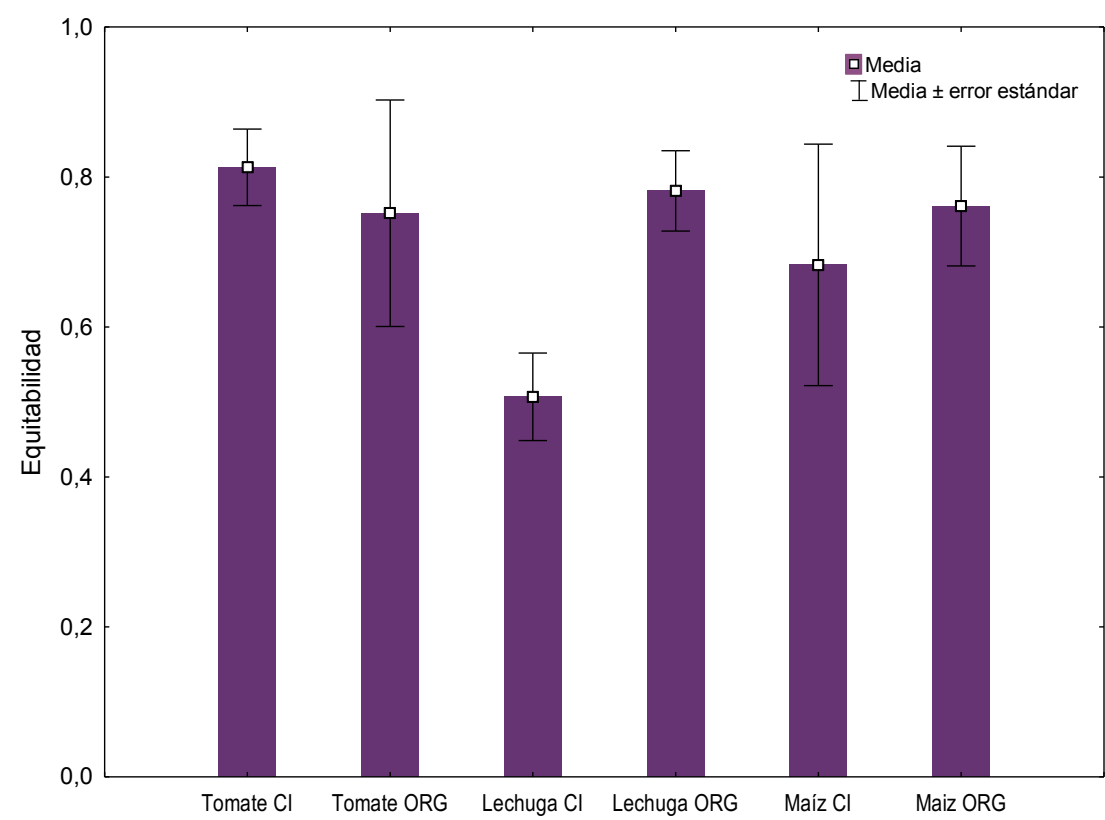

Figura 5.2. Valores de equitabilidad en cultivos de tomate, lechuga, y maíz, orgánicos (ORG) y convencionales intensivos $(\mathrm{Cl})$.

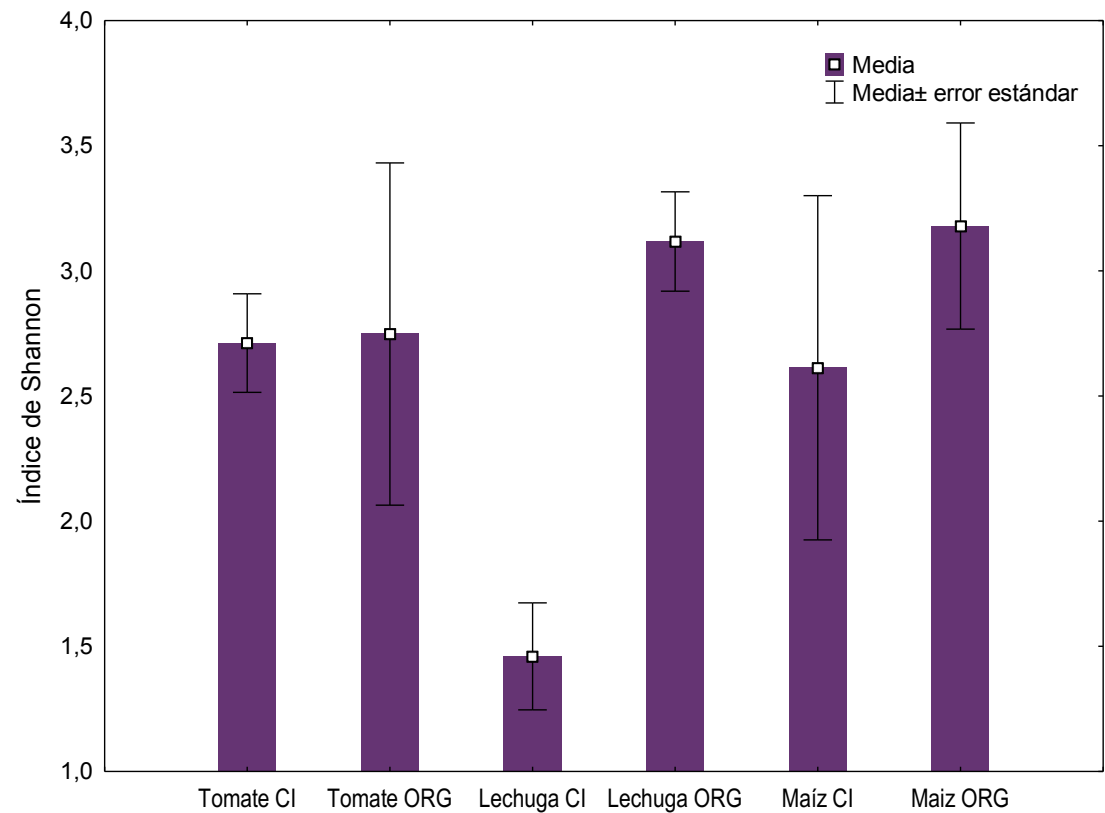

Figura 5.3. Índice de diversidad de Shannon en cultivos de tomate, lechuga, y maíz, orgánicos (ORG) y convencionales intensivos (Cl).

En general, se observo una tendencia a una mayor riqueza, equitabilidad y diversidad en los cultivos orgánicos, como lo muestran los valores medios de las barras de los gráficos. La lechuga, fue el cultivo que mostró diferencias significativas en las tres variables. Una misma tendencia se observa en maíz, con mayores valores de las 
variables mencionadas. El tomate, no presentó diferencias en ninguna de las variables, aunque la variabilidad fue mayor en el cultivo orgánico.

Cuando se analizaron los cladogramas, se observó que, en todos los cultivos, las muestras de similar manejo tendieron a agruparse entre sí. En el tomate, se conformaron 3 grupos en el nivel de corte de 6 (Figura 5.4 a). El grupo A reunió principalmente a O-Cl, el $\mathrm{B}$ a muestras $\mathrm{Cl}$, y el $\mathrm{C}$ a O-BI. Hubo una tendencia a reunirse muestras de similar manejo. No obstante, las muestras orgánicas se reunieron en grupos diferentes: las muestras T1 y T20 en el grupo A y las muestras T26, T27, T2 y T5 en el grupo $C$, estas últimas de cultivos bajo invernáculo. Las muestras orgánicas del grupo A se caracterizaron por una menor diversidad de especies respecto de $\mathrm{C}$, lo que las asemejó a los $\mathrm{Cl}$ de invernáculo.

En la lechuga, se diferenciaron dos ramas principales ( $A$ y $B$ ), con parcelas que reúnen a los orgánicos (A1) y los convencionales intensivos (A2, B1 y B2) (Figura 5.4 b). En el caso del maíz, las muestras se agruparon dentro de dos ramas principales del cladograma, A y B, a un nivel de corte de 4 (Figura 5.4 c). En el subgrupo A11, se reunieron las muestras orgánicas $(M 1, M 3)$ y en $A 12$, convencionales intensivos (M8, M9). El grupo B estuvo formado por parcelas de bajos insumos (B1) y, parcelas BI-CI (B2), que variaron en la composición.

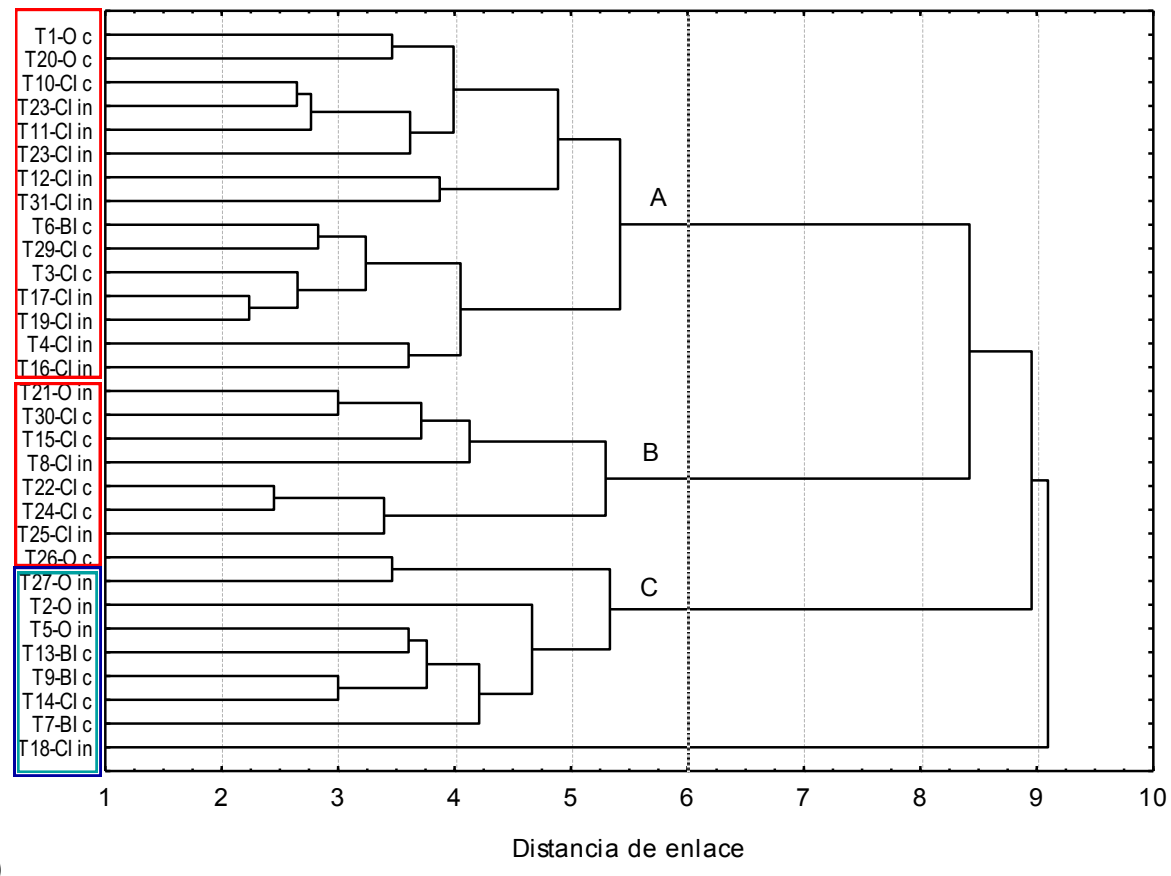

Diversidad Vegetal Espontánea en Agroecosistemas Hortícolas de La Plata y su relación con diferentes estilos de Agricultura: Importancia 


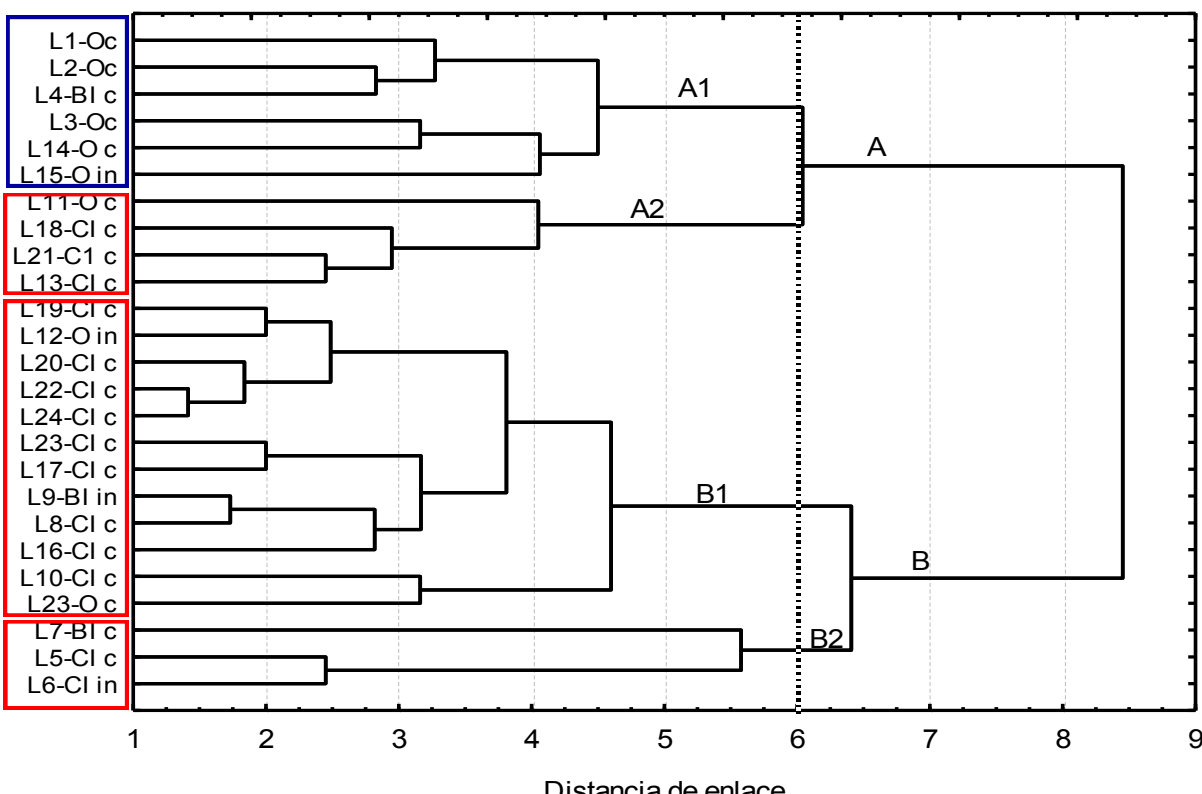

b)

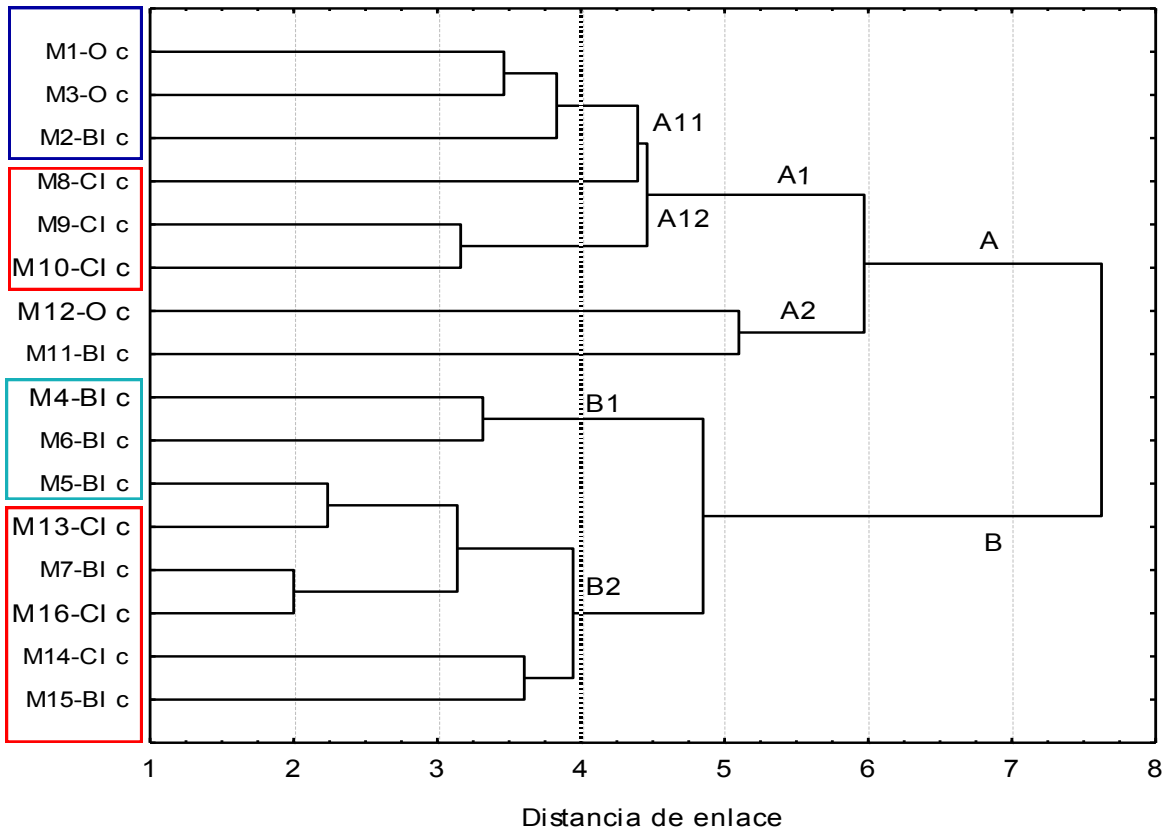

c)

Figura 5.4. Clasificación jerárquica de un conjunto de parcelas de cultivo mediante el método de conglomerados de Ward y la medida de distancia Euclideana. Menores distancias significan mayor semejanza en la composición florística entre las parcelas y/o los grupos conformados para los cultivos: a) tomate $(\mathrm{T})$, b) lechuga $(\mathrm{L})$, c) maíz $(\mathrm{M})$, bajo manejo orgánico $(\mathrm{O})$, convencional de bajos insumos $(\mathrm{BI})$ y convencional intensivo $(\mathrm{Cl})$, con modalidades a campo (c) e invernáculo (in). Los recuadros de color indican grupos de muestras que pertenecen, mayormente, a un tipo de manejo: rojo: convencional intensivo; azul: orgánico y celeste: bajos insumos. 
En general, en cada cultivo, las muestras convencionales de bajos insumos se ubicaron cercanas a muestras tanto orgánicas como convencionales intensivas.

Se encontró una similitud cualitativa en valores intermedios a altos en la composición florística, independientemente del cultivo y el tipo de manejo. Esto se observó en la distribución de los grupos conformados, cuyas distancias de enlace fueron en su mayoría menores a 5. En el caso del manejo convencional intensivo, la variabilidad dentro de los grupos de muestras fue mayor respecto de los otros manejos con un rango de amplitud mayor en el índice, donde hubo muestras que se parecieron más y otras muy poco en la composición de especies.

En el análisis de la composición y distribución de especies (Método de Twinspan), se observaron diferencias en los tipos de manejo. Hubo una tendencia a agruparse muestras de menor intensidad de manejo por un lado, e intensivas por otros, más que por el tipo de cultivo.

El maíz fue el cultivo que más especies exclusivas y de frecuencia intermedia presentó y especialmente en los sitios orgánicos. El tomate convencional presentó una mayor presencia de especies pero de baja frecuencia y abundancia (comprendidas entre el orden 77 a 97 de la tabla).

Algunas especies estuvieron sólo presentes en los manejos de menos intensidad, como Datura ferox y Salpichroa origanifolia y, menos representadas, Echium plantagineum, Potentilla vesca, y Solanum sisymbrifolium. Otras especies fueron generalistas con tendencia a una mayor frecuencia en los cultivos orgánicos y de bajos insumos (comprendidas entre el orden 24 a 32 de la tabla): Digitaria sanguinalis, Rumex crispus, Alternanthera philoxeroides, Chenopodium album, Anoda cristata, Echinochloa crus-galli, Lamium amplexicaule, Matricaria chamomilla y Veronica persica.

Algunas de las especies encontradas en los convencionales fueron mencionadas como resistentes a los herbicidas: Chenopodium album, Poa annua, Echinochloa colona, Echinochloa crus-galli, Lolium multiflorum, Amaranthus hybridus, Cyperus rotundus (SENASA, 2016). 
En general, los valores de abundancia de las especies fueron bajos, con mayor tendencia a una mayor cobertura-abundancia en los cultivos orgánicos. El tomate convencional fue el cultivo de menor frecuencia y abundancia.

Tabla 5.1. Tabla de especies (filas) versus muestras de cultivos (columnas) de tomate (T) Lechuga (L) y Maíz (M).Seguido a las abreviaturas se expresan los distintos manejos: orgánico $(\mathrm{O})$, bajos insumos $(\mathrm{BI})$ y convencional intensivo (C), respectivamente. Por ejemplo, TC representa tomate convencional intensivo. Los símbolos romanos de los datos representan la frecuencia de cada especie en cada muestra ( $\mathrm{a} V$, siendo $\mathrm{V}$ la mayor frecuencia), seguidos de valores de cobertura más frecuente según la escala cobertura abundancia de Braun-Blanquet.

\begin{tabular}{|c|c|c|c|c|c|c|c|c|c|c|}
\hline & $\mathrm{N}^{\circ}$ de especies & 65 & 32 & 31 & 36 & 37 & 45 & 27 & 39 & 25 \\
\hline & $N^{\circ}$ de censos (n) & 20 & 14 & 6 & 3 & 7 & 7 & 3 & 7 & 4 \\
\hline & Tipo de cultivo & TC & LC & MC & MO & LO & TO & LBI & MBI & TBI \\
\hline & \multicolumn{10}{|l|}{ Especies } \\
\hline 1 & Ammi majus & - & - & I.+ & II.r & - & - & - & - & - \\
\hline 2 & Trifolium pratense & - & - & - & II.+ & - & - & - & - & - \\
\hline 3 & Lythrum hyssopifolia & & & & II.+ & & & & & \\
\hline 4 & Bowlesia incana & - & - & - & II.+ & - & - & - & - & - \\
\hline 5 & Malva neglecta & - & - & I.r & - & - & - & - & - & - \\
\hline 6 & Bidens subalternans & - & - & - & II.+ & - & & - & - & - \\
\hline 7 & Echium plantagineum & - & - & - & II.1 & - & - & II.+ & - & - \\
\hline 8 & Datura ferox & - & - & - & II.r & II.r & II.+ & II. + & I.r & - \\
\hline 9 & Potentilla vesca & - & - & - & - & I.+ & l.+ & - & - & - \\
\hline 10 & Allium porrum & - & - & - & - & I.+ & - & - & - & - \\
\hline 11 & Setaria verticillata & - & - & - & - & - & II.+ & - & - & - \\
\hline 12 & Salpichroa origanifolia & - & - & - & - & - & I.r & II.+ & I.+ & - \\
\hline 13 & Ambrosia tenuifolia & - & - & - & 11.2 & - & I.+ & - & - & - \\
\hline 14 & Solanum sisymbriifolium & - & - & - & - & - & I.r & - & I.r & - \\
\hline 15 & Veronica peregrina & - & - & - & - & I.+ & - & - & - & - \\
\hline 16 & Cyperus surinamensis & - & - & - & - & - & I.r & - & - & - \\
\hline 17 & Anthemis cotula & - & - & - & - & - & - & II.+ & - & - \\
\hline 18 & Raphanus sativus & - & - & - & - & - & - & - & - & II.r \\
\hline 19 & Amaranthus viridis & - & - & - & - & - & I.r & - & - & - \\
\hline 20 & Pherthenium & - & - & - & - & - & - & - & I.+ & - \\
\hline 21 & Oxalis articulata & - & - & - & - & I.r & - & - & l.+ & - \\
\hline 22 & Capsicum annum & - & - & - & - & I.r & - & - & - & - \\
\hline 23 & Allium fistulosum & - & - & - & - & I.+ & - & - & - & - \\
\hline 24 & Digitaria sanguinalis & 1.2 & - & - & - & III.+ & III.1 & II.+ & l.+ & VI.+ \\
\hline 25 & Rumex crispus & I.+ & - & VI.+ & VI.1 & V.2 & 11.2 & VI.1 & Vl.+ & Vl.+ \\
\hline 26 & Alternanthera & I.+ & - & II.1 & II.1 & 1.1 & VI.1 & VI.1 & 111.2 & VI.2 \\
\hline 27 & Chenopodium album & I.+ & I.r & - & VI.+ & III.1 & III.+ & II.+ & VI.1 & II.+ \\
\hline 28 & Anoda cristata & - & I.r & - & VI.+ & II.+ & II.1 & II.+ & III.1 & II.+ \\
\hline 29 & Echinochloa crus-galli & 1.1 & III.+ & III.+ & V.1 & V.+ & V.1 & VI.1 & V.+ & V.1 \\
\hline 30 & Lamium amplexicaule & I.+ & 1.1 & VI.1 & VI.+ & II.1 & II.+ & $11 . .+$ & 1.1 & Vl.+ \\
\hline 31 & Matricaria chamomilla & I.+ & - & II.+ & VI.1 & III.+ & III.+ & II.+ & 1.1 & Il.r \\
\hline 32 & Veronica persica & I.+ & 1.1 & III.1 & V.+ & II.+ & II.1 & II.+ & II.1 & V.+ \\
\hline 33 & Jaborosa runcinata & I.r & - & - & - & 1.1 & - & - & 1.3 & II.1 \\
\hline 34 & Panicum bergii & I.+ & - & - & - & 1.1 & II.1 & - & - & II.r \\
\hline 35 & Cynodon dactylon & II.+ & I.+ & I.+ & - & III.+ & 111.2 & II. + & III.1 & - \\
\hline 36 & Taraxacum officinale & I.r & - & - & - & - & II.r & - & - & II.+ \\
\hline 37 & Anagallis arvensis & I.+ & - & - & - & I.+ & I.r & II.r & - & - \\
\hline 38 & Cyperus rotundus & 1.1 & - & - & - & - & - & II.+ & 11.2 & - \\
\hline
\end{tabular}


39 Carduus acanthoides

40 Lolium multiflorum

41 Vicia sativa

42 Circium vulgare

43 Polygonum persicaria

44 Amaranthus hybridus

45 Cichorium intybus

46 Convolvulus arvensis

47 Lepidium didymum

48 Echinochloa colona

49 Eragrostis mexicana

50 Galinsoga parviflora

51 Leptochloa chloridiformis

52 Lotus glaber

53 Portulaca oleracea

54 Sonchus oleraceus

55 Lactuca sativa

56 Polygonum aviculare

Cyclospermum

8 Stellaria media

9 Urtica urens

60 Dichondra microcalyx

Nicotiana longiflora

Oxalis corniculata

Stachys arvensis

4 Capsella bursa-pastori

Conium maculatum

6 Plantago lanceolata

7 Amaranthus lividus

8 Picris echioides

69 Sida rhombifolia

70 Trifolium repens

71 Cucurbita maxima

72 Conyza bonariensis

73 Gamochaeta platensis

74 Acicarfha tribuloides

75 Senecio vulgaris

76 Poa annua

77 Paspalum dilatatum

78 Physalis viscosa

79 Wedelia glauca

80 Euphorbia serpens

81 Solanum sarrachoides

82 Verbena litoralis litoralis

83 Verbena bonariensis

84 Setaria parviflora

85 Geranium molle

86 Phyla canescens

87 Morus Alba

88 Eruca vesicaria

89 Echinochloa crus-pavonis

90 Sida cordifolia

91 Lonicera japonica

92 Distichlis spicata

93 Acicarpha procumbens

94 Medicago polymorpha

95 Solanum chenopodioides

96 Geranium rotundifolium

97 Fraxinus excelsior

Diversidad Vegetal Espontánea en Agroecosistemas Hortícolas de La Plata y su relación con diferentes estilos de Agricultura: Importancia 


\subsubsection{Análisis de la composición de especies según los grupos funcionales}

Las especies exóticas, anuales y dicotiledóneas, fueron las más abundantes en ambos tipos de manejo, orgánico y convencional (Figura 5.5). Las exóticas predominaron en la lechuga y el maíz convencionales. Los cultivos orgánicos tuvieron un mayor número de especies nativas que los convencionales (a excepción del tomate). Además, tuvieron una mayor proporción de especies de ciclo de vida largo. En el maíz orgánico, las especies bienales tuvieron un valor más alto que en los cultivos convencionales. En la lechuga orgánica las especies perennes fueron más abundantes respecto de la otra modalidad de manejo. La proporción de dicotiledóneas superó a las monocotiledóneas en los distintos cultivos. Sin embargo, las diferencias entre manejos en cuanto al tipo morfológico fueron variables entre las distintas especies cultivadas. 

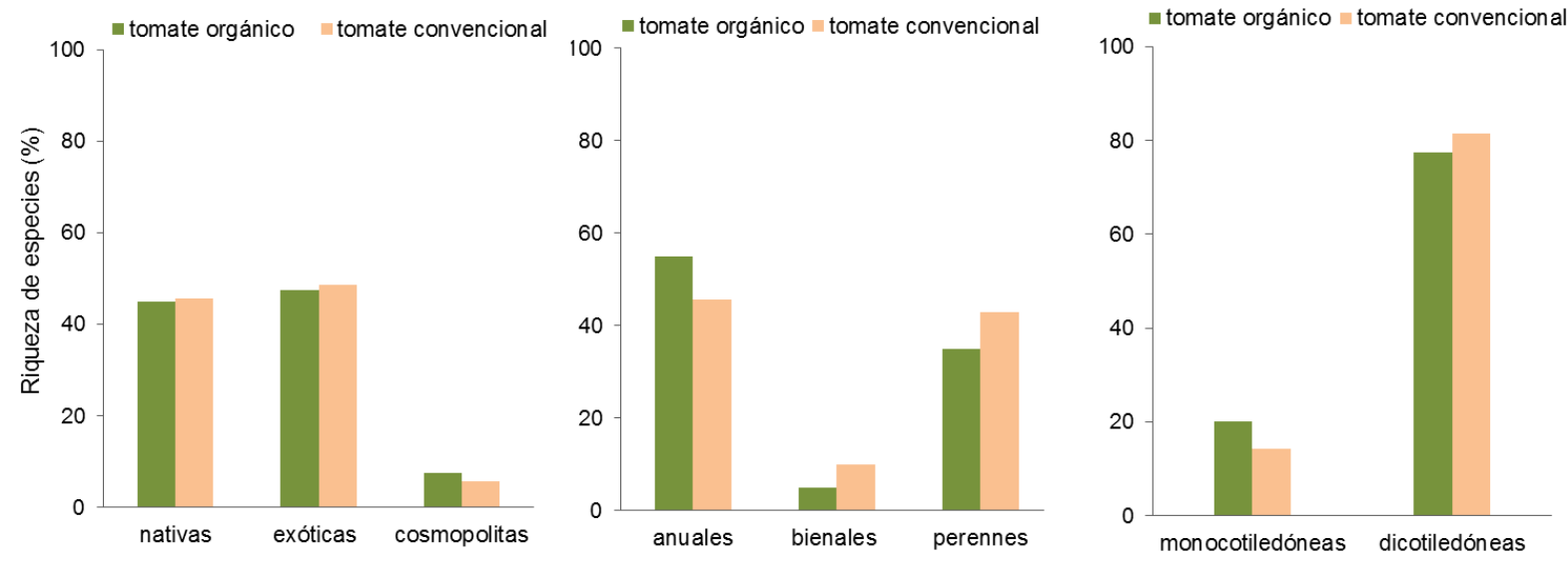

a)
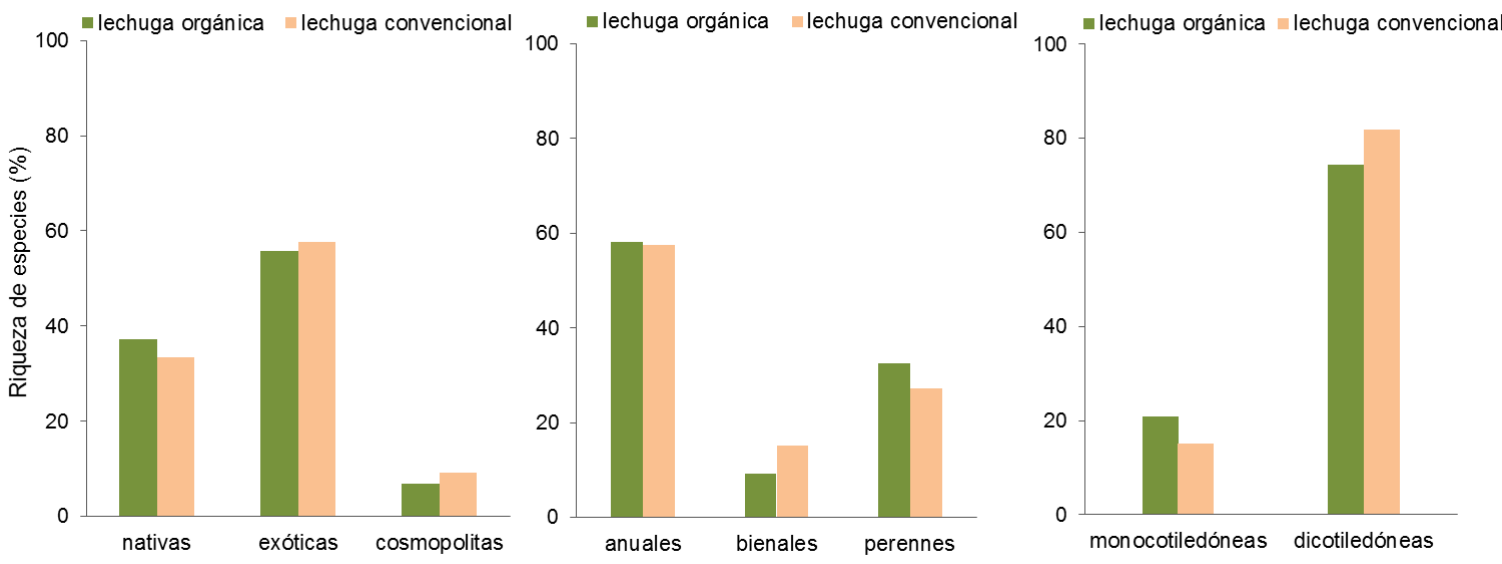

b)
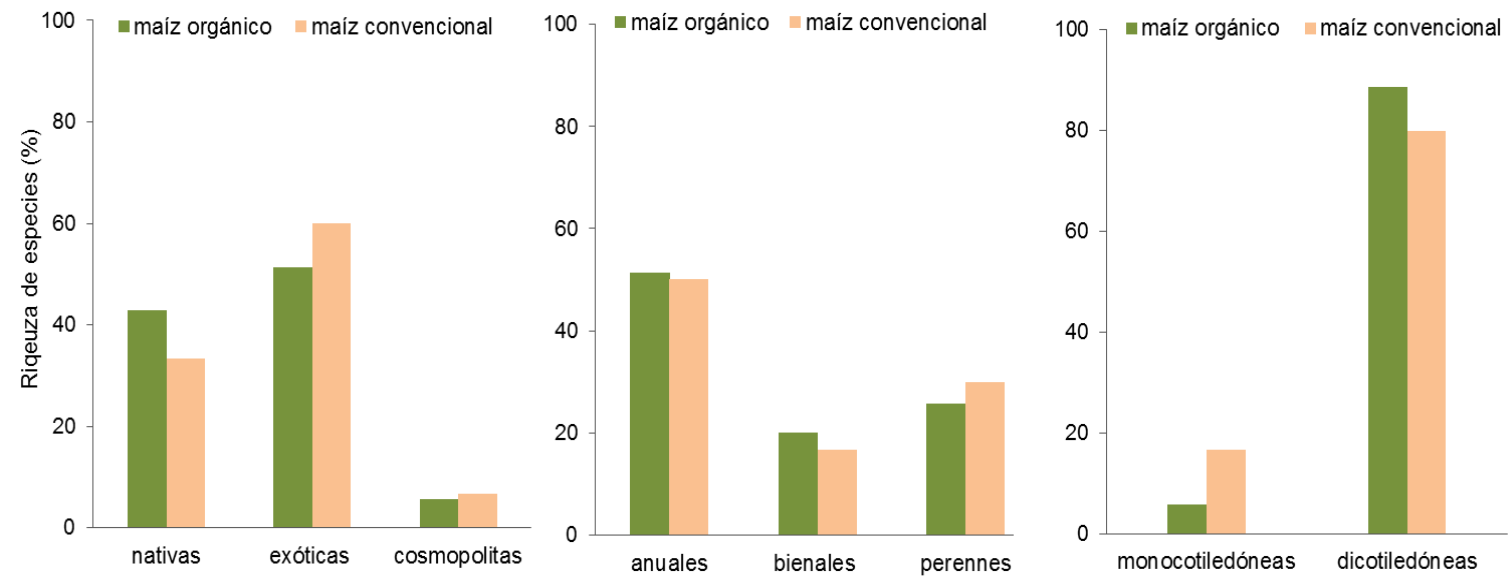

c)

Figura 5.5. Proporción de especies según el origen, ciclo de vida y morfología de plantas espontáneas en cultivos de tomate (a), lechuga (b) y maíz (c), orgánicos y convencionales intensivos.

Diversidad Vegetal Espontánea en Agroecosistemas Hortícolas de La Plata y su relación con diferentes estilos de Agricultura: Importancia para la sustentabilidad 
Las especies tanto exóticas como nativas fueron en su mayoría anuales en los tres cultivos bajo diferente manejo (tomate, lechuga y maíz) (Figura 5.6). A su vez, en el manejo orgánico las nativas perennes fueron más abundantes que en el convencional, alcanzando cerca del $50 \%$ en la lechuga.
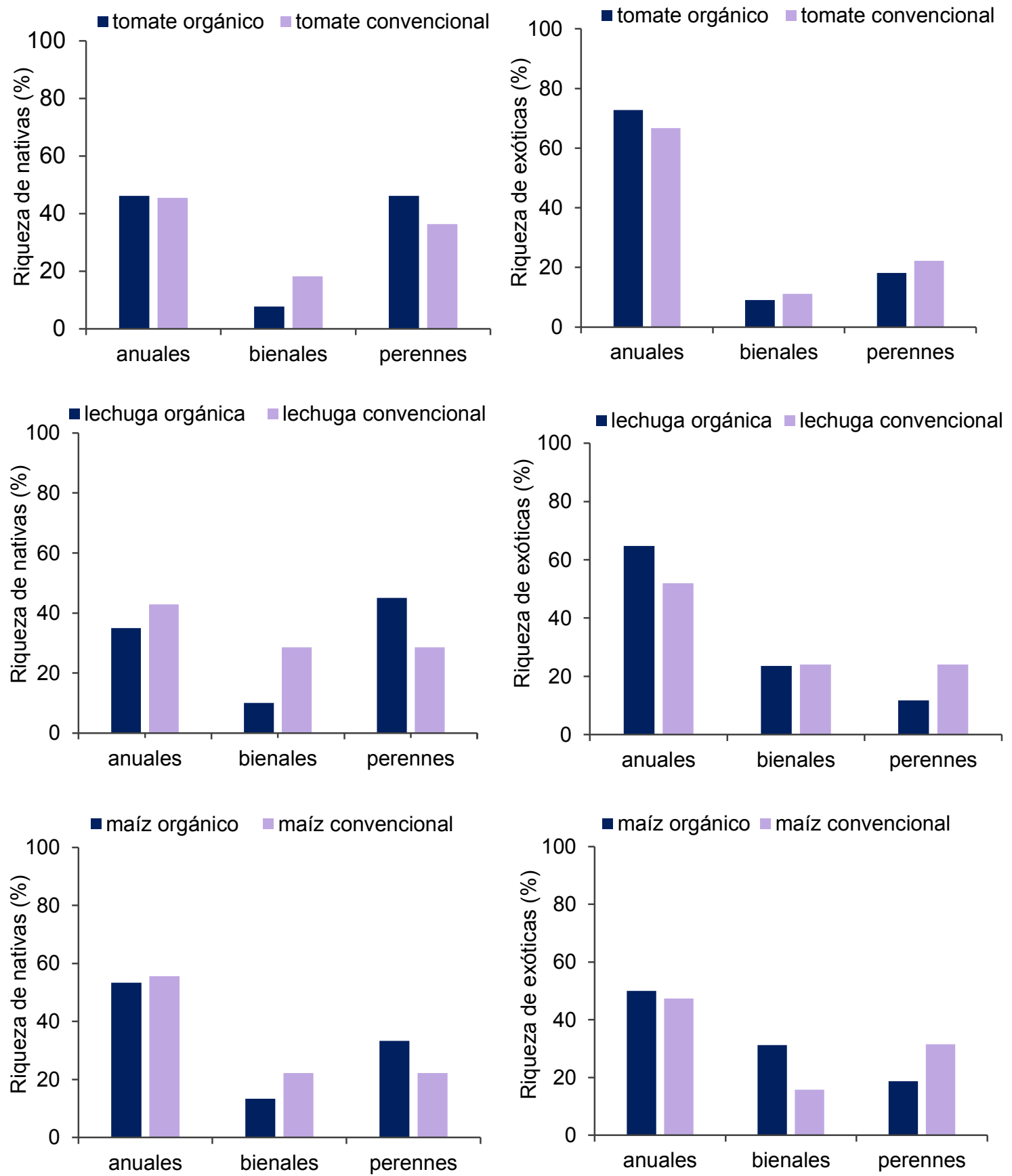

Figura 5.6. Proporción de especies de plantas espontáneas nativas (izquierda) y exóticas (derecha) clasificadas según el ciclo de vida y la morfología en cultivos de tomate, lechuga y maíz, orgánicos y convencionales intensivos.

Diversidad Vegetal Espontánea en Agroecosistemas Hortícolas de La Plata y su relación con diferentes estilos de Agricultura: Importancia 
Las bienales se relacionaron de manera diferente entre cultivos y manejos. Las exóticas presentaron, respecto de las nativas, mayores diferencias entre la proporción de especies de ciclo de vida corto con las de ciclo largo, siendo las anuales más abundantes (como lo indica la diferencia entre las barras de anuales y perennes).

\subsubsection{Diversidad Alfa en función de las prácticas de manejo}

Cuando se analizaron los tres tipos de manejo juntos integrando las modalidades a campo e invernáculo se encontró que la fertilización química, la intolerancia a la presencia de malezas y el desmalezado químico fueron las variables que se correlacionaron en forma significativa y de manera inversa con la riqueza de especies (Tabla 5.2). Esto significa que la riqueza disminuye a medida que aumenta el uso de fertilizantes nitrogenados, de herbicidas (frecuencia de aplicación y espectro de acción) y la intolerancia del agricultor hacia la presencia de espontáneas (bajo umbral de malezas). Como lo indican los índices de Shannon (Tabla 5.2), la diversidad se correlacionó de manera significativa e inversa con el desmalezado químico principalmente, seguido de la fertilización química. Esto significa que, al igual que la riqueza, la diversidad disminuye con el aumento de la intensidad de uso de estos insumos.

TABLA 5.2. Valores de correlación entre las variables de manejo y la riqueza de especies de plantas espontáneas por cultivo $(n=62)$, bajo manejo orgánico, bajos insumos e intensivos incluyendo la modalidad a campo e invernáculo juntas. El asterisco $\left({ }^{*}\right)$ indica los valores significativos, $(p \leq 0.05)$.

\begin{tabular}{lll}
\hline Variable de manejo & Riqueza & Diversidad \\
\hline Fertilización química & $-0,508^{*}$ & $-0.341^{*}$ \\
Desmalezado químico & $-0,367^{*}$ & $-0,417^{*}$ \\
Intolerancia a las malezas & $-0,318^{*}$ & $-0,156$ \\
Intensidad de uso del suelo & $-0,195$ & $-0,088$ \\
Historia de uso & $-0,131$ & $-0,135$ \\
Riego & $-0,052$ & $-0,187$ \\
Ausencia de rotaciones & $-0,026$ & $-0,076$ \\
Esterilizadores & $-0,001$ & -0.166 \\
\hline
\end{tabular}

Cuando se analizaron la modalidades a campo e invernáculo por separado, se encontró que, en los cultivos a campo, la fertilización química $(r=-0,501 ; p \leq 0,05)$ y el desmalezado químico $(r=-0,363 ; p \leq 0,05)$ fueron las variables que más se correlacionaron (de manera inversa) con la riqueza de especies espontáneas. La

Diversidad Vegetal Espontánea en Agroecosistemas Hortícolas de La Plata y su relación con diferentes estilos de Agricultura: Importancia 
siguiente variable fue la intolerancia a las malezas aunque no fue significativa. La fertilización ( $r=-, 0342 p \leq 0,05)$ y el desmalezado químico $(r=-0,335 ; p \leq 0,05)$ se asociaron también negativamente con la diversidad. En la producción bajo invernáculo la fertilización fue la variable de valor más alto que se correlacionó de manera inversa con la riqueza y la diversidad aunque no fue significativa ( $r=-0,33$ vs $r=-0,25 ; p \leq 0,05$ ). En los distintos análisis, la fertilización química fue la variable que mostró una mayor influencia sobre la riqueza de plantas a escala parcelas de cultivo. A su vez, todas las variables identificadas como relevantes, no tuvieron valores tan altos de correlación $(r<$ $0,6)$.

\subsection{DISCUSIÓN}

\subsubsection{Diversidad dentro de las parcelas de cultivos bajo diferente manejo}

Los resultados encontrados muestran la influencia que tiene el manejo sobre la riqueza y diversidad de plantas en los cultivos del Cinturón Hortícola. La tendencia a una mayor riqueza y diversidad encontrada en los sistemas orgánicos confirma la primer hipótesis y coincide con los resultados de numerosos trabajos (Gabriel, 2010; Henckel et al., 2015; Edesi et al., 2012). En los resultados de un metanálisis de Bengtsson et al., (2005), la agricultura orgánica incrementó la diversidad especialmente en la escala de lote o parcela (plot o field). Esto refleja la importancia de analizar la diversidad Alfa a escala parcela de cultivo.

Cuando se comparan los mismos cultivos bajo diferente manejo, la riqueza promedio de especies (entre 15 y 20 especies) fue más alta en los cultivos orgánicos que en los convencionales intensivos (Figura 5.1). Para los distintos manejos, la riqueza fue superior a lo encontrado en cultivos extensivos de la Pampa Ondulada, cuyo valor medio fue de 8 especies (Poggio et al., 2013). De la misma manera, los valores promedios del índice de Shannon resultaron elevados (entre 3 y 3,5) comparados con otros estudios en los que se encontraron valores cercano a 1 y hasta 2,5 (Edesi et al., 2012; Purichelli et al., 2012; Bavec \& Bavec, 2015). Generalmente, en sistemas naturales los índices alcanzan valores máximos de 4,5 (Magurran, 1998). Estos aspectos indicados muestran que los cultivos del Cinturón Hortícola favorecen una importante diversidad de especies espontáneas. El valor fue superior en los cultivos orgánicos respecto de los convencionales ya que presentaron una mayor variedad de 
especies y, a grandes rasgos, una distribución más equitativa de su abundancia, coincidiendo con otros trabajos (Hyvönen et al., 2002, 2003; Gabriel et al., 2010; Henckel et al., 2015). La mayor diversidad podría deberse a la percepción que tiene el agricultor acerca de las plantas espontáneas, lo que influye en la presión de control de las mismas. El agricultor orgánico es más tolerante a la presencia de especies espontáneas y ejerce solo un control mecánico, no selectivo y no utiliza fertilizantes químicos, favoreciendo la mayor cobertura de especies de similar abundancia relativa. Esa menor presión en el manejo de las plantas no cultivadas podría favorecer una diversidad mayor, al mantener niveles de disturbios menores que actúan sobre la biomasa de las plantas. En el caso del cultivo de tomate, los distintos agricultores manifestaron mantener un mayor cuidado de ese cultivo por su valor económico. Tanto en su versión convencional como orgánica, la intensificación del control (respectivamente: método químico vs mecánico principalmente) condujo a valores similares de diversidad entre los distintos manejos, aunque la variabilidad fue mayor en cultivos orgánicos.

La variabilidad encontrada en los valores de los índices en un mismo cultivo, podría deberse a diferencias en la cobertura vegetal de las parcelas relevadas a la fecha de muestreo, debido a las variantes de labores de control realizadas y en períodos de tiempo diferentes.

Los cultivos de características estructurales distintas respondieron en termino relativos de la misma manera con la intensidad de manejo en los componentes de la diversidad. Algunas de las diferencias que se observaron probablemente estén debidas al efecto de la estructura de cada uno de esos cultivos.

\subsubsection{Composición florística de los cultivos bajo diferente manejo}

En el análisis de Clúster, las parcelas de cultivo con similar manejo tendieron a agruparse por la mayor similitud en la composición florística, lo que es consistente con la primera predicción (hipótesis 2). Las parcelas convencionales de bajos insumos se parecieron menos entre sí y se vincularon con distinta semejanza a los otros manejos. Esto podría deberse a las características de estos agricultores que utilizan prácticas que son compartidas con uno u otro tipo, como el uso o no de herbicidas y/o fertilizantes, sujetos a la situación económica del momento que influye sobre la posibilidad de acceso a los insumos, sin adoptar estrictamente un "paquete tecnológico" (Capítulo 4). Por lo 
tanto, el manejo de los cultivos dentro de esta categoría puede ser más variable generando diferencias en la composición de especies que los asemejan a un u otro tipo de forma de producción.

La comparación de las distancias entre los grupos de muestras que reúnen diferentes manejos en el análisis de Cluster (Figura 5.4) no permite aseverar firmemente la segunda predicción (hipótesis 2), que plantea que la composición florística se vuelve más semejante con el aumento de la intensidad de manejo. Esto podría deberse al mayor impacto en el índice en muestras de menor riqueza en los cultivos convencionales, que dejan pobremente reflejada la supuesta homogeneidad en la composición de especies en este tipo de manejo.

Por otro lado, el importante número de especies comunes asemejaría a los tipos de manejos en la composición florística. Sin embargo, los cultivos menos intensivos se destacaron por la presencia de especies exclusivas, por especies comunes de mayor frecuencia y una tendencia a una mayor abundancia en general (Tabla 5.1). Esto se corresponde con la mayor riqueza y diversidad promedio encontrada en los cultivos de manejo a campo y la diferente lógica del agricultor en el manejo de las plantas espontáneas. Esto coincide con los resultados de otro estudio (Edesi et al., 2012) y sugiere una composición florística más heterogénea a nivel Alfa en las fincas orgánicas, de acuerdo con lo planteado en la segunda hipótesis.

En el tomate convencional, al considerar las modalidades a campo e invernáculo juntas, se destacó la presencia de especies de baja frecuencia. Estas especies se encontraron en muestras de invernáculo y principalmente en los surcos. Esto podría deberse a las diferencias en las condiciones ambientales respecto del campo que permiten la aparición de especies eventuales favorecidas por las condiciones ideales y controladas del cultivo bajo cubierta, y que escapan al manejo más intensivo. Por ejemplo situadas por fuera de los lomos donde se aplica Bromuro de Metilo.

Los resultados muestran que la homogeneidad esperada en las parcelas de cultivos más intensivos se refleja más en la semejanza composicional que en un efecto de dominancia de especies, que no es tan visible debido a que las plantas presentan, en general, valores bajos de abundancia, si bien la equitabilidad tiende a bajar con el aumento de la intensidad de manejo. 


\subsubsection{Composición de especies según los grupos funcionales}

En los cultivos orgánicos y convencionales se encontró una mayor proporción de especies exóticas, anuales y dicotiledóneas, lo que caracteriza a los sistemas que presentan un régimen de disturbio periódico en donde se establecen especies pioneras en sucesiones secundarias y donde existe gran disponibilidad de recursos (Zanin et al., 1997; Suárez et al. 2001; Urban; 2010). Esto coincide con otros estudios en trabajos en sistemas extensivos y hortícolas convencionales (Poggio, 2005; de la Fuente et al, 2006; Cerazo \& Conticello, 2008; Rassam, 2011; Poggio et al., 2013). Sin embargo, los cultivos orgánicos presentaron una mayor proporción de especies nativas y de ciclo de vida largo, como las perennes. Estas características funcionales sugieren que los cultivos orgánicos se asocian a sistemas de menor disturbio respecto de los convencionales (Travlos et al., 2018). Esto confirma que la intensidad y duración de las técnicas aplicadas en cada tipo de manejo, afectan la composición estructural de grupos funcionales de plantas espontáneas, como se predijo (hipótesis 3).

Algunos autores proponen que a altas dosis de fertilizantes, los pastos anuales exóticos se reproducen mejor comparado con las especies nativas y estas últimas disminuyen su riqueza (Travlos et al., 2018). Por otro lado, la tendencia a un mayor uso de herbicidas promueve una mayor diversidad de monocotiledóneas resistentes y una reducción de especies de hoja ancha (Shepherd et al., 2003; Hyonen et al., 2003). Esto se observó en el cultivo de maíz convencional. Sin embargo, en la lechuga la proporción de monocotiledóneas fue mayor en los cultivos orgánicos que en los convencionales y en el tomate presentó menores diferencias. Esto podría deberse a diferencias en el manejo de cada cultivo. Por ejemplo, la lechuga es un cultivo de hoja que suele ser desmalezado para simplificar la cosecha y la venta con lo cual el efecto de disturbio entre ambos manejos podría ser similar, favoreciendo la presencia de monocotiledóneas, independientemente del método utilizado.

\subsubsection{Diversidad Alfa en función de las prácticas de manejo}

Tal como se predijo (hipótesis 4), la riqueza de espontáneas disminuyó con el incremento en el uso de herbicidas y con la menor cobertura de espontáneas tolerada por el agricultor (bajo umbral de malezas). Sumado a esto, el uso de fertilizantes químicos de base nitrogenada fue la variable que expresó el mayor efecto negativo sobre la riqueza. 
La tendencia a un mayor uso de fertilizantes nitrogenados, herbicidas y a menor umbral de malezas se correspondió con un manejo convencional intensivo, como se indicó en el Capítulo 4. Por lo tanto, la riqueza a escala parcela de cultivo disminuye con la intensidad de estas prácticas de manejo. Por el contrario, aumenta cuando no se utilizan estos insumos y cuando el nivel de plantas espontáneas tolerado es mayor, como en el caso de los agricultores orgánicos.

En los sistemas extensivos, ciertos antecedentes muestran que el uso de fertilizantes químicos modifica las relaciones de competencia entre el cultivo y la vegetación espontánea (Bengtsson et al., 2005). El crecimiento del follaje del cultivo, favorece la dominancia de especies tolerantes a condiciones de poca luz y de alta disponibilidad de nitrógeno (Ying et al., 2006; Pysek \& Leps, 1991; Tang et al., 2013). Esto podría corresponderse con los bajos valores de riqueza e índices de Shannon encontrados en los sistemas intensivos, indicando una disminución en la equitabilidad a medida que se incrementa el uso de fertilizantes. Pysek \& Leps (1991) encontraron que a alta dosis de fertilizantes el efecto de disminuir la diversidad fue mayor que en dosis pequeñas (140 Kg de N.ha- ${ }^{1}$.año- ${ }^{1}$ ). En la región, se aplican dosis preestablecidas sin considerar la potencialidad productiva del suelo superiores a $300 \mathrm{Kg} \mathrm{N}$.ha- ${ }^{1}$.año- ${ }^{1}$ en forma de fertilizantes sintéticos (Alconada et al., 2011). En este estudio las mayores dosis encontradas fueron de $450 \mathrm{Kg} \mathrm{N}$.ha- ${ }^{-1}$.año- ${ }^{1}$ correspondiendo a los sistemas convencionales, cuando en realidad no se recomienda superar las $350 \mathrm{Kg} \mathrm{N}$.ha- ${ }^{1}$. año-1. Este exceso de fertilizantes sugiere un importante riesgo sobre la conservación de las especies, especialmente en cultivos bajo manejo intensivos. Si bien en el invernáculo el valor de correlación de la fertilización fue cercano al del manejo a campo, no fue significativo ( $r=0,33$ a campo; $r=0,36$ a invernáculo). Esto podría deberse a que el menor número de muestras condicionan el análisis de correlación entre las variables.

La restricción de uso de herbicidas en los orgánicos podría contribuir a una mayor diversidad de espontáneas respecto de los convencionales (Gabriel et al., 2010; Roschewitz et al. 2005; Rundlöf et al., 2012). Si bien los sistemas de agricultura moderna son vulnerables a la invasión de espontáneas debido a la frecuencia e intensidad de disturbio (Martínez-Ghersa et al., 2000), la pérdida de especies causada por el uso de herbicidas tales como el glifosato, puede superar al efecto de recolonización (Vitta et al., 2004). Los herbicidas pueden ser uno de los filtros más importantes porque pueden ser selectivos, resultan en una alta mortalidad en individuos susceptibles y pueden ser 
residuales (Légère et al., 2005; Ryan et al., 2010). En la zona estudiada, el uso más intensivo de herbicidas se debió a la utilización de productos de amplio espectro y de mayor frecuencia de aplicación como los herbicidas totales de principios activos paraquat y glifosato, y selectivo de amplio espectro como metribuzín y s-metacloro (ver Capítulo 4). Esto indica el importante efecto negativo de los herbicidas sobre la riqueza de espontáneas, coincidiendo con otros estudios (Mc Laughlin \& Mineau, 1995; Ghersa \& Martínez- Ghersa, 2000).

En los cultivos bajo invernáculo, la relación entre la riqueza de especies y los herbicidas no fue significativa. Esto puede deberse a que los herbicidas se utilizan, principalmente, en cultivos al aire libre convencionales. En cambio, en el invernáculo los herbicidas caracterizan sólo a los manejos de bajos insumos, siendo en los intensivos más frecuente el uso de esterilizadores (bromuro de metilo). Por lo tanto, al ser menos representativos los casos que emplean herbicidas en cultivos bajo cubierta, podrían no ser detectados en la correlación como una variable significativa.

Los resultados señalados anteriormente indican que la diversidad Alfa varía según el manejo que se realice, tanto a nivel de los insumos utilizados y lógica del agricultor. En las parcelas cultivadas orgánicas, la ausencia de herbicidas y de fertilizantes nitrogenados sintéticos y, la mayor tolerancia a las espontáneas por parte del agricultor, promueven una flora de mayor riqueza y diversidad respecto de las convencionales. A su vez, se destacan los grupos funcionales vinculados a niveles de disturbio intermedios, como las especies de nativas y bienales-perennes. Por lo tanto, un manejo menos intensivo en las parcelas de cultivos contribuye a mantener una diversidad de especies espontáneas y una composición florística y funcional más rica a nivel Alfa.

\subsection{CONCLUSIONES}

Los resultados presentados en este capítulo confirman que la diversidad a escala Alfa disminuye con el aumento de la intensidad de las prácticas de manejo.

Los cultivos orgánicos presentaron una mayor riqueza, equitabilidad y diversidad de especies espontáneas que los convencionales. Se caracterizaron por una mayor cobertura de espontáneas y de abundancias similares por especie, debido a una mayor

Diversidad Vegetal Espontánea en Agroecosistemas Hortícolas de La Plata y su relación con diferentes estilos de Agricultura: Importancia 
tolerancia a la presencia de dicha vegetación por parte de los agricultores, lo que influye en las decisiones de control.

Los cultivos orgánicos, presentaron una composición florística y funcional más rica, donde se destacó la presencia de especies exclusivas y comunes frecuentes, especies nativas y de ciclo de vida largo. Esto se corresponde con las características de sistemas productivos menos intensivos, de bajo disturbio, que posibilitan la heterogeneidad intraparcela.

Por el contrario, el aumento de la intensidad de las prácticas de manejo se correspondió con una menor diversidad de especies en los cultivos convencionales, que resultó en una composición florística y funcional más homogénea.

El uso de fertilizantes sintéticos nitrogenados, herbicidas y una menor tolerancia a la presencia de vegetación espontánea tuvieron efecto sobre la diversidad Alfa en los cultivos bajo diferentes estilos de agricultura. El uso de fertilizantes nitrogenados fue la variable más asociada negativamente con la riqueza y diversidad, lo que se explica por el efecto de modificar las relaciones de competencia con el cultivo, favoreciendo la presencia de especies dominantes. Esto se corresponde con la aplicación de altas dosis de este insumo en la zona estudiada.

Los resultados presentados de este capítulo permiten concluir que la tendencia al empleo de prácticas estandarizadas que se repiten en el tiempo, como los productos sintéticos de uso generalizado (herbicidas, fertilizantes), tienden a homogeneizar el hábitat cultivado. Por lo tanto, constituyen un riesgo para el mantenimiento de la biodiversidad y sus funciones ecológicas asociadas.

Otros factores asociados a otras escalas espaciales podrían tener influencia en la diversidad. Por lo tanto, en el siguiente capítulo se analiza el efecto de la heterogeneidad de hábitats cultivados sobre la diversidad en las escalas Beta y Gamma.

\subsection{BIBLIOGRAFÍA}

Alconada M, Cuellas M, Poncetta P, Barragán S, Inda E \& Mitidieri A. 2011. Fertirrigación en cultivo de tomate protegido: Nutrición nitrogenada. Efectos en el suelo y en la producción. Horticultura Argentina 30(72): 5-13.

Diversidad Vegetal Espontánea en Agroecosistemas Hortícolas de La Plata y su relación con diferentes estilos de Agricultura: Importancia 
Andersson TN \& Milberg P. 1998. Weed flora and the relative importance of site, crop, crop rotation, and nitrogen. Weed Science 46: 30-38.

Altieri MA. 1999. The ecological role of biodiversity in agroecosystems. Agriculture, Ecosystems and Environment 74: 19-31.

Bárberi P, Burgio G, Dinelli G, Moonen AC, Otto S, Vazzana C \& Zanin G. 2010. Functional biodiversity in the agricultural landscape: relationships between weeds and arthropod fauna. Weed Research 50: 388-401.

Batáry P, Baldi A, Kleijn D, \& Tscharntke T. 2011. Landscape-moderated biodiversity effect of agri-enviromental management: a meta-analysis. Proceeding of the Royal Society 278 : 1894-1902.

Bavec M \& Bavec F. 2015. Impact of organic farming and biodiversity. En: Blanco J \& Yueh-Hisin Lo \& Roy S (Eds.). Biodiversity and ecosystems. Linking structure and function. Intech $658 \mathrm{pp}$.

Bengtsson J, Ahnstrom J \& Weibull AC. 2005. The effects of organic agriculture on biodiversity and abundance: a meta-analysis. Journal of Applied Ecology 42: 261269.

Benton TG, Vickery JA \& Wilson JD. 2003. Farmland biodiversity: is habitat heterogeneity the key? Trend in ecology and evolution 18(4): 182-188.

Censo Hortiflorícola de Buenos Aires 2005 (CHFBA'05). 2006. Ministerio de Asuntos Agrarios y Ministerio de Economía de la Provincia de Buenos Aires. 115 p.

Cerazzo B \& Conticello L. 2008. Comunidades de malezas en cultivos en la provincia de Neuquén (Argentina). Boletín de la Sociedad Argentina de Botánica 43: 121-135.

Collins W \& Qualset O.1999. Biodiversity in Agroecosystems CRC Press, Boca Raton, Florida. $334 \mathrm{p}$.

MacArthur D. 1965. Patterns of species diversity. Biological Review 40: 510-533.

Magurran AE. 1988. Ecological Diversity and Its Measurement. Pinceton University Press, New Jersey. 179 p.

Marshall EJP, Brown VK, Boatman ND \& Lutman PJW. 2003. The role of weeds in supporting biological diversity within crop fields. Weed Research 43: 77-89.

McLaughlin A \& Mineau P. 1995. The impact of agricultural practices on biodiversity. Agriculture, Ecosystems \& Environment 55: 201-212.

de la Fuente EB, Suarez SA, Ghersa CM \& León RJC. 1999. Soybean weed comunities: relationships whit cultural history and crop yield. Agronomy Journal 91: 234-241.

de la Fuente EB, Suarez SA \& Ghersa CM. 2006. Soybean weed community composition and richness between 1995 and 2003 in the Rolling Pampas (Argentina). Agriculture, Ecosystems and Environment 115: 229-236.

Diversidad Vegetal Espontánea en Agroecosistemas Hortícolas de La Plata y su relación con diferentes estilos de Agricultura: Importancia 
Diamond JM. 1975. Assembly of species communities. Ecology and evolution of communities. En: Cody ML \& Diamond JM (Eds.). Harvard University Press, Cambridge, USA. 342-444 p.

Edesi L, Jarvan M, Adamson A, Lauringson E. \& Kuht J. 2012. Weed species diversity and community composition in conventional and organic farming: a five-year experiment. Zemdirbyste-Agriculture 99: 339-346.

Fischer J, Abson, DJ, Butsic V, Chappell MJ, Ekroos J, Hanspach, J \& Wehrden H. 2014. Land sparing versus land sharing: moving forward. Conservation Letters 7(3): 149157.

Fried G, Norton LR \& Reboud X .2008. Environmental and management factors determining weed species composition and diversity in France. Agriculture, Ecosystems \& Environment 128: 68-76.

Gabriel D, Sait SM, Hodgson JA, Schmutz U, Kunin WE \& Benton TG. 2010. Scale matters: the impact of organic farming on biodiversity at different spatial scales. Ecology Letters 13(7): 858-869.

Ghersa CM \& Martínez-Ghersa MA. 2000. Ecological correlates of weed seed size and persistence in the soil under different tilling systems: implications for weed management. Field Crops Research 67: 141-148.

Ghorbanali R, Lafiti N, Soltani A \& Kamkar B. 2011. Impact of crop management on weed species diversity and community composition of winter wheat field in Iran. Weed Biology and Management 11: 83-90.

Henckel L, Borger L, Meiss H, Gaba S \& Bretagnolle V. 2015. Organic field sustain weed metacommunity in farmland landscapes. Proceeding of the Royal Society B: 282: 20150002.

Hill MO. 1979. TWISPAN-A FORTRAN program for arranging multivariate data in an ordered two-way table by classifi cation of the individuals and attributes. Cornell University, Nueva Jersey, EEUU.

Hole DG, Perkins AJ, Wilson JD, Alexander IH, Grice PV \& Evans AD. 2005. Does organic farming benefit biodiversity? Biological Conservation 122:113-130.

Hyvönen T \& Salonen J. 2002. Weed species diversity and community composition in cropping practices at two intensity levels - a six-year experiment. Plant Ecology 159: 73-81.

Hyvönen T, Ketoja E, Salonen J, Jalli H \& Tiainen J. 2003. Weed species diversity and community composition in organic and conventional cropping of spring cereals. Agriculture, Ecosystems and Environment 97: 131-149.

Kleijn, D, Kohler F, Báldi A ,Batáry P, Concepción ED, Clough Y, Díaz M, Gabriel D, Holzchuh A, Knop E, Kovács A, Marshall EJP, Tscharnke T \& Verhults J. 2009. On the relationship between farmland biodiversity and land-use intensity in Europe. Proceedings of the Royal Society B: 276: 903-909. 
Krebs JR, Wilson JD, Bradbury RB \& Siriwardena GM. 1999. The second silent spring? Nature 400: 611-612.

Laird RA \& Champ BS. 2006. Competitive intransitivity promotes species coexistence. The American Naturalist 168(2): 182-193.

Légère A, Stevenson FC \& Benoit DL. 2005. Diversity and assembly of weed communities: contrasting responses across cropping systems, Weed Research 45: 303-315.

Marshall EJP, Brown VK, Boatman ND, Lutman PJW \& Squire GR. 2002. The impact of herbicides on weed abundance and biodiversity. DEFRA, Informe PN0940.

Martínez-Ghersa MA, Ghersa CM \& Satorre EH. 2000. Coevolución of agriculture systems and their weed companions: implications for research. Field Crops Research 67: 181-190.

Matteucci, SD \& Colma A. 1982. Metodología para el estudio de la vegetación. Monografía $\mathrm{n}^{0} 22$, Serie de biología de la OEA.

Matthew R, Ryan RG, Smith, SB. Mirsky DA Mortensen \& Seidel R. 2010. Management Filters and Species Traits: Weed Community Assembly in Long-Term Organic and Conventional Systems. Weed Science 58: 265-277.

McLaughlin A \& Mineau P. 1995. The impact of agricultural practices on biodiversity. Agriculture, Ecosystems \& Environment 55: 201-212.

Pal RW, Pinke G, Botta-Dukat Z, Campetella G, Bartha S, Kalocsai R \& Lengyel A. 2013. Can management intensity be more important than environmental factors? A case study along an extreme elevation gradient from central Italian cereal fields. Plant Biosystems 147: 343-353.

Pysek P \& Leps J. 1991. Response of weed community to nitrogen fertilization: a multivariate analysis. Journal of Vegetation Science 2: 237-244.

Poggio SL. 2005. Structure of weed communities occurring in monoculture and intercropping of field pea and barley. Agriculture, Ecosystems and Environment 19: 48-58.

Poggio SL. 2012. Cambios florísticos en comunidades de malezas: un marco conceptual basado en reglas de ensamblaje. Ecología Austral 22: 150-158.

Poggio SL, Chaneton EJ \& Ghersa CM. 2013. The arable plant diversity of intensively managed farmland: Effects of field position and crop type at local and landscape scales. Agriculture, Ecosystems \& Environment 166: 55-64.

Puricelli E, Faccini D, Nisenshon L \& Tuesca D. 2012. Weed cover, frequency and diversity in field plots and edges in the soybean central region of Argentina. Agricultural Science 3(5): 631-639.

Rassam G, Lafiti N, Soltani A \& Kamkar B. 2011. Impact of crop management on weed species diversity and community composition of winter wheat fields in Iran. Weed Biology and Management 11: 83-90.

Diversidad Vegetal Espontánea en Agroecosistemas Hortícolas de La Plata y su relación con diferentes estilos de Agricultura: Importancia 
Roig FA. 1973. El cuadro fitosociológico en el estudio de la vegetación. Deserta 4: 4567.

Roschewitz I, Gabriel D, Tscharntke T \& Thies C. 2005. The effects of landscape complexity on arable weed arable diversity in organic and conventional farming. Journal of Applied Ecology 42(5): 873-882.

Rundlöf M, Edlund M \& Smith HG. 2010. Organic farming at local and landscape scales benefits plant diversity. Ecography 33: 514-522.

Ryan MR, Smith RG, Mirsky SB, Mortensen DA \& Seidel R. 2010. Management filters and species traits: weed community assembly in long-term organic and conventional systems. Weed Science 58: 265-277.

Servicio Nacional de Sanidad y Calidad Agroalimentaria (SENASA). 2016. Casos confirmados de malezas. Disponible en: http://www.senasa.gob.ar/casosconfirmados-de-malezas-resistentes-en-argentina. Ultimo ingreso: 7/5/2018.

Shepherd M, Pearce B, Cormack B, Phillipps L, Cuttle S, Bhogal A, Costigan P \& Unwin R. 2003. An assessment of the environmental impacts of organic farming. Una revisión para Defra-funded project OF0405. ADAS Consultora Ltd. Mansfield. 80 pp. Disponible en: http://orgprints.org/6784/2/OF0405_909_TRP.pdf.

Stupino SA, Frangi JL, Sarandón SJ, Arturi MF \& Ferreira AC. 2008. Plant diversity in two farms under organic and conventional management in La Plata, Argentina. A case study. Revista Brasilera de Agroecología, Asociación Brasilera de Agroecología 3(3): 24-35.

Suárez SA, de la Fuente EB, Ghersa CM \& León RJC. 2001. Weed community as an indicator of summer crop yield and site quality. Agronomy Journal 93: 524-530.

Sutcliffe OL \& Kay QON. 2000. Changes in the arable flora of central southern England since the 1960s. Biological Conservation 93:1-8.

Swift MJ, Izac MN \& van Noordwijk M. 2004. Biodiversity and ecosystem services in agricultural landscapes-are we asking the right questions? Agriculture, Ecosystems and Environment 104: 113-134.

Tang L, Wan K, Chuanpeng C \& Chen F. 2013. Effect of fertilization patterns on the assemblage of weed communities in an upland winter wheat field. Journal of Plant Ecology 7(1): 39-50.

Terradas J. 2001. De la ecofisiología de las plantas a la dinámica de comunidades y paisajes. Omega, Barcelona. 760 pp.

Travlos IS, Cheimona N, Roussis I \& Bilalis DJ. 2018. Tillage and fertilization effect on weeds. Frontiers in Environmental Science 11(6): 1-10.

Tuck SL, Winqvist C, Mota F, Ahnström, J, Turnbull, LA \& Bengtsson J. 2014. Land-use intensity and the effects of organic farming on biodiversity: a hierarchical metaanalysis. Journal of Applied Ecology 51: 746-755. 
UNEP. 2000. The Biodiversity Agenda. Decisiones adoptadas por la conferencia de las partes en el convenio sobre la diversidad biológica en su quinta reunión. Apéndice Nairobi, 15-26 de Mayo de 2000.

Urban S. 2010. Synanthropic vegetation: pattern of varius disturbances on life history traits. Acta Botanica Croata 69(2): 215-227.

van Elsen T. 2000. Species diversity as a task for organic agriculture in Europe. Agriculture, Ecosystems and Environment 77: 101-109.

Vita JI, Tuesca D \& Puricelli E. 2004. Widespread use of glyphosate tolerant soybean and weed community richness in Argentina. 2004. Agriculture, Ecosystems and Environment 103: 621-624.

Whittaker RH. 1977. Evolution of species diversity in land communities. Evolutionary Biology 10: 1-67.

Yin L, Cai Z \& Zhong W. 2006. Changes in weed community diversity of maize crops due to long-term fertilization. Crop Protection 25(9): 910-4.

Zanin G, Otto S, Riello L \& Borin M. 1997. Ecological interpretation of weed flora dynamics under different tillage systems. Agriculture, Ecosystems and Environment 66: 177-188. 


\section{CAPÍTULO 6}

ANÁLISIS DE LA RELACION ENTRE LA HETEROGENEIDAD DE HÁBITATS Y LA DIVERSIDAD DE PLANTAS ESPONTÁNEAS EN FINCAS BAJO DIFERENTE MANEJO. ESCALAS DE ANÁLISIS BETA, GAMMA Y PAISAJE.

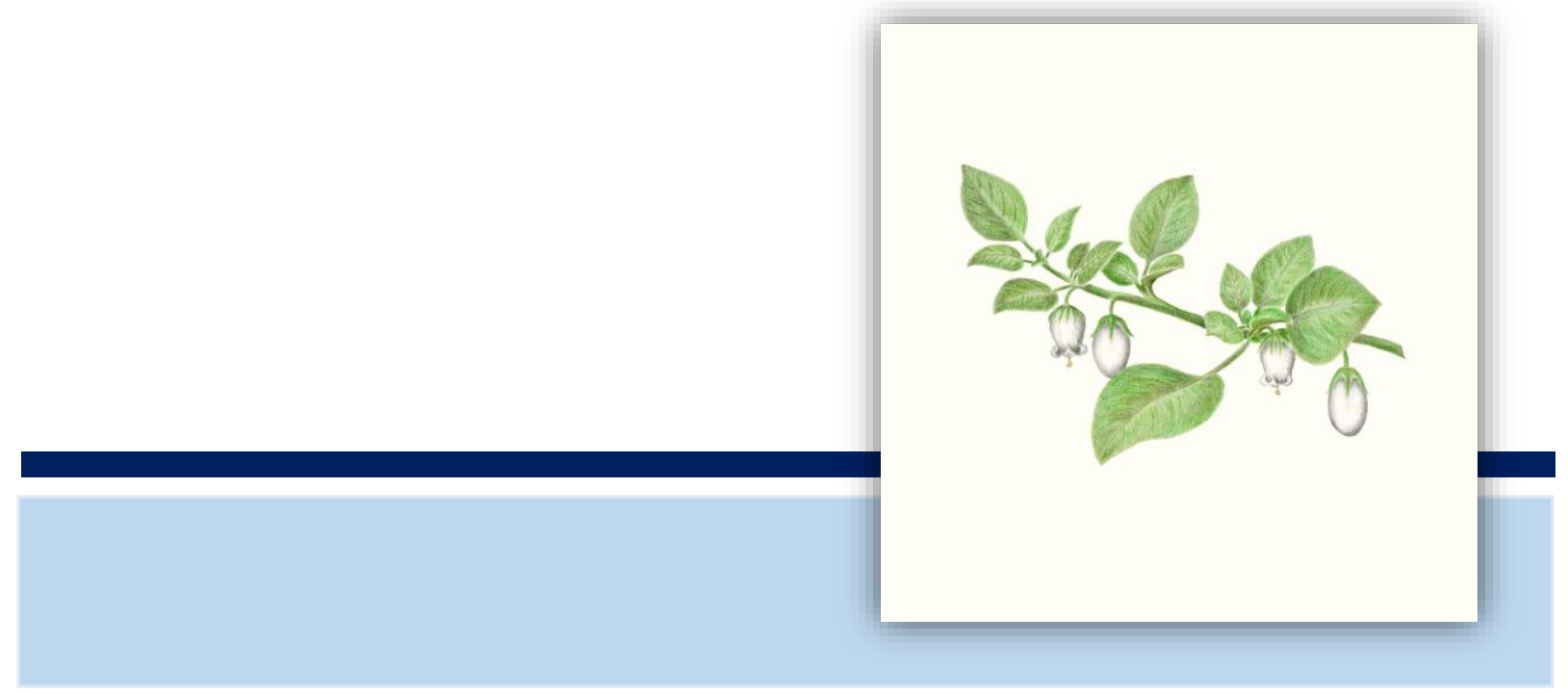




\section{CAPÍTULO 6}

La mitad de la belleza depende del paisaje y la otra mitad del hombre que la mira.

Lyn Yutang (1895-1976). Escritor.

\section{ANÁLISIS DE LA RELACIÓN ENTRE LA HETEROGENEIDAD DE HÁBITATS Y LA DIVERSIDAD DE PLANTAS ESPONTÁNEAS EN FINCAS BAJO DIFERENTE MANEJO. ESCALAS DE ANÁLISIS BETA, GAMMA Y PAISAJE.}

\subsection{INTRODUCCIÓN}

Uno de los efectos de la intensificación de la agricultura ha sido la disminución de la heterogeneidad de hábitats (Benton et al., 2003; Foley et al., 2005; Tscharntke et al., 2005), lo que ha generado la pérdida de biodiversidad tanto a nivel finca como de paisaje (Altieri, 1999; Sarandón, 2002; SCDB, 2008). Esto representa la principal causa de la disminución en la riqueza de especies espontáneas que tienen una función relevante en sostener toda la diversidad biológica asociada a los cultivos (Krebs et al., 1999; Sutcliffe \& Kay, 2000; van Elsen, 2000; Benton et al., 2003; Marshall et al., 2003; Jackson et al., 2007).

A escala de finca se observan comúnmente pocas especies de cultivos, a través de prácticas de manejo sincrónicas y estandarizadas que favorecen la simplificación del agroecosistema (Evans, 1993; Matson et al., 1997; Gliessman, 2001; Benton et al., 2003; Swift et al., 2004).

Además del efecto de las prácticas de manejo sobre la vegetación, la diversidad local de especies espontáneas puede estar influenciada por el paisaje (Forman, 1995; Weibull \& Ostman, 2003; Roschewitz et al., 2005; Tscharntke et al., 2005; Henckel et al., 2015). La ocupación de un sitio disturbado puede depender, en parte, de propágulos que provienen de otros hábitats circundantes que actúan como refugios (Terradas, 2001; Weibull \& Ostman, 2003; Roschewitz et al., 2005). Los paisajes más complejos o heterogéneos 
pueden contener un mayor número de especies que los paisajes simples, al presentar una mayor diversidad de hábitats naturales y cultivados alternativos que pueden actuar como fuente de propágulos para las fincas (Benton et al., 2003; Tscharntke et al., 2005). Por lo tanto, la complejidad del paisaje puede mitigar la eliminación local de espontáneas a través del aporte de propágulos desde las fuentes externas de dispersión (Roschewitz et al., 2005).

La heterogeneidad ha sido uno de los principales determinantes de la biodiversidad a diferentes escalas espaciales (Shmida \& Wilson, 1985). Una de las escalas es la denominada diversidad Beta $(\beta)$, que es la tasa de recambio de especies entre distintos hábitats y describe que tan diferente (o similar) es un rango de hábitats o muestras en términos de su composición específica. Otra escala de diversidad es Gamma $(\gamma)$, que es la riqueza total de especies presentes en un área determinada. La definición de las escalas varía según el grupo de estudio y de los objetivos del trabajo (Shmida \& Wilson, 1985; Whittaker et al., 2001; Koleff \& Gaston, 2002; Roschewitz et al., 2006). En este sentido, el estudio de las relaciones entre los componentes de la diversidad a diferentes escalas resulta esencial para entender que factores que explican la diversidad en distintos sistemas (Chase, 2003; Sarr et al., 2005).

La diversidad de plantas espontáneas asociadas a los cultivos puede diferir a lo largo de los agroecosistemas bajo diferentes estilos de agricultura (McLaughlin \& Mineau, 1995; Collins \& Qualset, 1999; de la Fuente et al., 1999; Suárez et al., 2001). La tendencia encontrada es que la diversidad es mayor en sistemas orgánicos que en los convencionales (Krebs et al., 1999; van Elsen, 2000; Hyvonen et al., 2003; Roschewitz et al. 2005; Gabriel et al., 2010; Edesi et al., 2012; Henckel et al., 2015). Esta forma de manejo contribuye a una mayor riqueza de especies nativas, perennes, dicotiledóneas y exclusivas (van Elsen, 2000; Stupino et al., 2008; Travlos et al., 2018). Una de las explicaciones es que los beneficios de la forma de producción orgánica estarían dados por una mayor cantidad de hábitats cultivados y no cultivados (parches) respecto de los convencionales. Algunos autores plantean que la heterogeneidad de hábitat puede ser más importante que la ausencia de insumos químicos (Krebs et al. 1999; Benton et al., 2003; Hole et. al, 2005). La mayor variedad de cultivos genera una mayor diversidad estructural y de hábitats, que podría promover el recambio de especies entre cultivos $(\beta)$ generando una mayor diversidad de espontáneas a nivel de finca ( $\gamma$ ) (Palmer \& Maurer, 1997; Gabriel et al., 2006).

Diversidad Vegetal Espontánea en Agroecosistemas Hortícolas de La Plata y su relación con diferentes estilos de Agricultura: Importancia 
Algunos estudios, realizados en otras regiones, han evaluado la relación entre la riqueza local de especies espontáneas, el manejo y el contexto del paisaje (Balezentiene, 2011; Petit et al., 2013; Henckel et al., 2015; Petit et al., 2015). Algunos encontraron un efecto menor del contexto del paisaje en la composición y riqueza de especies (Weibul et al. 2003; Krauss et al., 2004). Roschewitz et al. (2005) en cambio, encontraron que, tanto el manejo como la complejidad del paisaje (superficie de tierra cultivada), tienen influencia en la diversidad $\beta$ y $\gamma$ de espontáneas (Winqvist et al., 2012; Gabriel et al., 2013). La diversidad puede ser mayor en los sistemas orgánicos que en los convencionales y especialmente en paisajes simples ( $<30 \%$ de habitats seminaturales) con una alta proporción de áreas cultivadas (Tscharntke et al., 2005; Bátary et al., 2010). Roschewitz et al. (2005) encontraron que en paisajes complejos (>60\% de hábitats seminaturales) ambos sistemas alcanzaron un nivel de diversidad similar, por lo que se concluye que el efecto de la complejidad del paisaje sobre la diversidad es mayor en los sistemas convencionales. Por lo tanto, si la heterogeneidad de hábitats dentro de las fincas favorece la presencia de especies, el efecto de la complejidad del paisaje debería ser mayor en sistemas convencionales constituidos por grandes parcelas con pocas especies cultivadas (baja heterogeneidad de hábitats) y un alto uso de insumos. Esto se debe a que la eliminación de especies y la menor capacidad de regeneración del banco de semillas en estos sistemas simplificados y con prácticas intensivas, requieren de un aporte de propágulos provenientes de hábitats naturales del paisaje que compense la disminución de la diversidad dentro de la finca (Roschewitz et al., 2005).

Los estudios vinculados al paisaje se han realizado en zonas templadas donde las áreas de cultivo son grandes y contienen baja diversidad cultivada (Por ejemplo, Fahrig et al., 2015). Sin embargo, es necesario analizar sitios de menor superficie y donde la diversidad cultivada puede ser mayor, como es el caso del Cinturón Hortícola Platense.

En el Capítulo 5, se analizó el efecto del manejo sobre la diversidad a nivel de parcela de cultivo $(\alpha)$. El objetivo de este capítulo es evaluar la relación entre la heterogeneidad de hábitats y la diversidad de plantas espontáneas. Para ello se analiza la diversidad entre cultivos diferentes, lo que representa la escala $\beta$ y, la escala $\gamma$ de diversidad, que refiere el área que reúne todas las parcelas bajo cultivo. Asimismo el capítulo se orienta a caracterizar el paisaje que rodea a cada finca y evaluar si existe alguna contribución diferencial de los

Diversidad Vegetal Espontánea en Agroecosistemas Hortícolas de La Plata y su relación con diferentes estilos de Agricultura: Importancia para la sustentabilidad 


\section{Capítulo 6}

distintos paisajes a la diversidad $\gamma$ de especies acompañantes de los cultivos de los distintos sistemas hortícolas. Para ello se pone a prueba las siguientes hipótesis:

Hipótesis 1:

La heterogeneidad de hábitats en las fincas favorece el aumento de la diversidad Beta y Gamma

- El aumento del número de especies de cultivo y parcelas se corresponde con un aumento de la diversidad Beta (entre cultivos) y Gamma (riqueza total del área cultivada) de especies espontáneas.

- Esta mayor diversidad se traduce en un mayor número de especies exclusivas, un mayor número de especies nativas, perennes y dicotiledóneas. Esta diversidad es máxima en los cultivos orgánicos y disminuye hacia los cultivos convencionales intensivos.

Hipótesis 2:

La complejidad del paisaje influencia la diversidad Gamma a escala de finca y su efecto varía según las particularidades de manejo.

- Existe correlación positiva entre el aumento de la complejidad del paisaje y la diversidad de especies espontáneas asociadas a los cultivos. Este efecto es mayor en las fincas convencionales de baja heterogeneidad de hábitats (pocos cultivos y parcelas).

\subsection{METODOLOGÍA}

\subsubsection{Relevamiento y cálculo de la diversidad Beta y Gamma a escala de finca}

La selección de las fincas y el relevamiento de la vegetación fueron detallados en los Capítulos 3 y 5 . Como se indicó, en cada finca se realizó un muestreo en las parcelas cultivadas, definidas por la presencia de uno o más cultivos dominantes. Se relevó a razón una parcela de cada cultivo diferente, incrementando el número en una unidad de muestreo en el caso que el cultivo supere una hectárea de superficie. Para ello se utilizó un área de $25 \mathrm{~m}^{2}$. Se cuantificó el número de parcelas cultivadas y parcelas no cultivadas, estas últimas

Diversidad Vegetal Espontánea en Agroecosistemas Hortícolas de La Plata y su relación con diferentes estilos de Agricultura: Importancia 
dominadas por vegetación espontánea, pudiendo contener o no residuos de cultivos en post-cosecha.

Se calculó la diversidad $\gamma$ y $\beta$ de especies espontáneas. La diversidad $\gamma$ corresponde a la riqueza total de especies espontáneas encontradas en el sistema (área cultivada). La diversidad $\beta$, es decir la diversidad entre hábitats diferentes, se estimó mediante el índice multiplicativo de Whittaker (1960). La fórmula utilizada es $(\gamma / \alpha)-1$, donde en este caso, $\gamma$ es el número total de especies registradas en el conjunto de parcelas cultivadas y a es el número promedio de especies por parcela de cultivo (Magurran, 1988). La diversidad $\beta$ representa cuánto se diferencian las parcelas de cultivo entre sí en la composición florística. El valor de dicho índice será mínimo cuando la composición sea idéntica y máximo cuando no haya especies en común.

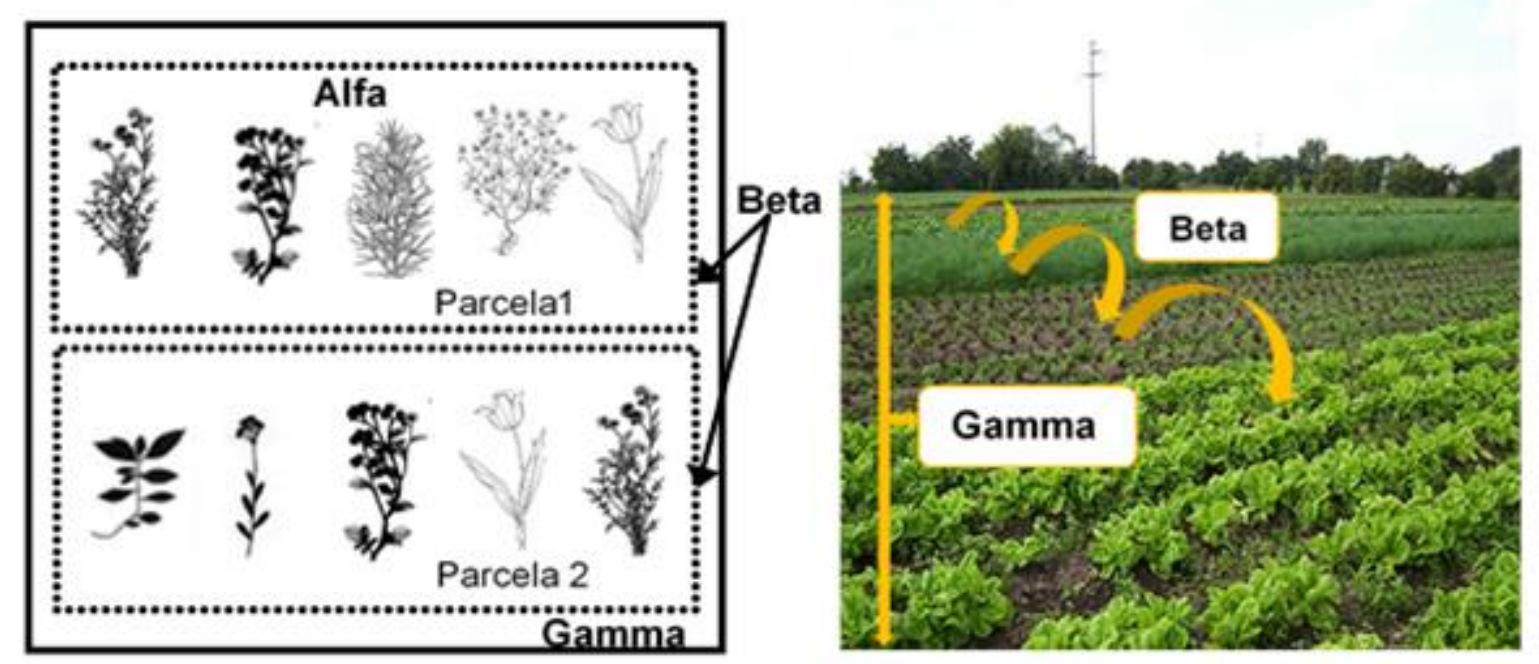

Figura 6.1. Esquema simplificado de las escalas de diversidad de plantas espontáneas en un agroecosistema. En la figura de la izquierda cada recuadro menor punteado define la escala de análisis de diversidad $\alpha$, es decir, diversidad dentro de una parcela de cultivo (Parcela 1 y Parcela 2, respectivamente). La diversidad $\beta$ resulta de comparar parcelas de cultivo entre sí. La línea lisa es el área que comprende la escala de diversidad $\gamma \circ$ diversidad total del agroecosistema. A la derecha se simbolizan el nivel de observación de las escalas $\beta$ y $\gamma$ en las parcelas de un sistema real. Modificado de Stupino et al. (2014), a partir de esquema original de Ferriol Molina \& Merle Farinós (2012).

A partir de la clasificación funcional realizada previamente en el Capítulo 3, se cuantificaron las especies exclusivas de cada tipo de manejo, y el porcentaje de especies

Diversidad Vegetal Espontánea en Agroecosistemas Hortícolas de La Plata y su relación con diferentes estilos de Agricultura: Importancia para la sustentabilidad 
según su ciclo de vida (anual, bienal, perenne), el origen (nativo, exótico, cosmopolita) y la morfología (monocotiledónea y dicotiledónea).

Se realizó consulta bibliográfica de las características funcionales y taxonómicas de los distintos cultivos para establecer que aspectos de los mismos se asocian con una mayor diversidad (Vigliola, 2007; Hurrel et al. 2009; CONABIO, 2006-2012). Se cuantificó en cada finca la diversidad cultivada en cuanto al número de géneros, familias, ciclo de vida (anual, bienal, perenne), distancia de siembra entre hileras (corta, media, larga) y tipo de siembra (directa o almácigo o trasplante, o reproducción prolongada).

\subsubsection{Análisis de correlación entre la diversidad vegetal y las variables que describen la heterogeneidad de las fincas}

Se realizó una correlación entre las principales variables que definen la heterogeneidad dentro de las fincas bajo diferente manejo y la diversidad $\gamma$ y $\beta$ de especies espontáneas. Para ello se utilizaron variables como la riqueza de cultivos, parcelas cultivadas, no cultivadas y totales, tanto en la superficie total como a las correspondientes a cultivo de campo y de invernáculo, por separado. Las variedades de cultivo no fueron consideradas ya que no se pudo obtener la totalidad de nombres científicos y discriminar en el conteo de variedades a campo e invernáculo cuantas eran comunes a ambas modalidades de cultivo.

Se utilizó el coeficiente de correlación de Spearman con el programa Statistica 7.0. El índice oscila entre $-1 \mathrm{y}+1$, indicando asociaciones negativas o positivas respectivamente. El valor numérico indica la magnitud de la correlación. Así, un valor cercano a 1 significa una buena correlación y cercano a 0 una correlación mínima o nula entre dos variables analizadas.

Para la segunda predicción se utilizó el mismo método de correlación entre el manejo y el número de especies exclusivas y, entre el manejo y la abundancia de especies clasificadas según el ciclo de vida, el origen y la morfología. Esto último se analizó en el área de campo e invernáculo por separado. Se realizó un test de Chi-cuadrado $\left(\chi^{2}\right)$ para establecer si existen diferencias entre los distintos tipos de manejo en la proporción (\%) de las características de las especies descriptas en cada una de las categorías funcionales ( $p$ $<0.05)$.

Diversidad Vegetal Espontánea en Agroecosistemas Hortícolas de La Plata y su relación con diferentes estilos de Agricultura: Importancia 


\section{Capítulo 6}

\subsubsection{Localización de las fincas en el contexto del paisaje}

Sobre un mosaico de imágenes satelitales (Google Earth, 2007) se delimitaron y etiquetaron las áreas de estudio previamente seleccionadas. Para ello se ubicaron las fincas en planos de la ciudad y luego se localizaron en la imagen por medio de puntos de referencia (arroyos, cercanía a otro productor conocido, edificaciones), recorriendo la imagen minuciosamente. En algunos casos, se realizaron recorridas en el campo para corroborar la localización de las fincas en la imagen o para encontrar nuevos puntos de referencia que permitieran ubicarlas.

\subsubsection{Definición del área de muestreo a nivel de paisaje}

Tomando como centro cada área de estudio (fincas) se estableció como unidad de muestreo un cuadrado de $1 \mathrm{~km}^{2}$ de superficie que representa el entorno paisajístico que actúa como fuente de propágulos (Roschewitz et al. 2005). Para representar la unidad de muestreo, en cada finca se ubicó un punto central de referencia y dos pares de puntos que son los extremos de un sistema de coordenadas cartesianas con origen en dicho punto central (Figura 6.2). Cada eje definido por un par de puntos tuvo $1 \mathrm{~km}$ de longitud, de manera que el área comprendida entre los cuatro puntos representa $1 \mathrm{~km}^{2}$. Esta tarea debió hacerse por limitaciones del programa, ya que no permitió definir áreas directamente. Se exportó cada imagen de las distintas fincas al programa Corel Draw, donde se superpuso una grilla de $10 \times 10$ celdas (100 puntos) de 20 × $20 \mathrm{~cm}$. Se ajustó la imagen de 1 km² a la grilla en función de los puntos de referencia verificando que no hubiese distorsión de la misma.

Diversidad Vegetal Espontánea en Agroecosistemas Hortícolas de La Plata y su relación con diferentes estilos de Agricultura: Importancia 


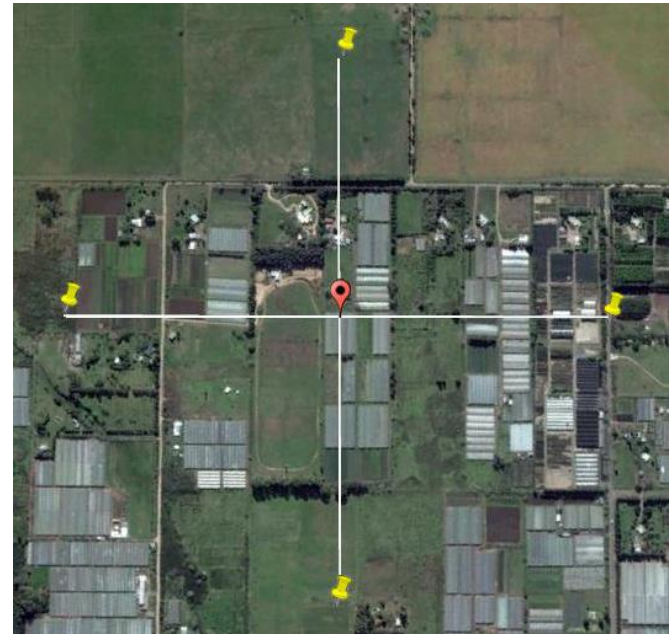

a)

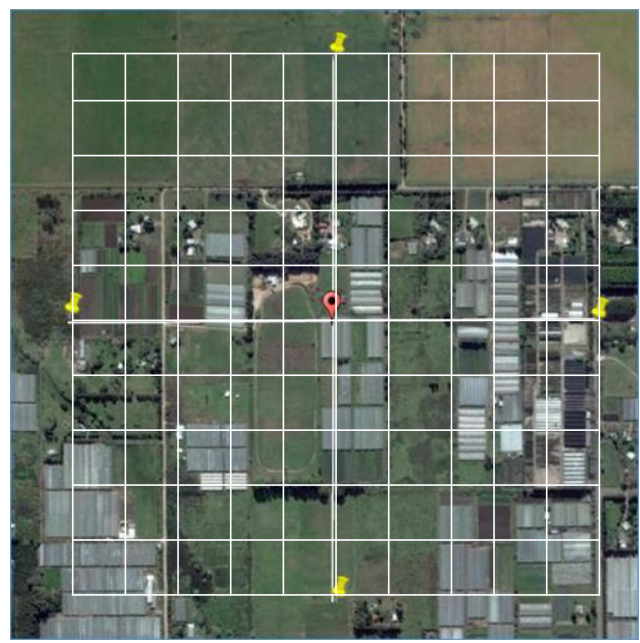

b)

Figura 6.2. Esquema de los pasos realizados para localizar las fincas y delimitar el área y el método de muestreo del paisaje: a) Localización de las fincas y puntos de referencia; b) superposición a escala de una grilla de 100 puntos (10 celdas x 10 celdas de $20 \mathrm{~cm}$ ) que representa la unidad de muestreo.

\subsubsection{Clasificación y cuantificación de unidades del paisaje}

A partir de la observación realizada a campo y en el mosaico de imágenes satelitales, se establecieron criterios de uso de la tierra y fisonómico para la clasificación de unidades de paisaje. En base a estos criterios se definieron las unidades: 1) monte forestal: caracterizada por el dominio de árboles (Figura 6.3), 2) peridoméstico: sector de árboles y/o jardín que rodean la casa dentro de un radio inferior a $20 \mathrm{~m} \mathrm{3}$ ) área parquizada: sector dominado por herbáceas de corte periódico (ej. casas quintas) 4) ganadería: área con predominio de herbáceas espontáneas sometidas a pastoreo 5) área cultivada a cielo abierto: con presencia de parcelas o lotes diferentes etapas de cultivo (descanso, rastrojo, cultivo) 6) área cultivada bajo cubierta: caracterizada por el cultivo en invernáculo 7) campo sucesional herbáceo: campos sin uso actual con presencia de estrato herbáceo 8) campo sucesional leñoso: campos sin uso actual con presencia de herbáceas y leñosas 9) corredor natural: elemento linear del paisaje caracterizado por la presencia de vegetación próxima a un camino no pavimentado 10) calle asfaltada 11) construcciones edilicias 12) forrajera: unidad dominada por pasturas artificiales. 


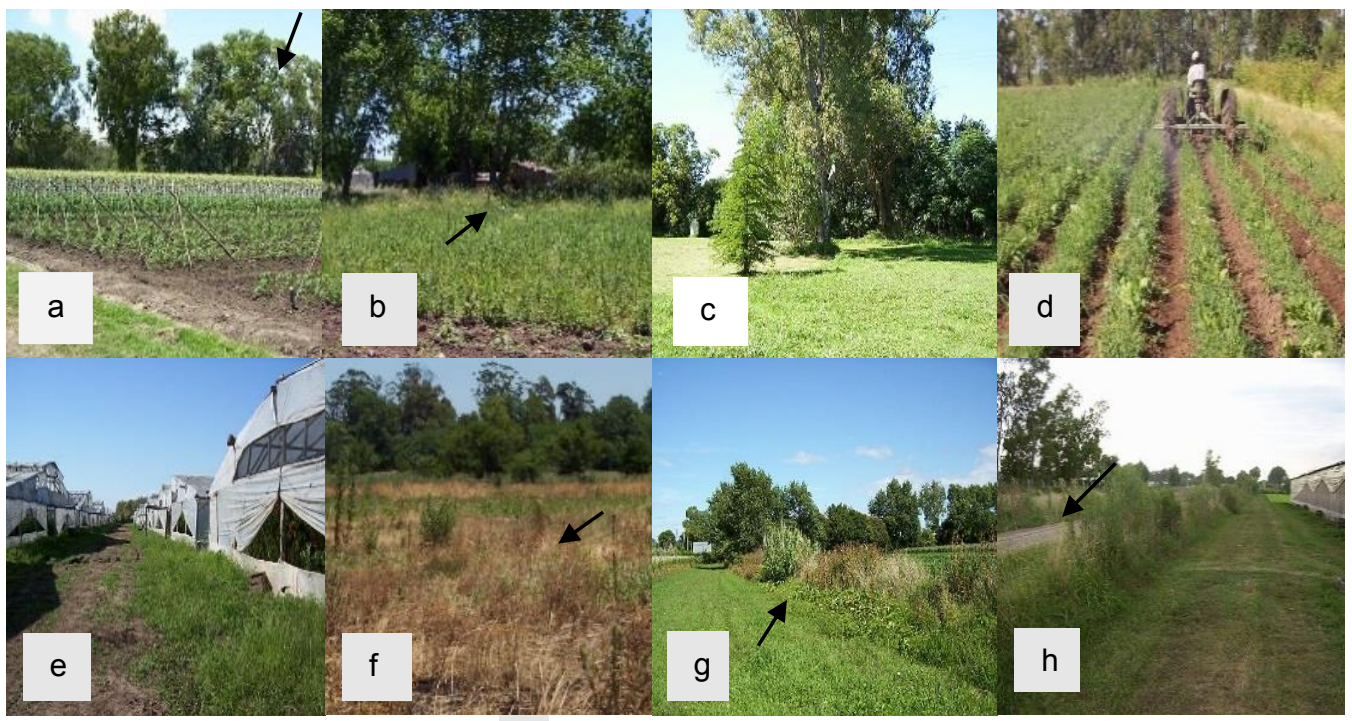

Figura 6.3. Ejemplos de unidades del paisaje: a) monte forestal; b) peridoméstico; c) área parquizada; d) área cultivada a cielo abierto; e) área cultivada bajo cubierta; f) campo sucesional herbáceo; $g$ y h) corredor natural. Las flechas negras indican la unidad correspondiente. Fotografías de la autora.

En una planilla impresa se registraron todas las unidades que se encuentran en cada punto de intersección (en un total de 100 puntos). El relevamiento comprendió 32 entornos de las fincas estudiadas, representando un total de 3200 unidades identificadas. Se corroboraron en el campo las unidades que no tenían una buena apreciación en la imagen. Esto implicó un total de 21 recorridas en los alrededores fincas. Para precisar los caminos a recorrer en el campo y la localización de las unidades se utilizaron planos esquemáticos y fotografías de las grillas construidas.

\subsubsection{Cálculo de índices de diversidad del paisaje}

Se calculó el índice de diversidad de Shannon como medida de la heterogeneidad del entorno, cuya fórmula es $\mathrm{H}=-\Sigma \mathrm{pi}^{*}$ In pi, donde pi es la abundancia proporcional la unidad de paisaje i (Magurran, 1988). Para ello se utilizó el programa MVSP 3.1. Se calculó, además, la riqueza de tipos de unidades del paisaje, el porcentaje de área cultivada y de áreas seminaturales. Las unidades cultivadas fueron área cultivada a cielo abierto (campo), área cultivada bajo cubierta (invernáculo) y forrajera. Dentro de la categoría seminatural se consideraron las unidades corredor natural, monte, campo sucesional herbáceo, campo sucesional leñoso y ganadería.

Diversidad Vegetal Espontánea en Agroecosistemas Hortícolas de La Plata y su relación con diferentes estilos de Agricultura: Importancia 


\subsubsection{Análisis de correlación entre la diversidad vegetal de las fincas y las variables que describen la heterogeneidad del paisaje}

Al igual que para las fincas, se analizó la correlación entre la diversidad $\gamma$ y $\beta$ del total de cultivos de cada finca y las diversidad de paisaje. Se calculó el coeficiente de correlación de Spearman con el programa Statistica 7.0. Como variables que definen la heterogeneidad del paisaje se utilizó el índice Shannon del paisaje, la proporción de áreas seminaturales y cultivadas. Se exploró, a su vez, el efecto de cada unidad de paisaje por separado.

\subsection{RESULTADOS}

\subsubsection{Relación entre la diversidad Beta y Gamma y la heterogeneidad de hábitats}

La diversidad $\gamma$ de especies espontáneas promedio total fue superior en las fincas orgánicas, que en las convencionales, que no tuvieron diferencias entre sí (Tabla 6.1). En ambos sistemas de cultivo, a campo y en invernáculo, la diversidad fue mayor en el manejo orgánico que en los otros dos. La diversidad $\beta$ también fue mayor en las fincas orgánicas que en las convencionales intensivas tanto en la superficie total como a campo. En general, el invernáculo presentó menor número de especies y, un menor recambio entre cultivos $(\beta)$ que en la superficie a campo (con excepción del manejo convencional intensivo). Los manejos de bajos insumos presentaron valores intermedios en los diferentes análisis.

Tabla 6.1. Valores promedios de diversidad $\gamma$ y $\beta$ de especies espontáneas en la superficie cultivada total, sólo a campo e invernáculo respectivamente en, fincas orgánicas (ORG), convencionales de bajos insumos $(\mathrm{BI})$ y convencionales intensivas $(\mathrm{Cl})$.

\begin{tabular}{lcccccc}
\hline Manejo & \multicolumn{3}{c}{ Diversidad Gamma } & \multicolumn{3}{c}{ Diversidad Beta } \\
\hline & $\begin{array}{c}\text { Total } \\
\text { promedio(rango) }\end{array}$ & Campo & Invernáculo & Total & Campo & Invernáculo \\
& $47(64-33)$ & 44 & 24 & 3.5 & 3.1 & \\
\hline ORG & $31(42-21)$ & 30 & 6 & 2.7 & 2.6 & 1 \\
BI & $29(50-13)$ & 24 & 19 & 3.1 & 2.3 & 2.9 \\
Cl & 29 & & & & & \\
\hline
\end{tabular}

Se encontró una correlación negativa significativa entre la diversidad $\gamma$ total y el tipo de manejo (Tabla 6.2), lo que indica que la riqueza total de especies disminuyó a medida que

Diversidad Vegetal Espontánea en Agroecosistemas Hortícolas de La Plata y su relación con diferentes estilos de Agricultura: Importancia 
se incrementa la intensidad de manejo (es decir, la diversidad $\gamma$ es menor en los sistemas convencionales intensivos). Además, se encontró una asociación positiva con el número de especies cultivadas, parcelas cultivadas y totales (cultivadas y no cultivadas). La diversidad $\gamma$ a campo mostró la misma tendencia. Esto significa que la diversidad de espontáneas decrece cuando incrementa la intensidad de manejo y aumenta con las variables que representan una mayor heterogeneidad. El número de especies cultivadas y totales fueron las variables donde se encontró la mejor correlación.

Tabla 6.2. Valores de correlación entre la diversidad $\gamma$ y $\beta$ de plantas espontáneas y el tipo de manejo (orgánico, convencional de bajos insumos y convencional intensivo) incluyendo la modalidad a campo e invernáculo juntas. El asterisco $\left(^{*}\right)$ indica los valores significativos, $(p \leq 0.05)$.

\begin{tabular}{ccccccc}
\hline Variable & \multicolumn{3}{c}{ Gamma } & \multicolumn{3}{c}{ Beta } \\
& Total & Campo & Invernáculo & Total & Campo & Invernáculo \\
\hline Tipo de manejo & $-0,45^{*}$ & $-0,60^{*}$ & $-0,09$ & $-0,04$ & $-0,44^{*}$ & $0,52^{*}$ \\
Especies cultivadas & $0,66^{*}$ & $0,66^{*}$ & 0,22 & $0,40^{*}$ & $0,58^{*}$ & $-0,38$ \\
Parcelas cultivadas & $0,60^{*}$ & $0,58^{*}$ & 0,29 & 0,33 & $0,64^{*}$ & 0,09 \\
Parcelas no cultivadas & $0,39^{*}$ & $0,54^{*}$ & 0,05 & 0,15 & $0,52^{*}$ & $-0,19$ \\
Parcelas totales & $0,74^{*}$ & $0,59^{*}$ & 0,26 & $0,43^{*}$ & $0,62^{*}$ & $-0,09$ \\
\hline
\end{tabular}

La diversidad $\beta$ total se relacionó con la riqueza de parcelas cultivadas y totales. En cambio, considerando solo el manejo a campo, mostró la misma tendencia que $\gamma$.

A diferencia de lo observado a campo, en el invernáculo se encontró que únicamente la diversidad $\beta$ estuvo asociada, de manera positiva con el tipo de manejo, lo que significa que aumentó en dirección del incremento de la intensidad de manejo siendo mayor, por ende, en los cultivos convencionales intensivos.

Se encontró que la diversidad $\gamma$ se asoció, además, de manera significativa con distintas variables que describen características funcionales de los cultivos. Las de mayor valor de correlación relacionadas con la riqueza de especies cultivadas fueron: géneros $(r=0,64 ; p \leq 0,05)$, familias $(r=0,49 ; p \leq 0,05)$, anuales $(r=0,62 ; p \leq 0,05)$, bienales $(r=0,57$; $p \leq 0,05)$, siembra directa $(r=0,61 ; p \leq 0,05)$ y por trasplante $(r=0,59 ; p \leq 0,05)$. La diversidad 


\section{Capítulo 6}

$\beta$, tuvo bajos niveles de correlación principalmente con los cultivos anuales $(r=0,43 ; p \leq$ $0,05)$ y la siembra por trasplante $(r=0,50 ; p \leq 0,05)$.

La riqueza de especies exclusivas o raras se asoció de manera negativa y significativa con el incremento de la intensidad de manejo $(r=-0,50 ; p>0.5)$. Esto significa que la presencia de especies exclusivas es mayor en las fincas orgánicas y disminuye hacia las convencionales.

Todas las características de las especies espontáneas que describen el origen, el ciclo de vida y la morfología, tuvieron una asociación negativa significativa solamente con el manejo a campo (siendo no significativas en invernáculo) (Tabla 6.3), lo que significa que disminuyen en su abundancia con el incremento de la intensidad de manejo.

Tabla 6.3.Valores de correlación entre el tipo de manejo (orgánico, convencional de bajos insumos y convencional intensivo) y la abundancia de plantas espontáneas según las categorías de ciclo de vida, origen y morfología. El asterisco $\left(^{*}\right)$ indica los valores significativos $(p \leq 0.05)$.

\begin{tabular}{ll}
\hline Características & Tipo de manejo \\
\hline Nativas & $-0,50^{*}$ \\
Exóticas & $-0,68^{*}$ \\
Cosmopolitas & $-0,37^{*}$ \\
Anuales & $-0,45^{*}$ \\
Bienales & $-0,70^{*}$ \\
Perennes & $-0,63^{*}$ \\
Dicotiledóneas & $-0,61^{*}$ \\
Monocotiledóneas & $-0.53^{*}$ \\
\hline
\end{tabular}

El análisis conjunto de datos de manejo a campo e invernáculo de las fincas (nivel $\gamma$ ) mostró el predominio de plantas exóticas, anuales y dicotiledóneas en todos los tipos de manejo (Figura 6.4). Asimismo, en los manejos orgánicos la mayor riqueza hace más notoria la presencia de especies nativas, perennes y dicotiledóneas. El test de Chi-cuadrado no mostró diferencias significativas entre manejos en los porcentajes de las características de las plantas agrupadas según el ciclo de vida $\left(\chi^{2}=1,00 ; p=0,909\right)$, el origen $\left(\chi^{2}=3,35\right.$; $p=0,501)$ y la morfología $\left(\chi^{2}=0,08 ; p=0,961\right)$, respectivamente. 


\section{Capítulo 6}

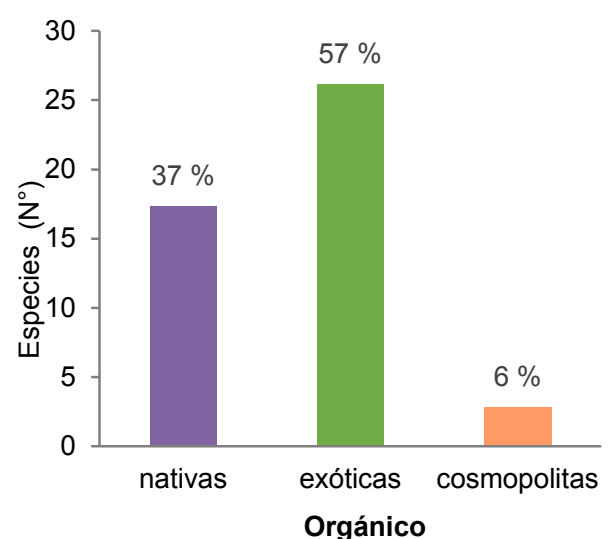

a)

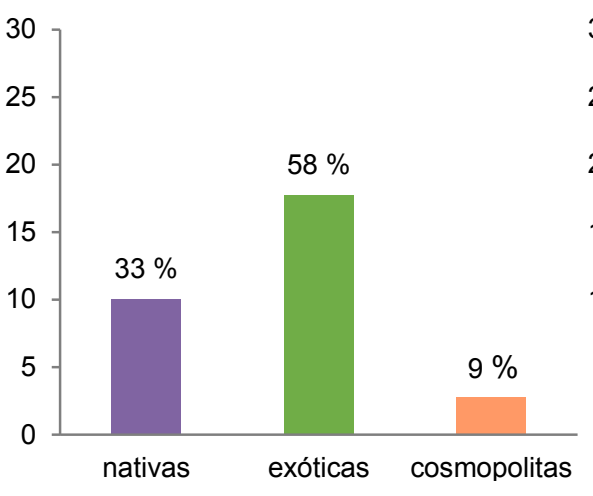

Convencional Bajos Insumos

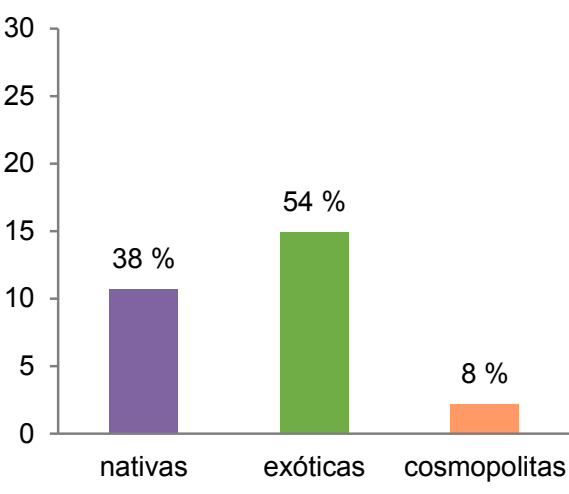

Convencional Intensivo
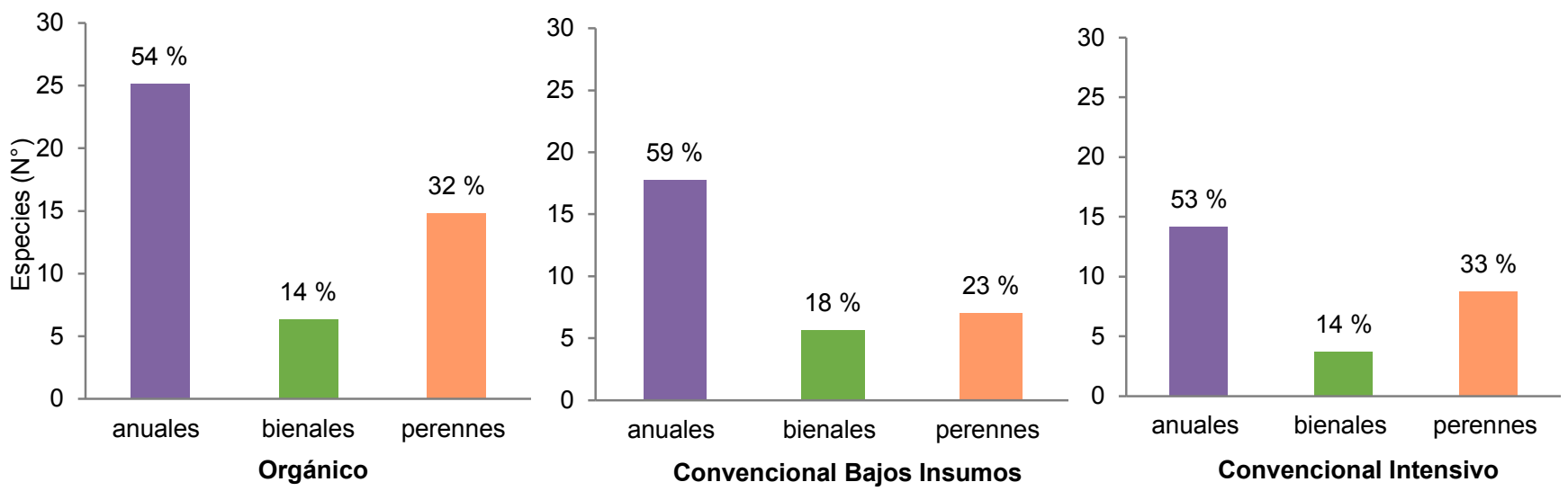

b)
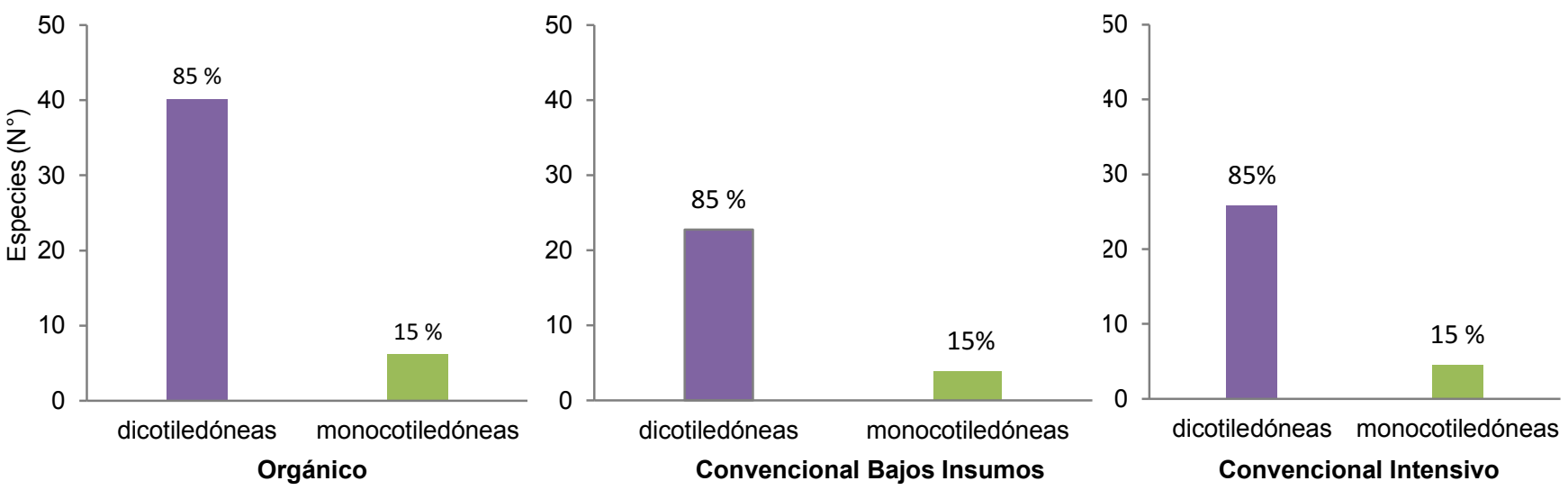

c)

Figura 6.4. Riqueza de especies de plantas espontáneas expresadas como número $\left(\mathrm{N}^{\circ}\right.$, eje $\left.\mathrm{Y}\right)$ y porcentaje (\%, sobre cada columna) clasificadas según el origen (a), el ciclo de vida (b) y la morfología (c) para cada tipo de manejo: Orgánico, Convencional de Bajos Insumos y Convencional intensivo. 


\subsubsection{Caracterización del entorno del paisaje que rodea las fincas}

Se encontraron 12 unidades del paisaje. Las unidades seminaturales correspondieron a corredor natural, monte, campo sucesional leñoso, campo sucesional herbáceo, ganadería y peridoméstico. Las unidades cultivadas fueron clasificadas en área cultivada a cielo abierto (campo), área cultivada bajo cubierta (invernáculo) y área forrajera. Además, se encontraron áreas parquizadas, calles asfaltadas y construcciones edilicias. Las unidades seminaturales en conjunto representaron en promedio el $46 \%$ del total del paisaje relevado ( \pm 18 . Min: 13 , máx.: 77$)$ y las cultivadas el $43 \%$ ( \pm 19 . Min: 13, máx.: 86).

Las unidades de paisaje de ambientes seminaturales más abundantes fueron el campo sucesional herbáceo y el área cultivada a cielo abierto para todos los entornos de las fincas bajo diferente manejo (Figura 6.5). Las fincas de bajos insumos y convencionales intensivas tuvieron una mayor proporción de áreas cultivadas a campo. El área bajo cubierta fue similar para las fincas orgánicas e intensivas. El resto de las unidades espaciales tuvieron valores bajos de cobertura (alrededor del $10 \%$ o menos).

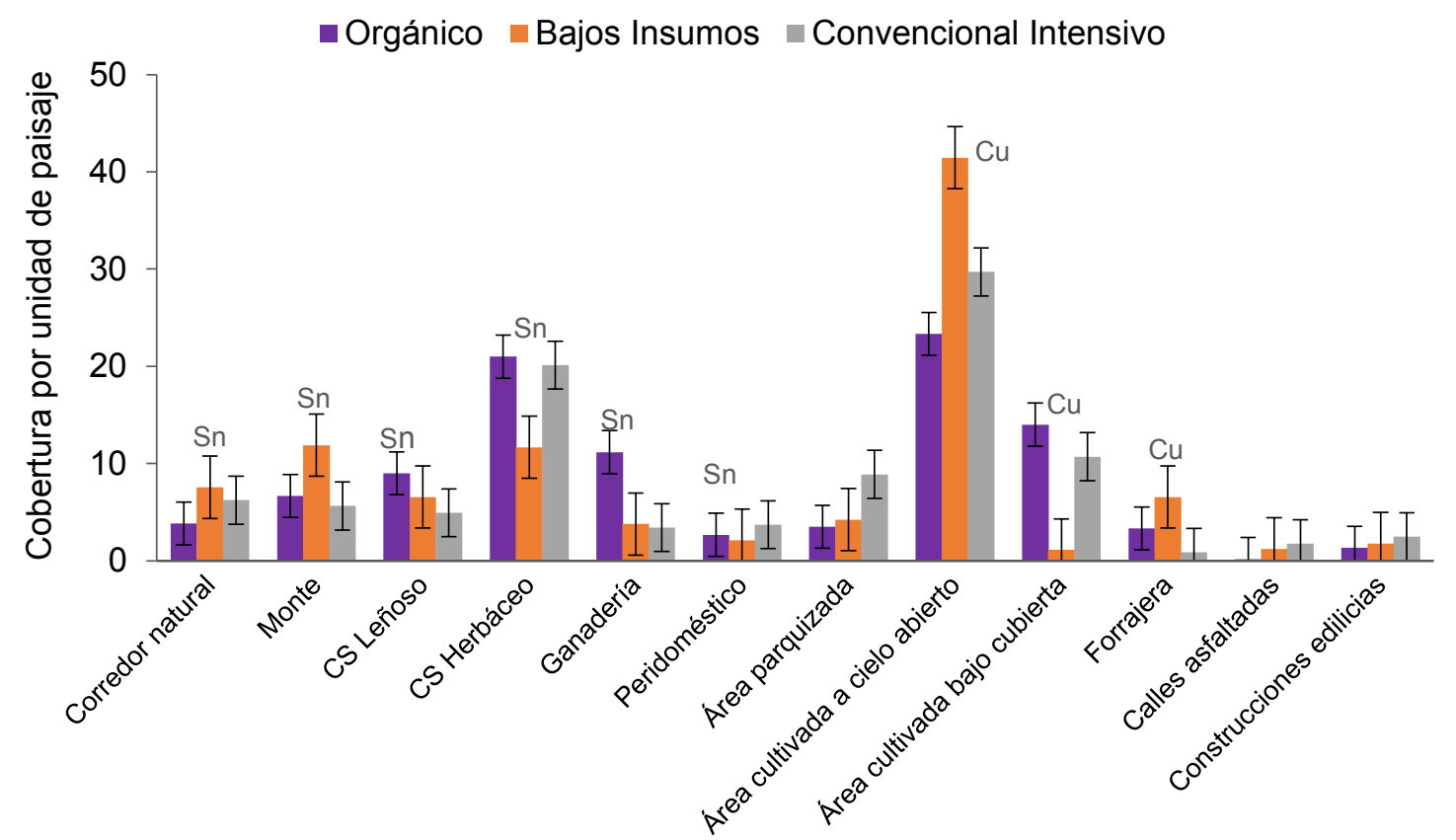

Figura 6.5. Porcentaje de cobertura de las principales unidades del paisaje seminaturales y cultivadas para cada tipo de manejo: orgánico, convencional de bajos insumos y convencional intensivo. Las barras representan el error estándar a 95\% de significancia. CS: campo sucesional. Las abreviaciones en la parte superior indican si la unidad es seminatural ( $\mathrm{Sn}$ ) o cultivada ( $\mathrm{Cu}$ ).

Diversidad Vegetal Espontánea en Agroecosistemas Hortícolas de La Plata y su relación con diferentes estilos de Agricultura: Importancia 
No se encontró correlación significativa entre las diversidades $\gamma$ y $\beta$ dentro del área total cultivada de las fincas y el índice de Shannon del paisaje $(r \gamma=-0,16, r \beta=-0,02 ; p \leq 0,05)$, ni con la riqueza de unidades $(r \gamma=-0,33, r \beta=-0,003 ; p \leq 0,05)$, o la proporción de área cultivada $(r \gamma=-0,01, r \beta=0,001 ; p \leq 0,05)$ y seminatural $(r \gamma=-0,03, r \beta=0,01 ; p \leq 0,05)$.

\subsection{DISCUSIÓN}

\subsubsection{Relación entre la heterogeneidad y la diversidad Beta y Gamma}

La mayor diversidad $\gamma$ encontrada en las fincas orgánicas coincide con numerosos estudios realizados en otras regiones (Krebs et al., 1999; van Elsen, 2000; Hyvonen et al., 2003; Roschewitz et al. 2005; Gabriel et al., 2010; Edesi et al., 2012; Henckel et al., 2015; Gabriel et al., 2016). Los resultados confirman la hipótesis que la heterogeneidad de hábitats favorece el incremento de la diversidad Gama. La mayor diversidad se asoció al mayor número de cultivos, parcelas totales y cultivadas considerando tanto la superficie total de la finca como el área bajo manejo a campo. Por otro lado, en este análisis, $\gamma$ se asoció negativamente con el tipo de manejo. Estos resultados coinciden con lo observado en el Capítulo 4 donde la mayor diversidad cultivada caracterizó a los estilos de agricultura orgánicos disminuyendo hacia los sistemas convencionales de mayor intensidad de manejo, ambos establecidos en un gradiente.

La diversidad $\beta$ en las fincas orgánicas fue superior a la encontrada en las convencionales tanto en la superficie total como a campo y se relacionó con las mismas variables que establecen la heterogeneidad con $\gamma$, aunque $\beta$ total se asoció sólo con la riqueza de cultivos y de parcelas totales (cultivadas y no cultivadas). Estas diferencias podrían deberse a la incorporación del invernáculo en el análisis de la superficie total, ya que se caracteriza por el cultivo continuo de unas pocas especies cultivadas y parcelas, lo que podría minimizar el efecto sobre los valores de correlación. Esto se corresponde, además con que en el invernáculo, $\beta$ no se relacionó con ninguna de las variables que describen la heterogeneidad.

Por otro lado, la diversidad $\beta$ en el invernáculo se asoció de manera positiva con el tipo de manejo. Esto sugiere que el manejo más intensivo presenta un mayor recambio de

Diversidad Vegetal Espontánea en Agroecosistemas Hortícolas de La Plata y su relación con diferentes estilos de Agricultura: Importancia 
especies que en los manejos orgánicos. Esto podría corresponderse por un lado, a que el invernáculo representa la modalidad con mayor nivel de intensificación (Capítulo 4), con alta disponibilidad de recursos y condiciones favorables (fertilizantes, agua, temperaturas más cálidas) que podría favorecer la presencia de especies oportunistas, que escapan o se "recuperan" luego de la aplicación de esterilizantes del suelo. A su vez, la menor riqueza promedio encontrada en los cultivos bajo invernáculo a nivel $\alpha$ podría incrementar el recambio de especies que muestra las diferencias entre parcelas (Capitulo 5).

Varias teorías ecológicas intentan explicar el ensamblaje de comunidades y, en los agroecosistemas en particular, aún resulta difícil establecer los factores que influyen sobre las especies espontáneas (Perronne et al., 2015). No obstante, se han propuesto algunas interpretaciones que podrían explicar la mayor diversidad asociada a la riqueza cultivada. Cada especie de planta afecta diferencialmente el ambiente creando una pequeña heterogeneidad ambiental, que crea oportunidades para que se establezcan nuevas plantas con diferentes preferencias. Cada especie de cultivo utiliza diferentes recursos (temporales y espaciales), presenta variaciones en la microflora de la rizófera y en las condiciones de luz dada por la estructura del canopeo. Esta heterogeneidad microambiental dada por los tipos de cultivos, promueve una riqueza taxonómica de propágulos y una variedad en los efectos sobre la intensidad de la luz captada por las plantas, potenciando una diversidad de plantas emergentes (Palmer \& Mauler, 199; Benech-Arnold et al., 2000; Ghersa \& Martinez Ghersa, 2000). A su vez, la variedad de cultivos previene el desarrollo de unas pocas especies dominantes al presentar diferentes tiempos de siembra, cosecha y ciclo de vida (Liebman \& Dyck, 1993; Mac Laugling \& Mineau, 1995; Poggio et al., 2004). Así, una cierta combinación de especies cultivadas puede promover, respecto de un monocultivo, una mayor presencia de especies asociadas, al incluir especies de cultivo que por sus características generen condiciones favorables para el establecimiento de espontáneas (Palmer \& Mauler, 1997).

Por otro lado, la especie cultivada puede tener un efecto indirecto sobre las plantas asociadas. Por ejemplo, el régimen de fertilización, el manejo del suelo y uso de herbicidas dependen del tipo de cultivo, y estos factores actúan a modo de filtros abióticos determinando la estructura y diversidad de la comunidad de plantas espontáneas (Perronne et al., 2015). Estos aspectos sugieren que no solo es importante la especie cultivada, sino también sus características funcionales y taxonómicas que incrementan la heterogeneidad

Diversidad Vegetal Espontánea en Agroecosistemas Hortícolas de La Plata y su relación con diferentes estilos de Agricultura: Importancia para la sustentabilidad 
microambiental. En este sentido, se encontró que, una mayor riqueza de géneros y familias de cultivos, cultivos anuales y bienales y de siembra directa o trasplante se asociaron de manera positiva con la diversidad $\gamma$ y $\beta$.

La diversidad $\beta$ indica que las parcelas cultivadas difieren en la composición de especies espontáneas y esto podría estar ligado a las diferencias entre los cultivos en sitios donde no se han aplicado herbicidas en años, lo que permite la acumulación de un banco de propágulos (Hole et al., 2005; Romero et al., 2008; Bavec \& Bavec, 2015). En cambio, la homogeneización de los hábitats a través del uso de fertilizantes y herbicidas y un esquema de rotación menos diverso, se asoció con sistemas intensivos que han mostrado disminuir la riqueza de espontáneas, al seleccionar pocas especies dominantes con requerimientos similares al cultivo (Benton, 2003; Poggio et al., 2004; Borgy et al., 2012). Esto coincide con la composición florística más homogénea encontrada a nivel $\alpha$ en cultivos convencionales (Capítulo 5). Bajo estas condiciones similares que se repiten año a año, se propagan malezas consideradas "problema" por su habilidad competitiva y alta densidad (Liebman \& Dyck, 1993). Un ejemplo de esta dominancia es el caso de Cyperus rotundus, una especie de alta tasa reproductiva e invasora que fue más frecuente en las fincas convencionales intensivas de pocas especies cultivadas y fue considerada un problema por los agricultores.

La mayor riqueza de especies exóticas, anuales y dicotiledóneas a nivel $\gamma$ concuerda con la tendencia encontrada a nivel $\alpha$ en el Capítulo 5 , lo que caracteriza a los sistemas que presentan un régimen de disturbio periódico (entendido este como una medida de la fracción de biomasa removida) (Grime, 1977; Suárez et al., 2001; de la Fuente et al, 2006; Poggio et al., 2013). El predominio de especies nativas, perennes y dicotiledóneas en los manejos orgánicos, confirma la segunda predicción y coincide con los resultados de otros trabajos (van Elsen, 2000; Stupino et al., 2008, Travlos et al., 2018). Estas características funcionales encontradas a nivel $\gamma$ concuerdan con los resultados obtenidos en una menor escala de análisis ( $\alpha$ ) en el Capítulo 5 , confirmando que los cultivos orgánicos se asocian a sistemas de menor disturbio respecto de los convencionales (Travlos et al., 2018). A alta diversidad y disturbios intermedios coexisten especies de rápido crecimiento generalistas con especies competitivas dominantes de crecimiento lento y de alta persistencia en el banco de semillas, como las perennes (Armengot et al., 2012). Grime (1977) establece que existe un feedback (retroalimentación) entre la diversidad y el disturbio. La mayor diversidad

Diversidad Vegetal Espontánea en Agroecosistemas Hortícolas de La Plata y su relación con diferentes estilos de Agricultura: Importancia para la sustentabilidad 
puede disminuir el efecto del disturbio y generar resistencia a la invasión de exóticas (Hugues et al., 2007). Esto podría explicar también la presencia de un mayor número de nativas en las fincas orgánicas. En el presente estudio el efecto de los distintos manejos se verifica esencialmente en la riqueza de las especies acompañantes, pero no siempre (con la excepción de los cultivos a campo) se relaciona significativamente con categorías morfofuncionales, y en ningún caso con la estructura relativa (proporcionalidad) de los agrupamientos en las categorías de plantas analizadas.

Un grupo de plantas comunes caracterizaron a los estilos de agricultura orgánicos y convencionales, como se indicó en el Capítulo 5. Sin embargo, como se predijo, las fincas orgánicas se destacaron por la mayor riqueza de especies espontáneas raras o exclusivas a escala $\gamma$. Estos resultados coinciden con lo indicado por numerosos autores (Van Elsen, 2000; Romero et al. 2008, Stupino et al., 2008, Travlos et al., 2018). Crowder et al. (2012), encontraron que en conversiones a la agricultura orgánica se incrementa la abundancia de especies raras por encima de la adición de nuevas especies, manteniendo la uniformidad. Esto indica la importancia del estilo de agricultura en determinar la presencia de especies de baja frecuencia. Varias razones explican la rareza, como la amplitud estrecha del nicho o la restringida habilidad de dispersión (Gaston, 1994). La agricultura orgánica puede favorecer a especies no adaptadas a los sistemas convencionales por ser sensibles a los métodos de control (nicho estrecho). Esto podría relacionarse con la presión de selección que ejercen el desmalezado químico y la fertilización nitrogenada en manejos convencionales, principales variables relacionadas con la diversidad $\alpha$ (Capítulo 5). La aplicación de herbicidas cambia la estructura y composición, reduce la densidad de especies susceptibles e incrementa la presencia de especies comunes tolerantes. Estas últimas pueden estar menos afectadas por las prácticas de manejo y tienden a incrementar su dominancia (Omezine \& Texeira da Silva, 2012). De la misma manera el efecto de los fertilizantes nitrogenados incrementa la biomasa del cultivo limitando el desarrollo de especies tolerantes a condiciones de poca luz. El incremento en el número de especies raras o poco frecuentes puede corresponderse con la mayor tasa de recambio entre parcelas encontrada en los sistemas orgánicos (Alto $\beta$ ).

En síntesis, como fue planteado en la hipótesis, la mayor variedad de cultivos y parcelas genera una mayor diversidad estructural y de hábitats que favorece la presencia de plantas espontáneas a escala $\beta$ y $\gamma$ (Gabriel et al., 2006). Por lo tanto, los beneficios de

Diversidad Vegetal Espontánea en Agroecosistemas Hortícolas de La Plata y su relación con diferentes estilos de Agricultura: Importancia para la sustentabilidad 
la producción orgánica estarían dados, en parte, por una mayor calidad y cantidad de hábitats cultivados y parcelas comparado con los sistemas convencionales. Estos aspectos se superponen con los efectos de las prácticas de manejo analizadas a escala $\alpha$ (Capítulo $4)$.

Uno de los aspectos a tener en cuenta, es que las fincas son diversas en su organización y distribución espacial aún en la misma región y tipo de producción. El diseño establecido determina una mayor o menor heterogeneidad de hábitats. Por lo tanto, el agricultor juega un papel fundamental en determinar el nivel de biodiversidad de su finca, ya que es quien decide qué y cómo cultivar. Al margen de las diferencias encontradas entre manejos, el agricultor convencional podría tener una mayor riqueza de cultivos o eliminar hábitats no cultivados, con lo cual la actitud del agricultor es un factor importante en determinar la diversidad a escala de finca. En la zona de La Plata, dicha heterogeneidad alcanzada es condicionada además, por la forma de comercialización. Los agricultores orgánicos o de base agroecológica que comercializan sus productos a través de canales cortos de venta como las ferias o el reparto a domicilio, requieren tener un mayor número de productos para satisfacer la demanda, proceso mediante el cual el consumidor potencia ,en su retroalimentación con el agricultor hortícola, un efecto indirecto sobre la biodiversidad (Capítulo 4). Este diseño más diverso se contrapone con los agricultores convencionales, que venden sus productos en mercados concentradores que requieren grandes volúmenes de un mismo producto.

\subsubsection{Relación entre la diversidad y el entorno del paisaje}

Algunos autores encontraron que tanto el manejo como la complejidad del paisaje (superficie de tierra cultivada) tienen influencia en la diversidad $\gamma$ de espontáneas (Roschewitz et al. 2005; Bátary et al., 2011; Winqvist et al., 2012), siendo la diversidad mayor en los sistemas orgánicos que en los convencionales y especialmente en paisajes simples con una alta proporción de áreas cultivadas (Bátary et al., 2011). En esta tesis, las diversidades $\gamma$ y $\beta$ encontradas dentro de las fincas no se relacionaron con las métricas analizadas del entorno del paisaje. Esto coincide con otros autores que encontraron un efecto menor del paisaje en la composición y riqueza de especies a escala de finca (Weibul \& Östman, 2003; Krauss et al., 2004; Armengot, 2011). El tipo de hábitats es el factor más importante que explica la variación a través de los sitios locales en el paisaje agrícola y la

Diversidad Vegetal Espontánea en Agroecosistemas Hortícolas de La Plata y su relación con diferentes estilos de Agricultura: Importancia 
calidad del mismo está determinado por factores antrópicos de uso de la tierra y manejo (Baessler \& Klotz, 2006). Weibul \& Östman (2003) encontraron que el efecto del paisaje puede variar entre los diferentes tipos de hábitats, siendo mayor en los hábitats manejados intensamente que en hábitats más estables. A su vez, plantean que en paisajes homogéneos, la distancia del sitio disturbado al refugio potencial es mayor, disminuyendo la posibilidad de recolonización. Por lo tanto, el efecto de la complejidad del paisaje sobre la composición de especies podría ser más importante para organismos de mayor movilidad que para las plantas.

En este análisis, la similar riqueza, cobertura y tipo de unidades de paisaje encontrada entre las fincas orgánicas y convencionales sugieren que se encuentran en entornos parecidos de complejidad intermedia (tanto las unidades sumadas de áreas seminaturales como las cultivadas, representaron aproximadamente un $45 \%$ de la cobertura del paisaje). Las diferencias entre manejos fueron menores, siendo algo mayor la proporción de ambientes seminaturales en los entornos de fincas orgánicas e intensivas, y de áreas cultivadas en las de bajos insumos. No se destacaron paisajes simples, con alta proporción de área cultivada. La complejidad similar del paisaje y en nivel intermedios, no permite aceptar la hipótesis y predicción asociada, que establece la posible correlación positiva entre el aumento de la complejidad del paisaje y la diversidad de especies espontáneas asociadas a los cultivos, y el mayor efecto en los manejos convencionales. Los entornos parecidos pueden deberse a que las fincas orgánicas no se encuentran aun particularmente en una región como ocurre en otros países donde se definen áreas bajo esquemas agroambientales con incentivos a agricultores que produzcan de forma amigable con el ambiente (Gabriel et al., 2006; Schneider et al., 2014). En cambio, las fincas orgánicas de la zona están inmersas en un paisaje diverso con predominio de áreas cultivadas convencionales.

Las diversidades $\gamma$ y $\beta$ no se relacionaron con el nivel de complejidad del paisaje, pero sí con el tipo de manejo. Esto sugiere que, en el Cinturón Hortícola de La Plata, el efecto del manejo tiene todavía mayor influencia sobre la diversidad local de las fincas que la complejidad del paisaje. A nivel de finca, esto podría deberse a que los entornos parecidos en la composición de hábitats no productivos pueden generar un efecto equilibrante en el pool de especies regional y que la riqueza se alcance más rápido en las fincas orgánicas. Por otro lado, cuando los subsidios energéticos en forma de herbicidas y fertilizantes 
reducen la composición a niveles mínimos, y las tierras agrícolas ocupan una parte importante del paisaje, la introducción de nuevas especies se vuelve infrecuente porque el paisaje tiene un ambiente agrícola similar. Ghersa et al. (1994) encontraron que para las plantas el efecto del manejo en determinar la biodiversidad a escala de finca fue más fuerte que el efecto del paisaje debido a que la agricultura convencional anula el efecto del paisaje al incrementar el uso de insumos químicos (Gabriel et al., 2010).

Por otro lado, el efecto de la complejidad del paisaje puede ser más importante en los márgenes que en el interior de las fincas. Que no haya un efecto marcado en la complejidad en el interior de los campos, puede deberse a que la intensificación del área cultivada es más intensa que en los bordes y los márgenes, lo que inhibe la expresión de la diversidad vegetal (Romero et al., 2008; Poggio et al., 2010). A su vez, la dispersión a corta distancia de las semillas de especies de los márgenes puede ocultar el efecto positivo de la complejidad en el interior de las fincas, ya que muchas semillas provienen de los propios campos y se dispersan unos pocos metros (Devlaeminck et al., 2005; Roschewitz et al., 2005).

En el Capítulo 3 se caracterizó la diversidad presente en el Cinturón Hortícola, que fue comparativamente superior a la señalada en sistemas extensivos. Esto se relacionó con la particularidad de la producción hortícola, ya que se desarrolla en pequeñas áreas, es más diversificada y se localiza generalmente en un ámbito periurbano (Archenti et al., 1993; Barsky, 2005). Sin embargo, se planteó que esta heterogeneidad podría diferir entre los distintos tipos de manejo, lo que se reafirma con los resultados aquí presentados. Al tener las fincas hortícolas tamaños reducidos comparados con otras formas de cultivo (Batáry et al., 2011), se reduce la distancia con los ambientes seminaturales incrementando la posibilidad del aporte de propágulos. No obstante, la supervivencia de las especies parece estar más ligada a la intensidad de disturbios generada por las prácticas agrícolas que al paisaje circundante. La mayor diversidad $\gamma$ y $\beta$ encontrada en las fincas orgánicas a entornos similares, sugiere que son más o menos autosuficientes y no dependen tanto de la inmigración de especies de hábitats en paisajes complejos, ya que podrían mantener un importante banco de semillas que persisten en el suelo, lo que garantiza la emergencia de plantas y un aporte de semillas de los márgenes. Estos aspectos son favorecidos por la heterogeneidad de hábitats generada por el parcelamiento. Sin embargo, en las fincas convencionales intensivas la calidad de los hábitats suele disminuir por el alto uso de

Diversidad Vegetal Espontánea en Agroecosistemas Hortícolas de La Plata y su relación con diferentes estilos de Agricultura: Importancia para la sustentabilidad 
insumos y la baja diversidad cultivada, y en los bordes de los alambrados por la aplicación de herbicidas y su homogeneidad cultivada.

El área relevada a partir de las grillas, comprendió unidades del paisaje dentro y fuera de las fincas. Dado que la diversidad $\gamma$ se relaciona con el manejo y la heterogeneidad dentro de las fincas, se puede concluir que el incremento de la intensidad de manejo y una menor diversidad cultivada podrían poner en riesgo la presencia de algunas especies de plantas espontáneas responsables de servicios ecológicos.

Existen antecedentes en los que se ha analizado la complejidad del paisaje a partir de la utilización de variables que describen la composición y diversidad de unidades con diferente tipo de cobertura y se ha analizado su relación con la riqueza de plantas relevadas dentro de los agroecosistemas (Roschewitz et al. 2005; Gabriel et al., 2013). Esta forma de relevamiento y análisis se ha considerado también en este capítulo. Un análisis de mayor detalle requiere la comparación de la composición florística de relevamientos realizados tanto dentro de las fincas como en su entorno, para establecer, por ejemplo, la importancia de los márgenes de las fincas. Además podría analizarse la contribución del arreglo espacial de los elementos del paisaje (configuración) a la diversidad, aunque no es el objetivo y alcance de esta tesis.

\subsection{CONCLUSIONES}

Los resultados presentados en este capítulo confirman que la heterogeneidad de hábitats en las fincas favorece el aumento de la diversidad $\beta$ y $\gamma$ de especies espontáneas. Las fincas orgánicas tuvieron una mayor riqueza $\gamma$ y una mayor diferencia en la composición de especies entre parcelas de cultivo, respecto de las convencionales. La diversidad $\beta$ bajo cubierta fue mayor en los convencionales intensivos, posiblemente por la baja riqueza a nivel $\alpha$ de las parcelas con presencia de diferentes especies pioneras resistentes a las condiciones de alta intensificación que incrementa la variabilidad entre parcelas y dan como resultado una mayor diversidad $\beta$.

La heterogeneidad en las fincas orgánicas se debió al grado de parcelamiento dado por la variedad de componentes estructurales como, parcelas pequeñas cultivadas y no cultivadas. A su vez, aspectos funcionales y taxonómicos de los cultivos que incrementan

Diversidad Vegetal Espontánea en Agroecosistemas Hortícolas de La Plata y su relación con diferentes estilos de Agricultura: Importancia 


\section{Capítulo 6}

la heterogeneidad microambiental podrían contribuir a la diversidad de espontáneas en las escalas analizadas.

En el manejo orgánico se destacó la presencia de especies nativas, perennes, dicotiledóneas y exclusivas o raras. Esto se debió a que representan sistemas de menor disturbio respecto de los sistemas bajo manejos convencionales. En los sistemas orgánicos, persisten plantas con distintas estrategias de crecimiento y en ellos la misma diversidad podría prevenir la invasión de exóticas. Además la agricultura orgánica puede favorecer especies no adaptadas a los sistemas convencionales por ser sensibles a los métodos de control o a la abundancia de $\mathrm{N}$ soluble o altamente disponible.

Las diversidades $\gamma$ y $\beta$ no se relacionaron con las variables que describen la complejidad del paisaje. Esto podría deberse a la similar representatividad de ambientes seminaturales y cultivados que rodean a las fincas de cada tipo de manejo, con lo cual no se encuentran áreas de paisajes simples que se vinculen a una menor diversidad. Por otro lado, el efecto del manejo en determinar la biodiversidad a escala de finca puede ser más fuerte que la complejidad del paisaje debido a que la agricultura convencional anula el efecto del paisaje al incrementar el uso de insumos químicos.

Los beneficios de la producción orgánica estarían dados, en parte, por una mayor calidad y cantidad de hábitats cultivados y parcelas comparadas con los sistemas convencionales. Esto se favorece por la forma de comercialización a través de canales cortos de venta en los que interviene la demanda de los consumidores, los cuales afectan la decisión del agricultor sobre el diseño de su finca. Estos aspectos pueden no ser exclusivos de la producción orgánica y encontrarse por ejemplo, variedad de cultivos en los convencionales.

La tendencia a la homogeneización de los hábitats a través del uso de fertilizantes y herbicidas y la producción de unos pocos cultivos, puede tener un importante impacto sobre la diversidad de plantas espontáneas acompañantes de los cultivos. 


\subsection{BIBLIOGRAFÍA}

Altieri MA. 1999. The ecological role of biodiversity in agroecosystems. Agriculture, Ecosystems and Environment 74: 19-31.

Archenti A, Ringuelet R \& Salva MC. 1993. Los procesos de diferenciación de los productores hortícolas de La Plata. Etnia, $n^{\circ}$ 38/39.

Armengot L, José-María L, Blanco-Moreno JM, Bassa M, Chamorro L \& Sans FX. 2011. A novel index of land use intensity for organic and conventional farming of Mediterranean cereal fields. Agronomy for Sustainable Development 31: 699-707.

Baessler C \& Klotz S. 2006. Effects of changes in agricultural land-use on landscape structure and arable weed vegetation over the last 50 years. Agriculture, Ecosystems and Environment 115: 43-50.

Baležentienè L. 2011. Alpha-diversity of differently managed agro-ecosystems assessed at a habitat scale. Polish Journal of Environmental Studies 20(6):1387-1394.

Barsky A. 2005. El periurbano productivo, un espacio en constante transformación. Introducción al estado del debate, con referencias al caso de Buenos Aires. Revista Electrónica de Geografía y Ciencias Sociales 9(194): 36. Disponible en: http://www.ub.es/geocrit/sn/sn-194-36.htm.

Batáry P, Baldi A, Kleijn D \& Tscharntke T. 2010. Landscape-moderated biodiversity effects of agri-environment management-a meta-analysis. Proceedings of the Royal Society B 278: 1894-1902.

Bavec M \& Bavec F. 2015. Impact of organic farming and biodiversity. En: Blanco J \& YuehHisin Lo \& Roy S (Eds). Biodiversity and ecosystems. Linking structure and function. Intech 658 p.

Benech-Arnold RL, Sánchez RA, Forcellab F, Kruka BC \& Ghersa CM. 2000. Environmental control of dormancy in weed seed banks in soil. Field Crops Research 67: 105-122.

Benton TG, Vickery JA \& Wilson JD. 2003. Farmland biodiversity: is habitat heterogeneity the key? Trends in ecology and evolution 18(4): 182-188.

Borgy B, Gaba S, Petit S \& Reboud X. 2012. Non-random distribution of weed species abundance in arable fields. Weed Research 52: 383-389.

Chase JM. 2003. Community assembly: when should history matter? Oecologia 136: 489498.

Collins W \& Qualset O.1999. Biodiversity in Agroecosystems CRC Press, Boca Raton, Florida. $334 \mathrm{p}$.

Comisión Nacional para el Conocimiento y Uso de la Biodiversidad (CONABIO). 2006-2012. Sitio de malezas de México. Herbario Virtual de CONABIO. Disponible en: http://www.conabio.gob.mx/otros/cgi-bin/herbario.cgi.

Diversidad Vegetal Espontánea en Agroecosistemas Hortícolas de La Plata y su relación con diferentes estilos de Agricultura: Importancia 
Crowder DW, Northfield TD, Gomulkiewicz R \& Snyder W E. 2012. Conserving and promoting evenness: organic farming and fire-based wildland management as case studies. Ecology 93: 2001-2007.

de la Fuente EB, Suárez SA, Ghersa CM \& León RJC. 1999. Soybean weed comunities: relationships whit cultural history and crop yield. Agronomy Journal 91: 234-241.

de la Fuente EB, Suárez SA \& Ghersa CM. 2006. Soybean weed community composition and richness between 1995 and 2003 in the Rolling Pampas (Argentina). Agriculture, Ecosystems and Environment 115: 229-236.

Devlaeminck R, Bossuyt B \& Hermy M. 2005. Seed dispersal from a forest into adjacent cropland. Agriculture, Ecosystems and Environment 107: 57-64.

Edesi L, Jarvan M, Adamson A, Lauringson E \& Kuh, J. 2012. Weed species diversity and community composition in conventional and organic farming: a five-year experiment. Zemdirbyste-Agriculture 99: 339-346.

Evans LT. 1993. Crop Evolution, Adaptation and Yield Evolution. Cambridge University Press, Cambridge. 500 p.

Fahrig L, Girard J, Duro D, Pasher J, Smith A, Javorek S, King D, Lindsay KF, Mitchell S \& Tischendorf L. 2015. Farmlands with smaller crop fields have higher within-field biodiversity. Agriculture, Ecosystems and Environment 200: 219-234.

Ferriol Molina M \& Merle Farinós H. 2012. Los componentes Alfa, Beta y Gamma de la biodiversidad. Aplicación al estudio de las comunidades vegetales. Artículo docente, Universidad Politécnica de Valencia, 10 p. Disponible en: http://riunet.upv.es/handle/10251/16285. Ultimo acceso: agosto de 2018.

Foley JA, DeFries R, Asner GP, Barford C, Bonan G, Carpenter R, Chapin FS, Coe MT, Daily GC, Gibbs HK, Helkowski JH, Holloway T, Howard EA, Kucharik CJ, Monfreda C, Patz JA, Prentice IC, Ramankutty N \& Zinder PK. 2005. Global Consequences of Land Use. Science 309: 570-574.

Forman RTT. 1995. Land Mosaics. The Ecology of Landscapes and Regions. Cambridge University Press, Cambridge, UK. 632 p.

Gabriel D, Roschewitz I, Tscharntke T\& Thies C. 2006. Beta diversity at different spatial scales: plant communities in organic and conventional agriculture. Ecological Application 16 (5): 2011-2021.

Gabriel D, Sait SM, Hodgson JA, Schmutz U, Kunin WE \& Benton TG. 2010. Scale matters: the impact of organic farming on biodiversity at different spatial scales. Ecology Letters 13(7): 858-869.

Gabriel D, Sait SM, Kunin WE, \& Benton TG. 2013. Food production vs. biodiversity: comparing organic and conventional agriculture. Journal of Applied Ecology 50(2): 355364.

Gaston KJ. 1194. Rarity. Chapman \& Hall. London. 163 p.

Diversidad Vegetal Espontánea en Agroecosistemas Hortícolas de La Plata y su relación con diferentes estilos de Agricultura: Importancia para la sustentabilidad 
Ghersa CM, Roush ML, Radosevich SR \& Cordray S. 1994. Co-evolution of agroecosystems and weed management. BioScience 44: 85-94.

Ghersa CM \& Martinez-Ghersa MA. 2000. Ecological correlates of weed seed size and persistence in the soil under different tilling systems: implications for weed management. Field Crops Research 67: 141-148.

Gliessman S. 2001. Agroecología. Processos ecológicos em agricultura sustentável. Seguna Edición. Editora da Universidade Río Grande do Sul, Brasil. 18: 509-538.

Grime JP. 1977. Evidence for the existence of three primary strategies in plants and its relevance to ecological and evolutionary strategy. American Naturalist 111: 1169-1194.

Henckel L, Borger L, Meiss, Gaba S \& Bretagnolle V. 2015. Organic field sustain weed metacommunity in farmland landscapes. Proceeding of de Royal Society B: 282: 20150002.

Hole DG, Perkins AJ, Wilson JD, Alexander IH, Grice PV \& Evans AD. 2005. Does organic farming benefit biodiversity?. Biological Conservation 122: 113-130.

Hughes AR, Byrnes JE, Kimbro DL \& Stachowicz JJ. 2007. Reciprocal relationships and potential feedbacks between biodiversity and disturbance. Ecology Letters 10: 849-864.

Hurrell JA, Ulibarri EA, Delucchi G \& Pochettino ML. 2009. Biota Rioplatense XIV. Hortalizas. Verduras y Hortalizas. Editorial LOLA. 237 p.

Hyvönen T, Ketoja E, Salonen J, Jalli H \& Tiainen J. 2003. Weed species diversity and community composition in organic and conventional cropping of spring cereals. Agriculture, Ecosystems and Environment 97: 131-149.

Jackson LE, Pascual U \& Hodgkin T. 2007. Utilizing and conserving agrobiodiversity in agricultural landscapes. Agriculture, Ecosystems and Environment 121: 196-210.

Koleff P \& Gaston KJ. 2002. The relationships between local and regional species richness and spatial turnover. Global Ecology and Biogeography 11: 363-375.

Krauss J, Klein A, Steffan-Dewenter I \& Tscharntke T. 2004. Effects of habitat area, isolation, and landscape diversity on plant species richness of calcareous grasslands. Biodiversity and Conservation 13: 1427-1439.

Krebs JR, Wilson JD, Bradbury RB \& Siriwardena GM. 1999. The second silent spring?. Nature 400: 611-612.

Liebman M \& Dick E. 1993. Crop rotation and intercropping strategies for weed management. Ecological Application 3: 92-122.

McLaughlin A \& Mineau P. 1995. The impact of agricultural practices on biodiversity.. Agriculture, Ecosystems \& Environment 55: 201-212.

Magurran AE. 1988. Ecological Diversity and Its Measurement. Pinceton University Press, New Jersey. 179 p.

Diversidad Vegetal Espontánea en Agroecosistemas Hortícolas de La Plata y su relación con diferentes estilos de Agricultura: Importancia para la sustentabilidad 
Marshall EJP, Brown VK, Boatman ND \& Lutman PJW. 2003. The role of weeds in supporting biological diversity within crop fields. Weed Research 43: 77-89.

Matson PA, Parton WJ, Power AG \& Swift MJ. 1997. Agricultural intensification and ecosystem properties. Science 277: 504-509.

Palmer MW \& Maurer T.1997. Does diversity beget diversity?. A case study of crops and weed. Journal of Vegetation Science 8: 235-240.

Omezine A \& Teixeira da Silva. 2012. Floristic Biodiversity of weed communities in relation to and conventional in organic farming. Bioremediation, Biodiversity and Bioavailability 6(1): 61-69.

Perronne R, Le Corré V, Bretagnolle V \& Gaba S. 2015. Stochastic processes and crop types shape weed community assembly in arable fields. Journal of Vegetation Science 26: 348-359.

Petit S, Alignier A, Colbach N, Joannon A, Le Coeur D \& Thenail C. 2013. Weed dispersal by farming at various spatial scales. A review. Agronomy for Sustainable Development 33: $205-217$.

Petit S, Munier-Jolain N, Bretagnolle V, Bockstaller C, Gaba S, Cordeau S \& Colbach N. 2015. Ecological intensification through pesticide reduction: weed control, weed biodiversity and sustainability in arable farming. Environmental Management 56(5): 10781090.

Poggio SL, Satorre EH \& de la Fuente EB. 2004. Structure of weed communities occurring in pea and wheat crops in the Rolling Pampa (Argentina). Agriculture, Ecosystems \& Environment 103(1): 225-235.

Poggio SL, Chaneton EJ \& Ghersa CM. 2010. Landscape complexity differentially affects alpha, beta, and gamma diversities of plants occurring in fencerows and crop fields. Biological Conservation 143(11): 2477-2486.

Poggio SL, Chaneton EJ \& Ghersa CM. 2013. The arable plant diversity of intensively managed farmland: Effects of field position and crop type at local and landscape scales. Agriculture, Ecosystems \& Environment 166: 55-64.

Romero A, Chamorro L \& Sans FX. 2008. Weed diversity in crop edges and inner fields of organic and conventional dryland winter cereal crops in NE Spain. Agriculture, Ecosystems and Environment 124 (1-2): 97-104.

Roschewitz I, Gabriel D, Tscharntke T \& Thies C. 2005. The effects of landscape complexity on arable weed arable diversity in organic and conventional farming. Journal of Applied Ecology 42(5): 873-882.

Roschewitz I, Tscharntke T \& Thies C. 2006. Beta diversity at different spatial scales: plant communities in organic and conventional agriculture. Ecological Application 16(5): 2011202. 
Sarandón SJ. 2002. El agroecosistema: un sistema natural modificado. Similitudes y diferencias entre ecosistemas naturales y agroecosistemas. En: Sarandón SJ (Ed.). Agroecología. El camino hacia una agricultura sustentable. Ediciones Científicas Americanas, La Plata. 6: 119-134.

Sarr DA, Hibbs DE \& Huston MA. 2005. A Hierarchical Perspective of Plant Diversity. The Quarterly Review of Biology 80(2): 187-212.

SCDB (Secretaría del Convenio sobre la Diversidad Biológica). 2008. La biodiversidad y la agricultura. Salvaguardando la biodiversidad y asegurando alimentación para el mundo, Montreal. 56 p. Disponible en: HYPERLINK "https://www.cbd.int/doc/bioday/2008/ibd2008-booklet-es.pdf" https://www.cbd.int/doc/bioday/2008/ibd-2008-booklet-es.pdf.

Schneider MK, Lüscher G, Jeanneret P, Arndorfer M, Bailey D, Balács K, Dennis P, Fjellstad W, Friedel JK, Jongman RHG, Kainz M, Moreno G, Paoletti MG, Pointereau P, Sarthou JP, Siebrecht D, Sommaggio J, Wolfrum S \& Herzog F. 2014. Gains to species diversity in organically farmed fields are not propagated at the farm level. Nature communications 5:4151.

Shmida A \& Wilson MV. 1985. Biological determinants of species diversity. Journal of Biogeography 12: 1-20.

Stupino SA, Frangi JL, Sarandón SJ, Arturi MF \& Ferreira AC. 2008. Plant diversity in two farms under organic and conventional management in La Plata, Argentina. A case study. Revista Brasilera de Agroecología, Asociación Brasilera de Agroecología 3(3): 24-35.

Stupino SA, lermanó MJ, Gargoloff NA \& Bonicatto MM. 2014. La biodiversidad en los agroecosistemas. En: Sarandón J \& Flores C (Eds.). Agroecología: Bases teóricas para el diseño y manejo de agroecosistemas sustentables. Colección de libros de cátedra, Secretaria de Asuntos Académicos-Edulp, UNLP.

Suárez SA, de la Fuente EB, Ghersa CM \& León RJC. 2001. Weed community as an indicator of summer crop yield and site quality. Agronomy Journal 93: 524-530.

Sutcliffe OL \& Kay QON. 2000. Changes in the arable flora of central southern England since the 1960s. Biological Conservation 93: 1-8.

Swift MJ, MN Izac \& van Noordwijk M. 2004. Biodiversity and ecosystem services in agricultural landscapes-are we asking the right questions?. Agriculture, Ecosystems and Environment 104: 113-134.

Terradas J. 2001. De la ecofisiología de las plantas a la dinámica de comunidades y paisajes. Omega, Barcelona. 760 p.

Travlos IS, Cheimona N, Roussis I \& Bilalis DJ. 2018. Tillage and fertilization effect on weeds. Frontiers in Environmental Science 6(11): 1-10.

Tscharntke T, Kleijn AM, Kruess A, Steffan-Dewenter I \& Thies C. 2005. Landscape perspectives on agricultural intensification and biodiversity (ecosystem service management. Ecology Letters 8: 857-874. 


\section{Capítulo 6}

van Elsen T. 2000. Species diversity as a task for organic agriculture in Europe. Agriculture, Ecosystems and Environment 77: 101-109.

Vigliola MI. 2007. Manual de Horticutura. Editorial Hemisferio Sur. 235 p.

Weibull AC \& Ostman O. 2003. Species composition in agroecosystems: the effect of landscape, habitat, and farm management. Basic and Applied Ecology 4: 349-361.

Weibull AC, Ostman O \& Granqvist A. 2003. Species richness in agroecosystems: the effect of landscape, habitat and farm management. Biodiversity and Conservation 12: 13351355.

Whittaker RH. 1960. Vegetation of the Siskiyou mountains. Oregon and California. Ecological Monographs 30: 279-338.

Whittaker RJ, Willis KJ \& Field R. 2001. Scale and species richness: Towards a general, hierarchical theory of species diversity. Journal of Biogeography 28: 453-470.

Winqvist C, Ahnström J \& Bengtsson J. 2012. Effects of organic farming on biodiversity and ecosystems services: taking landscape complexity into account. Annals of the New York Academy of Sciences 1249: 191-203. 


\section{CAPÍTULO 7}

\section{DISCUSIÓN GENERAL}

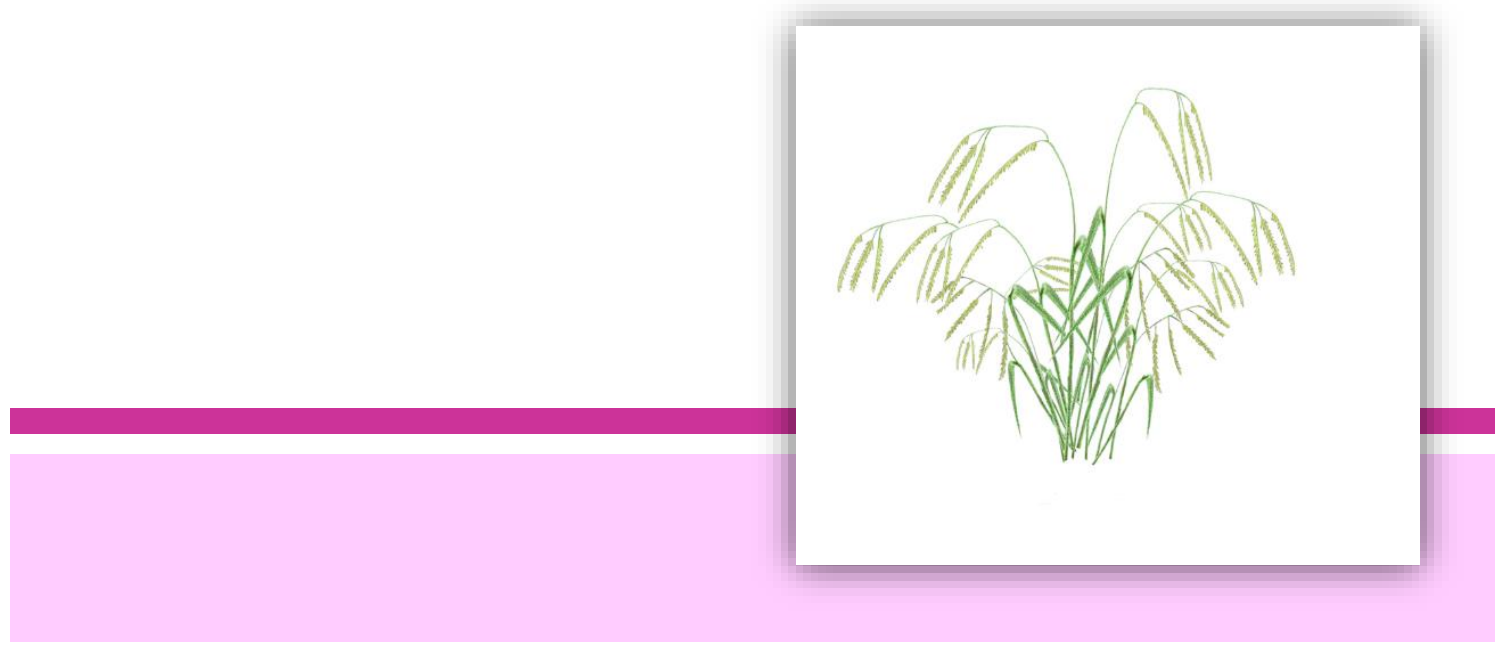




\section{CAPITULO 7}

El secreto no es correr detrás de las mariposas, es cuidar el jardín para que ellas vengan hacia ti.

Mario Quintana (1906-1994). Periodista y escritor.

\section{DISCUSIÓN GENERAL}

\subsection{Introducción}

El modelo de agricultura moderna, ha impulsado un proceso de intensificación basado en el incremento en el uso de los recursos y la aplicación de tecnologías como unos pocos cultivares de alto rendimiento, fertilizantes y pesticidas químicos (Matson, 1997; Altieri \& Nicholls, 2000; Gabriel et al., 2013) (detallado en el Capítulo 1). Esto ha generado la disminución de hábitats a escala de finca y de paisaje (Benton et al., 2003; Foley et al., 2005; Tscharntke et al., 2005), con el consecuente impacto sobre la biodiversidad. Desde hace mucho tiempo, la utilización de diferentes métodos de control tendientes a reducir la vegetación espontánea que crece junto a los cultivos ha sido una práctica habitual. Esto refleja la necesidad de un mejor entendimiento de su importancia en el agroecosistema, lo cual requiere reconocer a la vegetación espontánea desde una mirada diferente al concepto tradicional de malezas, que se definen como plantas que son dañinas para el hombre y sus sistemas productivos (Marzocca, 1979; Radosevich et al., 2007; Stupino et al., 2014).

Desde la mirada de la agricultura sustentable se busca lograr una producción que conserve los recursos naturales, tendiendo a una menor dependencia de insumos externos y optimizando los procesos ecológicos que ocurren en el agroecosistema (Altieri \& Nicholls, 2000; Brussaard et al., 2010). Esto implica el desarrollo de prácticas alternativas de manejo que promuevan la presencia de biodiversidad para el adecuado funcionamiento de los agroecosistemas (Tscharntke et al. 2005; Stupino et al., 2014). En esa dirección, el 
propósito general de esta tesis fue caracterizar la diversidad y composición vegetal espontánea y analizar la influencia que las distintas modalidades de manejo tienen sobre la diversidad en el Cinturón Hortícola de La Plata. Asimismo, se evaluó la influencia del paisaje sobre la diversidad biológica en los distintos sistemas de horticultura locales.

En este capítulo, se discuten los resultados obtenidos en la zona de estudio poniéndolos en el contexto del conocimiento de otras regiones del mundo y de otros tipos de producciones pampeanas. Se evalúan sus implicancias para el manejo de la biodiversidad en los sistemas hortícolas de la zona de la Ciudad de La Plata y sus alrededores.

\subsection{Biodiversidad presente en el Cinturón Hortícola Platense}

La mayoría de los trabajos sobre diversidad espontánea fueron realizados en Europa en sistemas extensivos (Bárberi, 2001; Edesi et al., 2012; Henckel et al., 2015). En Argentina también se han analizado sistemas extensivos (Poggio, 2005; Puricelli et al. 2012; Poggio et al., 2013), y en menor medida se consideró la diversidad de sistemas hortícolas tanto en general como referido a zonas particulares (Stupino et al., 2007; 2008; Fernandez \& Marasas, 2015).

La caracterización de la biodiversidad vegetal espontánea del Cinturón Hortícola, revela su importancia en la región. El relevamiento incluyó 152 especies determinadas, 120 géneros y 40 familias de espontáneas; estos números serían asimismo subestimaciones ya que un $17 \%$ del material no pudo ser determinado (Capítulo 3). El 89\% de las especies tuvo un significativo valor de uso para el hombre, destacándose los usos medicinales y comestibles. Esta biodiversidad se localiza en un total de 40 especies de cultivo relevados. La riqueza encontrada en una superficie total de 178 ha es elevada en comparación a sistemas de monocultivos de gran superficie en áreas comparables de la Pampa Argentina (Poggio et al. 2004; Scursoni et al. 2014). Por ejemplo: Fuente et al. (1999) reportaron 60 especies de espontáneas en un área total de 6000 ha en la Pampa Ondulada; Scursoni et al. (2014), encontraron 114 especies de espontáneas en un área de 22100 ha el sur de la provincia de Buenos Aires. Si bien debe tenerse en cuenta que son diferentes sistemas 
productivos y que los entornos del paisaje son distintos, esta comparación proporciona una idea de la diversidad del Cinturón Hortícola.

\subsection{Influencia de la diversidad cultural}

La actitud del agricultor individual es una de los factores más importantes en determinar la diversidad, ya que es quien la administra a través del diseño y manejo de los cultivos y quien decide que organismos controlar. Pocos trabajos en otras regiones han analizado la percepción que los agricultores tienen acerca de la vegetación espontánea (Stilma et al.; 2009; Kelemer et al., 2013). Las entrevistas realizadas acerca de la percepción que tienen los mismos sobre la vegetación espontánea confirman que, en el Cinturón Hortícola Platense prevalece el concepto tradicional de "maleza", que se ve reflejado en una dominante valoración de las plantas espontáneas como algo perjudicial o negativo por parte de los agricultores (Capítulo 3). Principalmente, predomina el criterio económico de eliminarlas por afectar el rendimiento de los cultivos. Además, se destaca la preocupación por la interferencia en las labores y el criterio estético de mantener el campo "limpio". El reconocimiento del potencial perjuicio ocasionado por la vegetación espontánea, está por encima de los posibles beneficios atribuidos (aspectos positivos). A pesar del alto valor de uso potencial de las especies de plantas encontradas, la mayoría de los agricultores no reconocen un valor de uso. Esto sugiere que el reconocimiento de los beneficios, servicios ecológicos y valores de uso de la vegetación espontánea no se trasladan en prácticas para potenciarlos o aún existe un escaso conocimiento de los mismos. Estos aspectos dan cuenta de la necesidad de que los extensionistas y técnicos trabajen junto a los agricultores para incrementar el conocimiento acerca de los beneficios de la diversidad y que se traduzcan en prácticas concretas de mejoras de sus sistemas productivos. La percepción del agricultor determina una acción de control sobre la vegetación acompañante de los cultivos, con lo cual una valoración negativa pone en riesgo la presencia de plantas y aún más cuando los herbicidas u otras prácticas de control se vuelven menos costosos y de más fácil acceso. No obstante, existen algunas diferencias en la tolerancia a la vegetación espontánea entre los agricultores de distintos estilos de agricultura como se detalla más adelante. 


\subsection{Los diferentes estilos de agricultura del Cinturón Hortícola}

En los estudios realizados en otras regiones se ha encontrado una mayor diversidad en los sistemas orgánicos respecto de los convencionales intensivos, aunque no está claro cuáles son las variables que lo explican, las cuales se atribuyen a la heterogeneidad de hábitats o el menor uso de insumos (Benton et al. 2003; Hole et al., 2005; Henckel et al. 2015). La mayoría de los estudios diferencian de manera teórica los estilos orgánicos y convencionales sólo por el uso o no de insumos químicos sin considerar otras variables de manejo que los caracterizan. A la hora de realizar estudios comparativos sobre la diversidad, debería tenerse en cuenta un mayor número de variables medibles que podrían tener impacto sobre la diversidad (Bengsston, 2005). A su vez, no se ha considerado un nivel de intensidad de manejo, el cual representa un conjunto de prácticas cuantificables que describen la utilización de los insumos, de los recursos y el grado de simplificación de las fincas. Esto puede definir con mayor precisión diferentes estilos de agricultura para poder luego establecer la influencia de distintas variables de manejo sobre la diversidad.

En la zona del Cinturón Hortícola, se han realizado distintas aproximaciones que han tratado especialmente los sistemas convencionales donde se han considerado en mayor medida variables sociológicas y algunas ligadas a la tecnificación del agro (tratadas en el Capítulo 4). En esta tesis, la cuantificación de las variables de manejo incluidas variables sociales, utilizadas en el análisis multivariado permitió establecer una clasificación objetiva en tres formas principales que conforman un gradiente de intensidad de manejo: orgánico, convencional de bajos insumos y convencional intensivo (Figura 7.1). Además de lo referido al uso de químicos que son considerados más frecuentemente para separar los cultivospredios orgánicos de los convencionales se confirmó que existen otras variables medidas que en conjunto permiten precisar las características que definen cada uno de los tipos de cultivo en la zona estudiada. La metodología utilizada resultó adecuada para identificar posteriormente aquellas variables que tienen influencia sobre los colectivos de especies espontáneas presentes en cada tipo de manejo. 


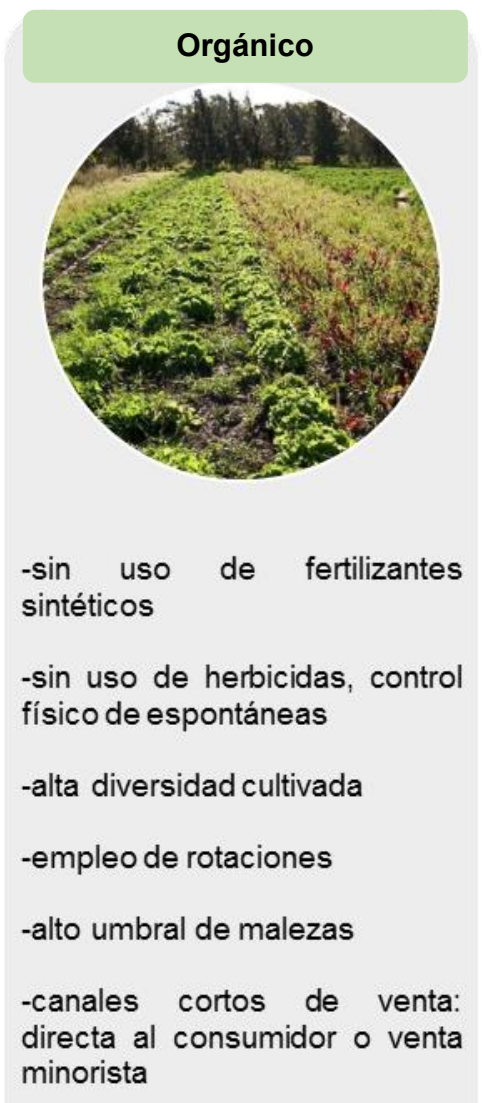

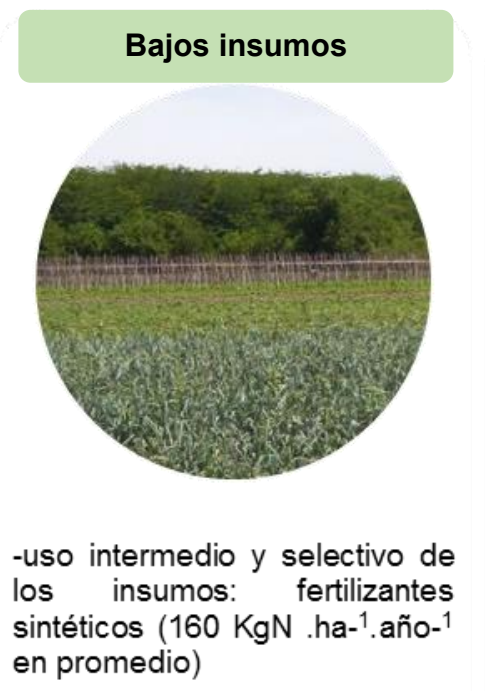

-uso de herbicidas y/o desmalezado físico (este último más frecuente)

-diversidad cultivada intermedia

-distintas estrategias de comercialización: mercados mayoristas y venta minoristas

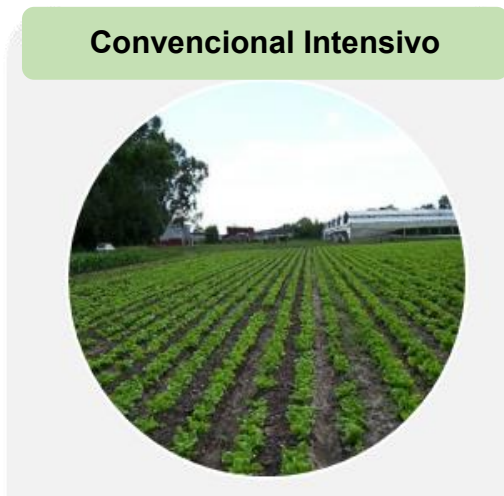

-alto uso de fertilizantes sintéticos (hasta $450 \mathrm{KgN}$.ha- ${ }^{-1}$.año- ${ }^{1}$ )

-alto uso de herbicidas lesterilizadores del suelo

-sin empleo de rotaciones

-bajo umbral de malezas

-alta intensidad de uso del suelo

-pocas especies cultivadas

fincas de mayor superficie $y$ antiguedad

-venta a mercados mayoristas

-la modalidad más extrema del gradiente es el cultivo bajo invernáculo: esterilizadores, riego por goteo y monocultivo contínuo

\section{- Intensidad de manejo +}

Figura 7.1. Principales variables de manejo que describen los estilos de agricultura del Cinturón Hortícola a lo largo de un gradiente de intensidad en el uso de los insumos y los recursos.

El gradiente de intensidad de manejo refleja la heterogeneidad del Cinturón Hortícola. Cada agricultor tiene diferente forma de "hacer y pensar", que los hace diferentes entre sí, pero su agroecosistema se asemeja a aquellos de similar contexto socioeconómicoproductivo y a su lógica personal (cosmovisión) que permite reunirlos en grupos relativamente definidos de tipos de producción pero que en la realidad son segmentos arbitrarios de un gradiente de manejo. Otros factores como el acceso a los insumos, y al mercado influencian en las decisiones del agricultor. 
Las variables detectadas no deben ser necesariamente extrapoladas a otras regiones de estudio, ya que pueden diferir en las variables para clasificar las fincas (Bengtsson et al., 2005).

\subsection{Influencia del manejo a escala parcela de cultivo}

Los resultados presentados en esta tesis muestran que la composición florística y funcional dentro de las parcelas de cultivo se vuelve más homogénea con el aumento de la intensidad de las prácticas de manejo (Capitulo 5). Los cultivos orgánicos presentaron una tendencia a mayor riqueza, equitabilidad y diversidad respecto de los convencionales. Los resultados agregan información a lo encontrado en otros trabajos (Hyvönen et al, 2003; Gabriel et al., 2010; Rahmann, 2011; Edesi et al., 2012; Tuck et al. 2014; Hencklel et al., 2015). En el caso del cultivo de tomate, de importancia por su valor económico, tanto en su versión convencional como orgánica la intensificación del control de malezas (respectivamente: método químico vs mecánico principalmente), condujo a valores similares de diversidad de las acompañantes entre los distintos manejos, aunque la variabilidad fue mayor bajo condiciones orgánicas.

La composición de especies resultó similar entre parcelas bajo un mismo manejo, aunque en valores intermedios de similitud en los diferentes estilos de agricultura. Los cultivos orgánicos y convencionales presentaron un número importante de especies comunes exóticas, anuales y dicotiledóneas, características de sistemas disturbados (Zanin et al., 1997, Suárez et al. 2001; Urban; 2010). Esto coincide con otros estudios en trabajos en sistemas extensivos y hortícolas convencionales (Poggio, 2005; de la Fuente et al, 2006; Cerazo \& Conticello, 2008; Rassam, 2011; Poggio et al., 2013). Sin embargo, en los orgánicos se destacó la presencia de especies raras o exclusivas, especies nativas y de ciclo de vida largo. Esto coincide con otras investigaciones y aporta información adicional para los sistemas hortícolas (van Elsen, 2000; Stupino et al., 2008 Travlos et al., 2018). Esto se corresponde con las características de sistemas productivos menos intensivos, de bajo disturbio, que posibilitan la heterogeneidad florística y funcional intraparcela. Por el contrario, el aumento de la intensidad de las prácticas de manejo se corresponde con una menor diversidad de especies en los cultivos convencionales, que resultó en una composición florística y funcional más homogénea. 
El análisis realizado a nivel de diversidad Alfa refiere a los cultivos hortícolas y extiende los alcances de otras investigaciones previas realizadas a escala de lote (de la Fuente et al., 1999; Suárez et al., 2001; Poggio et al., 2005; Roschewitz et al., 2005).

La diversidad de los cultivos orgánicos es explicada por variables de manejo, que aún están en duda sobre su importancia para el mantenimiento de la biodiversidad vegetal (Gabriel et al., 2010). Un aporte de esta tesis consistió en la identificación de variables que explican la diversidad Alfa y que posiblemente merecerían considerarse en otras regiones. Se comprobó que la vegetación espontánea disminuye con el uso herbicidas y el bajo umbral de malezas o intolerancia del agricultor a las plantas no cultivadas. Adicionalmente se encontró que el uso intensivo de fertilizantes químicos nitrogenados es la variable más importante. Los fertilizantes favorecen la dominancia de especies tolerantes a condiciones de poca luz y de alta disponibilidad de nitrógeno, disminuyendo la riqueza y la diversidad (Pysek \& Leps, 1991; Tang et al., 2013). Si bien, la fertilización puede resultar una práctica adecuada, su uso excesivo, como se observa en la horticultura periurbana (Alconada et al., 2011), puede tener un efecto importante sobre la diversidad, además de ciertos problemas ambientales, con lo cual debería plantearse la utilización en dosis adecuadas de este insumo para no generar el crecimiento de plantas consideradas "problemáticas" por su dominancia e incremento en biomasa. Podrían realizarse prácticas culturales que mejoren la eficiencia en la retención de nutrientes en el suelo. Mantener una mayor cobertura de espontáneas puede ser favorable en este sentido.

La restricción del uso de herbicidas en los cultivos orgánicos podría contribuir a una mayor diversidad de espontáneas respecto de los cultivos convencionales (Gabriel et al., 2010; Roschewitz et al. 2005; Rundlöf et al., 2012). En cambio, los herbicidas pueden ser uno de los filtros más importantes en el establecimiento de espontáneas porque pueden ser selectivos, residuales y resultan en una alta mortalidad en individuos susceptibles (Légère et al., 2005; Ryan et al., 2010). Además, su uso incrementa la presencia de especies comunes tolerantes que aumenta su dominancia, disminuyendo la diversidad. En la zona estudiada, el uso más intensivo de herbicidas se debió a la utilización de productos de amplio espectro y de mayor frecuencia de aplicación como los herbicidas totales (Capítulo 4). Esto demuestra el importante efecto negativo de los herbicidas sobre la riqueza de espontáneas, coincidiendo con otros estudios (Mc Laughlin \& Mineau, 1995; Ghersa \& 
Martínez-Ghersa, 2000). Otra particularidad del control de espontáneas es la percepción del agricultor. El agricultor orgánico es más tolerante a la presencia de espontáneas y ejerce solo un control mecánico, no selectivo sin utilizar fertilizantes químicos, favoreciendo la mayor cobertura de especies de similar abundancia relativa.

Estos resultados permiten establecer mejor las variables de manejo que afectan la diversidad. Para un manejo adecuado, tendiente a la conservación debería contemplarse ajustar las dosis de fertilizantes y reducir el uso de herbicidas. Para esto último, Sánchez Vallduví \& Sarandón (2014) proponen reemplazar la idea de "control" por la de "manejo agroecológico" de la vegetación espontánea dentro de niveles tolerables con la idea de disminuir las interacciones negativas de las poblaciones de vegetación espontánea, pero a la vez, conservando u optimizando sus roles positivos en el agroecosistema. Distintas estrategias pueden ser implementadas como, rotaciones, cultivos de cobertura, labranzas y manejo de la fecha de siembra. Entendiendo al problema de la vegetación espontánea desde un enfoque de sistemas, las prácticas agrícolas pueden ser dirigidas a prevenir la interferencia sobre los cultivos y controlar la colonización o el establecimiento de las especies. El estudio de la dinámica de las poblaciones presentes, son elementos indispensables para el desarrollo de estrategias de manejo que tiendan a optimizar las interacciones positivas y minimizar las negativas.

\subsection{Escalas de diversidad Beta, Gamma y paisaje}

Esta investigación ha presentado algunas evidencias a favor de la heterogeneidad ambiental como determinante de la diversidad a nivel Beta y Gama. Se comprobó que las fincas orgánicas tuvieron una mayor diversidad Beta y Gamma. Los resultados encontrados extienden el entendimiento de los sistemas hortícolas coincidiendo con los resultados de numerosos estudios realizados en otras regiones (van Elsen, 2000; Hyvonen et al., 2003; Roschewitz et al. 2005; Gabriel et al., 2010; Edesi et al., 2012; Henckel et al., 2015; Gabriel et al., 2016).

Tanto la mayor riqueza de especies cultivadas y de parcelas en las fincas orgánicas respecto de las intensivas explicaron una mayor diversidad Beta y Gamma. Una cierta combinación de especies cultivadas puede promover, respecto de un monocultivo, una 
mayor presencia de especies asociadas, al incluir especies de cultivo que por sus características generen condiciones favorables para el establecimiento de espontáneas (Palmer \& Mauler, 1997). Además, las características taxonómicas y funcionales de los cultivos resultan importantes para generar heterogeneidad microambiental. Indirectamente, las prácticas de manejo que difieren entre las distintas especies de cultivo contribuyen a generar heterogeneidad.

Las fincas orgánicas resultan en un diseño y estructura más heterogénea en cuanto a la diversidad planeada. En cambio, el efecto de homogeneización de los hábitats a través del uso de fertilizantes y herbicidas y un esquema de rotación menos diverso, se asocia con sistemas intensivos que han mostrado disminuir la riqueza de espontáneas, al seleccionar pocas especies dominantes con requerimientos similares al cultivo (Benton, 2003; Poggio et al., 2004; Borgi et al., 2012).

Al igual que en el nivel Alfa, a escala Gamma se comprobó que las fincas orgánicas promueven una mayor riqueza de plantas nativas, perennes y dicotiledóneas, atribuidos a las características de sistemas menos disturbados. En niveles de disturbio intermedios coexisten especies de rápido crecimiento generalistas con especies competitivas dominantes de crecimiento lento (Armengot et al., 2012). A su vez, la mayor diversidad puede disminuir el efecto del disturbio y generar resistencia a la invasión de exóticas, lo que explica un mayor número de nativas (Hugues et al., 2007). Puede, además, generar una riqueza taxonómica de propágulos y una variedad en los efectos sobre la intensidad de la luz captada por las plantas, potenciando una diversidad de plantas emergentes.

Se comprobó que las fincas orgánicas presentan más especies espontáneas raras o exclusivas a escala Gamma, coincidiendo con el nivel Alfa. Estos resultados son coincidentes con otros trabajos (van Elsen, 2000; Stupino et al., 2008; Travlos et al., 2018). El incremento en el número de especies raras o poco frecuentes puede corresponderse con la mayor tasa de recambio entre parcelas encontrada en los sistemas orgánicos (alto Beta).

La modalidad de invernáculo se correspondió con la forma de manejo más intensiva, donde el uso de esterilizadores del suelo y las condiciones favorables, predisponen a la aparición de un bajo número de especies resistentes de baja frecuencia a nivel Alfa y que 
incrementan la diversidad Beta, es decir las diferencias entre cultivos en la composición florística.

La diversidad de espontáneas decreció con el incremento de la intensidad de manejo y las variables que representan una mayor heterogeneidad. Uno de los aportes importantes de esta tesis es que, tanto los insumos como la heterogeneidad, explicaron la mayor diversidad. Esto contribuye a llenar un vacío acerca de las variables que explican una mayor diversidad en los sistemas orgánicos, donde se ponía en cuestión cuál de los factores era más importante (Krebs et al. 1999; Hole et al, 2005; Benton et al., 2003). En esta tesis se confirma que además del no uso de químicos, como se define un estilo de agricultura orgánico, también es importante la mayor heterogeneidad de hábitats, considerando diferentes escalas espaciales.

La mayor diversidad de cultivos podría atribuirse a que el agricultor tiene una lógica de producción orgánica que busca diversificar el sistema para favorecer procesos ecológicos. A su vez, la exigencia de una rotación programada de los cultivos es establecida en las normativas orgánicas (IFOAM, 2007), lo cual contribuye a explicar el cultivo de varias especies. No obstante, esto no es necesariamente una regla que explica su mayor riqueza de cultivos, ya que el agricultor podría ser un empresario de monocultivo orgánico, tal como sucede en otras regiones del mundo o que mantenga la misma lógica de mercado y técnicas que la convencional. Asimismo, los convencionales podrían tener un mayor número de cultivos, con lo cual la actitud del agricultor es un factor importante en determinar la diversidad a escala de finca ya que es quien decide qué y cómo cultivar. En la zona de La Plata y alrededores, la relación observada de las fincas orgánicas respecto de determinadas vías de comercialización, sugiere que la mayor diversificación de las mismas puede estar influenciada, en parte, por el destino de sus productos. Al no existir un mercado propio mayorista orientado a los productos orgánicos, el agricultor debe orientarse hacia canales de comercialización de carácter más diverso (canales cortos de venta) lo que determinaría la necesidad de una mayor oferta de productos para satisfacer la demanda. Actualmente, el mercado es un condicionante importante en la zona, donde el precio del producto se rige por la calidad, el cual se obtiene con el empleo de un alto uso de agroquímicos para lograr la "perfección". A su vez, los productos orgánicos certificados tienen costos adicionales de certificación. Con lo cual debería gestionarse un mayor orden en la cadena de 
comercialización, con mayor desarrollo de mercados diversificados, y revalorización de los productos orgánicos $\mathrm{u}$ agroecológicos. La tendencia actual a reducir el número de cultivos y variedades respondiendo a un mercado mayorista puede tener importante consecuencias para la biodiversidad en el Cinturón Hortícola.

La incorporación de la escala del paisaje permitió discriminar la relevancia del efecto del manejo sobre la diversidad en las fincas. Pocos estudios, han evaluado la relación entre la riqueza local de especies espontáneas, el manejo y el contexto del paisaje (Petit et al., 2015; Henckel et al., 2015). En esta tesis, las diversidades Gamma y Beta encontradas dentro de las fincas no se relacionaron con las métricas analizadas del entorno del paisaje. Esto coincide con algunos autores que encontraron un efecto menor del contexto del paisaje en la composición y riqueza de especies a escala de finca (Weibul \& Östman, 2003; Krauss et al., 2004, Armengot, 2011). Las proporciones de parches seminaturales y cultivados fueron similares, con los cuales los niveles de complejidad y los entornos resultaron parecidos para las fincas de distinto manejo. No obstante, desde los inicios de los relevamientos a la actualidad se ha incrementado en el Cinturón Hortícola la cobertura de invernáculos (Garcia, 2015) y algunas producciones han sido abandonadas, con lo cual debería replicarse este relevamiento a un mayor número de fincas y analizar los posibles cambios temporales.

\subsection{Posibilidades de conversión a sistemas de base agroecológica}

Las fincas orgánicas del Cinturón Hortícola son ejemplos de sistemas que reducen el uso de insumos, donde no se usan fertilizantes sintéticos ni pesticidas, e implementan rotaciones de cultivo y pueden incrementan la heterogeneidad a escalas temporales y espaciales. Esto puede contribuir a mantener la biodiversidad (Benton, 2003; Hole, 2005). Sin embargo, se suele confundir la producción orgánica con la agroecológica. A pesar de que ambos términos se refieren a procesos de producción libres de agroquímicos nocivos para la salud del consumidor y que, por lo tanto, utilizan insumos naturales, no representan lo mismo. Los productos ofrecidos como orgánicos certificados han seguido reglamentaciones específicas para su producción. Los sistemas de base agroecológica se diferencian en no seguir un protocolo determinado para obtener una certificación para su comercialización. La Agroecología se basa en la aplicación de principios básicos de 
ecología al diseño y manejo de agroecosistemas sostenibles (Gliessman, 2001). Esto Incluye la conservación de recursos naturales y agrícolas (agua, capital, energía, suelo, y variedades genéticas), el uso de recursos renovables, la minimización del uso de productos tóxicos, el manejo adecuado de la biodiversidad, la maximización de beneficios a largo plazo, y la conexión directa entre agricultores. La agroecología implica un enfoque holístico, centrado no sólo en la producción, sino también en la sostenibilidad del sistema productivo, el respeto al medio ambiente y los aspectos socioeconómicos. No promulga necesariamente la prohibición estricta del uso de insumos sintéticos. Por lo tanto, bajo esta mirada de los agroecosistemas y en base a los resultados obtenidos en la tesis emerge la necesidad de diseñar sistemas sustentables tendientes a conservar la biodiversidad y hacer un uso racional de la misma en el Cinturón Hortícola de La Plata.

En Europa se han desarrollado Esquemas Agro-ambientales (EAA), donde los agricultores reciben un incentivo económico para mejorar los beneficios de la biodiversidad a través de implementar prácticas de menor impacto local (Gabriel et al., 2010). Si bien los contextos son diferentes a la realidad argentina, puede ser factible introducir algún programa que permita la conversión hacia sistemas agroecológicos que deberán ser acompañados por políticas y legislaciones adecuadas. Un aspecto a tener en cuenta es que la efectividad de un programa puede tener que ver con la escala espacial y los grupos de organismos que se requieren conservar (Bengtsson et al., 2005; Fuller et al., 2005). Un paso inicial hacia esta alternativa implica conocer qué especies se encuentran para saber qué se desea conservar y como puede impactar las distintas prácticas de manejo. Estos aspectos que emergen de los resultados de la tesis pueden guiar la toma de decisiones de manejo que garanticen el mantenimiento de la biodiversidad y los servicios ecológicos asociados, en el marco de la sustentabilidad.

Por ejemplo, la caracterización de la flora a nivel zonal contribuye a conocer más acerca de las especies que son fuente de alimento o refugio de otros organismos que integran las tramas tróficas de los sistemas de cultivo. Esto representa un aporte al avance en investigaciones de la zona orientadas en este sentido (Dubrovsky Berensztein et al., 2017; Paleologos et al., 2008) ya que establece el valor ecológico aplicado de la biodiversidad. 
7.8 Marco conceptual ecológico para comprender los cambios, estados y tendencia de los ecosistemas y factores que influyen sobre la diversidad en los ecosistemas hortícolas estudiados útiles para el diseño de sistemas sustentables

Como señalan Hobbs et al. (2009), los ecosistemas están sometidos a cambios bióticos (extinción de nativas o invasión de exóticas) y abióticos (por ejemplo uso de la tierra o climático) o una combinación de ambos. Dados estos cambios, pueden encontrarse aquellos ecosistemas que desarrollan cualidades híbridas o aquellos denominados ecosistemas nóveles que se han modificado en mayor magnitud y la composición y abundancia de especies y/o sus funciones han cambiado notoriamente respecto del ecosistema " original" (histórico) y se sostienen sin intervención humana (Figura 7.2).

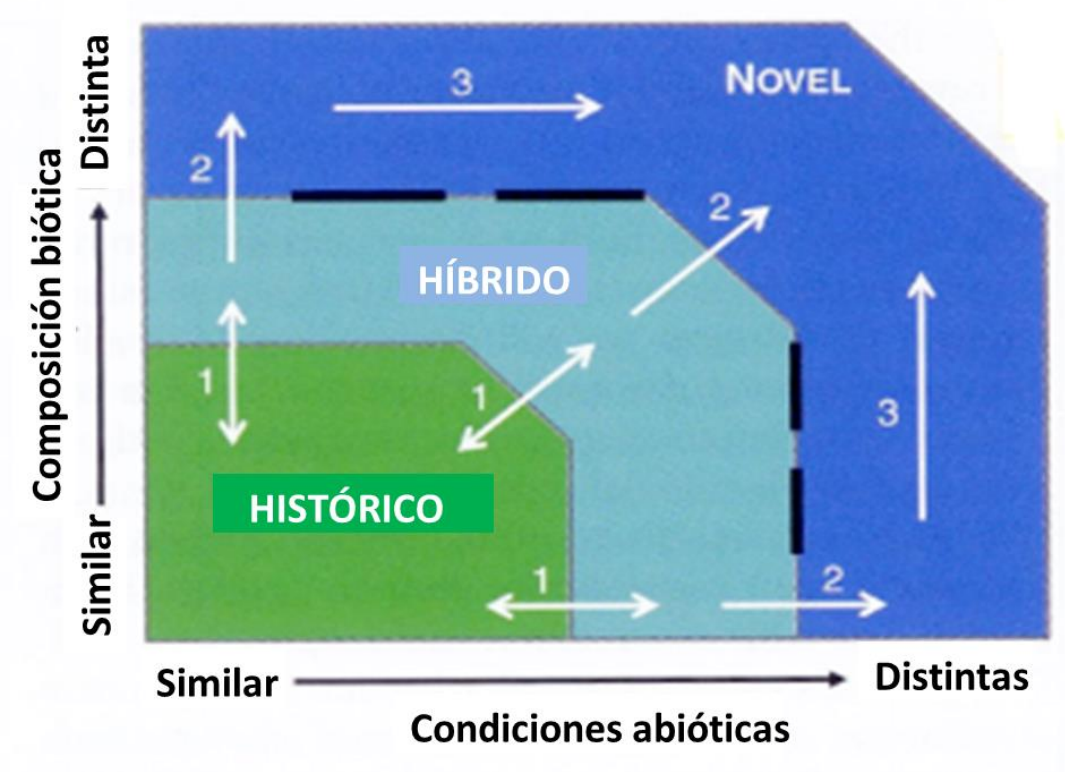

Límite de Restauración

Figura 7.2. Tipos de ecosistemas bajo distintos niveles de cambio biótico o abiótico. Un ecosistema histórico permanece dentro del rango histórico de variabilidad; uno híbrido es biótica y lo a bióticamente distinto a su estado histórico. Las flechas representan posibles direcciones de cambio: 1- los cambios de históricos a híbridos son reversibles, 2- los cambios de históricos o híbridos a ecosistemas noveles son irreversibles, 3- dentro de los ecosistemas noveles es posible que se produzcan cambios bióticos o abióticos adicionales. Fuente: Hobbs et al. (2009). 
Los paisajes culturales, aquellos que manifiestan rasgos de la actividad humana suelen contener una variedad de ecosistemas naturales, seminaturales y producto del uso humano de la tierra con distintas finalidades (Figura 7.3). Entre ellos, los agroecosistemas son ecosistemas que han sido modificados para obtener un producto de interés económico. A pesar de su apariencia simple, son entidades complejas donde sus componentes interactúan en el espacio y tiempo, y en el cual el efecto del manejo sobre la diversidad no puede separarse del sistema en sí mismo y del contexto socioeconómico. Las tareas involucradas en el cultivo, particularmente convencional, empujan el ecosistema hacia un nuevo estado de comunidades biológicas y a suelos deteriorados entre otros (Frangi et al., 2003). El manejo cada vez más intensivo retrotrae a algo comparable a estadios sucesionales "seminaturales" más tempranos e inestables y, en algunos casos, la magnitud de los cambios provocados por el manejo pueden llevar a nuevos caminos de cambios cualitativos y/o cuantitativos composicionales y físicos cuya eventual rehabilitación a una mejor condición, mediante respuesta natural e intervención humana, puede verse comprometida si se superan umbrales bióticos y abióticos que ya no permiten una respuesta resiliente. En éste último caso se pueden establecer ecosistemas noveles en el que la composición de especies es diferente del ecosistema original e independiente ya de la intervención humana. Estos ecosistemas nóveles con un origen que puede relacionarse a los orígenes de la agricultura son un desafío al intelecto en cuanto plantean un gran debate entre las cuestiones de conservación de la biodiversidad espontánea ancestral y aquella biodiversidad más reciente que va reemplazando a las denominadas nativas 0 autóctonas o modificando cuantitativamente la estructura de la comunidad. Por lo tanto, las opciones de gestión varían según el grado de cambio en el que se presentan los agroecosistemas y la presencia de umbrales de intensidad de manejo que podrían permitir o no la acción sucesional natural, la rehabilitación y mucho menos probablemente la restauración. El conocimiento ecológico de los agroecosistemas y los factores naturales y de manejo que afectan su composición biológica, su funcionamiento, y su estabilidad, son una base sustancial para una gestión que conduzca a los objetivos productivos y de conservación que se hayan establecido. 


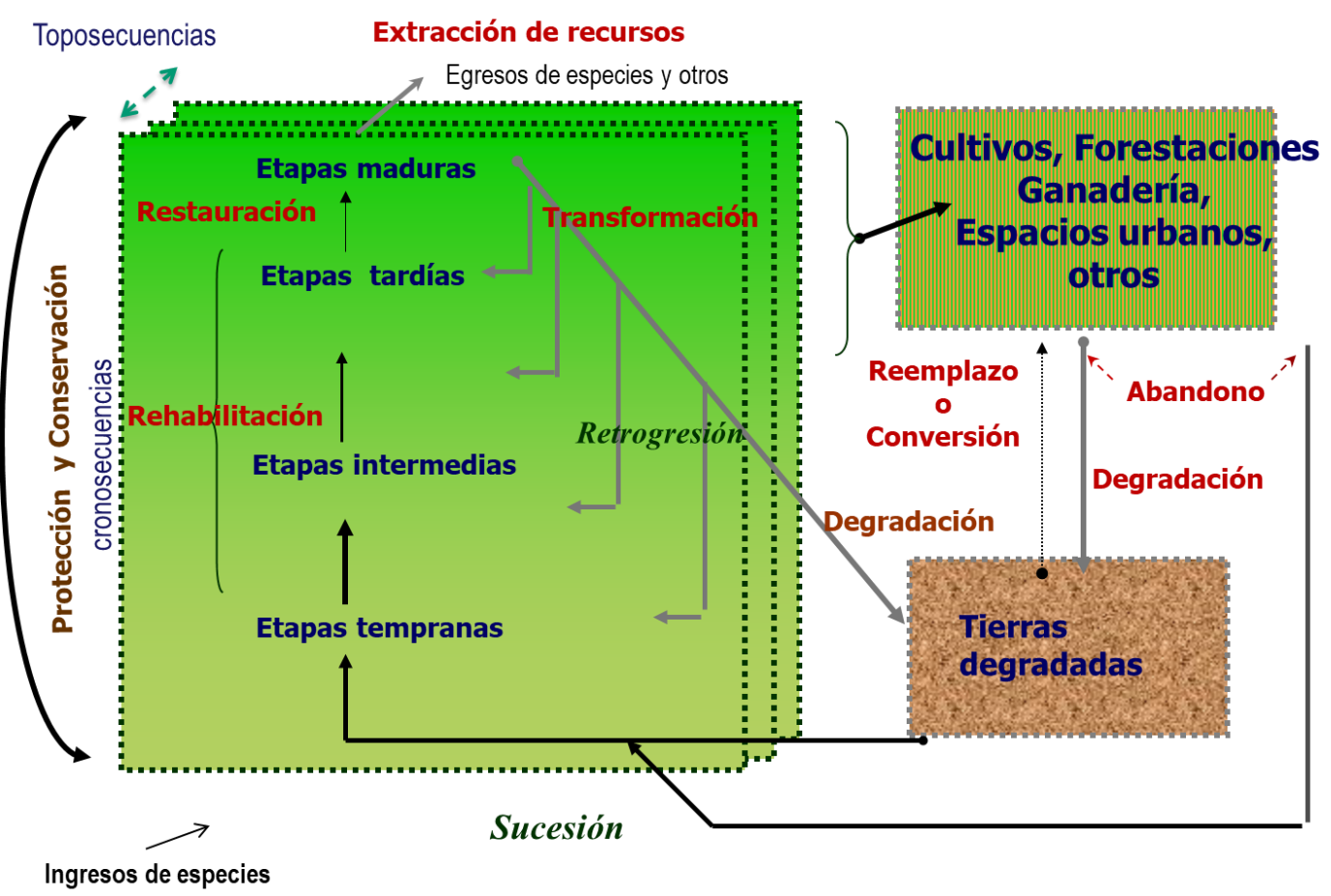

Figura 7.3. Modelo que permite reconocer el conjunto de estrategias de uso de la tierra que incluyen asimismo respuestas espontáneas de la naturaleza. La intensidad del uso de la tierra es importante en el nivel de cambio en los ecosistemas y la posibilidad de respuesta resiliente espontánea, acompañada o no por intervenciones humanas, o la degradación o cambio a nuevos ecosistemas (ecosistemas noveles) que traspasaron umbrales ambientales y bióticos que hacen que el ecosistema ya no dependa de la intervención humana. Fuente: modificado de Frangi et al. (2003). Definición de estrategias de manejo según Lugo (1988).

En el marco conceptual planteado precedentemente, esta tesis se ha enfocado en el estudio de las plantas acompañantes de los cultivos, y la diversidad a distinta escala, vinculada a los tipos de manejo en la zona del Cinturón Hortícola Platense. Se ha logrado determinar objetivamente cuales son los principales factores que caracterizan los tres estilos de cultivo hortícola en las vecindades de La Plata. Asimismo, se han reconocido las relaciones entre distintos factores de manejo y algunos de orden socioeconómico que operan dentro de cada tipo de manejo y la diversidad de plantas acompañantes de los cultivos, avanzando en la identificación de los factores que explican la biodiversidad en diferentes escalas espaciales y estilos de agricultura. El análisis bibliográfico comparado con el estado del conocimiento en agroecosistemas comparables de la región bonaerense y otras partes del mundo, ha permitido una profundización de la interpretación de las posibles causas de las relaciones observadas en el Cinturón Hortícola de La Plata y al mismo tiempo advertir su significado en un contexto agroecosistémico, ecológico y 
geográfico más amplio. Los resultados obtenidos, analizados en un marco teórico ecológico han permitido avanzar en el entendimiento de las características de los sistemas hortícolas y de establecer algunas pautas de respuestas esperadas ante ciertas intervenciones de manejo. A modo de síntesis, en la figura 7.4 se indican los factores analizados que pueden tener influencia sobre la diversidad.

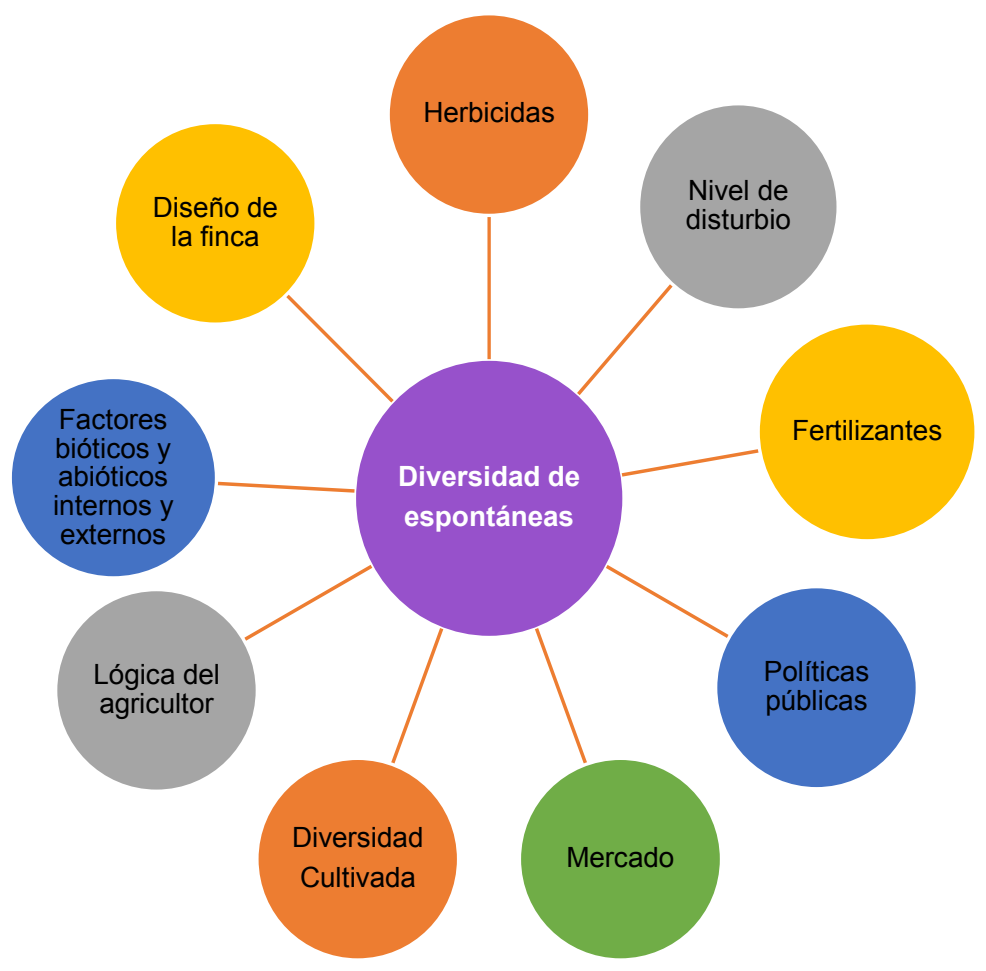

Figura 7.4. Síntesis de los factores que tienen mayor influencia sobre la biodiversidad de especies espontáneas que deberían ser considerados en relación a objetivos de manejo que propendan a una mayor sustentabilidad del Cinturón Hortícola de La Plata.

A modo de breve explicación de lo indicado en éste capítulo puede puntualizarse que:

- El área que comprende el Cinturón Hortícola de La Plata presenta una heterogeneidad dada por un tipo de producción diversificada en una zona periurbana, y con fincas de pequeñas dimensiones y distintas formas de manejo. Presenta una importante 
biodiversidad que resulta comparativamente mayor a sistemas tendientes al monocultivo bajo prácticas estandarizadas que tienden a la homogenización.

- La lógica y percepción que tiene el agricultor sobre la vegetación espontánea influye en la conservación de la diversidad. En el Cinturón Hortícola predomina el concepto tradicional de malezas pero con diferencias entre los tipos de agricultores, siendo los orgánicos más tolerantes a la presencia de plantas.

- Existe entre los agricultores un escaso conocimiento de las funciones positivas de las plantas y su valor utilitario. Los beneficios de las plantas, cuando son reconocidos, no se traducen en una práctica para potenciarlos. El predominio de la percepción negativa de la vegetación espontánea, impulsa la decisión de control, y podría poner en riesgo la biodiversidad, especialmente cuando los insumos se vuelven menos costosos.

- Los diferentes estilos de agricultura presentan diferentes estrategias en el uso de los insumos, recursos y grado de simplificación de las fincas, pasando de sistemas menos disturbados, de baja intensidad de manejo a aquellos más intensivos.

- Existen diferentes variables que diferencian a los manejos orgánicos y convencionales además del uso o no de herbicidas y fertilizantes sintéticos. Aquellas variables tiene que ver con, la riqueza de especies cultivadas y su manejo (empleos de rotaciones, incorporación de períodos de descanso), la percepción del agricultor acerca de la vegetación espontánea y la forma de comercialización.

- Aquellas fincas cuyos agricultores presentan similar situación socioeconómica, forma de producir, y lógica personal, se reconocen como un grupo dentro de las categorías establecidas. En los agricultores de bajos insumos, principalmente, el acceso a los mercados y a los insumos condicionan las decisiones de manejo.

- La tendencia actual es hacia la simplificación de los agroecosistemas y la especialización relativa en pocos cultivos e intensivos en insumos, tal como se observa en los sistemas convencionales intensivos. Sin embargo, en el Cinturón Hortícola Platense se mantienen 
sistemas menos intensivos como los orgánicos y los de bajos insumos, más diversificados, que se diferencian del modelo predominante.

- Los diferentes estilos de agricultura, que surgen de la combinación de ciertas prácticas de manejo presentan diferente efecto sobre la diversidad de plantas acompañantes de los cultivos, en las distintas escalas de análisis.

- Los cultivos sometidos a menor régimen de disturbio, como los orgánicos mantienen una mayor riqueza y diversidad de especies dada por la ausencia en el uso de herbicidas y fertilizantes sintéticos y una mayor tolerancia del agricultor a la presencia de plantas espontáneas. En cambio, las prácticas de manejo intensivas, como el alto uso de fertilizantes nitrogenados y herbicidas de origen sintético y, las labores tendientes a mantener baja la cobertura de plantas se asocian con una menor riqueza y diversidad de plantas en las parcelas de cultivos (escala Alfa).

- Las características de sistemas menos intensivos posibilitan la heterogeneidad florística y funcional intraparcela, con mayor representatividad de especies raras, nativas y de ciclo de vida largo. Por el contrario, el aumento de la intensidad de las prácticas de manejo se corresponde con una menor diversidad de especies que resulta en una composición florística y funcional más homogénea.

- La conservación de las especies debe garantizarse a través de un diseño que implique una riqueza taxonómica y funcional de los cultivos y de parcelas. La heterogeneidad dada por la mayor riqueza de cultivos y parcelas cultivadas de las fincas promueve una mayor diversidad de plantas dentro del área cultivada y un mayor recambio de especies entre las parcelas (escalas Gamma y Beta).

- La diversidad cultivada del Cinturón Hortícola está influenciada por la forma de comercialización. La tendencia a producir grandes volúmenes de un mismo cultivo a través un mercado mayorista pone en riesgo el mantenimiento de la diversidad de plantas espontáneas. 
- El paisaje no se relaciona con la diversidad a escala Gamma, siendo similar el entorno de las fincas orgánicas y convencionales, con semejante proporción y tipo de unidades del paisaje cultivadas y seminaturales.

- El cultivo bajo invernáculo representa el mayor grado de intensificación del manejo, y mantiene baja diversidad de especies. La tendencia actual a incrementar la superficie bajo cubierta podría modificar la relación entre la complejidad del paisaje y la diversidad de especies a escala Gamma.

\subsection{Reflexión final}

El desafío de avanzar hacia una agricultura sustentable implica lograr unir los esfuerzos de conservación con los beneficios sociales y económicos. Una parte importante de la biodiversidad que se desea conservar se encuentra en los agroecosistemas. Por lo tanto, asegurar el mantenimiento de la misma debe ser un compromiso de quienes gestionan los sistemas productivos (agrónomos, horticultores, extensionistas) y quienes generan políticas públicas y un tema de interés general.

Los conocimientos derivados de esta tesis, representan insumos a otras investigaciones relacionadas con la temática, la gestión y diseño de agroecosistemas sustentables, y el desarrollo de políticas públicas. Para incrementar la biodiversidad del paisaje se necesitan agricultores con participación activa, formados e interesados en prácticas ambientalmente racionales como así también profesionales que acompañen estas transformaciones en base al desarrollo de tánicas de manejo fundadas en conocimiento científico actualizado. Por lo tanto, esta tesis podría ser de interés para las unidades de investigación agronómica universitarias, el Instituto Nacional de Tecnología Agropecuaria (INTA), profesionales asesores de los agricultores, y otras instituciones y actores intervinientes en la gestión de agroecosistemas hortícolas. 


\section{10 BIBLIOGRAFÍA}

Alconada M, Cuellas M, Poncetta P, Barragán S, Inda E \& Mitidieri A. 2011. Fertirrigación en cultivo de tomate protegido: Nutrición nitrogenada. Efectos en el suelo y en la producción. Horticultura Argentina 30(72): 5-13.

Altieri M \& Nicholls Cl. 2000. Agroecología. Teoría y práctica para una agricultura sustentable. Serie Textos Básicos para la Formación Ambiental. PNUMA. Red de Formación Ambiental para América Latina y el Caribe, México. 250 p.

Armengot L, José-María L, Blanco-Moreno JM, Bassa M, Chamorro L \& Sans FX. 2011. A novel index of land use intensity for organic and conventional farming of Mediterranean cereal fields. Agronomy for Sustainable Development 31: 699-707.

Bàrberi P, Bonari E, Mazzoncini M, García-Torres L, Benites J \& Martínez-Vilela. 2001. Weed density and composition in winter wheat as influenced by tillage systems. Conservation agriculture, a worldwide challenge. En: Proceedings of the First World Congress on Conservation Agriculture, Spain, 1-5 October, Vol 2: 451-455.

Bengtsson J, Ahnstrom J \& Weibull AC. 2005. The effects of organic agriculture on biodiversity and abundance: a meta-analysis. Journal of Applied Ecology 42: 261-269.

Benton TG, Vickery JA \& Wilson JD. 2003. Farmland biodiversity: is habitat heterogeneity the key? Trend in ecology and evolution 18(4): 182-188.

Borgy B, Gaba S, Petit S \& Reboud X. 2012. Non-random distribution of weed species abundance in arable fields. Weed Research 52: 383-389.

Brussaard L, Caron P, Campbell B, Lipper L, Mainka S, Rabbinge R, Babin D \& Pullman M. 2010. Reconciling biodiversity conservation and food security: scientific challenges for a new agriculture. Current Opinion in Environmental Sustainability 2: 34-42.

Cerazzo B \& Conticello L. 2008. Comunidades de malezas en cultivos en la provincia de Neuquén (Argentina). Boletín de la Sociedad Argentina de Botánica 43: 121-135.

de la Fuente EB, Suarez SA, Ghersa CM \& León RJC. 1999. Soybean weed communities: relationships whit cultural history and crop yield. Agronomy Journal 91: 234-241.

de la Fuente EB, Suarez SA \& Ghersa CM. 2006. Soybean weed community composition and richness between 1995 and 2003 in the Rolling Pampas (Argentina). Agriculture, Ecosystems and Environment 115: 229-236.

Dubrovsky Berensztein N, Mónica Ricci M, Polack LA \& Marasas M. 2017. Control biológico por conservación: evaluación de los enemigos naturales de Brevicoryne brassicae (Hemiptera:Aphididae) en un manejo agroecológico de producción al aire libre de repollo (Brassica oleracea) del Cinturón Hortícola de La Plata, Buenos Aires, Argentina. Revista de la Facultad de Agronomía (La Plata) 116(1): 141-154. 
Edesi L, Jarvan M, Adamson A, Lauringson E \& Kuht J. 2012. Weed species diversity and community composition in conventional and organic farming: a five-year experiment. Zemdirbyste 99: 339-346. Disponible en: http://www.vkm.no/dav/dda3cb51f7.pdf.

Fernández V \& Marasas M. 2015. Análisis comparativo de la riqueza de especies y familias botánicas en sistemas de producción hortícola familiar del Cordón Hortícola de La Plata (CHLP), Provincia de Buenos Aires, Argentina. Su importancia para la transición agroecológica. Revista de la Facultad de Agronomía 114(1): 15-29.

Foley JA, DeFries R, Asner GP, Barford C, Bonan G, Carpenter R, Chapin FS, Coe MT, Daily GC, Gibbs HK, Helkowski JH, Holloway T, Howard EA, Kucharik CJ, Monfreda C, Patz JA, Prentice IC, Ramankutty N \& Zinder PK. 2005. Global Consequences of Land Use. Science 309: 570-574.

Frangi JL, Arturi MF, Goya JF, Vaccaro S, Oliveri NJ \& Píccolo GA. 2003. Lineamientos para el manejo de Capueras del centro sur de Misiones. Estación experimental agropecuaria Cerro Azul. Boletín técnico n 5 .

Fuller RJ, Norton LR, Feber RE, Jhonson PJ, Chamberlain DE, Joys AC, Mathews F, Stuart RC, Townsend MC, Manley WJ, Wolfe MS, Mac Donald WC \& Firbank LG. 2005. Benefits of organic farming to biodiversity vary among taxa. Biology Letters 1: 431-434.

Gabriel D, Sait SM, Hodgson JA, Schmutz U, Kunin WE \& Benton TG. 2010. Scale matters: the impact of organic farming on biodiversity at different spatial scales. Ecology Letters 13(7): 858-869.

Gabriel D, Sait SM, Kunin WE \& Benton TG. 2013. Food production vs. biodiversity: Comparing organic and conventional agriculture. Journal of Applied Ecology 50: 355364.

García M. 2015. Horticultura de la Plata (Buenos Aires). Modelo productivo irracionalmente exitoso. Revista de la facultad de Ciencias Agrarias y Forestales 114 (1): 190-201.

Gliessman S. 2001. Agroecología. Processos ecológicos em agricultura sustentável. Segunda Edición. Editora da Universidade Río Grande do Sul, Brasil. 652 p.

Henckel L, Borger L, Meiss, Gaba S \& Bretagnolle V. 2015. Organic field sustain weed metacommunity in farmland landscapes. Proceeding of the Royal Society B: 282: 20150002. Hole DG, Perkins AJ, Wilson JD, Alexander IH, Grice PV \& Evans AD. 2005. Does organic farming benefit biodiversity? Biological Conservation 122:113-130.

Hoobs RJ, Higgs E \& JA Harris. 2009. Novel ecosystems: implication for conservation and restoration. Trends in Ecology and Evolution 24 (11): 599-605.

Hughes AR, Byrnes JE, Kimbro DL \& Stachowicz JJ. 2007. Reciprocal relationships and potential feedbacks between biodiversity and disturbance. Ecology Letters 10: 849-864. 
Hyvonen T, Ketoja E, Salonen J, Jalli H \& Tiainen J. 2003. Weed species diversity and community composition in organic and conventional cropping of spring cereals. Agriculture, Ecosystems and Environment 97: 131-149.

IFOAM. 2007. Normas de IFOAM para la producción y el procesamiento orgánicos. Disponible en: https://www.ifoam.bio/sites/.../norms_eng_v4_20090113.pdf.

Kelemen E, Nguyen G, Gomerio T, Kovács E, Choisis JP, Choisis N, Paoletti M, Podmaniczky L, Rischawy J, Sarthou JP, Herzog F, Dennis P \& Bálaz K. 2013. Farmers' perceptions of biodiversity: lessons from a discourse-based deliberative valuation study. Land Use Policy 35: 318-328.

Krebs JR, Wilson JD, Bradbury RB, Siriwardena GM. 1999. The second silent spring? Nature 400: 611-612.

Légère A, Stevenson FC \& Benoit DL. 2005. Diversity and assembly of weed communities: contrasting responses across cropping systems. Weed Research. 45: 303-315.

Lugo AE. 1988. The future of the forest ecosystem rehabilitation in the Tropics. Environment $30(7): 17-20 \& 41-45$.

Martínez-Ghersa MA, Ghersa CM \& Satorre EH. 2000. Coevolución of agriculture systems and their weed companions: implications for research. Field Crops Research 67: 181190.

Matson PA, Parton WJ, Power AG \& Swif MJ. 1997. Agricultural intensification and ecosystem properties. Science 277: 504-509.

Marzocca A. 1979. Manual de Malezas. Ed. Hemisferio Sur, Bs As. 564 p.

McLaughlin A \& Mineau P. 1995. The impact of agricultural practices on biodiversity. Agriculture, Ecosystems \& Environment 55: 201-212.

Paleologos MF, CC Flores, SJ Sarandon, SA Stupino \& MM Bonicatto. 2008. Abundancia y diversidad de la entomofauna asociada a ambientes seminaturales en fincas horticolas de La Plata, Buenos Aires, Argentina. Revista Brasileira de Agroecología 3(1): 28-40.

Palmer MW \& Maurer T. 1997. Does diversity beget diversity?. A case study of crops and weed. Journal of Vegetation Science 8: 235-240.

Petit S, Munier-Jolain N, Bretagnolle V, Bockstaller C, Gaba S, Cordeau S \& Colbach N. 2015. Ecological intensification through pesticide reduction: weed control, weed biodiversity and sustainability in arable farming. Environmental Management 56(5): 10781090.

Poggio SL, Satorre EH \& de la Fuente EB. 2004. Structure of weed communities occurring in pea and wheat crops in the Rolling Pampa (Argentina). Agriculture, Ecosystem \& Environment 103: 225-235. 
Poggio S. 2005. Structure of weed communities occurring in monoculture and intercropping of field pea and barley. Agriculture, Ecosystems and Environment 19: 48-58.

Poggio SL, Chaneton EJ \& Ghersa CM. 2013. The arable plant diversity of intensively managed farmland: Effects of field position and crop type at local and landscape scales. Agriculture, Ecosystems \& Environment 166: 55-64.

Puricelli E, Faccini D, Nisenshon L \& Tuesca D. 2012. Weed cover, frequency and diversity in field plots and edges in the soybean central region of Argentina. Agricultural Science 3(5): 631-639.

Pyšek P \& Lepš J. 1991. Response of weed community to nitrogen fertilization: a multivariate analysis. Journal of Vegetation Science 2: 237-244.

Radosevich SR, Holt J \& Ghersa CM. 2007. Ecology of weeds and invasive plants. Relationship to agriculture and natural resources management. 3ra edition. John Wiley \& Sons: Nueva York. 454 p.

Rahmamn G. 2011. Biodiversity and Organic farming: What do we Know?. Agriculture and Forestry Research 61(3): 189-208.

Roschewitz I, Gabriel D, Tscharntke T \& Thies C. 2005. The effects of landscape complexity on arable weed arable diversity in organic and conventional farming. Journal of Applied Ecology 42 (5): 873-882.

Rundlöf M, Edlund M \& Smith HG. 2010. Organic farming at local and landscape scales benefits plant diversity. Ecography 33: 514-522.

Ryan MR, Smith RG, Mirsky SB, Mortensen DA \& Seidel R. 2010. Management filters and species traits: weed community assembly in long-term organic and conventional systems. Weed Science 58: 265-277.

Sánchez Vallduví G \& Sarandón SJ. 2014. Principios de manejo ecológico de malezas. En: Sarandón SJ \& CC Flores (Eds.). Agroecología: bases teóricas para el diseño y manejo de agroecosistemas sustentables. Colección libros de cátedra. Editorial de la Universidad Nacional de La Plata. Capítulo 11: 286-313. Disponible en http://sedici.unlp.edu.ar/handle/10915/37280.

Scursoni J, Gigón R, Martín AN, Vigna M, Leguizamón ES, Istilart C, \& López R. 2014. Changes in Weed Communities of Spring Wheat Crops of Buenos Aires Province of Argentina. Weed Science 62(1): 51-62.

Stilma ESC, Smit AB, Geerling-Eiff FA, Struik PC, Vosman B \& Korevaar H. 2009. Perception of biodiversity in arable production systems in the Netherlands. NJASWageningen Journal of Life Science 56: 391-404.

Stupino SA, Ferreira AC, Frangi JL \& Sarandón SJ. 2007. Agrobiodiversidad vegetal en sistemas hortícolas orgánicos y convencionales (La Plata, Buenos Aires, Argentina). Revista Brasileira de Agroecología, Asociación Brasilera de Agroecología. Edición 
especial: Resúmenes del II Congreso Brasileiro de Agroecología, 22 al 25 de Noviembre de 2004. Vol 2 (1): 339-342.

Stupino SA, lermanó MJ, Gargoloff NA \& Bonicatto MM. 2014. La biodiversidad en los agroecosistemas. En: Sarandón J \& Flores C (Eds.). Agroecología: Bases teóricas para el diseño y manejo de agroecosistemas sustentables. Colección de libros de cátedra, Secretaria de Asuntos Académicos-Edulp, UNLP.

Suárez SA, de la Fuente EB, Ghersa CM \& León RJC. 2001. Weed community as an indicator of summer crop yield and site quality. Agronomy Journal 93: 524-530.

Travlos IS, Cheimona N, Roussis I \& Bilalis DJ. 2018. Tillage and fertilization effect on weeds. Frontiers in Environmental Science 6(11): 1-10.

Tscharntke T, Clough Y, Wanger T, Jackson L, Motzke I, Perfecto I, Vandermeer J \& Whitbread A. 2012. Global food security, biodiversity conservation and the future of agricultural intensification. Biological Conservation 151(1): 53-59.

Tang L, Wan K, Chuanpeng C \& Chen F. 2013. Effect of fertilization patterns on the assemblage of weed communities in an upland winter wheat field. Journal of Plant Ecology 7(1): 39-50.

Tuck SL, Winqvist C, Mota F, Ahnström J, Turnbull LA \& Bengtsson J. 2014. Land-use intensity and the effects of organic farming on biodiversity: a hierarchical meta-analysis. Journal of Applied Ecology 51: 746-755.

Urban S. 2010. Synanthropic vegetation: pattern of various disturbances on life history traits. Acta Botanica Croata 69(2): 215-227.

van Elsen T. 2000. Species diversity as a task for organic agriculture in Europe. Agriculture, Ecosystems and Environment 77: 101-109.

Zanin G, Otto S, Riello L \& Borin M. 1997. Ecological interpretation of weed flora dynamics under different tillage systems. Agriculture, Ecosystems and Environment 66: 177-188. 